\title{
Aerobic Visible Light-Driven Borylation of Heteroarenes in a Gel Nanoreactor
}

Jorge C. Herrera-Luna, ${ }^{a}$ David Díaz Díaz, ${ }^{\text {b,c,d }}$ Alex Abramov, ${ }^{d}$ Susana Encinas, ${ }^{a}$ M. Consuelo Jiménez, ${ }^{\star a}$ and Raúl Pérez-Ruiz ${ }^{\star a}$

a Departamento de Química, Universitat Politècnica de València (UPV), Camino de Vera S/N, 46022, Valencia, Spain.

b Departamento de Química Orgánica, Universidad de La Laguna, Avda. Astrofísico Francisco Sánchez 3, 38206, La Laguna, Spain.

c Instituto de Bio-Orgánica Antonio González, Universidad de La Laguna, Avda. Astrofísico Francisco Sánchez 3, 38206, La Laguna, Spain.

d Institut für Organische Chemie, Universität Regensburg, Universitätsstr. 31, 93053, Regensburg, Germany.

\section{Table of contents}

* Materials and methods

* General procedure

* Synthesis of LMW Gelators

* Searching the optimal conditions

* Kinetic studies

* Frozen model reaction procedure

* Field-emission scanning electron microscopy (FESEM)

* Determination of gel-to-sol transition temperature $\left(T_{\text {gel }}\right)$

* UV-vis absorption spectra

* Singlet oxygen

* GCMS byproduct

* Trapping reaction

* Sunlight irradiation and $1 \mathrm{mmol}$ scale

* GC Chromatograms

* Characterization of compounds 


\section{Materials and methods}

All reagents ( $\geq 97 \%$ purity) and solvents ( $\geq 99 \%$ purity) were purchased from commercial suppliers (Merck, TCI, Apollo Scientific, Fluorochem, Scharlab) and used as received unless otherwise indicated. Reactions were carried out in Metria®-Crimp Headspace clear vial flat bottom (10 mL, $\varnothing 20 \mathrm{~mm}$ ) sealed with Metriaß-aluminium crimp cap with moulded septum butyl/natural PTFE $(\varnothing 20 \mathrm{~mm})$. Irradiation was performed with a cool white LED (LED Cree MK-R, cold-white, $11.6 \mathrm{~V}, 700 \mathrm{~mA}, \mathrm{P}=8.5 \mathrm{~W}$ ). TLC was performed on commercial $\mathrm{SiO}_{2}$-coated aluminium and plastic sheets (DC60 F254, Merck). Visualization was done by UV-light $(254 \mathrm{~nm})$. Product were isolated materials after TLC on silica gel (Merck, mesh 35-70, $60 \AA$ pore size) and their corresponding yields were determined by quantitative GC-FID measurements on an Agilent 8860 GC-System with $\mathrm{N}_{2}$ as carrier gas. Dodecanenitrile was used as an internal standard in the GC-FID quantitative measurements; yield products were estimated as: [conversion $x$ selectivity]/mass balance. Determination of purity and structure confirmation of the literature known products was performed by ${ }^{1} \mathrm{H}$ NMR, ${ }^{13} \mathrm{C}$ NMR and high-resolution mass spectrometry (HRMS) in case of unknown products. NMR spectral data were collected on a Bruker Advance $400\left(400 \mathrm{MHz}\right.$ for ${ }^{1} \mathrm{H}$ and $101 \mathrm{MHz}$ for $\left.{ }^{13} \mathrm{C}\right)$ spectrometer at $20{ }^{\circ} \mathrm{C}$. Chemical shifts are reported in $\delta / p p m$, coupling constants $\mathrm{J}$ are given in Hertz. Solvent residual peaks were used as internal standard for all NMR measurements. The quantification of ${ }^{1} \mathrm{H}$ cores was obtained from integrations of appropriate resonance signals. Abbreviations used in NMR spectra: $s$ - singlet, $d$ - doublet, $t$ - triplet, $q-$ quartet, $\mathrm{m}$ - multiplet, bs - broad singlet, dd - doublet of doublet, ddd - doublet of doublet of doublet. HRMS was carried out was performed in the mass facility of SCSIE University of Valencia. LRMS was carried out on an HP 6890 Series GC System with Agilent 5973 Network Mass Selective Detector and $\mathrm{H}_{2}$ as carrier gas. 


\section{General procedure}

A vial $(10 \mathrm{~mL})$ was charged with the hetereoarene derivative $(20 \mu \mathrm{mol}, 1.0$ eq. $)$, diborylates (200 $\mu \mathrm{mol}, 10.0$ eq.) and the correspondent gelator ( $\mathrm{G} 1,10 \mathrm{mg} / \mathrm{mL}$ ). Acetonitrile/ $\mathrm{H}_{2} \mathrm{O}(9 / 1 \mathrm{v} / \mathrm{v})$ mixture $(1.0 \mathrm{~mL})$ was poured. Then, DIPEA $(24 \mu \mathrm{mol}, 1.2 \mathrm{eq}$.) and dodecanenitrile (20 $\mu \mathrm{mol}, 1.0 \mathrm{eq}$.) were added with $10 \mu \mathrm{L}$ Hamilton syringe. Quickly, the vial was sealed with a septum. It was heated to $150{ }^{\circ} \mathrm{C}$ for 1.5 minutes with manual stirring until complete clear solution. The vial cooled to room temperature until gel formation was observed. The reaction was irradiated with an external LED through the plain bottom side of the vial at $23^{\circ} \mathrm{C}$ during the corresponding time. Then, brine $(2 \mathrm{~mL})$ was added, and the aqueous phase was extracted with dichloromethane $(1 \mathrm{~mL})$. The reaction was monitored by GC-FID analysis. The organic phase was dried over anhydrous sodium sulfate, filtered from the drying agent, and concentrated in vacuo. After recrystallization of the excess of starting diboron derivative, which is recovered, the crude was purified via TLC plastic sheet using a hexane/ethyl acetate mixture as the mobile phase. Note: The gelator can be easily separated by filtration and reused in subsequent experiments without any detriment of its gelation properties.
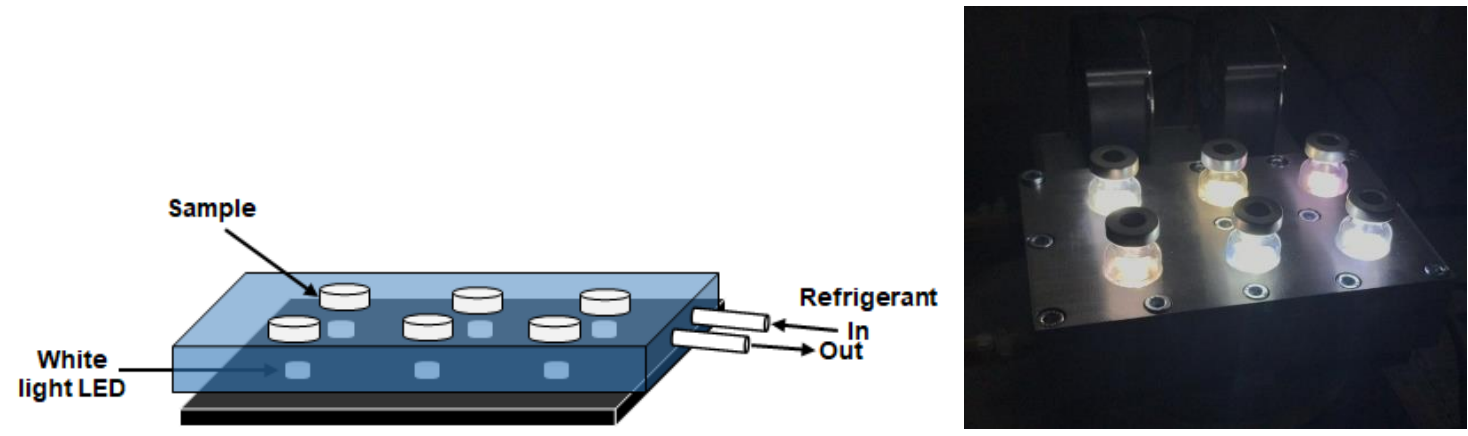

Irradiation setup

Lamp emission: cold white light LED

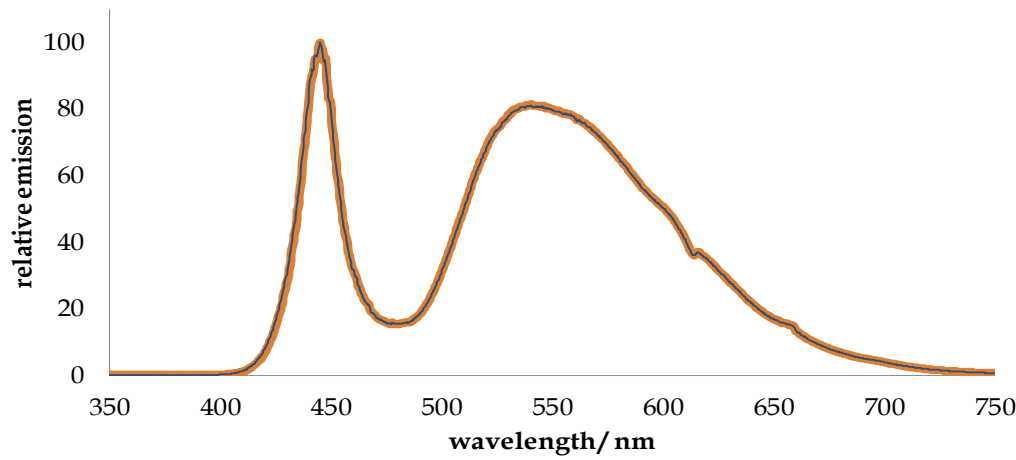




\section{Synthesis of Low Molecular Weight Gelators (LMW Gelators)}

N,N'-Bis(octadecyl)-L-Boc-glutamic Diamide (G1).

Boc-glutamic acid ( $0.01 \mathrm{~mol}, 1.0$ equiv) and octadecylamine $(0.02 \mathrm{~mol}, 2.0$ equiv) in dichloromethane $(200 \mathrm{~mL})$ were mixed. Then, 1-ethyl-3-(3(dimethylamino)propyl)carbodiimide hydrochloride (EDC $\cdot \mathrm{HCl})(0.022 \mathrm{~mol}, 2.2$ equiv) was added to the mixture and stirred at RT for $72 \mathrm{~h}$. The obtained white solid was isolated by filtration and washed three times with dichloromethane. The crude product was dissolved in THF and precipitated by water. A fine white solid was obtained (80\%). Following the procedure reported previously Soft Matter 2007, 3, 1312-1317.

${ }^{1} \mathrm{H}$ NMR $\left(300 \mathrm{MHz} \mathrm{CDCl}_{3}\right) \delta 6.69(\mathrm{br}, 1 \mathrm{H}), 6.32(\mathrm{br}, 1 \mathrm{H}), 5.77(\mathrm{br}, 1 \mathrm{H}), 4.08(\mathrm{br}, 1 \mathrm{H})$, 3.26-3.22 (m, 4H), 2.41-2.27 (m, 2H), 2.06-1.93 (m, 2H), 1.58-1.46 (m, 4H), $1.43(\mathrm{~s}$, $9 \mathrm{H}), 1.25(\mathrm{~s}, 60 \mathrm{H}), 0.87(\mathrm{t}, 6 \mathrm{H}) \mathrm{ppm}$.

(S,S)-Dodecyl-3-[2(3-dodecyl-ureido)cyclohexyl]urea (G2).

A solution of dodecylisocyanate $(15 \mathrm{mmol}, 2.0$ equiv) in toluene $(20 \mathrm{~mL})$ was slowly added to a solution of $(S, S)$-1,2-cyclohexyldiamine (7 mmol, 1.0 equiv) in toluene (100 $\mathrm{mL}$ ). The reaction mixture was stirred for $16 \mathrm{~h}$ at $\mathrm{RT}$ and $2 \mathrm{~h}$ at $100^{\circ} \mathrm{C}$. After cooling to $\mathrm{RT}$, the gel-like reaction mixture was filtered to give a white waxy solid. The waxy solid was further stirred for $16 \mathrm{~h}$ with dichloromethane $(50 \mathrm{~mL})$ and collected by filtration. This procedure was repeated with diethyl ether. After drying, a white solid was obtained (70\%). Following the procedure reported previously Chem. - Eur. J. 1999, 5, 937-950

${ }^{1} \mathrm{H}$ NMR $\left(300 \mathrm{MHz}, \mathrm{CDCl}_{3}\right) \delta 5.18(\mathrm{br}, 2 \mathrm{H}), 4.67(\mathrm{br}, 2 \mathrm{H}), 3.42(\mathrm{~m}, 2 \mathrm{H}), 3.08(\mathrm{~m}, 4 \mathrm{H})$, $2.03(\mathrm{~d}, 2 \mathrm{H}), 1.72(\mathrm{~m}, 6 \mathrm{H}), 1.45(\mathrm{~m}, 4 \mathrm{H}), 1.25(\mathrm{~s}, 36 \mathrm{H}), 0.87(\mathrm{t}, 6 \mathrm{H}) \mathrm{ppm}$. 
Searching the optimal conditions

Table S1.

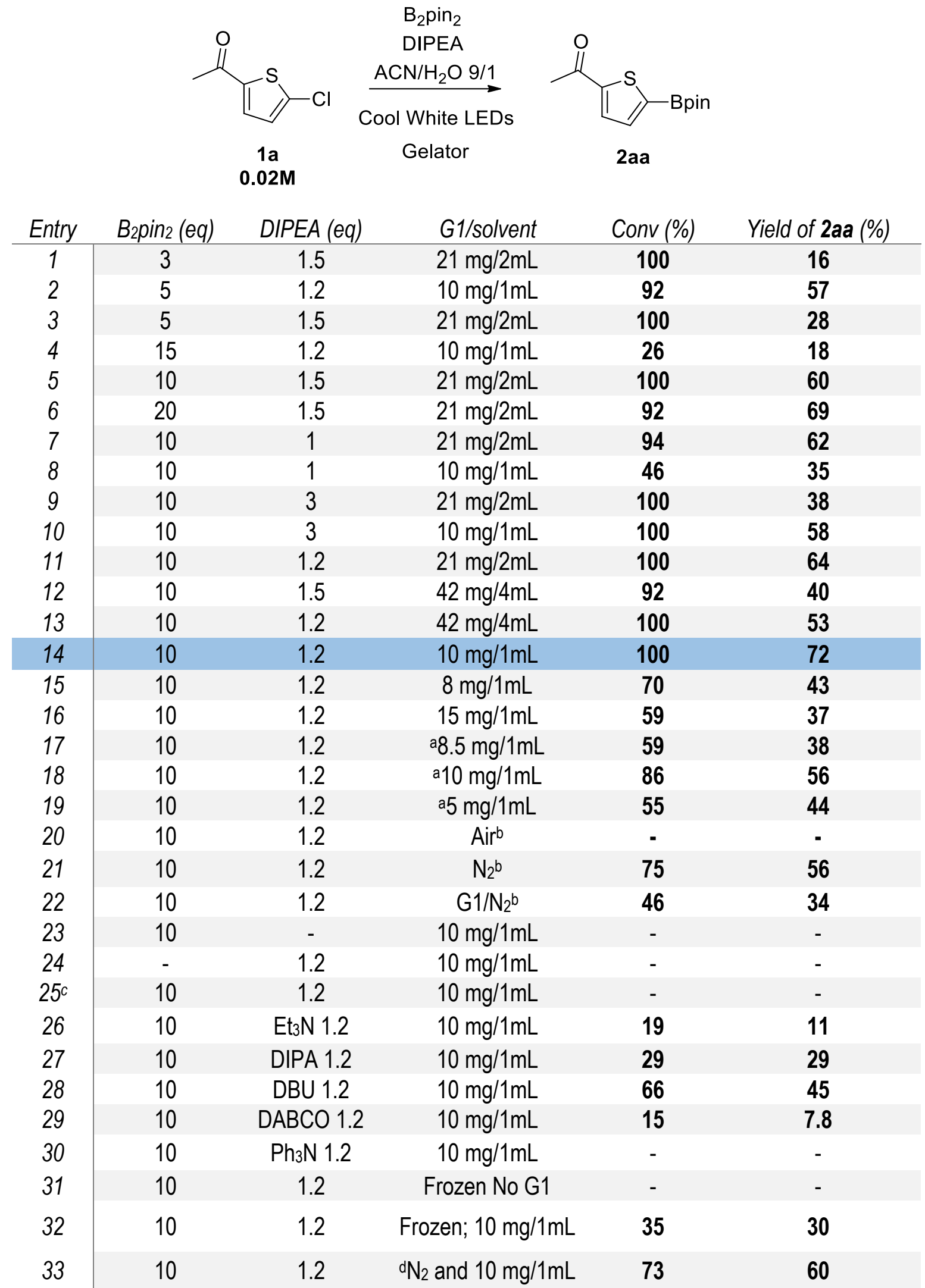

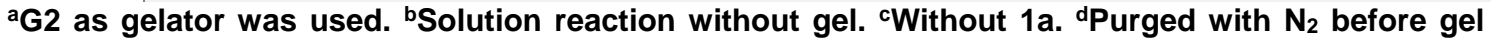
formation. 


\section{Kinetic studies}<smiles>CC(=O)c1ccc(Cl)s1</smiles>

$1 \mathrm{a}$

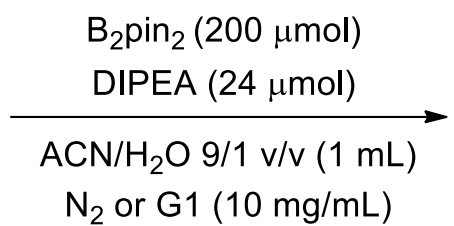

Cool White LEDs<smiles>CC(=O)c1ccc(Br)s1</smiles>

2aa

$20 \mu \mathrm{mol}$

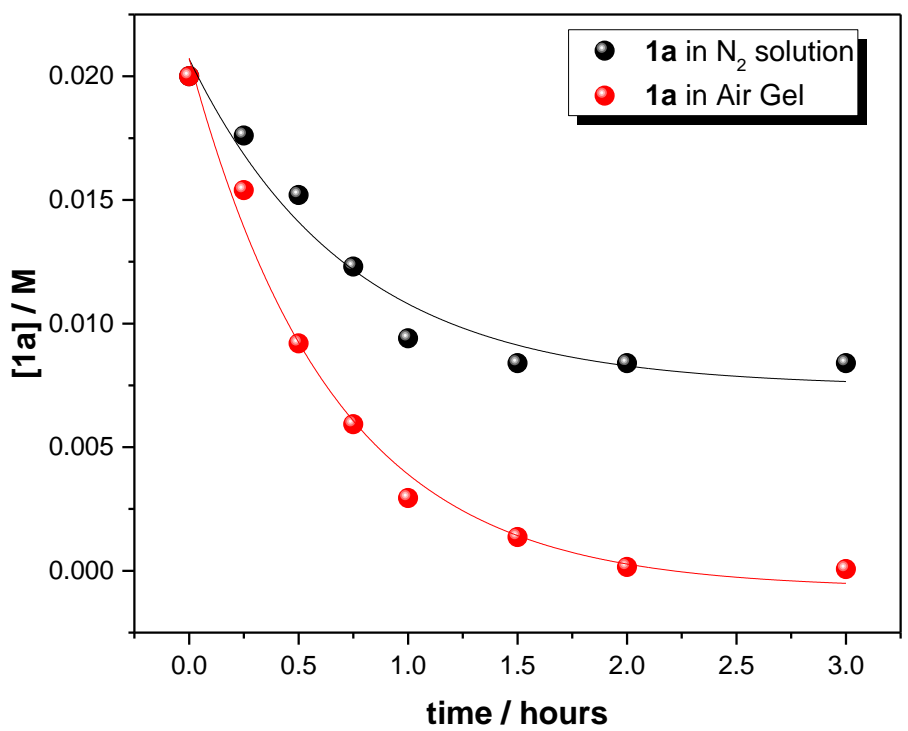

Figure S1. Variation of 1a concentration upon irradiation time in different conditions.

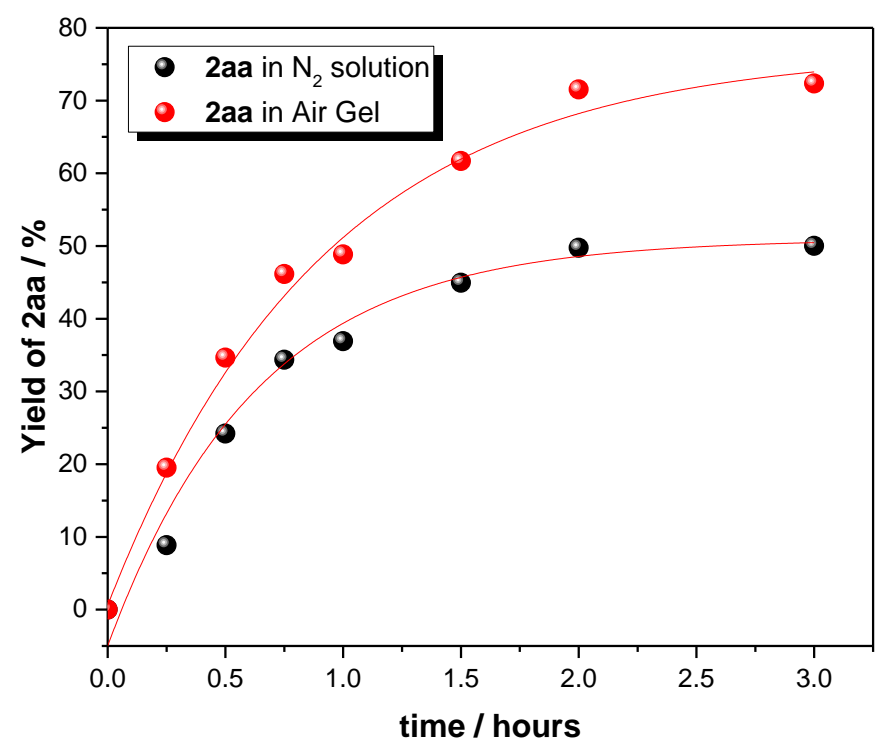

Figure S2. Formation of 2aa upon irradiation time in different conditions. 


\section{Frozen model reaction procedure}

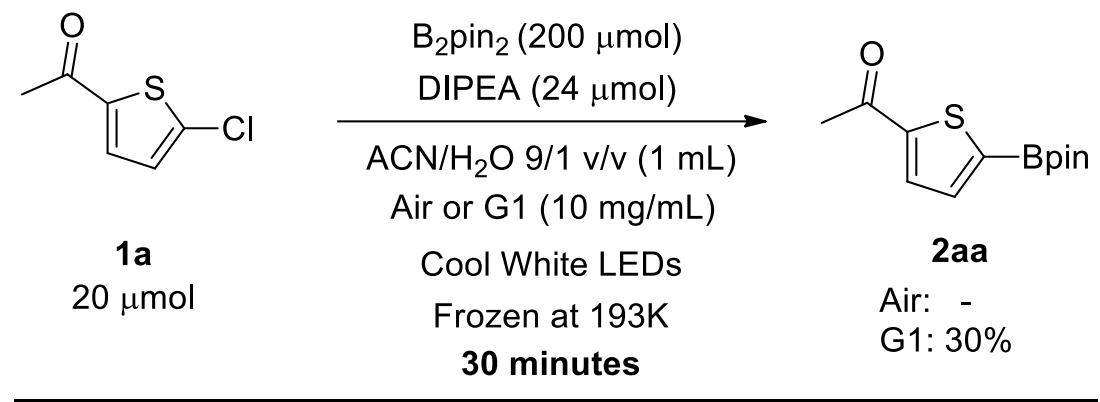

A vial $(10 \mathrm{~mL})$ was charged with 1-(5-chlorothiophen-2-yl)ethenone $(3.2 \mathrm{mg}, 20 \mu \mathrm{mol}$, 1.0 equiv.), bispinacolato diboron ( $50 \mathrm{mg}, 200 \mu \mathrm{mol}, 10.0$ equiv.) and the correspondent gelator $(\mathrm{G} 1,10 \mathrm{mg} / \mathrm{mL})$. Acetonitrile $/ \mathrm{H}_{2} \mathrm{O}(9 / 1 \mathrm{v} / \mathrm{v})$ mixture $(1.0 \mathrm{~mL})$ was poured, and it was stirred until almost complete solution. Then, DIPEA $(4.2 \mu \mathrm{L}, 24 \mu \mathrm{mol}, 1.2$ equiv.) and dodecanenitrile $(4.4 \mu \mathrm{L}, 20 \mu \mathrm{mol}, 1.0$ equiv.) were added with $10 \mu \mathrm{L}$ Hamilton syringe. Quickly, the vial was sealed with a septum. It was heated to $150^{\circ} \mathrm{C}$ with heatgun for 1.5 minutes with manual stirring until complete clear solution. The vial cooled to room temperature until gel formation was observed. The vial was frozen at $193 \mathrm{~K}$ for 2 hours. Then, the reaction was irradiated with an external LED through the plain bottom side of the vial at $23{ }^{\circ} \mathrm{C}$ for 30 minutes. The reaction was monitored by GC-FID analysis. The gel was broken with dichloromethane $(2 \mathrm{~mL})$ and it was clean with brine $(6 \mathrm{~mL})$. The organic phase was dried over anhydrous sodium sulfate, filtered from the drying agent, and concentrated in vacuo. 


\section{Field-emission scanning electron microscopy (FESEM)}

The equipment in operation in the UPV Microscopy Service is the ZEISS ULTRA 55 model, incorporating the following detectors:

-A Secondary Electron Detector (SE2), which provides an SEM topography image of the sample surface with a large depth of field.

- A Secondary Electron In-Lens Detector located inside the electron column, which works with low energy secondary electrons and provides images with a higher resolution.

- A Backscattered Electron Detector (AsB) which is sensitive to the variation of atomic number in the elements present in the sample; therefore, it is used to observe changes in the chemical composition of the specimen.

- A Backscattered Electron In-lens Detector (EsB), independent of the secondary In-lens detector, which provides a pure backscattered signal with no secondary electron contamination and very low acceleration potential.

- An X-Ray Dispersive Energy Detector, EDS, (Oxford Instruments) which receives Xrays from each surface point the electron beam passes over. 


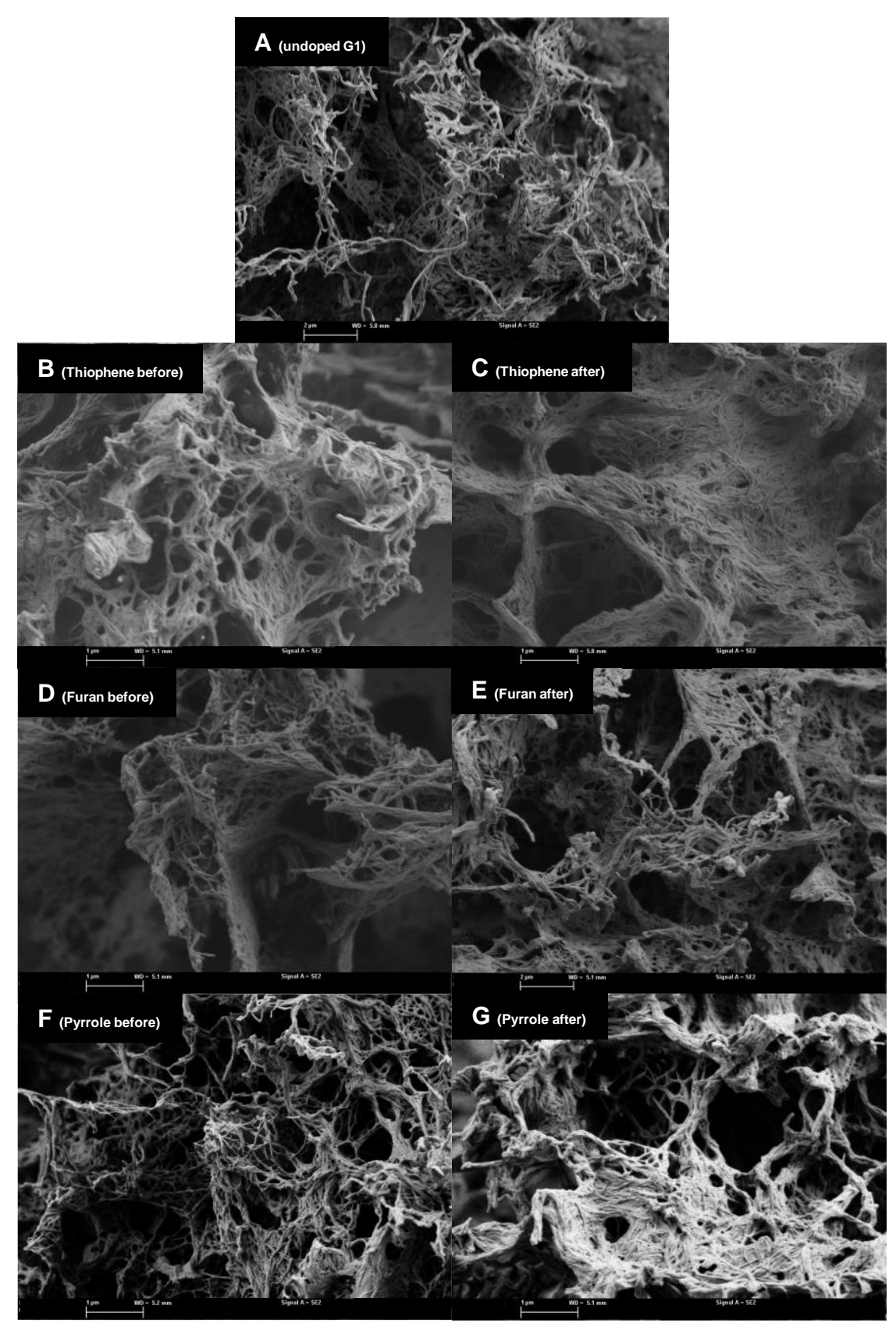

Figure S3. Representative field-emission scanning electron microscopy (FESEM) images. A: undoped gel of $\mathbf{G 1}(10 \mathrm{mg} / \mathrm{mL})$. B, C: gel of $\mathbf{G 1}(10 \mathrm{mg} / \mathrm{mL})$ doped with 2acetyl-5-chlorothiophene (3.2 mg), $B_{2}$ pin $_{2}(50 \mathrm{mg})$ and DIPEA (3.1 mg). D, E: gel of G1 (10 mg/mL) doped with 2-acetyl-5-bromofuran (3.8 mg), B pin $_{2}(50 \mathrm{mg})$ and DIPEA (3.1 $\mathrm{mg})$. F, G: gel of G1 (10 mg/mL) doped with 2-acetyl-5-bromo-1H-methylpyrrole (4.0 mg), $\mathrm{B}_{2} \mathrm{pin}_{2}(50 \mathrm{mg})$ and DIPEA (3.1 mg). 


\section{Determination of gel-to-sol transition temperature ( $\left.T_{\text {gel }}\right)$}

$T_{\text {gel }}$ values were determined using a custom-made set-up where a sealed vial was placed into a mold of an alumina block and heated up at $1^{\circ} \mathrm{C} / 5 \mathrm{~min}$ using an electric heating plate equipped with a temperature control couple as previously described (see for instance, Chem. Commun. 2015, 51, 16848-16851). The temperature at which the gel started to break was defined as $T_{\text {gel }}$ with an estimated error of $\pm 2{ }^{\circ} \mathrm{C}$ after several heating-cooling cycles. 


\section{UV-vis absorption spectra}
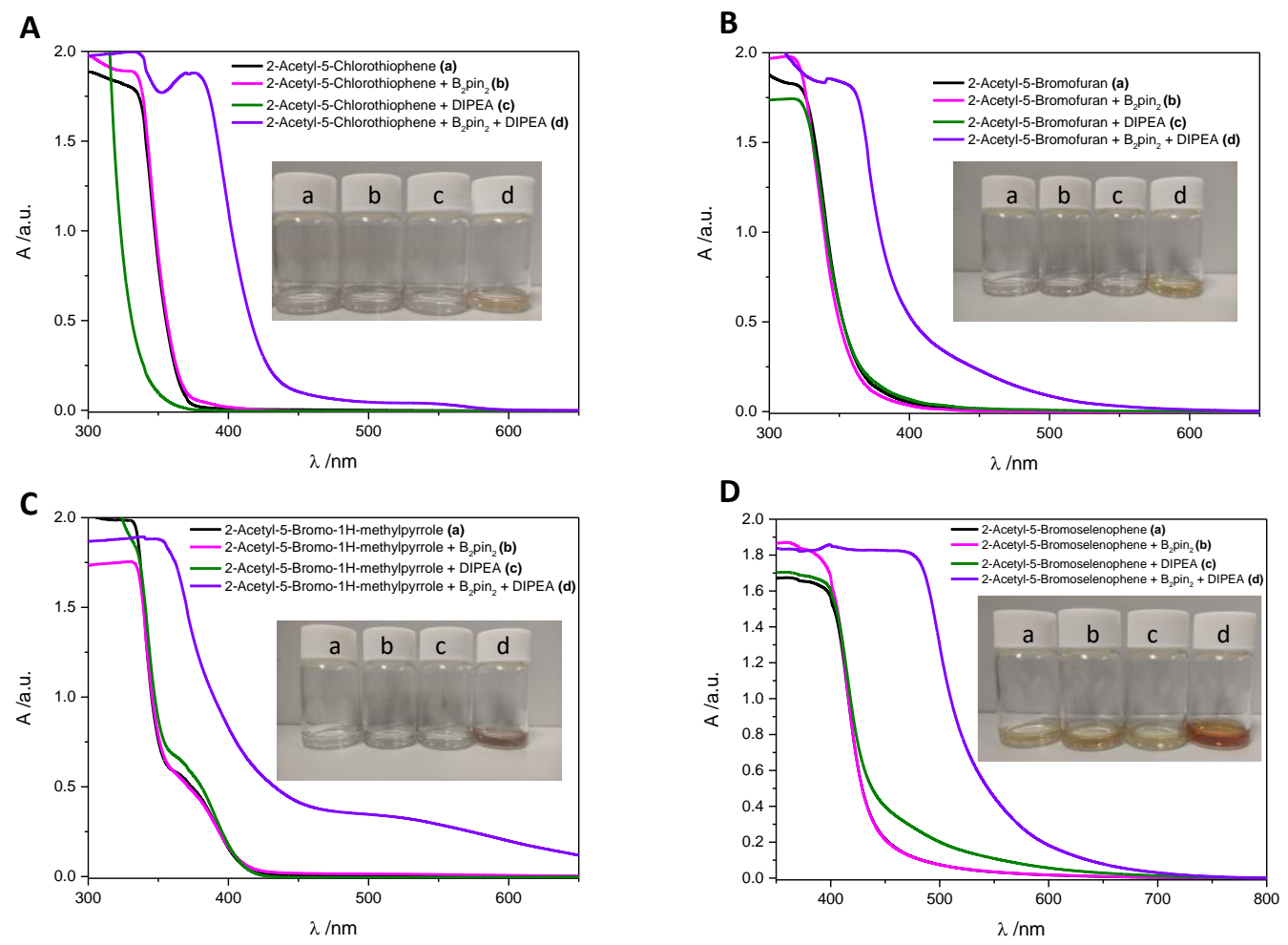

Figure S4. UV-vis absorption spectra. Inset: photographs of different solutions. A: 2Acetyl-5-Chlorothiophene $(0.02 \mathrm{mmol}), B_{2} p_{2}(0.2 \mathrm{mmol})$ and DIPEA $(0.024 \mathrm{mmol})$ in ACN/ $\mathrm{H}_{2} \mathrm{O} 9 / 1 \mathrm{v} / \mathrm{v} 1 \mathrm{~mL}$. B: 2-Acetyl-5-Bromofuran (0.02 mmol), $\mathrm{B}_{2} \mathrm{pin}_{2}(0.2 \mathrm{mmol})$ and DIPEA (0.024 mmol) in ACN/ $\mathrm{H}_{2} \mathrm{O}$ 9/1 v/v $1 \mathrm{~mL}$. C: 2-Acetyl-5-Bromo-1H-methylpyrrole (0.02 mmol), $B_{2} p_{2}$ ( $\left.0.2 \mathrm{mmol}\right)$ and DIPEA (0.024 mmol) in ACN/H ${ }_{2} \mathrm{O} 9 / 1 \mathrm{v} / \mathrm{v} 1 \mathrm{~mL}$. D: 2-Acetyl-5-Bromoselenophene (0.02 mmol), $B_{2} p_{2}(0.2 \mathrm{mmol})$ and DIPEA (0.024 mmol) in $\mathrm{ACN} / \mathrm{H}_{2} \mathrm{O}$ 9/1 v/v $1 \mathrm{~mL}$. 


\section{Singlet Oxygen}

Time-resolved measurements of ${ }^{1} \mathrm{O}_{2}$ luminiscence were performed in a front face setup in FLS 1000 (Edinburgh Instruments) spectrophotometer by making use of a 450W Xe lamp and a NIR sensitive PMT 1700 detector (H10330C-75, Hamamatsu) in Peltier Cooled Housing.

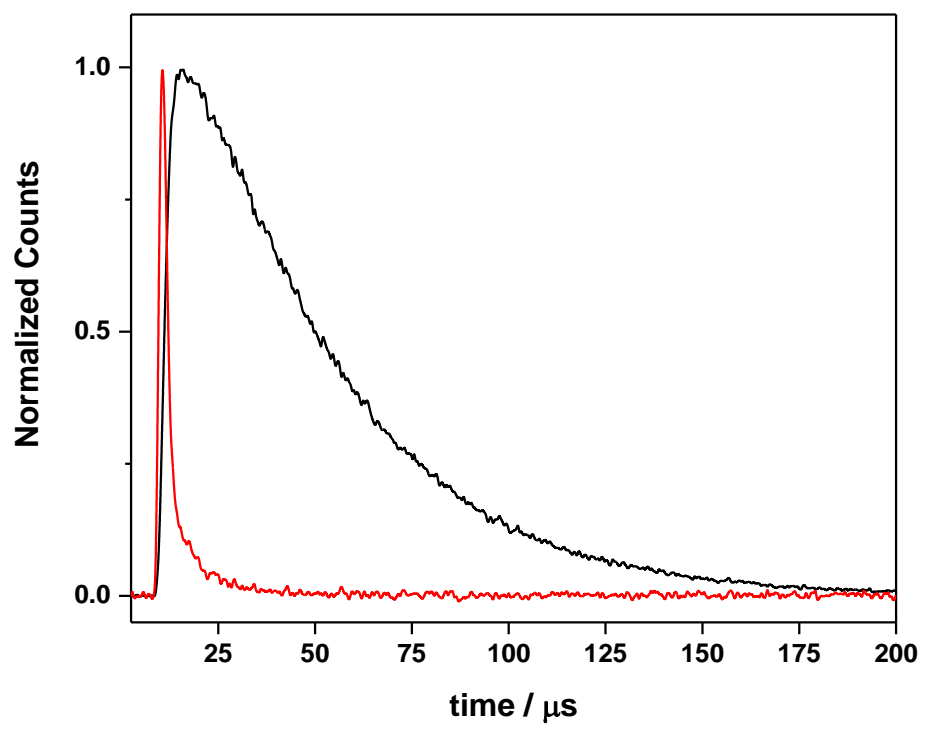

Figure S5. Decay traces of the ${ }^{1} \mathrm{O}_{2}$ emission $(1275 \mathrm{~nm})$ generated by an aerobic $\mathrm{ACN} / \mathrm{H}_{2} \mathrm{O}(9 / 1 \mathrm{v} / \mathrm{v})$ solution of; black: Rose Bengal $\left(0.1 \mathrm{mM}, \lambda_{\mathrm{exc}}=532 \mathrm{~nm}\right)$; red: $1 \mathrm{a}+\mathrm{B}_{2} \mathrm{pin}_{2}+$ DIPEA $\left(20 \mathrm{mM}, 200 \mathrm{mM}\right.$ and $24 \mathrm{mM}$, respectively, $\left.\lambda_{\mathrm{exc}}=400 \mathrm{~nm}\right)$. 


\section{GC-MS Byproduct}

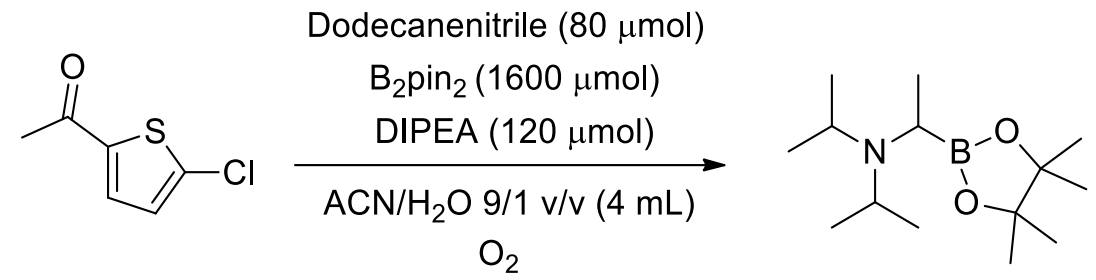

$1 a$

Cool White LEDs

$80 \mu \mathrm{mol}$ 3 hours

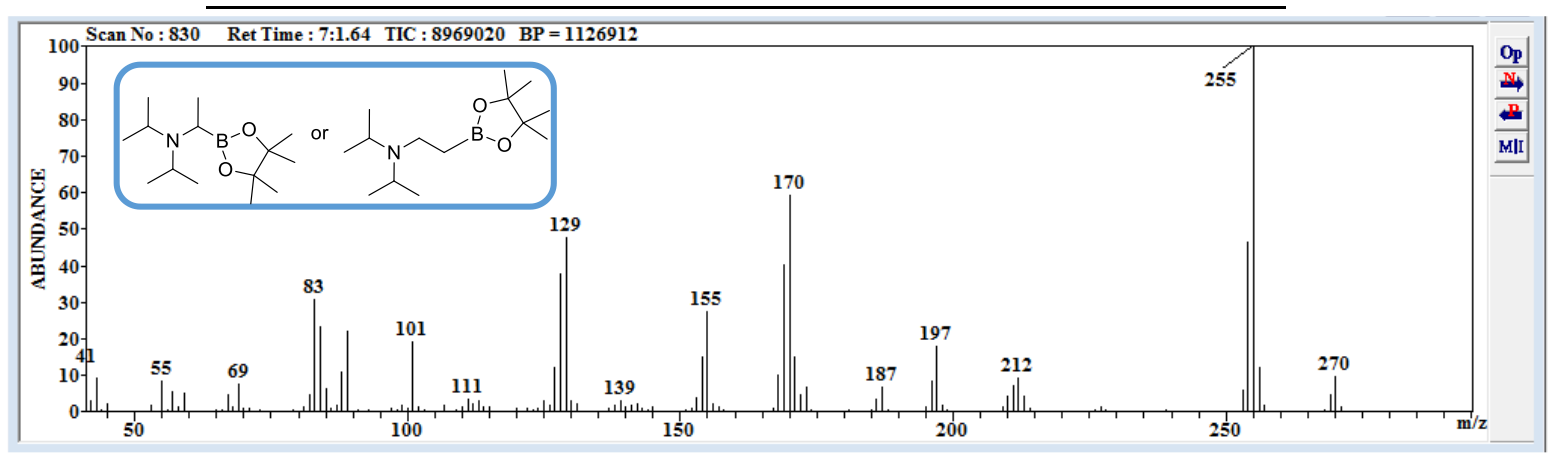




\section{Trapping reaction}

A vial (10 mL) was charged with heteroarene derivative (20 $\mu$ mol, 1.0 equiv.), diborylates

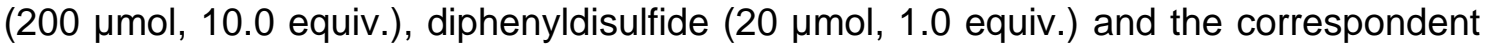
gelator $(\mathrm{G} 1,10 \mathrm{mg} / \mathrm{mL})$. Acetonitrile/ $\mathrm{H}_{2} \mathrm{O}(9 / 1 \mathrm{v} / \mathrm{v})$ mixture $(1.0 \mathrm{~mL})$ was poured, and it was stirred until almost complete solution. Then, DIPEA (24 $\mu \mathrm{mol}, 1.2$ equiv.) and dodecanenitrile ( $20 \mu \mathrm{mol}, 1.0$ equiv.) were added with $10 \mu \mathrm{L}$ Hamilton syringe. Quickly, the vial was sealed with a septum. It was heated to $150^{\circ} \mathrm{C}$ for 1.5 minutes with manual stirring until complete clear solution. The vial cooled to room temperature until gel formation was observed. The reaction was irradiated with an external LED through the plain bottom side of the vial at $23^{\circ} \mathrm{C}$ for 3 hours. Then, brine $(6 \mathrm{~mL})$ was added, and the aqueous phase was extracted with dichloromethane $(2 \mathrm{~mL})$. The reaction was monitored by GC-FID analysis. The organic phase was dried over anhydrous sodium sulfate, filtered from the drying agent, and concentrated in vacuo. After crystallization of the excess of starting diboron derivative, the crude was purified via TLC plastic sheet using a hexane/ethyl acetate mixture as the mobile phase.
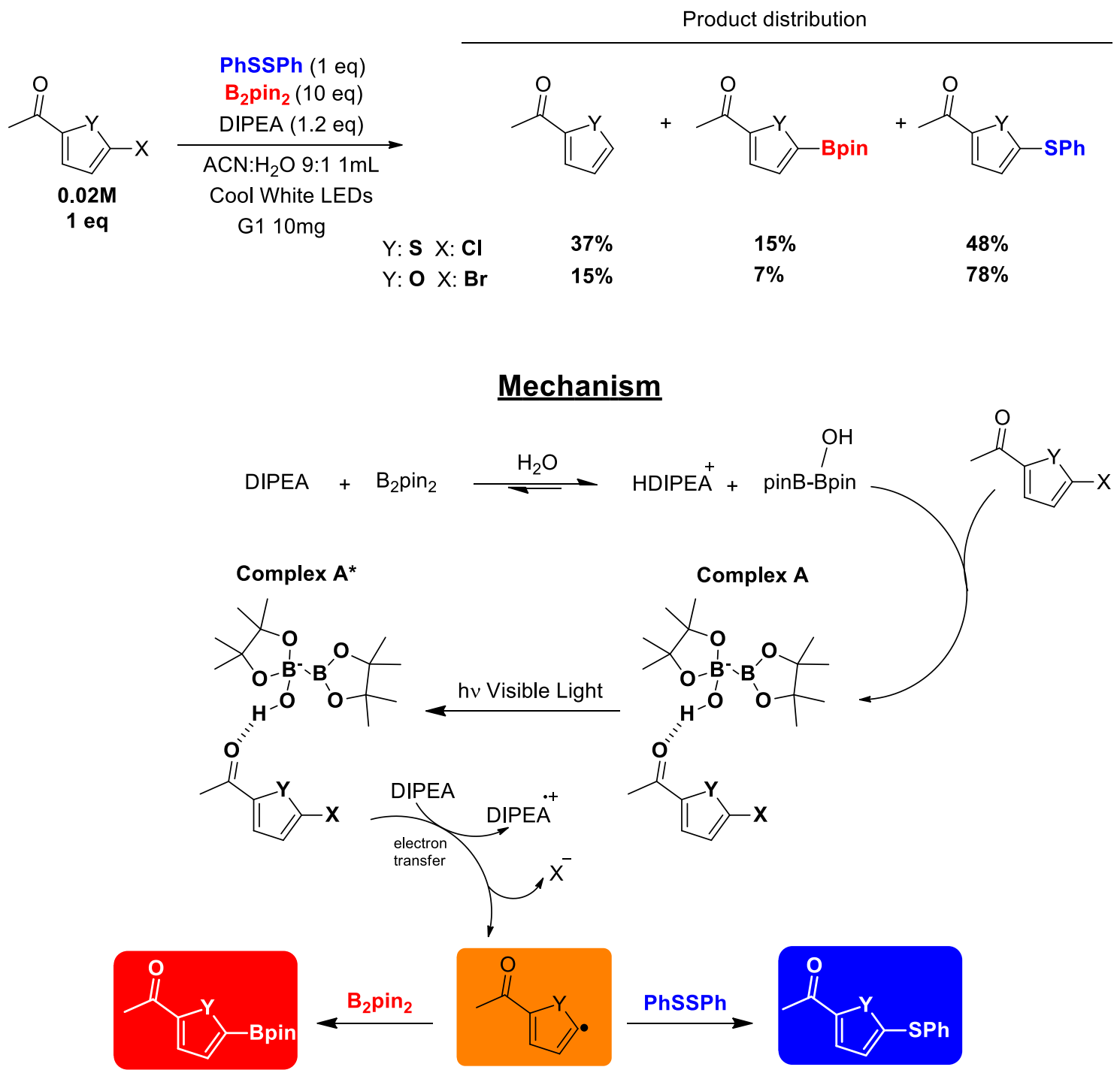


\section{Sunlight irradiation and $1 \mathrm{mmol}$ scale}

Sunlight irradiation

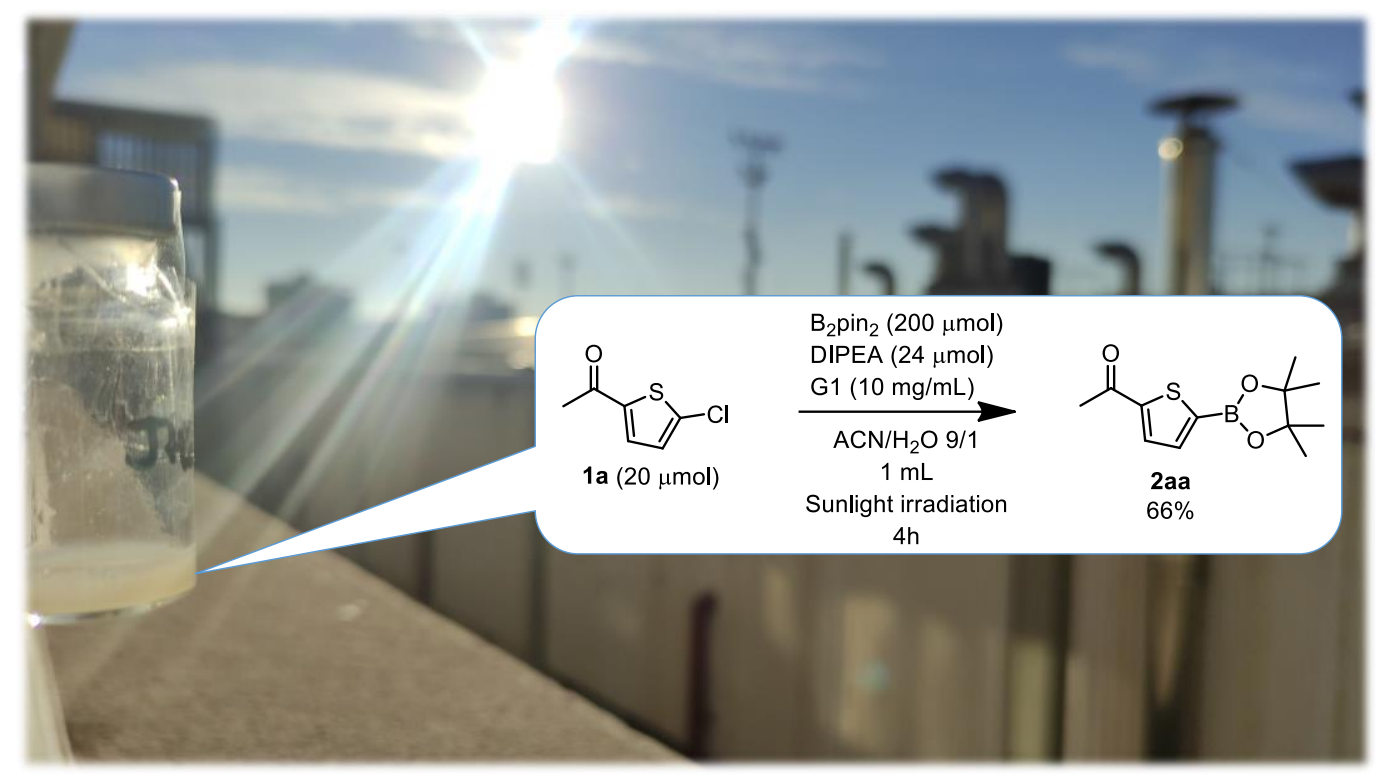

The compound 2aa (CAS 942070-32-8) was prepared according to the general procedure using 1-(5-chlorothiophen-2-yl)ethenone (3.2 mg, $20 \mu \mathrm{mol}, 1.0$ equiv.), bis(pinacolato)diboron (50.6 mg, $200 \mu \mathrm{mol}, 10.0$ equiv.), dodecanenitrile ( $4.4 \mu \mathrm{L}, 20$ $\mu \mathrm{mol}, 1.0$ equiv.) as internal standard and DIPEA (4.2 $\mu \mathrm{L}, 24 \mu \mathrm{mol}, 1.2$ equiv.) and G1 $(10 \mathrm{~g} / \mathrm{L})$. The reaction mixture was irradiated with sunlight for 4 hours, obtaining $66 \%$ product yield according to GC-FID analysis.

Location: Camí de Vera S/N, Chemistry Department, Universitat Politècnica de València (UPV), Valencia, Spain (coordinate: $39.482917,-0.341642$ ), temperature: $12-16^{\circ} \mathrm{C}$, from 9:00 to 13:00. Date: 23/12/2020.

$1 \mathrm{mmol}$ scale<smiles>CC(=O)c1ccc(Cl)s1</smiles>

(162 $\mathrm{mg}, 1 \mathrm{mmol})$

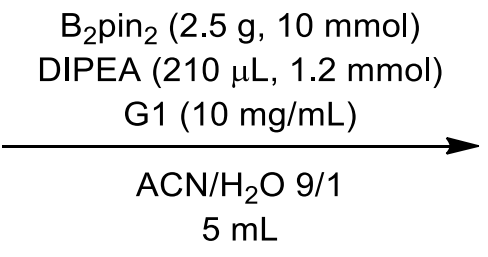

Cold White LEDs

$22 \mathrm{~h}$

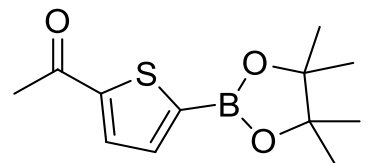

2aa

$63 \%$

The compound 2aa (CAS 942070-32-8) was prepared according to the general procedure using 1-(5-chlorothiophen-2-yl)ethenone (162 mg, $1 \mathrm{mmol}, 1.0$ eq.), bis(pinacolato)diboron ( $2.5 \mathrm{~g}, 10 \mathrm{mmol}, 10.0 \mathrm{eq}$.), dodecanenitrile $(220 \mu \mathrm{L}, 1 \mathrm{mmol}, 1.0$ equiv.) as internal standard and DIPEA ( $210 \mu \mathrm{L}, 1.2 \mathrm{mmol}, 1.2$ eq.) and G1 (10 mg/mL) in $5 \mathrm{~mL}$. The reaction mixture was irradiated for 22 hours, obtaining $63 \%$ product yield according to GC-FID analysis. 


\section{GC chromatograms}

Thiophenes
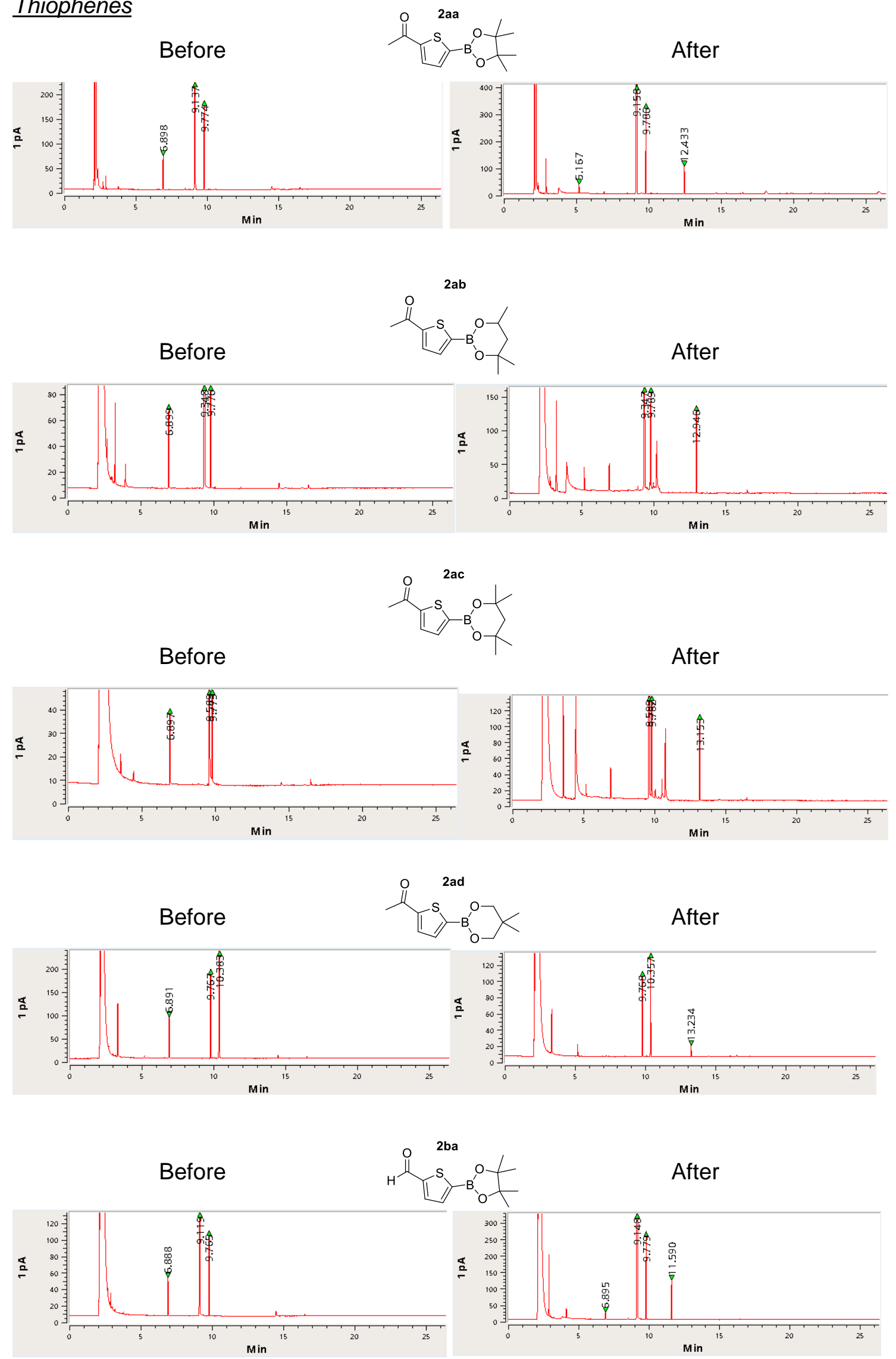

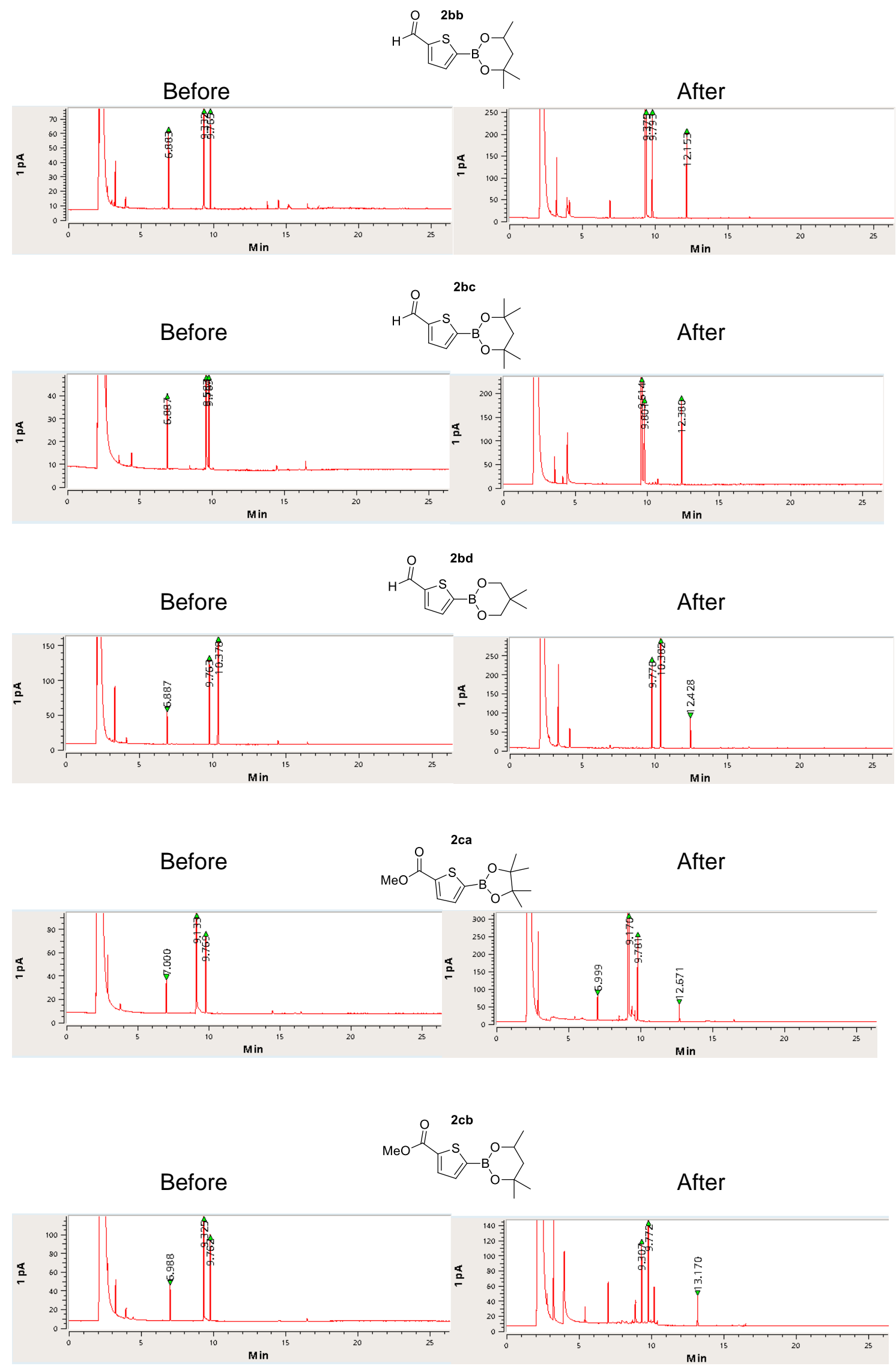

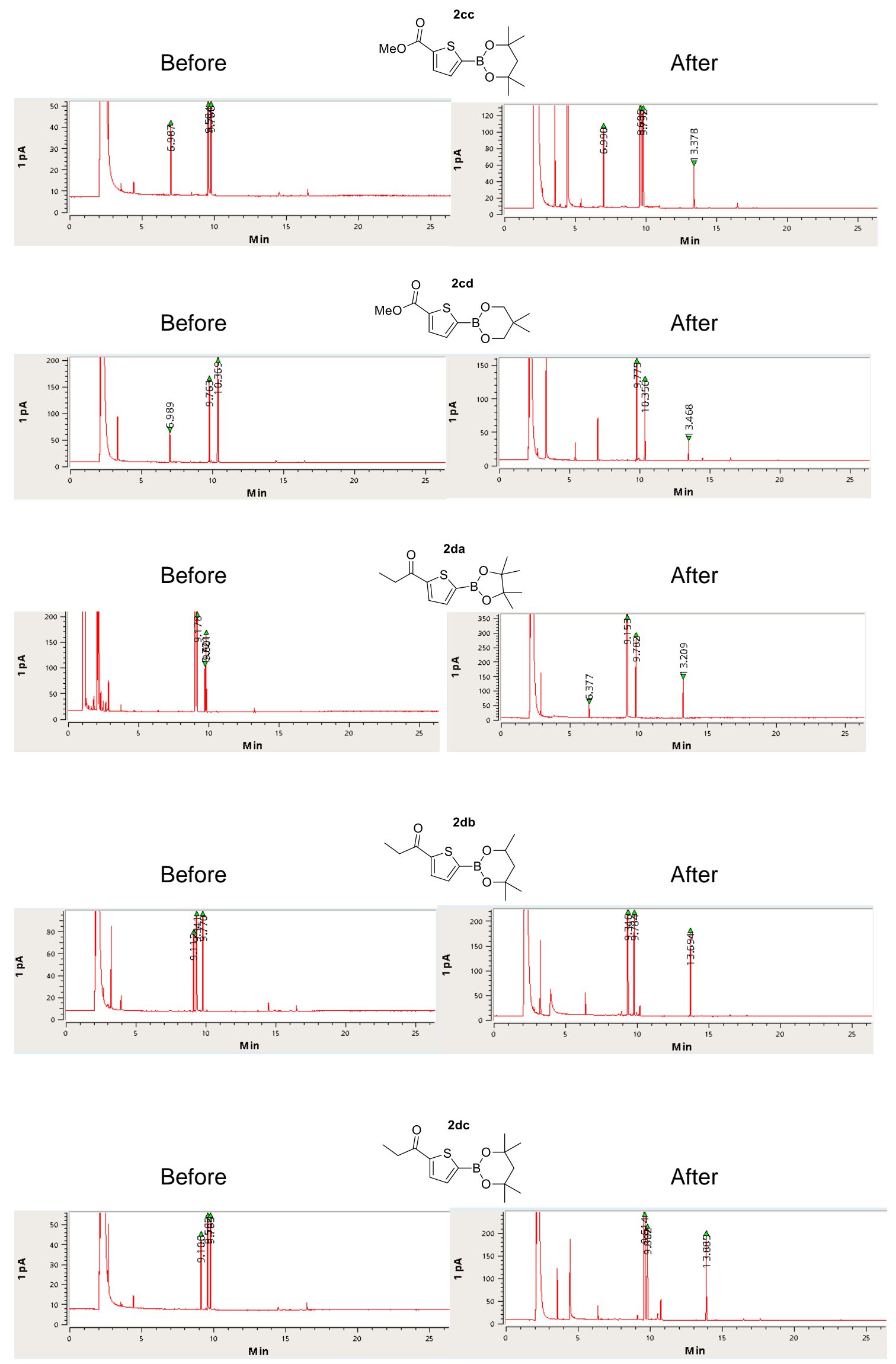

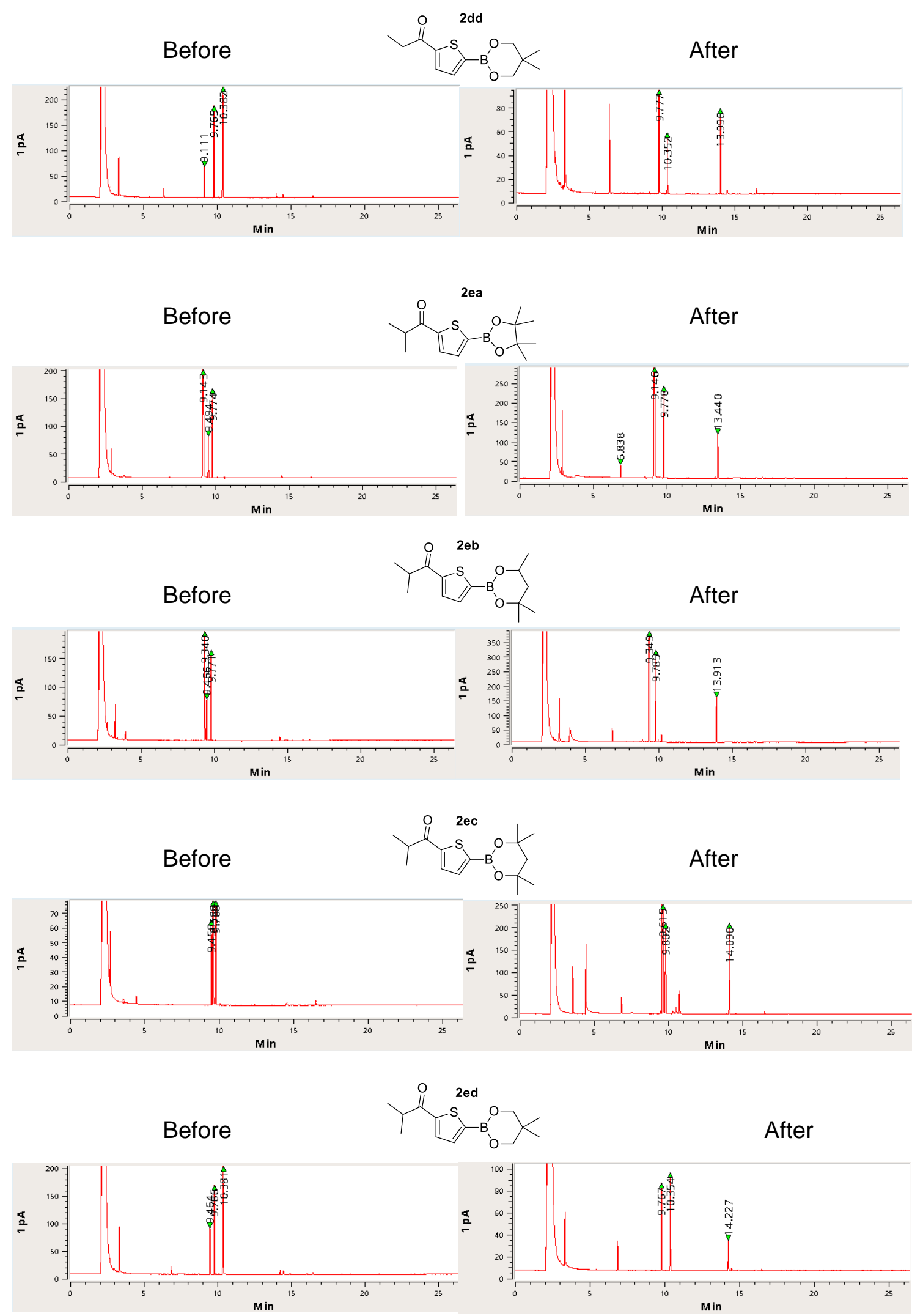


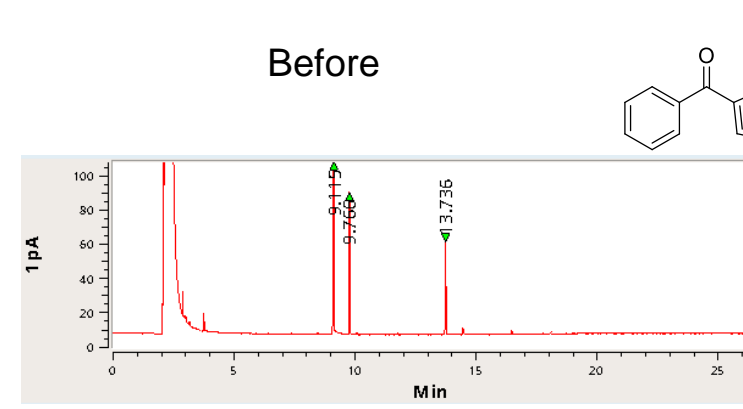

$2 \mathrm{fa}$
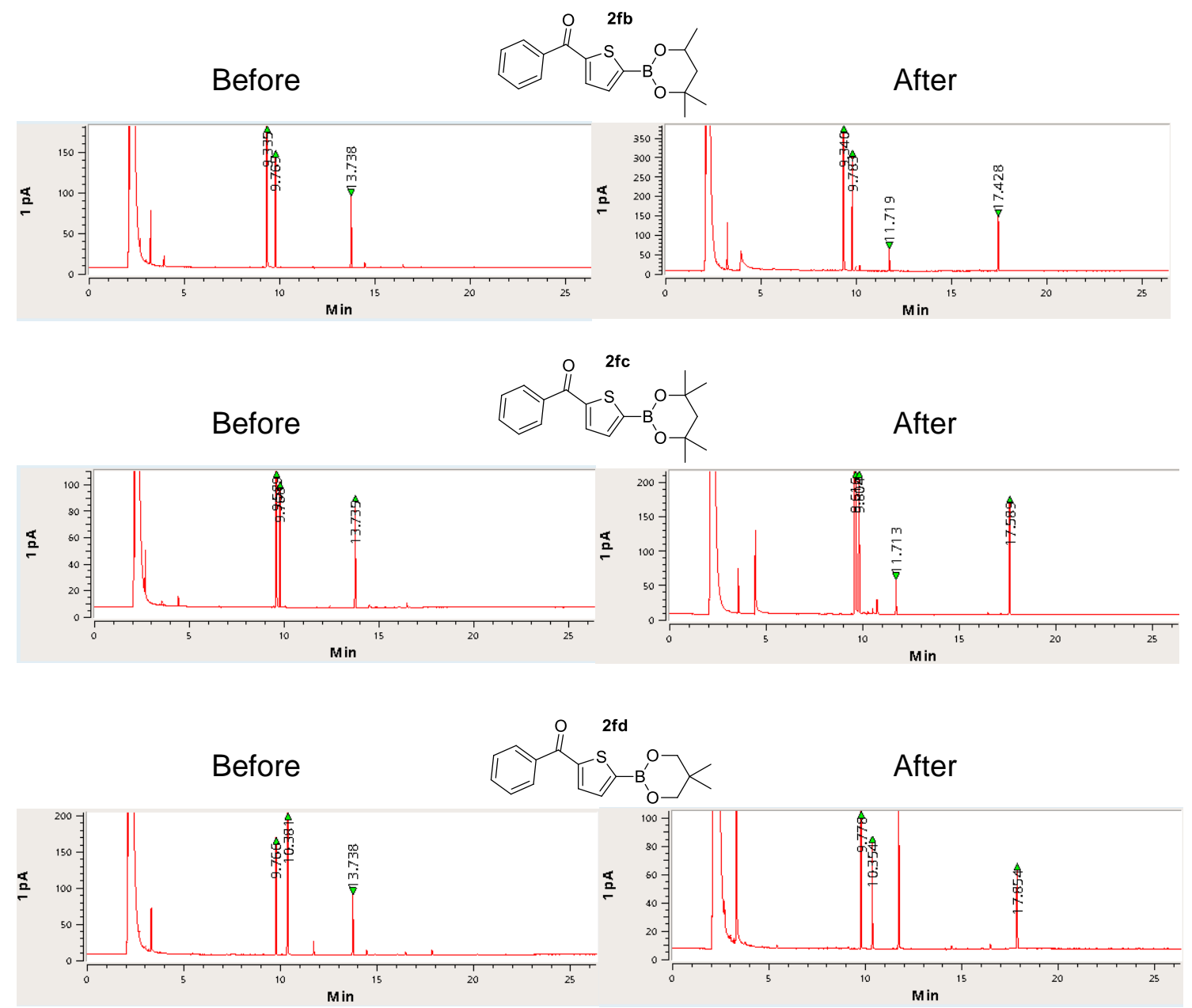

\section{Furans}
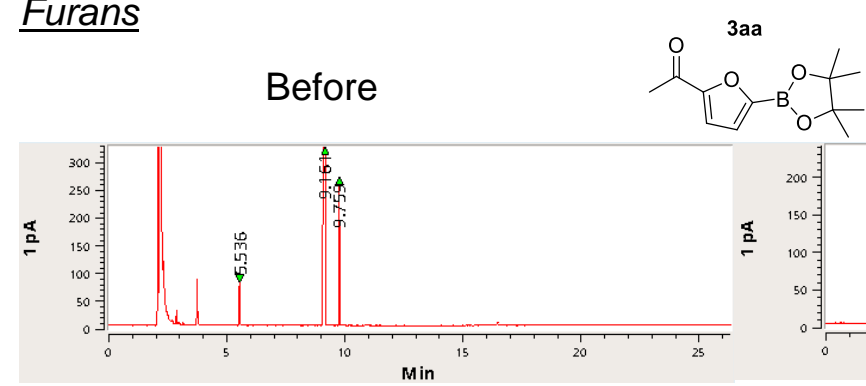

After

$\stackrel{5}{2}$

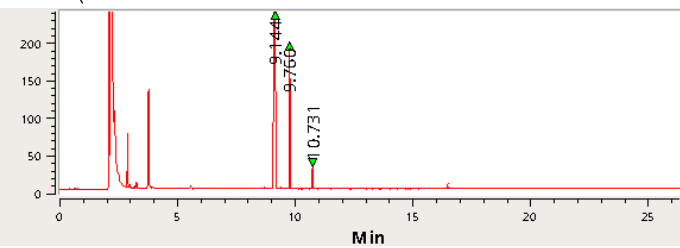



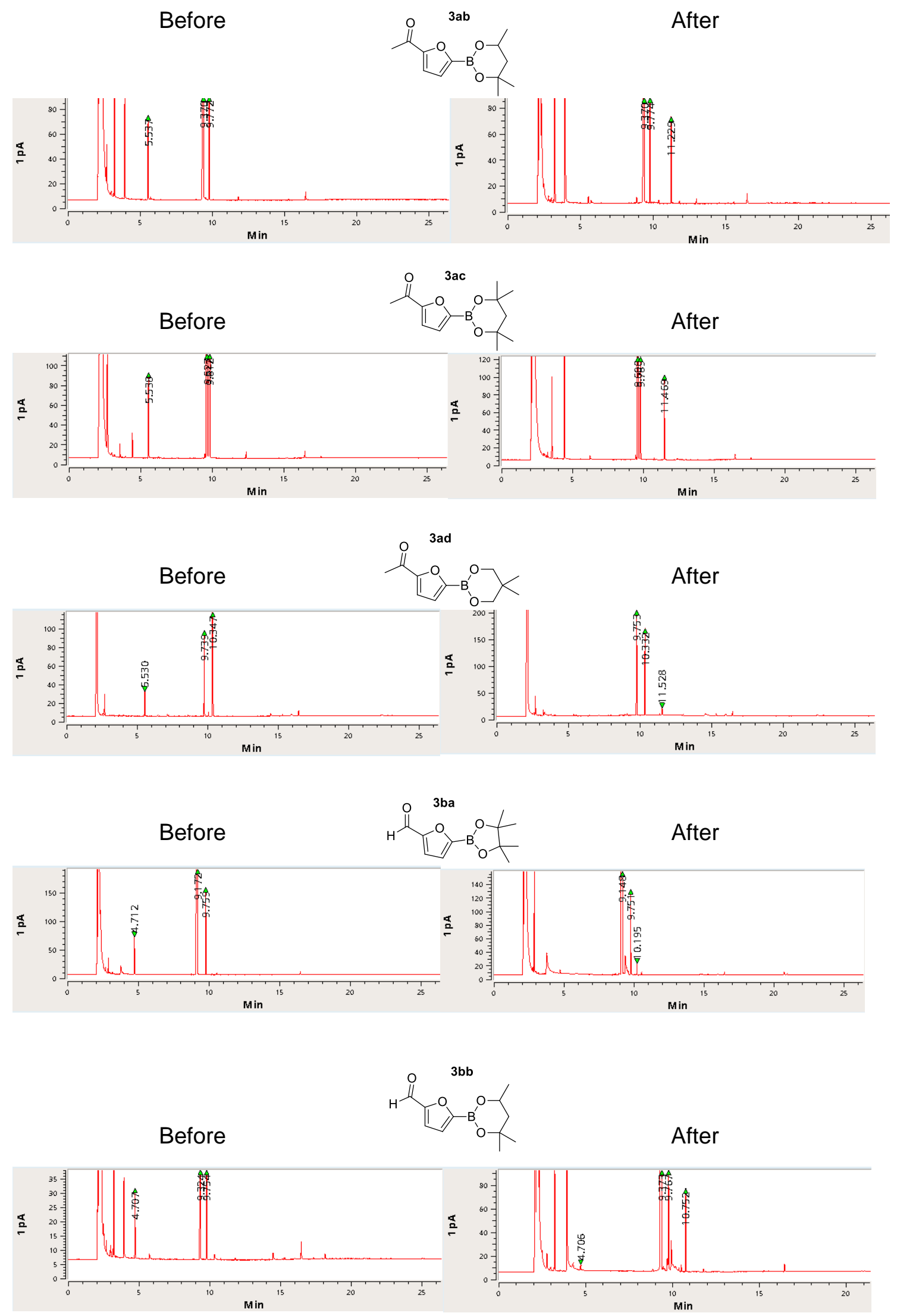

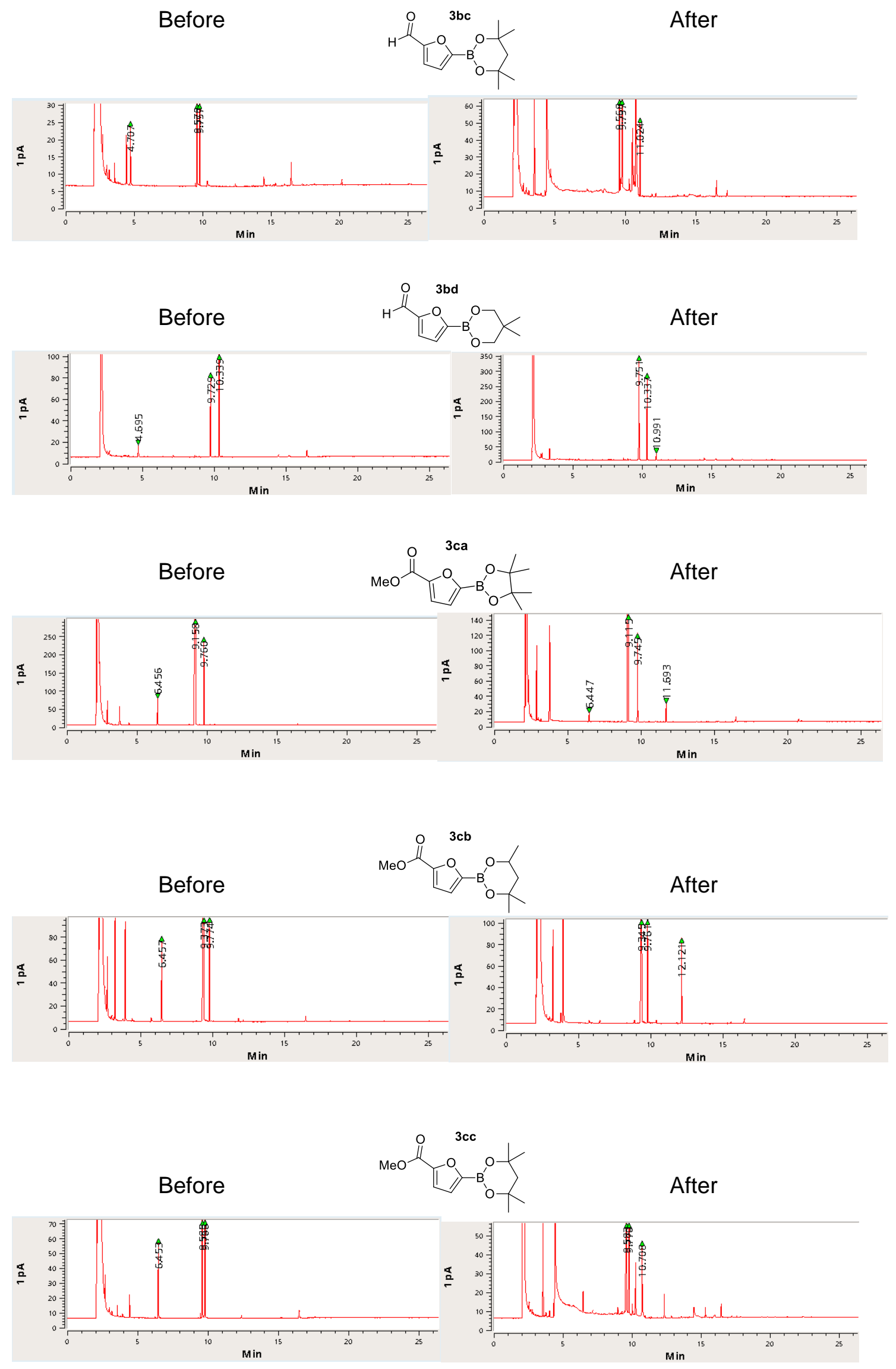


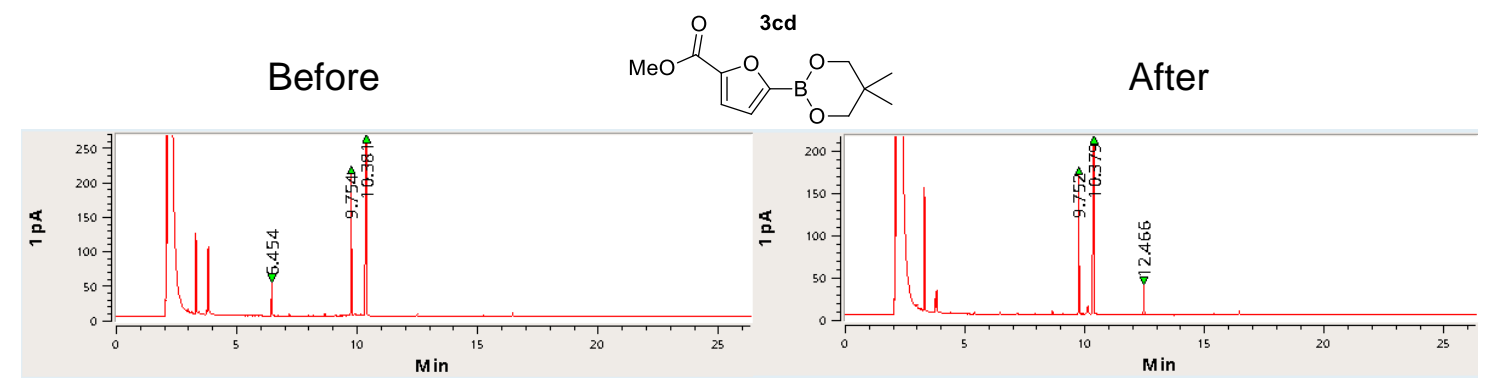

\section{Pyrroles}

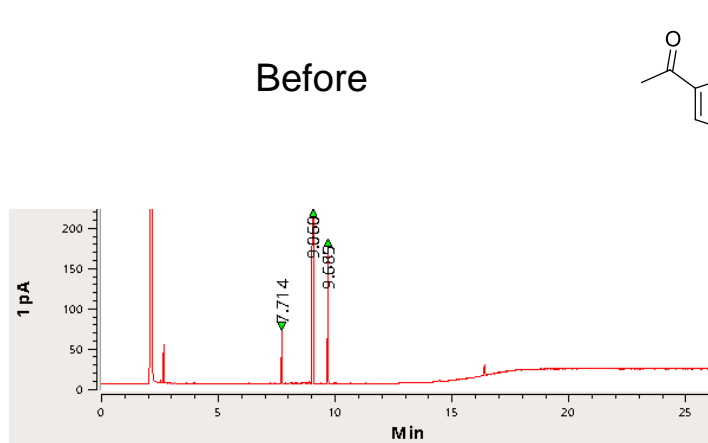

4aa

After
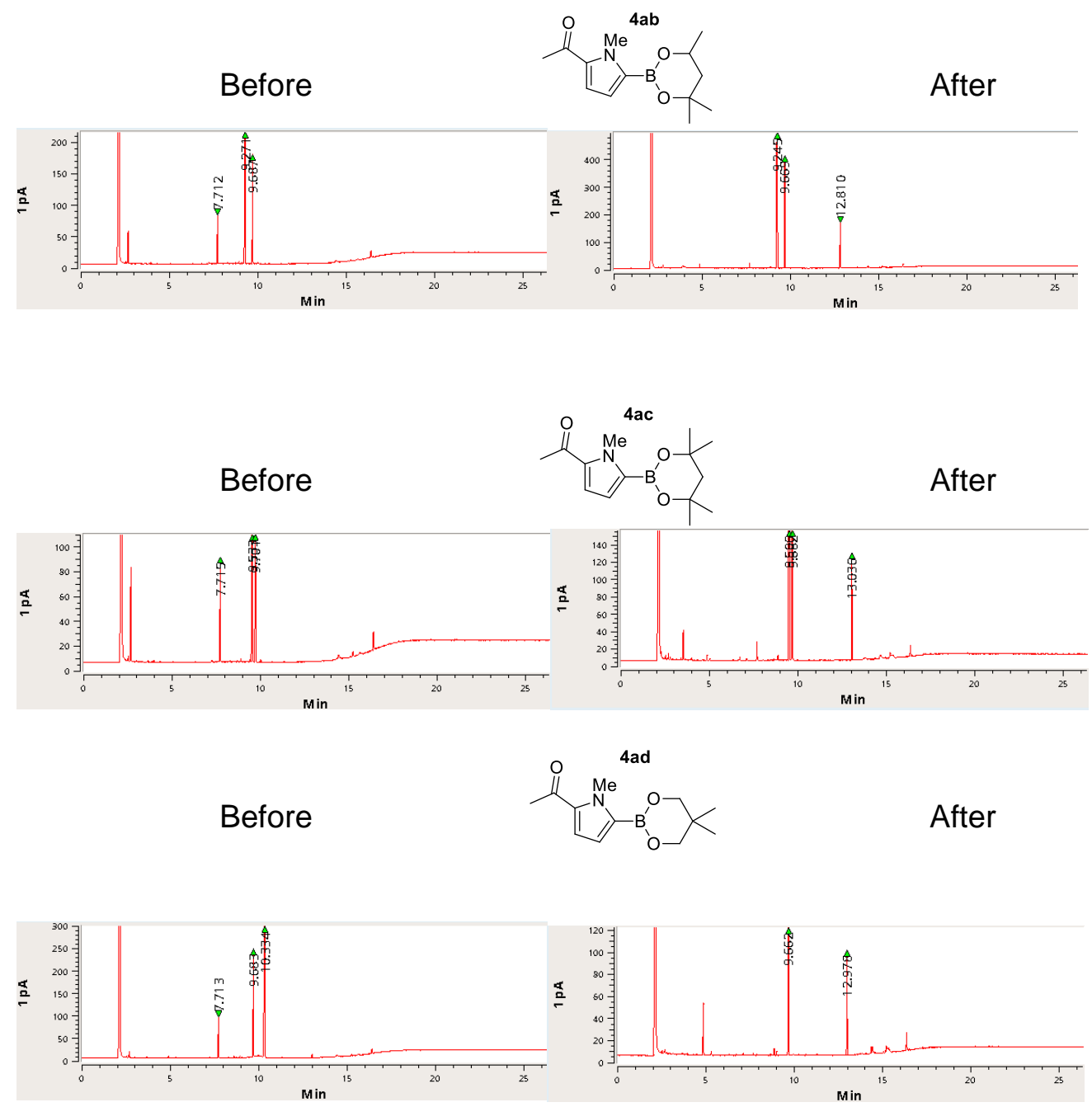

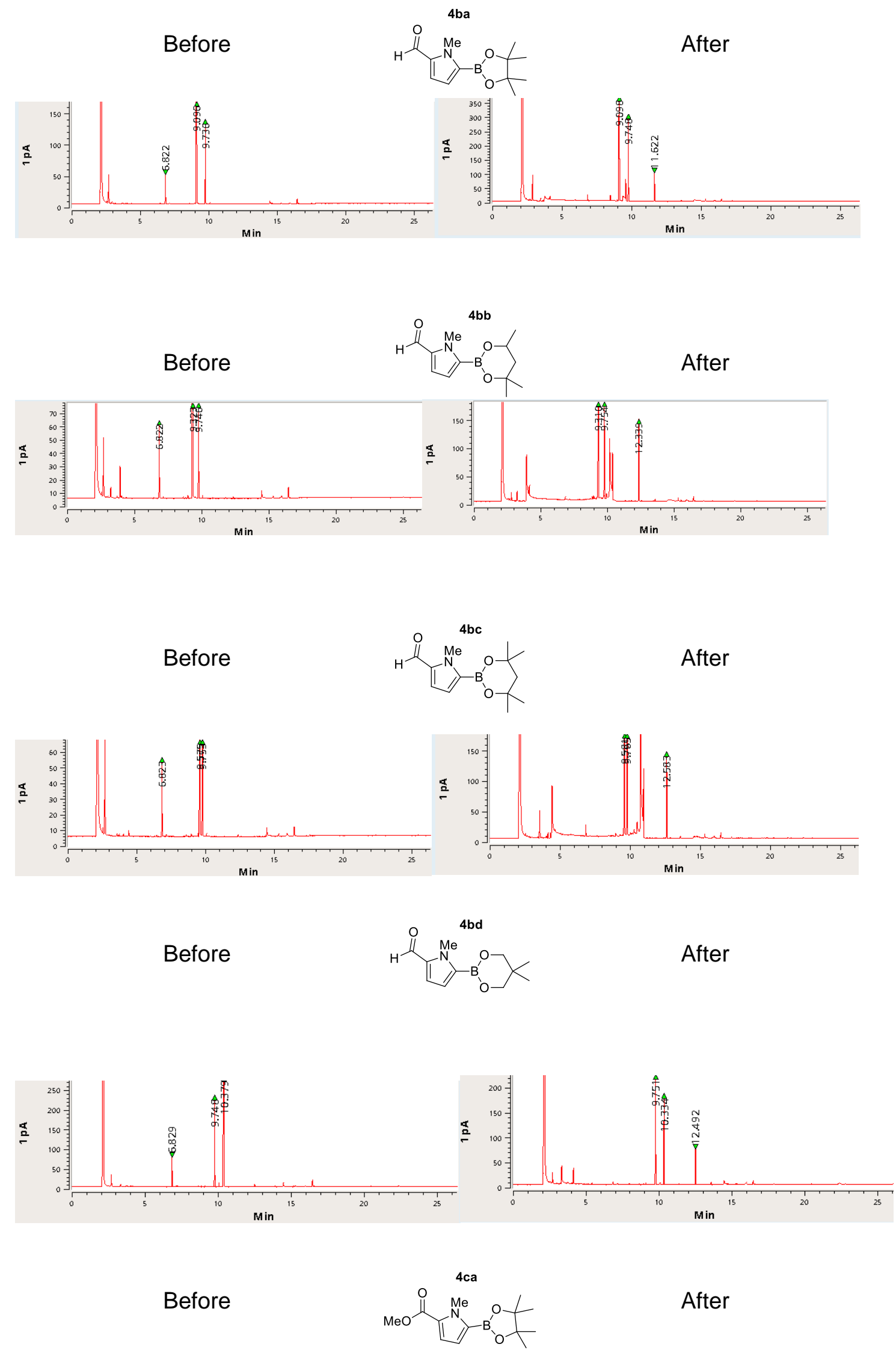

After 

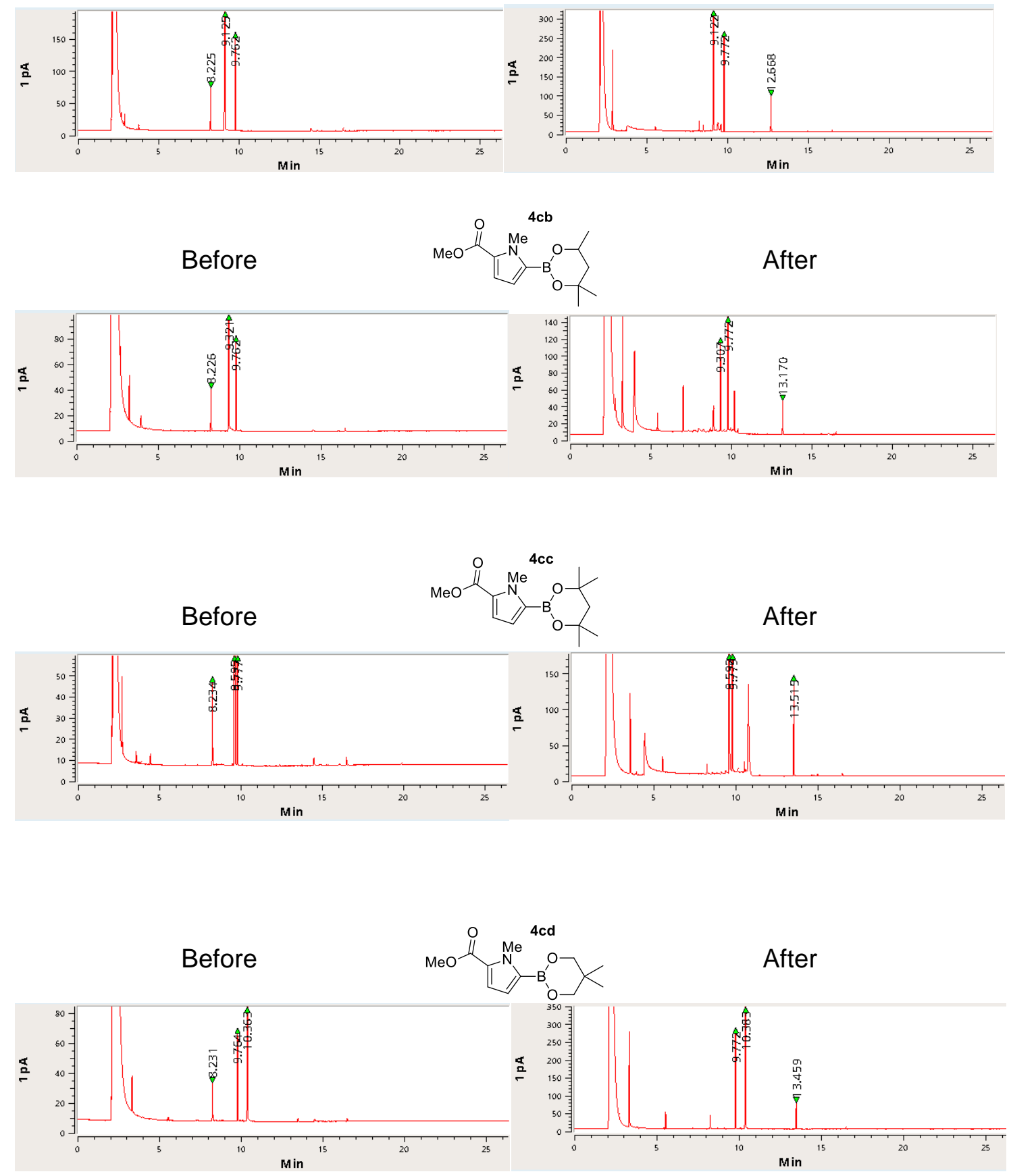

\section{Selenophenes}

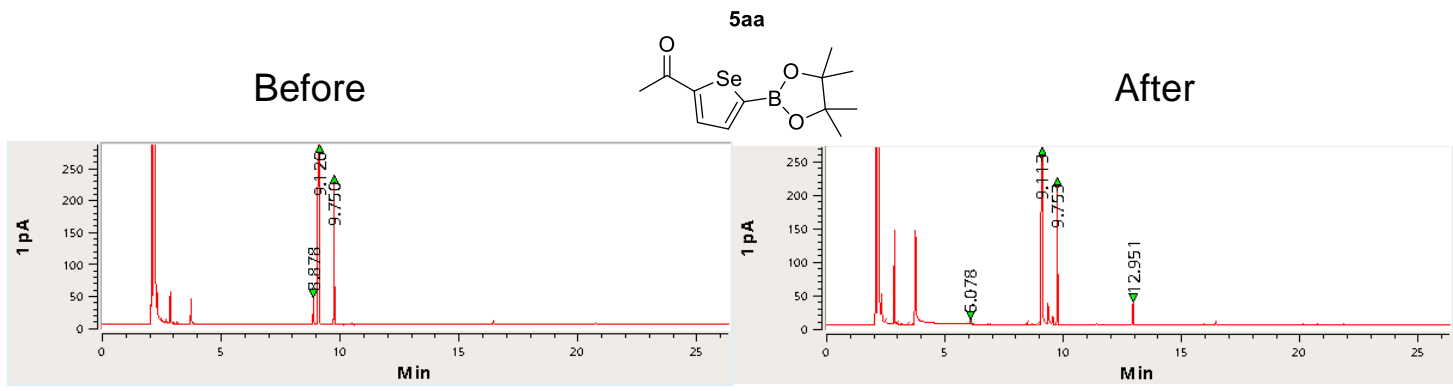



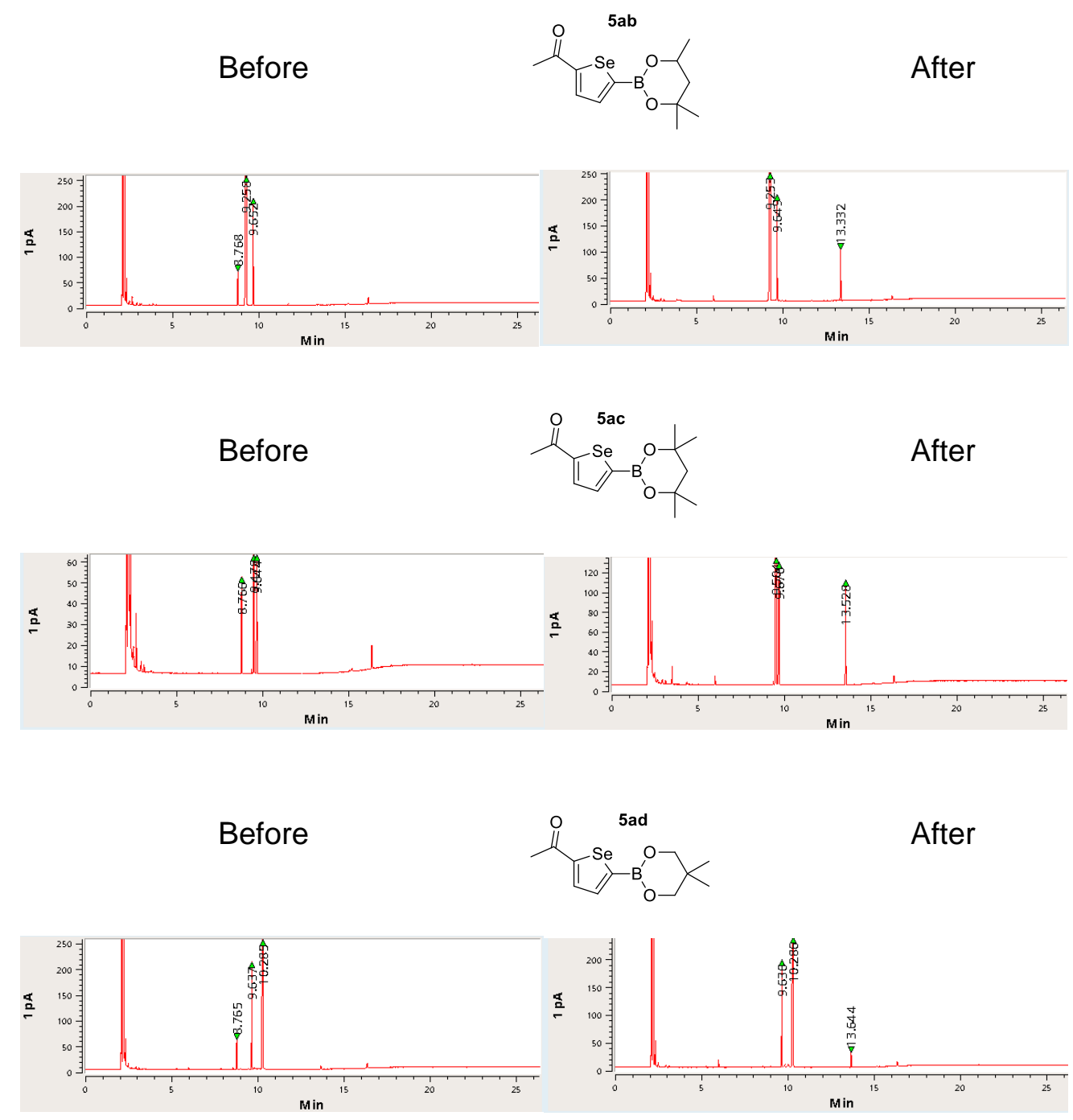


\section{Characterization of compounds}

\section{THIOPHENES}

2aa. 1-(5-(4,4,5,5-tetramethyl-1,3,2-dioxaborolan-2-yl)thiophen-2-yl)ethenone<smiles>CC(=O)c1ccc(B2OC(C)(C)C(C)(C)O2)s1</smiles>

The compound (CAS 942070-32-8) was prepared according to the general procedure using 1-(5-chlorothiophen-2-yl)ethenone (3.2 mg, $20 \mu \mathrm{mol}, 1.0$ equiv.), bis(pinacolato)diboron (50.6 mg, $200 \mu \mathrm{mol}, 10.0$ equiv.), dodecanenitrile $(4.4 \mu \mathrm{L}, 20 \mu \mathrm{mol}, 1.0$ equiv.) as internal standard and DIPEA ( $4.2 \mu \mathrm{L}, 24 \mu \mathrm{mol}, 1.2$ equiv.) and $\mathrm{G} 1$ (10 g/L). The reaction mixture was irradiated for 2 hours, obtaining $72 \%$ product yield according to GC-FID analysis (63\% isolated yield as white powder).

${ }^{1} \mathrm{H}$ NMR $\left(400 \mathrm{MHz}, \mathrm{CDCl}_{3}\right) \delta 7.72(\mathrm{~d}, J=3.7 \mathrm{~Hz}, 1 \mathrm{H}), 7.58(\mathrm{~d}, J=3.7 \mathrm{~Hz}, 1 \mathrm{H}), 2.57$ (s, $3 \mathrm{H}), 1.35$ (s, 12H) ppm.

${ }^{13} \mathrm{C}$ NMR (101 MHz, CDCl $\left.{ }_{3}\right) \delta 190.8(\mathrm{C}), 149.6(\mathrm{C}), 137.4(\mathrm{CH}), 132.8(\mathrm{CH}), 84.8(\mathrm{C})$, $27.6\left(\mathrm{CH}_{3}\right), 24.9\left(\mathrm{CH}_{3}\right)$.

Spectral data were consistent with literature. ${ }^{1}$

\section{2ab. 1-(5-(4,4,6-trimethyl-1,3,2-dioxaborinan-2-yl)thiophen-2-yl)ethenone}<smiles>CC(=O)c1ccc(B2OC(C)CC(C)(C)O2)s1</smiles>

The compound (CAS 2414250-10-3) was prepared according to the general procedure using 1-(5-chlorothiophen-2-yl)ethenone (3.2 mg, $20 \mu \mathrm{mol}, 1.0$ equiv.), bis(hexyleneglycolato)diboron (53.0 $\mathrm{mg}, 200 \mu \mathrm{mol}, 10.0$ equiv.), dodecanenitrile $(4.4 \mu \mathrm{L}, 20 \mu \mathrm{mol}, 1.0$ equiv.) as internal standard and DIPEA $(4.2 \mu \mathrm{L}, 24 \mu \mathrm{mol}, 1.2$ equiv.) and $\mathrm{G} 1$ (10 g/L). The reaction mixture was irradiated for 4 hours, obtaining $61 \%$ product yield according to GC-FID analysis ( $50 \%$ isolated yield as white powder).

${ }^{1} \mathrm{H}$ NMR $\left(400 \mathrm{MHz}, \mathrm{CDCl}_{3}\right) \delta 7.63(\mathrm{~d}, J=3.7 \mathrm{~Hz}, 1 \mathrm{H}), 7.43$ (d, $\left.J=3.7 \mathrm{~Hz}, 1 \mathrm{H}\right), 4.29$ (ddd, $J=11.6,6.1,2.9 \mathrm{~Hz}, 1 \mathrm{H}), 2.50(\mathrm{~s}, 3 \mathrm{H}), 1.82(\mathrm{dd}, J=14.0,2.9 \mathrm{~Hz}, 1 \mathrm{H}), 1.56(\mathrm{dd}, J=$ 13.9, $11.7 \mathrm{~Hz}, 1 \mathrm{H}), 1.31$ (s, 6H), 1.28 (d, J = 6.2 Hz, 3H) ppm.

${ }^{13} \mathrm{C}$ NMR (101 MHz, CDCl $)_{3} \delta 190.8(\mathrm{C}), 148.2(\mathrm{C}), 135.5(\mathrm{CH}), 132.7(\mathrm{CH}), 72.1(\mathrm{C})$, $65.8(\mathrm{CH}), 45.6\left(\mathrm{CH}_{2}\right), 31.1\left(\mathrm{CH}_{3}\right), 28.0\left(\mathrm{CH}_{3}\right), 27.7\left(\mathrm{CH}_{3}\right), 23.0\left(\mathrm{CH}_{3}\right) \mathrm{ppm}$.

Spectral data were consistent with literature. ${ }^{1}$

\section{2ac. 1-(5-(4,4,6,6-tetramethyl-1,3,2-dioxaborinan-2-yl)thiophen-2-yl)ethenone}<smiles>CC(=O)c1ccc(B2OC(C)(C)CC(C)(C)O2)s1</smiles>

The compound (CAS 2414250-15-8) was prepared according to the general procedure using 1-(5-chlorothiophen-2-yl)ethenone (3.2 mg, $20 \mu \mathrm{mol}, 1.0$ equiv.), 4,4,4',4',6,6,6',6'-octamethyl-2,2'bi(1,3,2-dioxaborinane) (56.4 mg, $200 \mu$ mol, 10.0 equiv.), dodecanenitrile $(4.4 \mu \mathrm{L}, 20 \mu \mathrm{mol}, 1.0$ equiv.) as internal standard and DIPEA (4.2 $\mu \mathrm{L}, 24 \mu \mathrm{mol}, 1.2$ equiv.) and $\mathrm{G} 1$ (10 g/L). The reaction mixture was irradiated for 3 hours, obtaining 63\% product yield according to GC-FID analysis (50\% isolated yield as white powder).

${ }^{1} \mathrm{H}$ NMR $\left(400 \mathrm{MHz}, \mathrm{CDCl}_{3}\right) \delta 7.68(\mathrm{~d}, J=3.7 \mathrm{~Hz}, 1 \mathrm{H}), 7.50(\mathrm{~d}, J=3.7 \mathrm{~Hz}, 1 \mathrm{H}), 2.55(\mathrm{~s}$, $3 \mathrm{H}), 1.92(\mathrm{~s}, 2 \mathrm{H}), 1.41(\mathrm{~s}, 12 \mathrm{H}) \mathrm{ppm}$.

${ }^{1}$ J. C. Herrera-Luna, D. Sampedro, M. C. Jiménez and R. Pérez-Ruiz, Organic Letters, 2020, 22, 3273-3278. 
${ }^{13} \mathrm{C}$ NMR (101 MHz, $\left.\mathrm{CDCl}_{3}\right) \delta 191.0(\mathrm{C}), 148.3(\mathrm{C}), 135.5(\mathrm{CH}), 132.8(\mathrm{CH}), 71.9(\mathrm{C})$, $49.1\left(\mathrm{CH}_{2}\right), 31.8\left(\mathrm{CH}_{3}\right), 27.5\left(\mathrm{CH}_{3}\right)$ ppm.

Spectral data were consistent with literature. ${ }^{1}$

\section{2ad. 1-(5-(5,5-dimethyl-1,3,2-dioxaborinan-2-yl)thiophen-2-yl)ethenone}<smiles>CC(=O)c1ccc(B2OCC(C)(C)CO2)s1</smiles>

The compound (CAS 2414250-20-5) was prepared according to the general procedure using 1-(5-chlorothiophen-2-yl)ethenone (3.2 $\mathrm{mg}, 20 \mu \mathrm{mol}, 1.0$ equiv.), bis(neopentylglycolato)diboron (45.2 mg, $200 \mu \mathrm{mol}, 10.0$ equiv.), dodecanenitrile $(4.4 \mu \mathrm{L}, 20$ $\mu \mathrm{mol}, 1.0$ equiv.) as internal standard and DIPEA (4.2 $\mu \mathrm{L}, 24 \mu \mathrm{mol}, 1.2$ equiv.) and G1 $(10 \mathrm{~g} / \mathrm{L})$. The reaction mixture was irradiated for 3 hours, obtaining $50 \%$ product yield according to GC-FID analysis ( $45 \%$ isolated yield as white powder).

${ }^{1} \mathrm{H}$ NMR $\left(400 \mathrm{MHz}, \mathrm{CDCl}_{3}\right) \delta 7.69(\mathrm{~d}, J=3.7 \mathrm{~Hz}, 1 \mathrm{H}), 7.50(\mathrm{~d}, J=3.7 \mathrm{~Hz}, 1 \mathrm{H}), 3.75(\mathrm{~s}$, $4 \mathrm{H}), 2.55(\mathrm{~s}, 3 \mathrm{H}), 1.01(\mathrm{~s}, 6 \mathrm{H}) \mathrm{ppm}$.

${ }^{13} \mathrm{C}$ NMR $\left(101 \mathrm{MHz}, \mathrm{CDCl}_{3}\right) \delta 190.9(\mathrm{C}), 148.7(\mathrm{C}), 135.9(\mathrm{CH}), 132.9(\mathrm{CH}), 73.1\left(\mathrm{CH}_{2}\right)$, $32.1(\mathrm{C}), 27.5\left(\mathrm{CH}_{3}\right), 21.9\left(\mathrm{CH}_{3}\right) \mathrm{ppm}$.

Spectral data were consistent with literature. ${ }^{1}$

\section{1-(5-(phenylthio)thiophen-2-yl)ethenone}

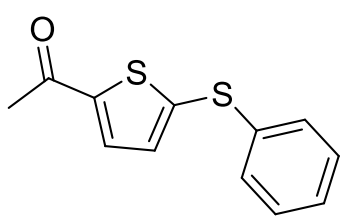

The compound (CAS: 90680-26-5) was prepared according to the general procedure using 1-(5-chlorothiophen-2-yl)ethenone (3.2 $\mathrm{mg}, 20 \mu \mathrm{mol}, 1.0$ equiv.), bis(pinacolato)diboron (50.6 mg, 200 $\mu \mathrm{mol}, 10.0$ equiv.), diphenyldisulfide ( $4.4 \mathrm{mg}, 80 \mu \mathrm{mol}, 1.0$ equiv.) dodecanenitrile $(4.4 \mu \mathrm{L}, 20 \mu \mathrm{mol}, 1.0$ equiv.) as internal standard and DIPEA (4.2 $\mu \mathrm{L}, 24 \mu \mathrm{mol}, 1.2$ equiv.) and $\mathrm{G} 1$ (10g/L). The reaction mixture was irradiated for 3 hours, obtaining $78 \%$ product yield according to GC-FID analysis.

${ }^{1} \mathrm{H}$ NMR $\left(400 \mathrm{MHz}, \mathrm{CDCl}_{3}\right) \delta 7.56(\mathrm{~d}, J=3.9 \mathrm{~Hz}, 1 \mathrm{H}), 7.43-7.38(\mathrm{~m}, 2 \mathrm{H}), 7.36-7.28$ $(\mathrm{m}, 3 \mathrm{H}), 7.10(\mathrm{~d}, J=3.9 \mathrm{~Hz}, 1 \mathrm{H}), 2.50(\mathrm{~s}, 3 \mathrm{H}) \mathrm{ppm}$.

${ }^{13} \mathrm{C}$ NMR (101 MHz, $\left.\mathrm{CDCl}_{3}\right) \delta 190.0(\mathrm{C}), 146.3(\mathrm{C}), 145.7(\mathrm{C}), 135.2(\mathrm{C}), 132.8(\mathrm{CH})$, $132.1(\mathrm{CH}), 130.9(\mathrm{CH}), 129.6(\mathrm{CH}), 128.2(\mathrm{CH}), 26.6\left(\mathrm{CH}_{3}\right) \mathrm{ppm}$.

Spectral data were consistent with literature. ${ }^{1}$

\section{2ba. 5-(4,4,5,5-tetramethyl-1,3,2-dioxaborolan-2-yl)thiophene-2-carbaldehyde}<smiles>CC1(C)OB(c2ccc(C=O)s2)OC1(C)C</smiles>

The compound (CAS 1040281-83-1) was prepared according to the general procedure using 5-bromothiophene-2-carbaldehyde (2.5 $\mu \mathrm{L}, 20 \mu \mathrm{mol}, 1.0$ equiv.), bis(pinacolato)diboron (50.6 mg, $200 \mu \mathrm{mol}, 10.0$ equiv.), dodecanenitrile $(4.4 \mu \mathrm{L}, 20 \mu \mathrm{mol}, 1.0$ equiv.) as internal standard and DIPEA ( $4.2 \mu \mathrm{L}, 24 \mu \mathrm{mol}, 1.2$ equiv.) and G1 (10 g/L). The reaction mixture was irradiated for 3 hours, obtaining $66 \%$ product yield according to GC-FID analysis ( $52 \%$ isolated yield as white powder).

${ }^{1} \mathrm{H}$ NMR $\left(400 \mathrm{MHz}, \mathrm{CDCl}_{3}\right) \delta 9.97(\mathrm{~s}, 1 \mathrm{H}), 7.79(\mathrm{~d}, J=3.7 \mathrm{~Hz}, 1 \mathrm{H}), 7.65(\mathrm{~d}, J=3.7 \mathrm{~Hz}$, $1 \mathrm{H}), 1.35(\mathrm{~s}, 12 \mathrm{H})$.

${ }^{13} \mathrm{C}$ NMR (101 MHz, $\left.\mathrm{CDCl}_{3}\right) \delta 183.1(\mathrm{C}), 149.1(\mathrm{C}), 137.4(\mathrm{CH}), 136.2(\mathrm{CH}), 85.0(\mathrm{C})$, $24.9\left(\mathrm{CH}_{3}\right) \mathrm{ppm}$.

Spectral data were consistent with literature. ${ }^{1}$ 
2bb. 5-(4,4,6-trimethyl-1,3,2-dioxaborinan-2-yl)thiophene-2-carbaldehyde<smiles>CC1CC(C)(C)OB(c2ccc(C=O)s2)O1</smiles>

The compound (CAS 2414250-11-4) was prepared according to the general procedure using 5-bromothiophene-2-carbaldehyde $(2.5 \mu \mathrm{L}, 20 \mu \mathrm{mol}, 1.0$ equiv.), bis(hexyleneglycolato)diboron (53.0 $\mathrm{mg}, 200 \mu \mathrm{mol}, 10.0$ equiv.), dodecanenitrile $(4.4 \mu \mathrm{L}, 20 \mu \mathrm{mol}, 1.0$ equiv.) as internal standard and DIPEA $(4.2 \mu \mathrm{L}, 24 \mu \mathrm{mol}, 1.2$ equiv.) and $\mathrm{G} 1$ (10 g/L). The reaction mixture was irradiated for 3 hours, obtaining $62 \%$ product yield according to GC-FID analysis ( $49 \%$ isolated yield as white powder).

${ }^{1} \mathrm{H}$ NMR $\left(400 \mathrm{MHz}, \mathrm{CDCl}_{3}\right) \delta 9.87(\mathrm{~s}, 1 \mathrm{H}), 7.69(\mathrm{~d}, J=3.7 \mathrm{~Hz}, 1 \mathrm{H}), 7.49(\mathrm{~d}, J=3.7 \mathrm{~Hz}$, $1 \mathrm{H}), 4.29$ (ddd, $J=11.6,6.1,2.9 \mathrm{~Hz}, 1 \mathrm{H}), 1.82(\mathrm{dd}, J=14.1,2.9 \mathrm{~Hz}, 1 \mathrm{H}), 1.60-1.51$ $(\mathrm{m}, 1 \mathrm{H}), 1.30(\mathrm{~s}, 6 \mathrm{H}), 1.27(\mathrm{~d}, J=6.2 \mathrm{~Hz}, 3 \mathrm{H}) \mathrm{ppm}$.

${ }^{13} \mathrm{C}$ NMR (101 MHz, CDCl $\left.{ }_{3}\right) \delta 183.1(\mathrm{C}), 147.8(\mathrm{C}), 136.2(\mathrm{CH}), 135.5(\mathrm{CH}), 71.9(\mathrm{C})$, $65.7(\mathrm{CH}), 45.6\left(\mathrm{CH}_{2}\right), 31.0\left(\mathrm{CH}_{3}\right), 27.7\left(\mathrm{CH}_{3}\right), 22.9\left(\mathrm{CH}_{3}\right) \mathrm{ppm}$.

Spectral data were consistent with literature. ${ }^{1}$

\section{2bc. 5-(4,4,6,6-tetramethyl-1,3,2-dioxaborinan-2-yl)thiophene-2-carbaldehyde}<smiles>CC1(C)CC(C)(C)OB(c2ccc(C=O)s2)O1</smiles>

The compound (CAS 2414250-16-9) was prepared according to the general procedure using 5-bromothiophene-2-carbaldehyde (2.5 $\mu \mathrm{L}, 20 \mu \mathrm{mol}, 1.0$ equiv.), 4,4,4',4',6,6,6',6'-octamethyl-2,2'bi(1,3,2-dioxaborinane) (56.4 mg, $200 \mu \mathrm{mol}, 10.0$ equiv.), dodecanenitrile $(4.4 \mu \mathrm{L}, 20 \mu \mathrm{mol}, 1.0$ equiv.) as internal standard and DIPEA ( $4.2 \mu \mathrm{L}, 24 \mu \mathrm{mol}, 1.2$ equiv.) and G1 (10 g/L). The reaction mixture was irradiated for 5 hours, obtaining $89 \%$ product yield according to GC-FID analysis (73\% isolated yield as white powder).

${ }^{1} \mathrm{H}$ NMR $\left(400 \mathrm{MHz}, \mathrm{CDCl}_{3}\right) \delta 9.93(\mathrm{~s}, 1 \mathrm{H}), 7.75(\mathrm{~d}, J=3.6 \mathrm{~Hz}, 1 \mathrm{H}), 7.57(\mathrm{~d}, J=3.6 \mathrm{~Hz}$, $1 \mathrm{H}), 1.92(\mathrm{~s}, 2 \mathrm{H}), 1.41(\mathrm{~s}, 12 \mathrm{H}) \mathrm{ppm}$.

${ }^{13} \mathrm{C}$ NMR (101 MHz, CDCl $\left.{ }_{3}\right) \delta 183.3(\mathrm{C}), 147.9(\mathrm{C}), 136.3(\mathrm{CH}), 135.5(\mathrm{CH}), 72.0(\mathrm{C})$, $49.1\left(\mathrm{CH}_{2}\right), 31.7\left(\mathrm{CH}_{3}\right)$ ppm.

Spectral data were consistent with literature. ${ }^{1}$

\section{2bd. 5-(5,5-dimethyl-1,3,2-dioxaborinan-2-yl)thiophene-2-carbaldehyde}<smiles>CC1(C)COB(c2ccc(C=O)s2)OC1</smiles>

The compound (CAS 2414250-21-6) was prepared according to the general procedure using 5-bromothiophene-2-carbaldehyde (2.5 $\mu \mathrm{L}, 20 \mu \mathrm{mol}, 1.0$ equiv.), bis(neopentylglycolato)diboron (45.2 mg, $200 \mu \mathrm{mol}, 10.0$ equiv.), dodecanenitrile (4.4 $\mu \mathrm{L}, 20$ $\mu \mathrm{mol}, 1.0$ equiv.) as internal standard and DIPEA ( $4.2 \mu \mathrm{L}, 24$ umol, 1.2 equiv.) and $\mathrm{G} 1$ (10 g/L). The reaction mixture was irradiated for 2 hours, obtaining $56 \%$ product yield according to GC-FID analysis ( $41 \%$ isolated yield as white powder).

${ }^{1} \mathrm{H}$ NMR $\left(400 \mathrm{MHz}, \mathrm{CDCl}_{3}\right) \delta 9.96(\mathrm{~s}, 1 \mathrm{H}), 7.78(\mathrm{~d}, J=3.7 \mathrm{~Hz}, 1 \mathrm{H}), 7.59(\mathrm{~d}, J=3.7 \mathrm{~Hz}$, $1 \mathrm{H}), 3.78(\mathrm{~s}, 4 \mathrm{H}), 1.03(\mathrm{~s}, 6 \mathrm{H}) \mathrm{ppm}$.

${ }^{13} \mathrm{C}$ NMR $\left(101 \mathrm{MHz}, \mathrm{CDCl}_{3}\right) \delta 183.3(\mathrm{C}), 148.3(\mathrm{C}), 136.4(\mathrm{CH}), 136.0(\mathrm{CH}), 72.7\left(\mathrm{CH}_{2}\right)$, $32.2(\mathrm{C}), 22.0\left(\mathrm{CH}_{3}\right)$ ppm.

Spectral data were consistent with literature. ${ }^{1}$

2ca. Methyl 5-(4,4,5,5-tetramethyl-1,3,2-dioxaborolan-2-yl)thiophene-2-carboxylate 
<smiles>COC(=O)c1ccc(B2OC(C)(C)C(C)(C)O2)s1</smiles>

The compound (CAS 916138-13-1) was prepared according to the general procedure using methyl 5-bromothiophene-2carboxylate $(2.6 \mu \mathrm{L}, \quad 20 \mu \mathrm{mol}, \quad 1.0 \quad$ equiv. $)$, bis(pinacolato)diboron (50.6 mg, $200 \mu \mathrm{mol}, 10.0$ equiv.), dodecanenitrile $(4.4 \mu \mathrm{L}, 20 \mu \mathrm{mol}, 1.0$ equiv.) as internal standard and DIPEA ( $4.2 \mu \mathrm{L}, 24 \mu \mathrm{mol}, 1.2$ equiv.) and G1 (10 g/L). The reaction mixture was irradiated for 20 hours, obtaining $26 \%$ product yield according to GC-FID analysis (19\% isolated yield as white powder).

Spectral data were consistent with literature. ${ }^{2}$

${ }^{1} \mathrm{H}$ NMR $\left(400 \mathrm{MHz}, \mathrm{CDCl}_{3}\right) \delta 7.80(\mathrm{~d}, \mathrm{~J}=3.7 \mathrm{~Hz}, 1 \mathrm{H}), 7.54(\mathrm{~d}, \mathrm{~J}=3.6 \mathrm{~Hz}, 1 \mathrm{H}), 3.88(\mathrm{~s}$, $3 \mathrm{H}), 1.34(\mathrm{~s}, 12 \mathrm{H}) \mathrm{ppm}$.

${ }^{13} \mathrm{C}$ NMR (101 MHz, CDCl $\left.{ }_{3}\right) \delta 162.8(\mathrm{C}), 139.5(\mathrm{C}), 137.0(\mathrm{CH}), 134.1(\mathrm{CH}), 84.7(\mathrm{C})$, $52.4\left(\mathrm{O}-\mathrm{CH}_{3}\right), 24.9\left(\mathrm{CH}_{3}\right) \mathrm{ppm}$.

2cb. Methyl 5-(4,4,6-trimethyl-1,3,2-dioxaborinan-2-yl)thiophene-2-carboxylate<smiles>COC(=O)c1ccc(B2OC(C)CC(C)(C)O2)s1</smiles>

The compound was prepared according to the general procedure using methyl 5-bromothiophene-2-carboxylate (2.6 $\mu \mathrm{L}, 80 \mu \mathrm{mol}, 1.0$ equiv.), bis(hexyleneglycolato)diboron (53.0 $\mathrm{mg}, 200 \mu \mathrm{mol}, 10.0$ equiv.), dodecanenitrile $(4.4 \mu \mathrm{L}, 20 \mu \mathrm{mol}$, 1.0 equiv.) as internal standard and DIPEA $(4.2 \mu \mathrm{L}, 24 \mu \mathrm{mol}$, 1.2 equiv.) and $\mathrm{G} 1$ (10 $\mathrm{g} / \mathrm{L})$. The reaction mixture was irradiated for 19 hours, obtaining $30 \%$ product yield according to GC-FID analysis ( $25 \%$ isolated yield as white powder).

${ }^{1} \mathrm{H}$ NMR $\left(400 \mathrm{MHz}, \mathrm{CDCl}_{3}\right) \delta 7.77$ (d, J = 3.6 Hz, 1H), 7.47 (d, J = 3.6 Hz, 1H), 4.35 (ddd, $\mathrm{J}=11.6,6.0,2.9 \mathrm{~Hz}, 1 \mathrm{H}), 3.87(\mathrm{~s}, 3 \mathrm{H}), 1.87(\mathrm{dd}, \mathrm{J}=14.0,2.9 \mathrm{~Hz}, 1 \mathrm{H}), 1.56-1.44(\mathrm{~m}$, $1 \mathrm{H}), 1.36(\mathrm{~s}, 6 \mathrm{H}), 1.33(\mathrm{~d}, \mathrm{~J}=6.2 \mathrm{~Hz}, 3 \mathrm{H}) \mathrm{ppm}$.

${ }^{13} \mathrm{C}$ NMR (101 MHz, CDCl $)$ ס $163.1(\mathrm{C}), 138.0(\mathrm{C}), 135.2(\mathrm{CH}), 134.0(\mathrm{CH}), 72.0(\mathrm{C})$, $65.7(\mathrm{CH}), 52.2\left(\mathrm{O}-\mathrm{CH}_{3}\right), 46.1\left(\mathrm{CH}_{2}\right) 31.05\left(\mathrm{CH}_{3}\right), 28.2\left(\mathrm{CH}_{3}\right), 21.18\left(\mathrm{CH}_{3}\right) \mathrm{ppm}$.

GC-MS (EI): m/z (relative intensity): 268.1 (40) [M+•], 253.1 (35), 237.1 (20), 169.0 (20), 137.0 (20), 43.1 (100).

\section{2cc. Methyl 5-(4,4,6,6-tetramethyl-1,3,2-dioxaborinan-2-yl)thiophene-2-carboxylate}<smiles>COC(=O)c1ccc(B2OC(C)(C)CC(C)(C)O2)s1</smiles>

The compound was prepared according to the general procedure using methyl 5-bromothiophene-2-carboxylate $(2.6$ $\mu \mathrm{L}, 80 \mu \mathrm{mol}, 1.0$ equiv.), 4,4,4',4',6,6,6',6'-octamethyl-2,2'bi(1,3,2-dioxaborinane) (56.4 mg, $200 \mu \mathrm{mol}, 10.0$ equiv.), dodecanenitrile $(4.4 \mu \mathrm{L}, 20 \mu \mathrm{mol}, 1.0$ equiv.) as internal standard and DIPEA ( $4.2 \mu \mathrm{L}, 24 \mu \mathrm{mol}, 1.2$ equiv.) and $\mathrm{G} 1$ (10 g/L). The reaction mixture was irradiated for 5 hours, obtaining $23 \%$ product yield according to GC-FID analysis (15\% isolated yield as white powder).

${ }^{1} \mathrm{H}$ NMR $\left(400 \mathrm{MHz}, \mathrm{CDCl}_{3}\right) \delta 7.77(\mathrm{~d}, \mathrm{~J}=3.6 \mathrm{~Hz}, 1 \mathrm{H}), 7.48(\mathrm{~d}, \mathrm{~J}=3.6 \mathrm{~Hz}, 1 \mathrm{H}), 3.87(\mathrm{~s}$, $3 \mathrm{H}), 1.92(\mathrm{~s}, 2 \mathrm{H}), 1.41(\mathrm{~s}, 12 \mathrm{H}) \mathrm{ppm}$.

${ }^{13} \mathrm{C}$ NMR (101 MHz, CDCl$\left.)_{3}\right) \delta 163.03(\mathrm{C}), 137.96(\mathrm{C}), 135.18(\mathrm{CH}), 134.00(\mathrm{CH}), 119.97$ (C), $71.81(\mathrm{C}), 52.17\left(\mathrm{O}-\mathrm{CH}_{3}\right), 49.14\left(\mathrm{CH}_{2}\right), 31.76\left(\mathrm{CH}_{3}\right) \mathrm{ppm}$.

\footnotetext{
${ }^{2}$ Ghayoor A. Chotana, Venkata A. Kallepalli, Robert E. Maleczka Jr., Milton R. Smith III. Tetrahedron 2008, 64, 6103-6114.
} 
GC-MS (EI): m/z (relative intensity): 282.1 (100) [M+•], 267.1 (100), 251.1 (30), 183.0 (50), 169.0 (50), 137.0 (60), 99.1 (40), 56.1 (80), 43.1 (80).

2cd. Methyl 5-(5,5-dimethyl-1,3,2-dioxaborinan-2-yl)thiophene-2carboxylatecarboxylate<smiles>COC(=O)c1ccc(B2OCC(C)(C)CO2)s1</smiles>

The compound was prepared according to the general procedure using methyl 5-bromothiophene-2-carboxylate (2.6 $\mu \mathrm{L}, 80 \mu \mathrm{mol}, 1.0$ equiv.), bis(neopentylglycoltao)diboron (45.2 mg, $200 \mu \mathrm{mol}, 10.0$ equiv.), dodecanenitrile $(4.4 \mu \mathrm{L}, 20$ $\mu \mathrm{mol}, 1.0$ equiv.) as internal standard and DIPEA ( $4.2 \mu \mathrm{L}, 24 \mu \mathrm{mol}, 1.2$ equiv.) and G1 $(10 \mathrm{~g} / \mathrm{L})$. The reaction mixture was irradiated for 5 hours, obtaining $24 \%$ product yield according to GC-FID analysis (16\% isolated yield as rose-pale powder).

${ }^{1} \mathrm{H}$ NMR $\left(400 \mathrm{MHz}, \mathrm{CDCl}_{3}\right) \delta 7.79(\mathrm{~d}, \mathrm{~J}=3.6 \mathrm{~Hz}, 1 \mathrm{H}), 7.49(\mathrm{~d}, \mathrm{~J}=3.6 \mathrm{~Hz}, 1 \mathrm{H}), 3.88$ (s, $3 \mathrm{H}), 3.76(\mathrm{~s}, 4 \mathrm{H}), 1.03(\mathrm{~s}, 6 \mathrm{H}) \mathrm{ppm}$.

${ }^{13} \mathrm{C}$ NMR $\left(101 \mathrm{MHz}, \mathrm{CDCl}_{3}\right) \delta 163.3(\mathrm{C}), 135.7(\mathrm{C}), 134.1(\mathrm{CH}), 129.2(\mathrm{CH}), 72.6\left(\mathrm{CH}_{2}\right)$, $52.3\left(\mathrm{O}-\mathrm{CH}_{3}\right), 32.2(\mathrm{C}), 22.0\left(\mathrm{CH}_{3}\right) \mathrm{ppm}$.

GC-MS (El): m/z (relative intensity): 254.1 (40) [M+•], 223.1 (100), 136.9 (25), 111.0 (20), 70.1 (20), 61.0 (20), 43.1 (100).

\section{2da. 1-(5-(4,4,5,5-tetramethyl-1,3,2-dioxaborolan-2-yl)thiophen-2-yl)propan-1-one}<smiles>CCC(=O)c1ccc(B2OC(C)(C)C(C)(C)O2)s1</smiles>

The compound (CAS 2414250-08-9) was prepared according to the general procedure using 1-(5-bromothiophen-2yl)propan-1-one (4.4 mg, $20 \mu \mathrm{mol}, 1.0$ equiv.), bis(pinacolato)diboron (50.6 mg, $200 \mu \mathrm{mol}, 10.0$ equiv.), dodecanenitrile ( $4.4 \mu \mathrm{L}, 20 \mu \mathrm{mol}, 1.0$ equiv.) as internal standard and DIPEA (4.2 $\mu \mathrm{L}, 24$ $\mu \mathrm{mol}, 1.2$ equiv.) and $\mathrm{G} 1$ ( $10 \mathrm{~g} / \mathrm{L})$. The reaction mixture was irradiated for 3 hours, obtaining $74 \%$ product yield according to GC-FID analysis (62\% isolated yield as white powder).

${ }^{1} \mathrm{H}$ NMR $\left(400 \mathrm{MHz}, \mathrm{CDCl}_{3}\right) \delta 7.71(\mathrm{~d}, J=3.7 \mathrm{~Hz}, 1 \mathrm{H}), 7.55(\mathrm{~d}, J=3.7 \mathrm{~Hz}, 1 \mathrm{H}), 2.92(\mathrm{q}$, $J=7.3 \mathrm{~Hz}, 2 \mathrm{H}), 1.32(\mathrm{~s}, 12 \mathrm{H}), 1.21-1.17(\mathrm{t}, 3 \mathrm{H}) \mathrm{ppm}$.

${ }^{13} \mathrm{C}$ NMR (101 MHz, $\left.\mathrm{CDCl}_{3}\right) \delta 193.9(\mathrm{C}), 149.2(\mathrm{C}), 137.3(\mathrm{CH}), 132.0(\mathrm{CH}), 84.7(\mathrm{C})$, $33.2\left(\mathrm{CH}_{2}\right), 24.6\left(\mathrm{CH}_{3}\right), 8.5\left(\mathrm{CH}_{3}\right) \mathrm{ppm}$.

Spectral data were consistent with literature. ${ }^{1}$

2db. 1-(5-(4,4,6-trimethyl-1,3,2-dioxaborinan-2-yl)thiophen-2-yl)propan-1-one<smiles>CCC(=O)c1ccc(B2OC(C)CC(C)(C)O2)s1</smiles>

The compound (CAS 2414250-12-5) was prepared according to the general procedure using 1-(5-bromothiophen-2yl)propan-1-one $\quad(4.4 \mathrm{mg}, 20 \mu \mathrm{mol}, 1.0$ equiv.), bis(hexyleneglycolato)diboron $(53.0 \mathrm{mg}, 200 \mu \mathrm{mol}, 10.0$ equiv.), dodecanenitrile ( $4.4 \mu \mathrm{L}, 20 \mu \mathrm{mol}, 1.0$ equiv.) as internal standard and DIPEA (4.2 $\mu \mathrm{L}, 24 \mu \mathrm{mol}, 1.2$ equiv.) and $\mathrm{G} 1$ (10 g/L). The reaction mixture was irradiated for 3 hours, obtaining $81 \%$ product yield according to GC-FID analysis (69\% isolated yield as white powder).

${ }^{1} \mathrm{H}$ NMR $\left(400 \mathrm{MHz}, \mathrm{CDCl}_{3}\right) \delta 7.68(\mathrm{~d}, J=3.7 \mathrm{~Hz}, 1 \mathrm{H}), 7.48(\mathrm{~d}, J=3.7 \mathrm{~Hz}, 1 \mathrm{H}), 4.33(\mathrm{dqd}$, $J=12.4,6.2,2.9 \mathrm{~Hz}, 1 \mathrm{H}), 2.92(\mathrm{q}, J=7.3 \mathrm{~Hz}, 2 \mathrm{H}), 1.86(\mathrm{dd}, J=14.0,2.9 \mathrm{~Hz}, 1 \mathrm{H}), 1.60$ (dd, $J=13.9,11.7 \mathrm{~Hz}, 1 \mathrm{H}), 1.35(\mathrm{~s}, 6 \mathrm{H}), 1.32(\mathrm{~d}, J=6.2 \mathrm{~Hz}, 3 \mathrm{H}), 1.19(\mathrm{t}, J=7.3 \mathrm{~Hz}, 3 \mathrm{H})$ ppm. 
${ }^{13} \mathrm{C}$ NMR (101 MHz, CDCl $)$ $) 194.1(\mathrm{C}), 148.0(\mathrm{C}), 135.5(\mathrm{CH}), 132.0(\mathrm{CH}), 72.0(\mathrm{C})$, $65.7(\mathrm{CH}), 46.1\left(\mathrm{CH}_{2}\right), 33.1\left(\mathrm{CH}_{2}\right), 31.2\left(\mathrm{CH}_{3}\right), 28.1\left(\mathrm{CH}_{3}\right), 8.6\left(\mathrm{CH}_{3}\right) \mathrm{ppm}$.

Spectral data were consistent with literature. ${ }^{1}$

2dc. 1-(5-(4,4,6,6-tetramethyl-1,3,2-dioxaborinan-2-yl)thiophen-2-yl)propan-1-one<smiles>CCC(=O)c1ccc(B2OC(C)(C)CC(C)(C)O2)s1</smiles>

The compound (CAS 2414250-17-0) was prepared according to the general procedure using 1-(5-bromothiophen-2yl)propan-1-one (4.4 mg, 20 Hmol, 1.0 equiv.), 4,4,4',4',6,6,6',6'octamethyl-2,2'-bi(1,3,2-dioxaborinane) (56.4 mg, $200 \mu \mathrm{mol}$, 10.0 equiv.), dodecanenitrile $(4.4 \mu \mathrm{L}, 20 \mu \mathrm{mol}, 1.0$ equiv.) as internal standard and DIPEA ( $4.2 \mu \mathrm{L}, 24 \mu \mathrm{mol}, 1.2$ equiv.) and $\mathrm{G} 1(10 \mathrm{~g} / \mathrm{L})$. The reaction mixture was irradiated for 3 hours, obtaining $82 \%$ product yield according to GC-FID analysis (67\% isolated yield as white powder).

${ }^{1} \mathrm{H}$ NMR $\left(400 \mathrm{MHz}, \mathrm{CDCl}_{3}\right) \delta 7.71(\mathrm{~d}, J=3.7 \mathrm{~Hz}, 1 \mathrm{H}), 7.51(\mathrm{~d}, J=3.7 \mathrm{~Hz}, 1 \mathrm{H}), 2.94(\mathrm{~d}$, $J=7.3 \mathrm{~Hz}, 2 \mathrm{H}), 1.93(\mathrm{~s}, 2 \mathrm{H}), 1.42(\mathrm{~s}, 12 \mathrm{H}), 1.22(\mathrm{t}, J=7.3 \mathrm{~Hz}, 3 \mathrm{H}) \mathrm{ppm}$.

${ }^{13} \mathrm{C}$ NMR (101 MHz, $\left.\mathrm{CDCl}_{3}\right) \delta 194.2(\mathrm{C}), 147.9(\mathrm{C}), 135.5(\mathrm{CH}), 132.1(\mathrm{CH}), 71.9(\mathrm{C})$, $49.2\left(\mathrm{CH}_{2}\right), 33.2\left(\mathrm{CH}_{3}\right), 8.7\left(\mathrm{CH}_{3}\right) \mathrm{ppm}$.

Spectral data were consistent with literature. ${ }^{1}$

2dd. 1-(5-(5,5-dimethyl-1,3,2-dioxaborinan-2-yl)thiophen-2-yl)propan-1-one<smiles>CCC(=O)c1ccc(B2OCC(C)(C)CO2)s1</smiles>

The compound (CAS 2414250-22-7) was prepared according to the general procedure using 1-(5-bromothiophen-2yl)propan-1-one (4.4 mg, $20 \mu \mathrm{mol}, 1.0$ equiv.), bis(neopentylglycolato)diboron (45.2 $\mathrm{mg}, 200 \mu \mathrm{mol}, 10.0$ equiv.), dodecanenitrile $(4.4 \mu \mathrm{L}, 20 \mu \mathrm{mol}, 1.0$ equiv.) as internal standard and DIPEA $(4.2 \mu \mathrm{L}, 24 \mu \mathrm{mol}, 1.2$ equiv.) and $\mathrm{G} 1(10 \mathrm{~g} / \mathrm{L})$. The reaction mixture was irradiated for 4 hours, obtaining $58 \%$ product yield according to GC-FID analysis ( $43 \%$ isolated yield as white powder).

${ }^{1}$ H NMR $\left(400 \mathrm{MHz}, \mathrm{CDCl}_{3}\right) \delta 7.72(\mathrm{~d}, J=3.7 \mathrm{~Hz}, 1 \mathrm{H}), 7.51(\mathrm{~d}, J=3.7 \mathrm{~Hz}, 1 \mathrm{H}), 3.76(\mathrm{~s}$, 4H), $2.94(\mathrm{q}, J=7.3 \mathrm{~Hz}, 2 \mathrm{H}), 1.22(\mathrm{t}, J=7.3 \mathrm{~Hz}, 3 \mathrm{H}), 1.03(\mathrm{~s}, 6 \mathrm{H}) \mathrm{ppm}$.

${ }^{13} \mathrm{C}$ NMR $\left(101 \mathrm{MHz}, \mathrm{CDCl}_{3}\right) \delta 194.1(\mathrm{C}), 148.4(\mathrm{C}), 135.9(\mathrm{CH}), 132.1(\mathrm{CH}), 72.6\left(\mathrm{CH}_{2}\right)$, $33.2\left(\mathrm{CH}_{2}\right), 32.2(\mathrm{C}), 22.0\left(\mathrm{CH}_{3}\right), 8.6\left(\mathrm{CH}_{3}\right) \mathrm{ppm}$.

Spectral data were consistent with literature. ${ }^{1}$

2ea. 2-methyl-1-(5-(4,4,5,5-tetramethyl-1,3,2-dioxaborolan-2-yl)thiophen-2yl)propan-1-one<smiles>CC(C)C(=O)c1ccc(B2OC(C)(C)C(C)(C)O2)s1</smiles>

The compound (CAS 2414250-09-0) was prepared according to the general procedure using 1-(5-bromothiophen-2-yl)-2methylpropan-1-one (3.2 $\mu \mathrm{L}, 20 \mu \mathrm{mol}, 1.0$ equiv.), bis(pinacolato)diboron (50.6 mg, $200 \mu \mathrm{mol}, 10.0$ equiv.), dodecanenitrile ( $4.4 \mu \mathrm{L}, 20 \mu \mathrm{mol}, 1.0$ equiv.) as internal standard and DIPEA (4.2 $\mu \mathrm{L}, 24$ umol, 1.2 equiv.) and $\mathrm{G} 1$ (10 g/L). The reaction mixture was irradiated for 3 hours, obtaining $77 \%$ product yield according to GC-FID analysis $(61 \%$ isolated yield as white powder).

${ }^{1} \mathrm{H}$ NMR $\left(400 \mathrm{MHz}, \mathrm{CDCl}_{3}\right) \delta 7.75(\mathrm{~d}, J=3.7 \mathrm{~Hz}, 1 \mathrm{H}), 7.58(\mathrm{~d}, J=3.7 \mathrm{~Hz}, 1 \mathrm{H}), 3.36$ (dp, $J=13.7,6.8 \mathrm{~Hz}, 1 \mathrm{H}), 1.34(\mathrm{~s}, 12 \mathrm{H}), 1.23(\mathrm{t}, J=4.4 \mathrm{~Hz}, 3 \mathrm{H}) \mathrm{ppm}$.

${ }^{13} \mathrm{C}$ NMR (101 MHz, $\left.\mathrm{CDCl}_{3}\right) \delta 197.6(\mathrm{C}), 148.7(\mathrm{C}), 137.4(\mathrm{CH}), 132.2(\mathrm{CH}), 84.8(\mathrm{C})$, $38.0(\mathrm{CH}), 24.9\left(\mathrm{CH}_{3}\right), 19.5\left(\mathrm{CH}_{3}\right) \mathrm{ppm}$. 
Spectral data were consistent with literature. ${ }^{1}$

2eb. 2-methyl-1-(5-(4,4,6-trimethyl-1,3,2-dioxaborinan-2-yl)thiophen-2-yl)propan1-one<smiles>CC1CC(C)(C)OB(c2ccc(C(=O)C(C)C)s2)O1</smiles>

The compound (CAS 2414250-13-6) was prepared according to the general procedure using 1-(5-bromothiophen-2-yl)-2methylpropan-1-one (3.2 $\mu \mathrm{L}, 20 \mu \mathrm{mol}, 1.0$ equiv.), bis(hexyleneglycolato)diboron $(53.0 \mathrm{mg}, 200 \mu \mathrm{mol}, 10.0$ equiv.), dodecanenitrile $(4.4 \mu \mathrm{L}, 20 \mu \mathrm{mol}, 1.0$ equiv.) as internal standard and DIPEA ( $4.2 \mu \mathrm{L}, 24 \mu \mathrm{mol}, 1.2$ equiv.) and $\mathrm{G} 1$ (10 g/L). The reaction mixture was irradiated for 3 hours, obtaining $78 \%$ product yield according to GC-FID analysis (66\% isolated yield as white powder).

${ }^{1} \mathrm{H}$ NMR $\left(400 \mathrm{MHz}, \mathrm{CDCl}_{3}\right) \delta 7.67(\mathrm{~d}, J=3.7 \mathrm{~Hz}, 1 \mathrm{H}), 7.46(\mathrm{~d}, J=3.7 \mathrm{~Hz}, 1 \mathrm{H}), 4.30$ (ddd, $J=11.6,6.1,2.9 \mathrm{~Hz}, 1 \mathrm{H}), 3.33(\mathrm{dt}, J=13.7,6.8 \mathrm{~Hz}, 1 \mathrm{H}), 1.83(\mathrm{dd}, J=14.0,2.9 \mathrm{~Hz}, 1 \mathrm{H})$, $1.57(\mathrm{dd}, J=13.9,11.7 \mathrm{~Hz}, 1 \mathrm{H}), 1.32(\mathrm{~s}, 6 \mathrm{H}), 1.29(\mathrm{~d}, J=6.2 \mathrm{~Hz}, 3 \mathrm{H}), 1.17-1.12(\mathrm{~m}$, $6 \mathrm{H}) \mathrm{ppm}$.

${ }^{13} \mathrm{C}$ NMR (101 MHz, $\left.\mathrm{CDCl}_{3}\right) \delta 197.5(\mathrm{C}), 147.3(\mathrm{C}), 135.5(\mathrm{CH}), 132.0(\mathrm{CH}), 71.9(\mathrm{C})$, $65.6(\mathrm{CH}), 46.0\left(\mathrm{CH}_{2}\right), 37.7(\mathrm{CH}), 31.1\left(\mathrm{CH}_{3}\right), 28.1\left(\mathrm{CH}_{3}\right), 23.0\left(\mathrm{CH}_{3}\right), 19.4\left(\mathrm{CH}_{3}\right) \mathrm{ppm}$.

Spectral data were consistent with literature. ${ }^{1}$

2ec. 2-methyl-1-(5-(4,4,6,6-tetramethyl-1,3,2-dioxaborinan-2-yl)thiophen-2yl)propan-1-one<smiles>CC(C)C(=O)c1ccc(B2OC(C)(C)CC(C)(C)O2)s1</smiles>

The compound (CAS 2414250-18-1) was prepared according to the general procedure using 1-(5-bromothiophen-2-yl)-2methylpropan-1-one (3.2 $\mu \mathrm{L}, 20 \mu \mathrm{mol}, 1.0$ equiv.), 4,4,4',4',6,6,6',6'-octamethyl-2,2'-bi(1,3,2-dioxaborinane) (56.4 $\mathrm{mg}, 200 \mu \mathrm{mol}, 10.0$ equiv. $)$, dodecanenitrile $(4.4 \mu \mathrm{L}, 20 \mu \mathrm{mol}$, 1.0 equiv.) as internal standard and DIPEA ( $4.2 \mu \mathrm{L}, 24 \mu \mathrm{mol}, 1.2$ equiv.) and G1 (10 g/L). The reaction mixture was irradiated for 3 hours, obtaining $82 \%$ product yield according to GC-FID analysis ( $66 \%$ isolated yield as white powder).

${ }^{1} \mathrm{H}$ NMR $\left(400 \mathrm{MHz}, \mathrm{CDCl}_{3}\right) \delta 7.72(\mathrm{~d}, J=3.7 \mathrm{~Hz}, 1 \mathrm{H}), 7.52(\mathrm{~d}, J=3.7 \mathrm{~Hz}, 1 \mathrm{H}), 3.38$ (dt, $J=13.7,6.8 \mathrm{~Hz}, 1 \mathrm{H}), 1.93(\mathrm{~s}, 2 \mathrm{H}), 1.42(\mathrm{~s}, 12 \mathrm{H}), 1.23(\mathrm{~d}, J=6.8 \mathrm{~Hz}, 6 \mathrm{H}) \mathrm{ppm}$.

${ }^{13} \mathrm{C}$ NMR $\left(101 \mathrm{MHz}, \mathrm{CDCl}_{3}\right) \delta 197.8(\mathrm{C}), 147.4(\mathrm{C}), 135.6(\mathrm{CH}), 132.2(\mathrm{CH}), 71.9(\mathrm{C})$, $49.2\left(\mathrm{CH}_{2}\right), 37.8(\mathrm{CH}), 31.8\left(\mathrm{CH}_{3}\right), 19.6\left(\mathrm{CH}_{3}\right) \mathrm{ppm}$.

Spectral data were consistent with literature. ${ }^{1}$

2ed. 1-(5-(5,5-dimethyl-1,3,2-dioxaborinan-2-yl)thiophen-2-yl)-2-methylpropan-1one<smiles>CC(C)C(=O)c1ccc(B2OCC(C)(C)CO2)s1</smiles>

The compound (CAS 2414250-23-8) was prepared according to the general procedure using 1-(5-bromothiophen-2-yl)-2methylpropan-1-one (3.2 $\mu \mathrm{L}, 20 \mu \mathrm{mol}, 1.0$ equiv.), bis(neopentylglycolato)diboron (45.2 $\mathrm{mg}, 200 \mu \mathrm{mol}, 10.0$ equiv.), dodecanenitrile $(4.4 \mu \mathrm{L}, 20 \mu \mathrm{mol}, 1.0$ equiv.) as internal standard and DIPEA $(4.2 \mu \mathrm{L}, 24 \mu \mathrm{mol}, 1.2$ equiv. $)$ and $\mathrm{G} 1(10 \mathrm{~g} / \mathrm{L})$. The reaction mixture was irradiated for 2 hours, obtaining 54\% product yield according to GC-FID analysis ( $40 \%$ isolated yield as white powder).

${ }^{1} \mathrm{H}$ NMR $\left(400 \mathrm{MHz}, \mathrm{CDCl}_{3}\right) \delta 7.74(\mathrm{~d}, J=3.7 \mathrm{~Hz}, 1 \mathrm{H}), 7.52(\mathrm{~d}, J=3.7 \mathrm{~Hz}, 1 \mathrm{H}), 3.77(\mathrm{~s}$, $4 \mathrm{H}), 3.43-3.25(\mathrm{~m}, 1 \mathrm{H}), 1.23(\mathrm{~d}, J=6.8 \mathrm{~Hz}, 6 \mathrm{H}), 1.03(\mathrm{~s}, 6 \mathrm{H}) \mathrm{ppm}$. 
${ }^{13} \mathrm{C}$ NMR $\left(101 \mathrm{MHz}, \mathrm{CDCl}_{3}\right) \delta 197.7(\mathrm{C}), 147.8(\mathrm{C}), 136.0(\mathrm{CH}), 132.3(\mathrm{C}), 72.6\left(\mathrm{CH}_{2}\right)$, $37.9(\mathrm{CH}), 32.2(\mathrm{C}), 22.0\left(\mathrm{CH}_{3}\right), 19.5\left(\mathrm{CH}_{3}\right) \mathrm{ppm}$.

Spectral data were consistent with literature. ${ }^{1}$

2 fa.

Phenyl(5-(4,4,5,5-tetramethyl-1,3,2-dioxaborolan-2-yl)thiophen-2yl)methanone<smiles>CC1(C)OB(c2ccc(C(=O)c3ccccc3)s2)OC1(C)C</smiles>

The compound (CAS 1220107-42-5) was prepared according to the general procedure using (5-bromothiophen2-yl)(phenyl)methanone (5.5 mg, $20 \mu \mathrm{mol}, 1.0$ equiv.), bis(pinacolato)diboron (50.6 mg, $200 \mu \mathrm{mol}, 10.0$ equiv.), dodecanenitrile $(4.4 \mu \mathrm{L}, 20 \mu \mathrm{mol}, 1.0$ equiv.) as internal standard and DIPEA (4.2 $\mu \mathrm{L}, 24$ umol, 1.2 equiv.) and $\mathrm{G} 1$ (10 g/L). The reaction mixture was irradiated for 3 hours, obtaining $68 \%$ product yield according to GC-FID analysis ( $51 \%$ isolated yield as yellowpale powder).

${ }^{1}$ H NMR $\left(400 \mathrm{MHz}, \mathrm{CDCl}_{3}\right) \delta 7.91-7.84(\mathrm{~m}, 2 \mathrm{H}), 7.70(\mathrm{~d}, J=3.7 \mathrm{~Hz}, 1 \mathrm{H}), 7.61(\mathrm{~d}, J=$ $3.7 \mathrm{~Hz}, 1 \mathrm{H}), 7.57(\mathrm{~d}, J=7.5 \mathrm{~Hz}, 1 \mathrm{H}), 7.48$ (t, $J=7.5 \mathrm{~Hz}, 2 \mathrm{H}), 1.35$ (s, 12H) ppm.

${ }^{13} \mathrm{C}$ NMR $\left(101 \mathrm{MHz}, \mathrm{CDCl}_{3}\right) \delta 188.8(\mathrm{C}), 148.6(\mathrm{C}), 138.4(\mathrm{C}), 137.1(\mathrm{CH}), 135.0(\mathrm{CH})$, $132.5(\mathrm{CH}), 129.4(2 \times \mathrm{CH}), 128.5(2 \times \mathrm{CH}), 84.8(\mathrm{C}), 24.7\left(\mathrm{CH}_{3}\right) \mathrm{ppm}$.

Spectral data were consistent with literature. ${ }^{1}$

2fb. Phenyl(5-(4,4,6-trimethyl-1,3,2-dioxaborinan-2-yl)thiophen-2-yl)methanone<smiles>CC1CC(C)(C)OB(c2ccc(C(=O)c3ccccc3)s2)O1</smiles>

The compound (CAS 2414250-14-7) was prepared according to the general procedure using (5-bromothiophen2-yl)(phenyl)methanone (5.5 mg, $20 \mu \mathrm{mol}, 1.0$ equiv.), bis(hexyleneglycolato)diboron (53.0 mg, $200 \mu \mathrm{mol}, 10.0$ equiv.), dodecanenitrile $(4.4 \mu \mathrm{L}, 20 \mu \mathrm{mol}, 1.0$ equiv.) as internal standard and DIPEA $(4.2 \mu \mathrm{L}, 24 \mu \mathrm{mol}, 1.2$ equiv.) and $\mathrm{G} 1$ ( $10 \mathrm{~g} / \mathrm{L})$. The reaction mixture was irradiated for 3 hours, obtaining $75 \%$ product yield according to GC-FID analysis (61\% isolated yield as yellow-pale powder).

${ }^{1} \mathrm{H}$ NMR $\left(400 \mathrm{MHz}, \mathrm{CDCl}_{3}\right) \delta 7.77(\mathrm{~d}, J=7.5 \mathrm{~Hz}, 2 \mathrm{H}), 7.57(\mathrm{~d}, J=3.6 \mathrm{~Hz}, 1 \mathrm{H}), 7.49(\mathrm{t}, J$ $=7.1 \mathrm{~Hz}, 1 \mathrm{H}), 7.46-7.43(\mathrm{~m}, 1 \mathrm{H}), 7.39(\mathrm{t}, J=7.5 \mathrm{~Hz}, 2 \mathrm{H}), 4.35-4.21(\mathrm{~m}, 1 \mathrm{H}), 1.81$ (dd, $J=14.0,2.7 \mathrm{~Hz}, 1 \mathrm{H}), 1.59-1.49(\mathrm{~m}, 1 \mathrm{H}), 1.29(\mathrm{~s}, 6 \mathrm{H}), 1.26(\mathrm{~d}, J=6.2 \mathrm{~Hz}, 3 \mathrm{H})$ ppm.

${ }^{13} \mathrm{C}$ NMR $\left(101 \mathrm{MHz}, \mathrm{CDCl}_{3}\right) \delta 188.0(\mathrm{C}), 147.1(\mathrm{C}), 138.3(\mathrm{CH}), 135.2(\mathrm{CH}), 134.8(\mathrm{CH})$, $132.1(\mathrm{CH}), 129.1(2 \times \mathrm{CH}), 128.2(2 \times \mathrm{CH}), 71.8(\mathrm{C}), 65.5(\mathrm{CH}), 45.6\left(\mathrm{CH}_{2}\right), 31.0\left(\mathrm{CH}_{3}\right)$, $27.6\left(\mathrm{CH}_{3}\right), 22.9\left(\mathrm{CH}_{3}\right) \mathrm{ppm}$.

Spectral data were consistent with literature. ${ }^{1}$

$2 \mathrm{fc}$.

Phenyl(5-(4,4,6,6-tetramethyl-1,3,2-dioxaborinan-2-yl)thiophen-2yl)methanone<smiles>CC1(C)CC(C)(C)OB(c2ccc(C(=O)c3ccccc3)s2)O1</smiles>

The compound (CAS 2414250-19-2) was prepared according to the general procedure using (5-bromothiophen2-yl)(phenyl)methanone (5.5 mg, $20 \mu \mathrm{mol}, 1.0$ equiv.), $4,4,4^{\prime}, 4$ ', 6, 6, 6',6'-octamethyl-2,2'-bi(1,3,2-dioxaborinane) (56.4 mg, $200 \mu \mathrm{mol}, 10.0$ equiv.), dodecanenitrile $(4.4 \mu \mathrm{L}, 20$ $\mu \mathrm{mol}, 1.0$ equiv.) as internal standard and DIPEA (4.2 $\mu \mathrm{L}, 24 \mu \mathrm{mol}, 1.2$ equiv.) and $\mathrm{G} 1$ $(10 \mathrm{~g} / \mathrm{L})$. The reaction mixture was irradiated for 3 hours, obtaining $81 \%$ product yield according to GC-FID analysis ( $70 \%$ isolated yield as yellow-pale powder). 
${ }^{1} \mathrm{H}$ NMR $\left(400 \mathrm{MHz}, \mathrm{CDCl}_{3}\right) \delta 7.91-7.84(\mathrm{~m}, 2 \mathrm{H}), 7.67(\mathrm{~d}, J=3.7 \mathrm{~Hz}, 1 \mathrm{H}), 7.57$ (dd, $J=$ $12.3,5.5 \mathrm{~Hz}, 2 \mathrm{H}), 7.48(\mathrm{t}, J=7.5 \mathrm{~Hz}, 2 \mathrm{H}), 1.94(\mathrm{~s}, 2 \mathrm{H}), 1.43(\mathrm{~s}, 12 \mathrm{H}) \mathrm{ppm}$.

${ }^{13} \mathrm{C}$ NMR $\left(101 \mathrm{MHz}, \mathrm{CDCl}_{3}\right) \delta 188.5(\mathrm{C}), 147.3(\mathrm{C}), 138.7(\mathrm{C}), 135.3(\mathrm{CH}), 132.3(\mathrm{CH})$, $129.4(2 \times \mathrm{CH}), 128.5(2 \times \mathrm{CH}), 71.9(\mathrm{C}), 49.2\left(\mathrm{CH}_{2}\right), 31.8\left(\mathrm{CH}_{3}\right) \mathrm{ppm}$.

Spectral data were consistent with literature. ${ }^{1}$

2fd. (5-(5,5-dimethyl-1,3,2-dioxaborinan-2-yl)thiophen-2-yl)(phenyl)methanone<smiles>CC1(C)COB(c2ccc(C(=O)c3ccccc3)s2)OC1</smiles>

The compound (CAS 2414250-24-9) was prepared according to the general procedure using (5bromothiophen-2-yl)(phenyl)methanone $(5.5 \mathrm{mg}, 20 \mu \mathrm{mol}$, 1.0 equiv.), bis(neopentylglycoltao)diboron $(45.2 \mathrm{mg}, 200$ $\mu \mathrm{mol}, 10.0$ equiv.), dodecanenitrile $(4.4 \mu \mathrm{L}, 20 \mu \mathrm{mol}, 1.0$ equiv.) as internal standard and DIPEA (4.2 $\mu \mathrm{L}, 24 \mu \mathrm{mol}, 1.2$ equiv.) and $\mathrm{G} 1(10 \mathrm{~g} / \mathrm{L})$. The reaction mixture was irradiated for 2 hours, obtaining $42 \%$ product yield according to GC-FID analysis (35\% isolated yield as yellow-pale powder).

${ }^{1}$ H NMR (400 MHz, CDCl3) $\delta 7.88$ (d, J = 7.4 Hz, 2H), 7.69 (d, J = 3.7 Hz, 1H), 7.57 (dd, $\mathrm{J}=12.0,5.5 \mathrm{~Hz}, 2 \mathrm{H}), 7.48(\mathrm{t}, \mathrm{J}=7.6 \mathrm{~Hz}, 2 \mathrm{H}), 3.78(\mathrm{~s}, 4 \mathrm{H}), 1.04(\mathrm{~s}, 6 \mathrm{H}) \mathrm{ppm}$.

${ }^{13} \mathrm{C}$ NMR $\left(101 \mathrm{MHz}, \mathrm{CDCl}_{3}\right) \delta 188.4(\mathrm{C}), 147.7(\mathrm{C}), 138.5(\mathrm{C}), 135.7(\mathrm{CH}), 135.2(\mathrm{CH})$, $132.4(\mathrm{CH}), 129.4(\mathrm{CH}), 128.5(\mathrm{CH}), 72.7\left(\mathrm{CH}_{2}\right), 32.2(\mathrm{C}), 22.0\left(\mathrm{CH}_{3}\right) \mathrm{ppm}$.

Spectral data were consistent with literature. ${ }^{1}$

\section{FURANS}

3aa. 1-(5-(4,4,5,5-tetramethyl-1,3,2-dioxaborolan-2-yl)furan-2-yl)ethanone<smiles>CC(=O)c1ccc(B2OC(C)(C)C(C)(C)O2)o1</smiles>

The compound was prepared according to the general procedure using 1-(5-bromofuran-2-yl)ethanone (3.8 mg, $20 \mu \mathrm{mol}, 1.0$ equiv.), bis(pinacolato)diboron (50.6 mg, $200 \mu \mathrm{mol}, 10.0$ equiv.), dodecanenitrile $(4.4 \mu \mathrm{L}, 20 \mu \mathrm{mol}, 1.0$ equiv. $)$ as internal standard and DIPEA (4.2 $\mu \mathrm{L}, 24 \mu \mathrm{mol}, 1.2$ equiv.) and $\mathrm{G} 1$ (10 g/L). The reaction mixture was irradiated for 22 hours, obtaining $56 \%$ product yield according to GC-FID analysis (45\% isolated yield as yellow-pale powder).

${ }^{1} \mathrm{H}$ NMR $\left(400 \mathrm{MHz}, \mathrm{CDCl}_{3}\right) \delta 7.17(\mathrm{~d}, \mathrm{~J}=3.5 \mathrm{~Hz}, 1 \mathrm{H}), 7.09(\mathrm{~d}, \mathrm{~J}=3.5 \mathrm{~Hz}, 1 \mathrm{H}), 2.54$ (s, $3 \mathrm{H}), 1.36(\mathrm{~s}, \mathrm{~J}=5.3 \mathrm{~Hz}, 12 \mathrm{H}) \mathrm{ppm}$.

${ }^{13} \mathrm{C}$ NMR (101 MHz, $\left.\mathrm{CDCl}_{3}\right) \delta 188.6(\mathrm{C}), 163.2(\mathrm{C}), 124.5(\mathrm{CH}), 116.5(\mathrm{CH}), 84.9(\mathrm{C})$, $26.6\left(\mathrm{CH}_{3}\right), 24.8\left(\mathrm{CH}_{3}\right) \mathrm{ppm}$.

HRMS (El): $\mathrm{m} / \mathrm{z}(\mathrm{M}+\mathrm{H})^{+}=$calcd. for $\mathrm{C}_{12} \mathrm{H}_{18} \mathrm{BO}_{4}: 237.1293$, found: 237.1297 .

\section{3ab. 1-(5-(4,4,6-trimethyl-1,3,2-dioxaborinan-2-yl)furan-2-yl)ethanone}<smiles>CC(=O)c1ccc(B2OC(C)CC(C)(C)O2)o1</smiles>

The compound was prepared according to the general procedure using 1-(5-bromofuran-2-yl)ethenone (3.8 mg, $80 \mu \mathrm{mol}, 1.0$ equiv.), bis(hexyleneglycolato)diboron ( $53.0 \mathrm{mg}, 200 \mu \mathrm{mol}, 10.0$ equiv.), dodecanenitrile ( $4.4 \mu \mathrm{L}, 20 \mu \mathrm{mol}, 1.0$ equiv.) as internal standard and DIPEA ( $4.2 \mu \mathrm{L}, 24 \mu \mathrm{mol}, 1.2$ equiv.) and G1 (10 g/L). The reaction mixture was irradiated for 6 hours, obtaining $85 \%$ product yield according to GC-FID analysis (63\% isolated yield as white powder).

${ }^{1} \mathrm{H}$ NMR $\left(400 \mathrm{MHz}, \mathrm{CDCl}_{3}\right) \delta 7.14(\mathrm{~d}, \mathrm{~J}=3.5 \mathrm{~Hz}, 1 \mathrm{H}), 6.97$ (d, J = 3.5 Hz, $\left.1 \mathrm{H}\right), 4.36$ (ddd, $\mathrm{J}=11.6,6.1,2.9 \mathrm{~Hz}, 1 \mathrm{H}), 2.52(\mathrm{~s}, 3 \mathrm{H}), 1.88(\mathrm{dd}, \mathrm{J}=14.1,2.9 \mathrm{~Hz}, 1 \mathrm{H}), 1.65-1.59(\mathrm{~m}$, $1 \mathrm{H}), 1.37(\mathrm{~d}, \mathrm{~J}=2.4 \mathrm{~Hz}, 6 \mathrm{H}), 1.35(\mathrm{~d}, \mathrm{~J}=6.2 \mathrm{~Hz}, 3 \mathrm{H}) \mathrm{ppm}$. 
${ }^{13} \mathrm{C}$ NMR (101 MHz, $\left.\mathrm{CDCl}_{3}\right) \delta 187.8(\mathrm{C}), 155.8(\mathrm{C}), 122.6(\mathrm{CH}), 117.0(\mathrm{CH}), 72.2(\mathrm{C})$, $65.8(\mathrm{CH}), 46.2\left(\mathrm{CH}_{2}\right), 31.2\left(\mathrm{CH}_{3}\right), 28.2\left(\mathrm{CH}_{3}\right), 23.1\left(\mathrm{CH}_{3}\right) \mathrm{ppm}$.

HRMS (EI): $\mathrm{m} / \mathrm{z}(\mathrm{M}+\mathrm{H})^{+}=$calcd. for $\mathrm{C}_{12} \mathrm{H}_{17} \mathrm{BO}_{4}: 236.0706$, found: 236.0711 .

\section{3ac. 1-(5-(4,4,6,6-tetramethyl-1,3,2-dioxaborinan-2-yl)furan-2-yl)ethanone}<smiles>CC(=O)c1ccc(B2OC(C)(C)CC(C)(C)O2)o1</smiles>

The compound was prepared according to the general procedure using 1-(5-bromofuran-2-yl)ethanone $(3.8 \mathrm{mg}, 80 \mu \mathrm{mol}, 1.0$ equiv.), 4,4,4',4',6,6,6',6'-octamethyl-2,2'-bi(1,3,2-dioxaborinane) (56.4 mg, $200 \mu \mathrm{mol}, 10.0$ equiv.), dodecanenitrile $(4.4 \mu \mathrm{L}, 20$ $\mu \mathrm{mol}, 1.0$ equiv.) as internal standard and DIPEA $(4.2 \mu \mathrm{L}, 24 \mu \mathrm{mol}$, 1.2 equiv.) and $\mathrm{G} 1$ (10 g/L). The reaction mixture was irradiated for 6 hours, obtaining $73 \%$ product yield according to GC-FID analysis $(60 \%$ isolated yield as yellow-pale powder).

${ }^{1} \mathrm{H}$ NMR $\left(400 \mathrm{MHz}, \mathrm{CDCl}_{3}\right) \delta 7.10(\mathrm{~d}, \mathrm{~J}=3.5 \mathrm{~Hz}, 1 \mathrm{H}), 6.93(\mathrm{~d}, \mathrm{~J}=3.5 \mathrm{~Hz}, 1 \mathrm{H}), 2.46(\mathrm{~s}$, $3 \mathrm{H}), 1.88(\mathrm{~s}, 2 \mathrm{H}), 1.37(\mathrm{~s}, 12 \mathrm{H}) \mathrm{ppm}$.

${ }^{13} \mathrm{C}$ NMR $\left(101 \mathrm{MHz}, \mathrm{CDCl}_{3}\right) \delta 187.7(\mathrm{C}), 155.6(\mathrm{C}), 122.4(\mathrm{CH}), 117.0(\mathrm{CH}), 71.8(\mathrm{C})$, $49.2\left(\mathrm{CH}_{2}\right), 31.6\left(\mathrm{CH}_{3}\right), 26.3\left(\mathrm{CH}_{3}\right) \mathrm{ppm}$.

GC-MS (El): m/z (relative intensity): 250.1 (20) [M+•], 235.1 (30), 251.1 (30), 151.1 (20), 137.0 (20), 70.1 (20), 61.1 (20), 56.1 (10), 43.1 (100).

\section{3ad. 1-(5-(5,5-dimethyl-1,3,2-dioxaborinan-2-yl)furan-2-yl)ethanone}<smiles>CC(=O)c1ccc(B2OCC(C)(C)CO2)o1</smiles>

The compound was prepared according to the general procedure using 1-(5-bromofuran-2-yl)ethanone $(3.8 \mathrm{mg}, 80 \mu \mathrm{mol}, 1.0$ equiv.), bis(neopentylglycoltao)diboron $(45.2 \mathrm{mg}, 200 \mu \mathrm{mol}, 10.0$ equiv.), dodecanenitrile ( $4.4 \mu \mathrm{L}, 20 \mu \mathrm{mol}, 1.0$ equiv.) as internal standard and DIPEA (4.2 $\mu \mathrm{L}, 24 \mu \mathrm{mol}, 1.2$ equiv.) and $\mathrm{G} 1$ (10 $\mathrm{g} / \mathrm{L})$. The reaction mixture was irradiated for 20 hours, obtaining $51 \%$ product yield according to GC-FID analysis (39\% isolated yield as yellow-pale powder).

${ }^{1} \mathrm{H}$ NMR $\left(400 \mathrm{MHz}, \mathrm{CDCl}_{3}\right) \delta 7.15(\mathrm{~d}, \mathrm{~J}=3.5 \mathrm{~Hz}, 1 \mathrm{H}), 7.00(\mathrm{~d}, \mathrm{~J}=3.5 \mathrm{~Hz}, 1 \mathrm{H}), 3.77(\mathrm{~s}$, $4 \mathrm{H}), 2.53(\mathrm{~s}, 3 \mathrm{H}), 1.03(\mathrm{~s}, 6 \mathrm{H}) \mathrm{ppm}$.

${ }^{13} \mathrm{C}$ NMR (101 MHz, $\left.\mathrm{CDCl}_{3}\right) \delta 187.7(\mathrm{C}), 156.0(\mathrm{C}), 123.0(\mathrm{CH}), 116.8(\mathrm{CH}), 72.5(\mathrm{C})$, $32.1\left(\mathrm{CH}_{2}\right), 26.5\left(\mathrm{CH}_{3}\right), 21.4\left(\mathrm{CH}_{3}\right) \mathrm{ppm}$.

HRMS (EI): $\mathrm{m} / \mathrm{z}(\mathrm{M}+\mathrm{H})^{+}=$calcd. for $\mathrm{C}_{11} \mathrm{H}_{15} \mathrm{BO}_{4}: 223.1117$, found: 223.1125 .

\section{1-(5-(phenylthio)furan-2-yl)ethanone}

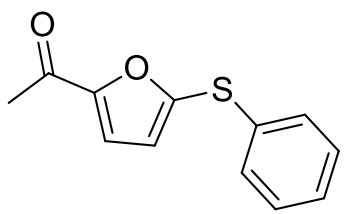

The compound (CAS: 28569-36-0) was prepared according to the general procedure using 1-(5-bromofuran-2-yl)ethanone $(3.8 \mathrm{mg}$, $80 \mu \mathrm{mol}, 1.0$ equiv.), bis(pinacolato)diboron (50.6 mg, $200 \mu \mathrm{mol}$, 10.0 equiv.), diphenyldisulfide ( $4.4 \mathrm{mg}, 80 \mu \mathrm{mol}, 1.0$ equiv.) dodecanenitrile ( $4.4 \mu \mathrm{L}, 20 \mu \mathrm{mol}, 1.0$ equiv.) as internal standard and DIPEA ( $4.2 \mu \mathrm{L}, 24 \mu \mathrm{mol}, 1.2$ equiv.) and $\mathrm{G} 1$ (10 $\mathrm{g} / \mathrm{L})$. The reaction mixture was irradiated for 3 hours, obtaining $78 \%$ product yield according to GC-FID analysis (54\% isolated yield as brown-yellow powder).

This compound is commercially available, and the spectral data were consistent with the commercial one. Please find this compound in Merck:

https://www.sigmaaldrich.com/catalog/product/aldrich/t119652?lang=es\&region=ES 
${ }^{1} \mathrm{H}$ NMR $\left(400 \mathrm{MHz}, \mathrm{CDCl}_{3}\right) \delta 7.31(\mathrm{~m}, 5 \mathrm{H}), 7.17(\mathrm{~d}, \mathrm{~J}=3.5 \mathrm{~Hz}, 1 \mathrm{H}), 6.63(\mathrm{~d}, \mathrm{~J}=3.5 \mathrm{~Hz}$, $1 \mathrm{H}), 2.45(\mathrm{~s}, 3 \mathrm{H}) \mathrm{ppm}$.

${ }^{13} \mathrm{C}$ NMR (101 MHz, CDCl $)$ $\delta 186.5$ (C), $155.1(\mathrm{C}), 150.6$ (C), $133.2(\mathrm{C}), 130.3(\mathrm{CH})$, $129.6(\mathrm{CH}), 127.9(\mathrm{CH}), 118.6(\mathrm{CH}), 118.3(\mathrm{CH}), 26.1\left(\mathrm{CH}_{3}\right) \mathrm{ppm}$.

\section{3ba. 5-(4,4,5,5-tetramethyl-1,3,2-dioxaborolan-2-yl)furan-2-carbaldehyde}<smiles>CC1(C)OB(c2ccc(C=O)o2)OC1(C)C</smiles>

The compound (CAS 273731-82-1) was prepared according to the general procedure using 5-bromofuran-2-carbaldehyde (3.6 $\mathrm{mg}, 20 \mu \mathrm{mol}, 1.0$ equiv.), bis(pinacolato)diboron (50.6 mg, 200 $\mu \mathrm{mol}, 10.0$ equiv.), dodecanenitrile ( $4.4 \mu \mathrm{L}, 20 \mu \mathrm{mol}, 1.0$ equiv.) as internal standard and DIPEA $(4.2 \mu \mathrm{L}, 24 \mu \mathrm{mol}, 1.2$ equiv. $)$ and G1 (10 g/L). The reaction mixture was irradiated for 5 hours, obtaining 35\% product yield according to GC-FID analysis (23\% isolated yield as yellow-pale powder).

Spectral data were consistent with literature. ${ }^{3}$

${ }^{1} \mathrm{H}$ NMR $\left(400 \mathrm{MHz}, \mathrm{CDCl}_{3}\right) \delta 9.80(\mathrm{~s}, 1 \mathrm{H}), 7.23(\mathrm{~d}, \mathrm{~J}=3.6 \mathrm{~Hz}, 1 \mathrm{H}), 7.13(\mathrm{~d}, \mathrm{~J}=3.6 \mathrm{~Hz}$, $1 \mathrm{H}), 1.35(\mathrm{~s}, 12 \mathrm{H}) \mathrm{ppm}$.

${ }^{13} \mathrm{C}$ NMR (101 MHz, $\left.\mathrm{CDCl}_{3}\right) \delta 179.4(\mathrm{C}), 156.4(\mathrm{C}), 124.5(\mathrm{CH}), 118.5(\mathrm{CH}), 85.1(\mathrm{C})$, $24.9\left(\mathrm{CH}_{3}\right) \mathrm{ppm}$.

\section{3bb. 5-(4,4,6-trimethyl-1,3,2-dioxaborinan-2-yl)furan-2-carbaldehyde}<smiles>CC1CC(C)(C)OB(c2ccc(C=O)o2)O1</smiles>

The compound (CAS 1603976-11-9) was prepared according to the general procedure using methyl 5-bromo-2-furaldehyde (3.6 $\mathrm{mg}, 80 \mu \mathrm{mol}, 1.0$ equiv.), bis(hexyleneglycolato)diboron (53.0 $\mathrm{mg}, 200 \mu \mathrm{mol}, 10.0$ equiv.), dodecanenitrile $(4.4 \mu \mathrm{L}, 20 \mu \mathrm{mol}, 1.0$ equiv.) as internal standard and DIPEA ( $4.2 \mu \mathrm{L}, 24 \mu \mathrm{mol}, 1.2$ equiv.) and G1 (10 g/L). The reaction mixture was irradiated for 20 hours, obtaining $54 \%$ product yield according to GC-FID analysis (45\% isolated yield as yellow-pale powder).

${ }^{1} \mathrm{H}$ NMR $\left(400 \mathrm{MHz}, \mathrm{CDCl}_{3}\right) \delta 9.77(\mathrm{~s}, 1 \mathrm{H}), 7.21(\mathrm{~d}, \mathrm{~J}=3.6 \mathrm{~Hz}, 1 \mathrm{H}), 7.03(\mathrm{~d}, \mathrm{~J}=3.5 \mathrm{~Hz}$, $1 \mathrm{H}), 4.37(\mathrm{~m}, \mathrm{~J}=12.3,6.2,2.8 \mathrm{~Hz}, 1 \mathrm{H}), 1.90(\mathrm{dd}, \mathrm{J}=14.1,2.9 \mathrm{~Hz}, 1 \mathrm{H}), 1.65(\mathrm{~d}, \mathrm{~J}=12.0$ $\mathrm{Hz}, 1 \mathrm{H}), 1.39(\mathrm{~d}, \mathrm{~J}=2.9 \mathrm{~Hz}, 6 \mathrm{H}), 1.36(\mathrm{~d}, \mathrm{~J}=6.2 \mathrm{~Hz}, 3 \mathrm{H}) \mathrm{ppm}$.

${ }^{13} \mathrm{C}$ NMR (101 MHz, CDCl $)$ $\delta 179.4(\mathrm{C}), 155.8(\mathrm{C}), 122.6 .1(\mathrm{CH}), 119.5(\mathrm{CH}), 72.4(\mathrm{C})$, $65.9(\mathrm{CH}), 46.3\left(\mathrm{CH}_{2}\right), 31.2\left(\mathrm{CH}_{3}\right), 28.2\left(\mathrm{CH}_{3}\right), 23.1\left(\mathrm{CH}_{3}\right) \mathrm{ppm}$.

HRMS (El): $\mathrm{m} / \mathrm{z}(\mathrm{M}+\mathrm{H})^{+}=$calcd. for $\mathrm{C}_{11} \mathrm{H}_{16} \mathrm{BO}_{4}: 223.1136$, found: 223.1139 .

\section{3bc. 5-(4,4,6,6-tetramethyl-1,3,2-dioxaborinan-2-yl)furan-2-carbaldehyde}<smiles>CC1(C)CC(C)(C)OB(c2ccc(C=O)o2)O1</smiles>

The compound was prepared according to the general procedure using methyl 5-bromo-2-furaldehyde (3.6 mg, $80 \mu \mathrm{mol}, 1.0$ equiv.), 4,4,4',4',6,6,6',6'-octamethyl-2,2'-bi(1,3,2-dioxaborinane) (56.4 mg, $200 \mu \mathrm{mol}, 10.0$ equiv.), dodecanenitrile $(4.4 \mu \mathrm{L}, 20$ $\mu \mathrm{mol}, 1.0$ equiv.) as internal standard and DIPEA $(4.2 \mu \mathrm{L}, 24$ $\mu$ mol, 1.2 equiv.) and $\mathrm{G} 1$ (10 g/L). The reaction mixture was irradiated for 20 hours, obtaining $66 \%$ product yield according to GC-FID analysis (58\% isolated yield yellowpale powder).

${ }^{1}$ H NMR $\left(400 \mathrm{MHz}, \mathrm{CDCl}_{3}\right) \delta 9.77(\mathrm{~s}, 1 \mathrm{H}), 7.22(\mathrm{~d}, \mathrm{~J}=3.5 \mathrm{~Hz}, 1 \mathrm{H}), 7.04(\mathrm{~d}, \mathrm{~J}=3.5 \mathrm{~Hz}$, $1 \mathrm{H}), 1.95(\mathrm{~s}, 2 \mathrm{H}), 1.44(\mathrm{~s}, 12 \mathrm{H}) \mathrm{ppm}$.

\footnotetext{
${ }^{3}$ Akitake Yamaguchi, Sherif J. Kaldas, Solomon D. Appavoo, Diego B. Diaz and Andrei K. Yudi. Chem. Commun., 2019, 55, 10567-10570.
} 
${ }^{13} \mathrm{C}$ NMR (101 MHz, $\left.\mathrm{CDCl}_{3}\right) \delta 179.4(\mathrm{C}), 155.8(\mathrm{C}), 126.7(\mathrm{CH}), 119.6(\mathrm{CH}), 72.1(\mathrm{C})$, $49.3\left(\mathrm{CH}_{2}\right), 31.8\left(\mathrm{CH}_{3}\right) \mathrm{ppm}$.

HRMS (EI): $\mathrm{m} / \mathrm{z}(\mathrm{M}+\mathrm{H})^{+}=$calcd. for $\mathrm{C}_{12} \mathrm{H}_{17} \mathrm{BO}_{4}: 237.1293$, found: 237.1299 .

\section{3bd. 5-(5,5-dimethyl-1,3,2-dioxaborinan-2-yl)furan-2-carbaldehyde}<smiles>CC1(C)COB(c2ccc(C=O)o2)OC1</smiles>

The compound (CAS 1218791-07-1) was prepared according to the general procedure using 5-bromofuran-2-carbaldehyde (3.6 $\mathrm{mg}, 80 \mu \mathrm{mol}, 1.0$ equiv.), bis(neopentylglycoltao)diboron (45.2 $\mathrm{mg}, 200 \mu \mathrm{mol}, 10.0$ equiv.), dodecanenitrile $(4.4 \mu \mathrm{L}, 20 \mu \mathrm{mol}$, 1.0 equiv.) as internal standard and DIPEA $(4.2 \mu \mathrm{L}, 24 \mu \mathrm{mol}, 1.2$ equiv.) and $\mathrm{G} 1(10 \mathrm{~g} / \mathrm{L})$. The reaction mixture was irradiated for 20 hours, obtaining $80 \%$ product yield according to GC-FID analysis (62\% isolated yield as yellow-pale powder).

${ }^{1} \mathrm{H}$ NMR $\left(400 \mathrm{MHz}, \mathrm{CDCl}_{3}\right) \delta 9.78(\mathrm{~s}, 1 \mathrm{H}), 7.22(\mathrm{~d}, \mathrm{~J}=3.6 \mathrm{~Hz}, 1 \mathrm{H}), 7.05(\mathrm{~d}, \mathrm{~J}=3.6 \mathrm{~Hz}$, $1 \mathrm{H}), 3.78(\mathrm{~s}, 4 \mathrm{H}), 1.03(\mathrm{~s}, 6 \mathrm{H}) \mathrm{ppm}$.

${ }^{13} \mathrm{C}$ NMR (101 MHz, CDCl $\left.{ }_{3}\right) \delta 179.2(\mathrm{C}), 156.0(\mathrm{C}), 123.0(\mathrm{CH}), 119.0(\mathrm{CH}), 72.6(\mathrm{C})$, $32.3\left(\mathrm{CH}_{2}\right), 22.0\left(\mathrm{CH}_{3}\right) \mathrm{ppm}$.

HRMS (EI): $\mathrm{m} / \mathrm{z}(\mathrm{M}+\mathrm{H})^{+}=$calcd. for $\mathrm{C}_{10} \mathrm{H}_{14} \mathrm{BO}_{4}: 209.0985$, found: 209.0982 .

\section{3ca. Methyl 5-(4,4,5,5-tetramethyl-1,3,2-dioxaborolan-2-yl)furan-2-carboxylate}<smiles>COC(=O)c1ccc(B2OC(C)(C)C(C)(C)O2)o1</smiles>

The compound (CAS 676501-87-4) was prepared according to the general procedure using methyl 5-bromofuran-2carboxylate $(4.1 \quad \mathrm{mg}, 20 \mu \mathrm{mol}, 1.0 \quad$ equiv. $)$, bis(pinacolato)diboron (50.6 mg, $200 \mu \mathrm{mol}, 10.0$ equiv.), dodecanenitrile $(4.4 \mu \mathrm{L}, 20 \mu \mathrm{mol}, 1.0$ equiv.) as internal standard and DIPEA (4.2 $\mu \mathrm{L}, 24 \mu \mathrm{mol}, 1.2$ equiv.) and $\mathrm{G} 1(10 \mathrm{~g} / \mathrm{L})$. The reaction mixture was irradiated for 22 hours, obtaining $60 \%$ product yield according to GC-FID analysis ( $51 \%$ isolated yield as yellow-pale powder).

Spectral data were consistent with literature. ${ }^{4}$

${ }^{1} \mathrm{H}$ NMR $\left(400 \mathrm{MHz}, \mathrm{CDCl}_{3}\right) \delta 7.16(\mathrm{dd}, \mathrm{J}=13.5,3.5 \mathrm{~Hz}, 1 \mathrm{H}), 7.07(\mathrm{~d}, \mathrm{~J}=3.5 \mathrm{~Hz}, 1 \mathrm{H})$, $3.88(\mathrm{~s}, 3 \mathrm{H}), 1.33(\mathrm{~s}, 12 \mathrm{H}) \mathrm{ppm}$.

${ }^{13} \mathrm{C}$ NMR (101 MHz, CDCl $\left.{ }_{3}\right) \delta 159.3$ (C), 148.5 (C), 124.3 (C), 118.1 (C), 84.9 (C), 52.1 $\left(\mathrm{O}-\mathrm{CH}_{3}\right), 24.2\left(\mathrm{CH}_{3}\right) \mathrm{ppm}$.

\section{3cb. Methyl 5-(4,4,6-trimethyl-1,3,2-dioxaborinan-2-yl)furan-2-carboxylate}<smiles>COC(=O)c1ccc(B2OC(C)CC(C)(C)O2)o1</smiles>

The compound (CAS 1451144-93-6) was prepared according to the general procedure using methyl 5-bromofuran-2carboxylate $(4.1 \quad \mathrm{mg}, \quad 80 \mu \mathrm{mol}, \quad 1.0 \quad$ equiv. $)$, bis(hexyleneglycolato)diboron $(53.0 \mathrm{mg}, 200 \mu \mathrm{mol}, 10.0$ equiv.), dodecanenitrile $(4.4 \mu \mathrm{L}, 20 \mu \mathrm{mol}, 1.0$ equiv.) as internal standard and DIPEA $(4.2 \mu \mathrm{L}, 24 \mu \mathrm{mol}, 1.2$ equiv.) and $\mathrm{G} 1$

$(10 \mathrm{~g} / \mathrm{L})$. The reaction mixture was irradiated for 20 hours, obtaining $74 \%$ product yield according to GC-FID analysis ( $61 \%$ isolated yield as white powder).

Spectral data were consistent with literature. ${ }^{5}$

${ }^{1} \mathrm{H}$ NMR $\left(400 \mathrm{MHz}, \mathrm{CDCl}_{3}\right) \delta 7.11(\mathrm{~d}, \mathrm{~J}=3.4 \mathrm{~Hz}, 1 \mathrm{H}), 6.92(\mathrm{~d}, \mathrm{~J}=3.4 \mathrm{~Hz}, 1 \mathrm{H}), 4.31$ (dtd, $\mathrm{J}=12.4,6.2,2.9 \mathrm{~Hz}, 1 \mathrm{H}), 3.85(\mathrm{~s}, 3 \mathrm{H}), 1.84(\mathrm{dd}, \mathrm{J}=14.0,2.9 \mathrm{~Hz}, 1 \mathrm{H}), 1.63-1.52(\mathrm{~m}$, $1 \mathrm{H}), 1.33(\mathrm{~d}, \mathrm{~J}=3.8 \mathrm{~Hz}, 6 \mathrm{H}), 1.31(\mathrm{~d}, \mathrm{~J}=6.2 \mathrm{~Hz}, 3 \mathrm{H}) \mathrm{ppm}$.

\footnotetext{
${ }^{4}$ Neely, Jamie M.; Bezdek, Mate J.; Chirik, Paul J. ACS Central Science 2016, 2, (12), 935-942.

5 Liskey, Carl W.; Hartwig, John F. Synthesis 2013, 45(13), 1837-1842.
} 
${ }^{13} \mathrm{C}$ NMR (101 MHz, $\left.\mathrm{CDCl}_{3}\right) \delta 159.4(\mathrm{C}), 147.5(\mathrm{C}), 122.0(\mathrm{C}), 118.0(\mathrm{CH}), 116.4(\mathrm{CH})$, $72.0(\mathrm{C}), 65.7(\mathrm{CH}), 51.9\left(\mathrm{O}-\mathrm{CH}_{3}\right), 46.2\left(\mathrm{CH}_{2}\right), 31.0\left(\mathrm{CH}_{3}\right), 28.1\left(\mathrm{CH}_{3}\right), 23.0\left(\mathrm{CH}_{3}\right) \mathrm{ppm}$.

\section{3cc. Methyl 5-(4,4,6,6-tetramethyl-1,3,2-dioxaborinan-2-yl)furan-2-carboxylate}<smiles>COC(=O)c1ccc(B2OC(C)(C)CC(C)(C)O2)o1</smiles>

The compound was prepared according to the general procedure using methyl 5-bromofuran-2-carboxylate $(4.1 \mathrm{mg}$, $80 \mu \mathrm{mol}, \quad 1.0$ equiv.), 4,4,4',4',6,6,6',6'-octamethyl-2,2'bi(1,3,2-dioxaborinane) (56.4 mg, $200 \mu \mathrm{mol}, 10.0$ equiv.), dodecanenitrile $(4.4 \mu \mathrm{L}, 20 \mu \mathrm{mol}, 1.0$ equiv.) as internal standard and DIPEA ( $4.2 \mu \mathrm{L}, 24 \mu \mathrm{mol}, 1.2$ equiv.) and G1 (10 g/L). The reaction mixture was irradiated for 20 hours, obtaining $75 \%$ product yield according to GC-FID analysis (64\% isolated yield as white powder).

${ }^{1} \mathrm{H}$ NMR $\left(400 \mathrm{MHz}, \mathrm{CDCl}_{3}\right) \delta 7.11(\mathrm{~d}, \mathrm{~J}=3.4 \mathrm{~Hz}, 1 \mathrm{H}), 6.93(\mathrm{~d}, \mathrm{~J}=3.4 \mathrm{~Hz}, 1 \mathrm{H}), 3.85(\mathrm{~s}$, $3 \mathrm{H}), 1.88(\mathrm{~s}, 2 \mathrm{H}), 1.39(\mathrm{~s}, 12 \mathrm{H}) \mathrm{ppm}$.

${ }^{13} \mathrm{C}$ NMR (101 MHz, $\left.\mathrm{CDCl}_{3}\right) \delta 159.5(\mathrm{C}), 147.4(\mathrm{C}), 122.1(\mathrm{CH}), 118.1(\mathrm{CH}), 71.8(\mathrm{C})$, $51.9\left(\mathrm{O}-\mathrm{CH}_{3}\right), 49.3\left(\mathrm{CH}_{2}\right), 31.7\left(\mathrm{CH}_{3}\right) \mathrm{ppm}$.

HRMS (EI): $\mathrm{m} / \mathrm{z}(\mathrm{M}+\mathrm{H})^{+}=$calcd. for $\mathrm{C}_{13} \mathrm{H}_{20} \mathrm{BO}_{5}$ : 267.1188, found: 267.1183.

3cd. Methyl 5-(5,5-dimethyl-1,3,2-dioxaborinan-2-yl)furan-2-carboxylate<smiles>COC(=O)c1ccc(B2OCC(C)(C)CO2)o1</smiles>

The compound was prepared according to the general procedure using methyl 5-bromofuran-2-carboxylate $(4.1 \mathrm{mg}$, $80 \mu \mathrm{mol}, 1.0$ equiv.), bis(neopentylglycoltao)diboron (45.2 $\mathrm{mg}, 200 \mu \mathrm{mol}, 10.0$ equiv. $)$, dodecanenitrile $(4.4 \mu \mathrm{L}, 20 \mu \mathrm{mol}$, 1.0 equiv.) as internal standard and DIPEA $(4.2 \mu \mathrm{L}, 24 \mu \mathrm{mol}$, 1.2 equiv.) and $\mathrm{G} 1$ ( $10 \mathrm{~g} / \mathrm{L})$. The reaction mixture was irradiated for 20 hours, obtaining $72 \%$ product yield according to GC-FID analysis (60\% isolated yield as white powder).

${ }^{1} \mathrm{H}$ NMR $\left(400 \mathrm{MHz}, \mathrm{CDCl}_{3}\right) \delta 7.17(\mathrm{~d}, \mathrm{~J}=3.5 \mathrm{~Hz}, 1 \mathrm{H}), 6.98(\mathrm{~d}, \mathrm{~J}=3.5 \mathrm{~Hz}, 1 \mathrm{H}), 3.89(\mathrm{~s}$, $3 \mathrm{H}), 3.76(\mathrm{~s}, 4 \mathrm{H}), 1.02(\mathrm{~s}, 6 \mathrm{H}) \mathrm{ppm}$.

${ }^{13} \mathrm{C}$ NMR (101 MHz, $\left.\mathrm{CDCl}_{3}\right) \delta 159.4(\mathrm{C}), 147.9(\mathrm{C}), 122.5(\mathrm{CH}), 118.2(\mathrm{CH}), 73.1(\mathrm{C})$, $52.1\left(\mathrm{O}-\mathrm{CH}_{3}\right), 32.2\left(\mathrm{CH}_{2}\right), 21.7\left(\mathrm{CH}_{3}\right) \mathrm{ppm}$.

HRMS (EI): $\mathrm{m} / \mathrm{z}(\mathrm{M}+\mathrm{H})^{+}=$calcd. for $\mathrm{C}_{11} \mathrm{H}_{15} \mathrm{BO}_{5}: 239.1085$, found: 239.1078 .

\section{PYRROLES}

4aa. 1-(1-Methyl-5-(4,4,5,5-tetramethyl-1,3,2-dioxaborolan-2-yl)-1H-pyrrol-2yl)ethanone<smiles></smiles>

The compound was prepared according to the general procedure using 5-bromo-1-methyl-1H-pyrrole-2-carbaldehyde $(4.0 \mathrm{mg}, 20$ $\mu \mathrm{mol}, 1.0$ equiv.), bis(pinacolato)diboron (50.6 mg, $200 \mu \mathrm{mol}$, 10.0 equiv.), dodecanenitrile $(4.4 \mu \mathrm{L}, 20 \mu \mathrm{mol}, 1.0$ equiv.) as internal standard and DIPEA (4.2 $\mu \mathrm{L}, 24 \mu \mathrm{mol}, 1.2$ equiv.) and G1 (10g/L). The reaction mixture was irradiated for 22 hours, obtaining $91 \%$ product yield according to GC-FID analysis (72\% isolated yield as yellow-pale powder).

${ }^{1} \mathrm{H}$ NMR $\left(400 \mathrm{MHz}, \mathrm{CDCl}_{3}\right) \delta 6.92(\mathrm{~d}, \mathrm{~J}=4.1 \mathrm{~Hz}, 1 \mathrm{H}), 6.70(\mathrm{~d}, \mathrm{~J}=4.0 \mathrm{~Hz}, 1 \mathrm{H}), 4.12(\mathrm{~s}$, $3 \mathrm{H}), 2.46(\mathrm{~s}, 3 \mathrm{H}), 1.33(\mathrm{~s}, 12 \mathrm{H}) \mathrm{ppm}$.

${ }^{13} \mathrm{C}$ NMR (101 MHz, $\left.\mathrm{CDCl}_{3}\right) \delta 189.6(\mathrm{C}), 168.7(\mathrm{C}), 120.3(\mathrm{CH}), 119.0(\mathrm{CH}), 84.0(\mathrm{C})$, $36.7\left(\mathrm{CH}_{3}\right), 28.2\left(\mathrm{CH}_{3}\right), 24.9\left(\mathrm{CH}_{3}\right) \mathrm{ppm}$.

HRMS (El): $\mathrm{m} / \mathrm{z}(\mathrm{M}+\mathrm{H})^{+}=$calcd. for $\mathrm{C}_{13} \mathrm{H}_{20} \mathrm{BNO}_{3}: 250.1609$, found: 250.1604. 
4ab. 1-(1-Methyl-5-(4,4,6-trimethyl-1,3,2-dioxaborinan-2-yl)-1H-pyrrol-2-

yl)ethanone<smiles></smiles>

The compound was prepared according to the general procedure using 5-bromo-1-methyl-1H-pyrrole-2-carbaldehyde $(4.0 \mathrm{mg}, 20$ $\mu \mathrm{mol}, 1.0$ equiv.), bis(pinacolato)diboron ( $50.6 \mathrm{mg}, 200 \mu \mathrm{mol}, 10.0$ equiv.), dodecanenitrile ( $4.4 \mu \mathrm{L}, 20 \mu \mathrm{mol}, 1.0$ equiv.) as internal standard and DIPEA $(4.2 \mu \mathrm{L}, 24 \mu \mathrm{mol}, 1.2$ equiv.) and $\mathrm{G} 1$ (10 $\mathrm{g} / \mathrm{L})$. The reaction mixture was irradiated for 20 hours, obtaining $83 \%$ product yield according to GC-FID analysis (61\% isolated yield as yellow powder).

${ }^{1} \mathrm{H}$ NMR $\left(400 \mathrm{MHz}, \mathrm{CDCl}_{3}\right) \delta 6.90(\mathrm{~d}, \mathrm{~J}=4.0 \mathrm{~Hz}, 1 \mathrm{H}), 6.63(\mathrm{~d}, \mathrm{~J}=4.0 \mathrm{~Hz}, 1 \mathrm{H}), 4.33(\mathrm{~m}$, $1 \mathrm{H}), 4.12(\mathrm{~s}, 3 \mathrm{H}), 2.43(\mathrm{~s}, 3 \mathrm{H}), 1.86(\mathrm{dd}, \mathrm{J}=14.0,2.9 \mathrm{~Hz}, 1 \mathrm{H}), 1.57(\mathrm{t}, \mathrm{J}=13.9 \mathrm{~Hz}, 1 \mathrm{H})$, $1.35(\mathrm{~s}, 6 \mathrm{H}), 1.32(\mathrm{~d}, \mathrm{~J}=6.1 \mathrm{~Hz}, 3 \mathrm{H}) \mathrm{ppm}$.

${ }^{13} \mathrm{C}$ NMR (101 MHz, $\left.\mathrm{CDCl}_{3}\right) \delta 189.4(\mathrm{C}), 165.7(\mathrm{C}), 119.1(\mathrm{CH}), 119.0(\mathrm{CH}), 71.8(\mathrm{C})$, $65.5(\mathrm{CH}), 46.2\left(\mathrm{CH}_{2}\right), 36.4\left(\mathrm{CH}_{3}\right), 31.5\left(\mathrm{CH}_{3}\right), 28.3\left(\mathrm{CH}_{3}\right), 28.2\left(\mathrm{CH}_{3}\right), 23.3\left(\mathrm{CH}_{3}\right) \mathrm{ppm}$. GC-MS (EI): m/z (relative intensity): 249.2 (30) [M+•], 234.1 (50), 134.1 (30), 70.1 (15), $61.0(20), 56.1$ (5), 43.1 (100).

4ac. 1-(1-Methyl-5-(4,4,6,6-tetramethyl-1,3,2-dioxaborinan-2-yl)-1H-pyrrol-2yl)ethanone<smiles></smiles>

The compound was prepared according to the general procedure using 5-bromo-1-methyl-1H-pyrrole-2-carbaldehyde $(4.0 \mathrm{mg}, 20$ umol, 1.0 equiv.), bis(pinacolato)diboron (50.6 mg, $200 \mu \mathrm{mol}, 10.0$ equiv.), dodecanenitrile ( $4.4 \mu \mathrm{L}, 20 \mu \mathrm{mol}, 1.0$ equiv.) as internal standard and DIPEA ( $4.2 \mu \mathrm{L}, 24 \mu \mathrm{mol}, 1.2$ equiv.) and G1 (10g/L). The reaction mixture was irradiated for 20 hours, obtaining $85 \%$ product yield according to GC-FID analysis (63\% isolated yield as yellow-pale powder).

${ }^{1} \mathrm{H}$ NMR $\left(400 \mathrm{MHz}, \mathrm{CDCl}_{3}\right) \delta 6.91(\mathrm{~d}, \mathrm{~J}=4.0 \mathrm{~Hz}, 1 \mathrm{H}), 6.64(\mathrm{~d}, \mathrm{~J}=4.0 \mathrm{~Hz}, 1 \mathrm{H}), 4.14(\mathrm{~s}$, $3 \mathrm{H}), 2.44$ (s, 3H), 1.91 (s, 2H), 1.41 (s, 12H) ppm.

${ }^{13} \mathrm{C}$ NMR (101 MHz, $\left.\mathrm{CDCl}_{3}\right) \delta 189.4(\mathrm{C}), 134.8(\mathrm{C}), 119.1(\mathrm{CH}), 119.0(\mathrm{CH}), 71.5(\mathrm{C})$, $49.0\left(\mathrm{CH}_{2}\right), 36.3\left(\mathrm{CH}_{3}\right), 31.9\left(\mathrm{CH}_{3}\right), 28.2\left(\mathrm{CH}_{3}\right) \mathrm{ppm}$.

GC-MS (El): m/z (relative intensity): 263.1 (5) [M+•], 248.1 (5), 149.0 (5), 88.1 (10), 70.1 (20), 61.1 (30), 43.0 (100).

4ad. 1-(5-(5,5-dimethyl-1,3,2-dioxaborinan-2-yl)-1-methyl-1H-pyrrol-2-yl)ethanone<smiles></smiles>

The compound was prepared according to the general procedure using 5-bromo-1-methyl-1H-pyrrole-2-carbaldehyde (4.0 mg, $20 \mu \mathrm{mol}, 1.0$ equiv.), bis(pinacolato)diboron (50.6 mg, $200 \mu \mathrm{mol}, 10.0$ equiv.), dodecanenitrile $(4.4 \mu \mathrm{L}, 20 \mu \mathrm{mol}, 1.0$ equiv.) as internal standard and DIPEA (4.2 $\mu \mathrm{L}, 24 \mu \mathrm{mol}, 1.2$ equiv.) and $\mathrm{G} 1(10 \mathrm{~g} / \mathrm{L})$. The reaction mixture was irradiated for 20 hours, obtaining $65 \%$ product yield according to GC-FID analysis ( $44 \%$ isolated yield as yellow powder).

${ }^{1} \mathrm{H}$ NMR $\left(400 \mathrm{MHz}, \mathrm{CDCl}_{3}\right) \delta 6.91(\mathrm{~d}, \mathrm{~J}=3.7 \mathrm{~Hz}, 1 \mathrm{H}), 6.64(\mathrm{~d}, \mathrm{~J}=3.8 \mathrm{~Hz}, 1 \mathrm{H}), 4.11$ (s, $3 \mathrm{H}), 3.74(\mathrm{~s}, 4 \mathrm{H}), 2.44(\mathrm{~s}, 3 \mathrm{H}), 1.01(\mathrm{~s}, 6 \mathrm{H}) \mathrm{ppm}$.

${ }^{13} \mathrm{C}$ NMR (101 MHz, $\left.\mathrm{CDCl}_{3}\right) \delta 189.5(\mathrm{C}), 135.0(\mathrm{C}), 119.3(\mathrm{CH}), 119.1(\mathrm{CH}), 73.1(\mathrm{C})$, $36.4\left(\mathrm{CH}_{3}\right), 32.0\left(\mathrm{CH}_{2}\right), 28.2\left(\mathrm{CH}_{3}\right), 21.7\left(\mathrm{CH}_{3}\right) \mathrm{ppm}$.

GC-MS (EI): m/z (relative intensity): 235.1 (15) [M+•], 220.1 (40), 192.0 (5), 148.9 (5), 134.0 (10), 105.9 (5), 88.0 (10), 70.0 (20), 61.0 (20), 52.9 (5), 43.1 (100). 
4 ba. 1-Methyl-5-(4,4,5,5-tetramethyl-1,3,2-dioxaborolan-2-yl)-1H-pyrrole-2carbaldehyde<smiles>C[C@H]1OB(c2ccc(C=O)[nH]2)OC1(C)C</smiles>

The compound was prepared according to the general procedure using 5-bromo-1-methyl-1H-pyrrole-2-carbaldehyde (3.8 mg, $20 \mu \mathrm{mol}, 1.0$ equiv.), bis(pinacolato)diboron (50.6 mg, $200 \mu \mathrm{mol}, 10.0$ equiv.), dodecanenitrile $(4.4 \mu \mathrm{L}, 20 \mu \mathrm{mol}, 1.0$ equiv.) as internal standard and DIPEA ( $4.2 \mu \mathrm{L}, 24 \mu \mathrm{mol}, 1.2$ equiv.) and $\mathrm{G} 1$ (10g/L). The reaction mixture was irradiated for 20 hours, obtaining $72 \%$ product yield according to GC-FID analysis (56\% isolated yield as reddish to black powder).

${ }^{1} \mathrm{H}$ NMR $\left(400 \mathrm{MHz}, \mathrm{CDCl}_{3}\right) \delta 9.56(\mathrm{~s}, 1 \mathrm{H}), 6.81(\mathrm{~d}, \mathrm{~J}=4.0 \mathrm{~Hz}, 1 \mathrm{H}), 6.67(\mathrm{~d}, \mathrm{~J}=4.0 \mathrm{~Hz}$, $1 \mathrm{H}), 4.05(\mathrm{~s}, 3 \mathrm{H}), 1.25(\mathrm{~s}, 12 \mathrm{H}) \mathrm{ppm}$.

${ }^{13} \mathrm{C}$ NMR (101 MHz, $\left.\mathrm{CDCl}_{3}\right) \delta 178.3(\mathrm{C}), 136.0(\mathrm{C}), 123.1(\mathrm{CH}), 112.6(\mathrm{CH}), 84.0(\mathrm{C})$, $36.5\left(\mathrm{CH}_{3}\right), 24.0\left(\mathrm{CH}_{3}\right)$ ppm.

HRMS (EI): $\mathrm{m} / \mathrm{z}(\mathrm{M}+\mathrm{H})^{+}=$calcd. for $\mathrm{C}_{12} \mathrm{H}_{18} \mathrm{BNO}_{3}: 236.0725$, found: 236.0713 .

4bb. carbaldehyde

1-Methyl-5-(4,4,6-trimethyl-1,3,2-dioxaborinan-2-yl)-1H-pyrrole-2-<smiles>CC1CC(C)(C)OB(c2ccc(C=O)n2[14CH3])O1</smiles>

The compound was prepared according to the general procedure using 5-bromo-1-methyl-1H-pyrrole-2-carbaldehyde $(3.8 \mathrm{mg}, 80$ $\mu \mathrm{mol}, 1.0$ equiv.), bis(hexyleneglycolato)diboron (53.0 mg, 200 $\mu \mathrm{mol}, 10.0$ equiv.), dodecanenitrile $(4.4 \mu \mathrm{L}, 20 \mu \mathrm{mol}, 1.0$ equiv.) as internal standard and DIPEA $(4.2 \mu \mathrm{L}, 24 \mu \mathrm{mol}, 1.2$ equiv.) and $\mathrm{G} 1(10 \mathrm{~g} / \mathrm{L})$. The reaction mixture was irradiated for 14 hours, obtaining $81 \%$ product yield according to GC-FID analysis (65\% isolated yield as reddish to black powder).

${ }^{1} \mathrm{H}$ NMR $\left(400 \mathrm{MHz}, \mathrm{CDCl}_{3}\right) \delta 9.61(\mathrm{~s}, 1 \mathrm{H}), 6.86(\mathrm{~d}, \mathrm{~J}=4.0 \mathrm{~Hz}, 1 \mathrm{H}), 6.69(\mathrm{~d}, \mathrm{~J}=4.0 \mathrm{~Hz}$, $1 \mathrm{H}$ ), 4.34 (ddd, J = 11.6, 6.1, $2.9 \mathrm{~Hz}, 1 \mathrm{H}$ ), 4.13 (s, 3H), 1.88 (dd, J = 14.0, $2.9 \mathrm{~Hz}, 1 \mathrm{H}$ ), $1.60(\mathrm{t}, \mathrm{J}=12.7 \mathrm{~Hz}, 1 \mathrm{H}), 1.37(\mathrm{~s}, 6 \mathrm{H}), 1.33(\mathrm{~d}, \mathrm{~J}=6.2 \mathrm{~Hz}, 3 \mathrm{H}) \mathrm{ppm}$.

${ }^{13} \mathrm{C}$ NMR $\left(101 \mathrm{MHz}, \mathrm{CDCl}_{3}\right) \delta 180.3(\mathrm{C}), 135.9(\mathrm{C}), 123.5(\mathrm{CH}), 120.0(\mathrm{CH}), 109.7(\mathrm{CH})$, $71.9(\mathrm{C}), 65.6(\mathrm{CH}), 46.1\left(\mathrm{CH}_{2}\right), 35.5\left(\mathrm{CH}_{3}\right), 31.3\left(\mathrm{CH}_{3}\right), 28.3\left(\mathrm{CH}_{3}\right), 23.2\left(\mathrm{CH}_{3}\right) \mathrm{ppm}$.

HRMS (EI): $\mathrm{m} / \mathrm{z}(\mathrm{M}+\mathrm{H})^{+}=$calcd. for $\mathrm{C}_{12} \mathrm{H}_{18} \mathrm{BNO}_{3}: 236.1453$, found: 236.1445 .

4bc.

1-Methyl-5-(4,4,6,6-tetramethyl-1,3,2-dioxaborinan-2-yl)-1H-pyrrole-2carbaldehyde<smiles>Cn1c(C=O)ccc1B1OC(C)(C)CC(C)(C)O1</smiles>

The compound was prepared according to the general procedure using 5-bromo-1-methyl-1H-pyrrole-2-carbaldehyde $(3.8 \mathrm{mg}, 80$ umol, 1.0 equiv. $), \quad 4,4,4^{\prime}, 4^{\prime}, 6,6,6^{\prime}, 6^{\prime}$-octamethyl-2,2'-bi(1,3,2dioxaborinane) (56.4 mg, $200 \mu \mathrm{mol}, 10.0$ equiv.), dodecanenitrile $(4.4 \mu \mathrm{L}, 20 \mu \mathrm{mol}, 1.0$ equiv.) as internal standard and DIPEA (4.2 $\mu \mathrm{L}, 24 \mu \mathrm{mol}, 1.2$ equiv.) and $\mathrm{G} 1(10 \mathrm{~g} / \mathrm{L})$. The reaction mixture was irradiated for 20 hours, obtaining $74 \%$ product yield according to GC-FID analysis ( $59 \%$ isolated yield as reddish to black powder).

${ }^{1} \mathrm{H}$ NMR $\left(400 \mathrm{MHz}, \mathrm{CDCl}_{3}\right) \delta 9.59(\mathrm{~s}, 1 \mathrm{H}), 6.85(\mathrm{~d}, \mathrm{~J}=4.0 \mathrm{~Hz}, 1 \mathrm{H}), 6.68(\mathrm{~d}, \mathrm{~J}=4.0 \mathrm{~Hz}$, $1 \mathrm{H}), 4.13(\mathrm{~s}, 3 \mathrm{H}), 1.91(\mathrm{~s}, 2 \mathrm{H}), 1.40(\mathrm{~s}, 12 \mathrm{H}) \mathrm{ppm}$.

${ }^{13} \mathrm{C} \mathrm{NMR}\left(101 \mathrm{MHz}, \mathrm{CDCl}_{3}\right) \delta 179.7(\mathrm{C}), 135.8(\mathrm{C}), 123.4(\mathrm{CH}), 109.6(\mathrm{CH}), 71.6(\mathrm{C})$, $49.0\left(\mathrm{CH}_{2}\right), 35.5\left(\mathrm{CH}_{3}\right), 31.8\left(\mathrm{CH}_{3}\right) \mathrm{ppm}$.

HRMS (EI): $\mathrm{m} / \mathrm{z}(\mathrm{M}+\mathrm{H})^{+}=$calcd. for $\mathrm{C}_{13} \mathrm{H}_{20} \mathrm{BNO}_{3}: 250.1609$, found: 250.1604 . 
4bd. 5-(5,5-dimethyl-1,3,2-dioxaborinan-2-yl)-1-methyl-1H-pyrrole-2-carbaldehyde<smiles></smiles>

The compound was prepared according to the general procedure using 5-bromo-1-methyl-1H-pyrrole-2-carbaldehyde (3.8 mg, $80 \mu \mathrm{mol}, 1.0$ equiv.), bis(neopentylglycoltao)diboron (45.2 mg, $200 \mu \mathrm{mol}, 10.0$ equiv.), dodecanenitrile $(4.4 \mu \mathrm{L}, 20$ $\mu \mathrm{mol}, 1.0$ equiv.) as internal standard and DIPEA $(4.2 \mu \mathrm{L}, 24$ $\mu \mathrm{mol}, 1.2$ equiv.) and $\mathrm{G} 1$ ( $10 \mathrm{~g} / \mathrm{L})$. The reaction mixture was irradiated for 14 hours, obtaining $64 \%$ product yield according to GC-FID analysis ( $51 \%$ isolated yield reddish to black powder).

${ }^{1} \mathrm{H}$ NMR $\left(400 \mathrm{MHz}, \mathrm{CDCl}_{3}\right) 9.59(\mathrm{~s}, 1 \mathrm{H}), 6.85(\mathrm{~d}, \mathrm{~J}=4.0 \mathrm{~Hz}, 1 \mathrm{H}), 6.68(\mathrm{~d}, \mathrm{~J}=4.0 \mathrm{~Hz}$, $1 \mathrm{H}), 4.11(\mathrm{~s}, 3 \mathrm{H}), 3.73(\mathrm{~s}, 4 \mathrm{H}), 1.00(\mathrm{~s}, 6 \mathrm{H}) \mathrm{ppm}$.

${ }^{13} \mathrm{C}$ NMR (101 MHz, $\left.\mathrm{CDCl}_{3}\right) \delta 179.7(\mathrm{C}), 132.2(\mathrm{C}), 124.3(\mathrm{CH}), 109.6(\mathrm{CH}), 73.0(\mathrm{C})$, $36.4\left(\mathrm{CH}_{3}\right), 32.1\left(\mathrm{CH}_{2}\right), 21.7\left(\mathrm{CH}_{3}\right) \mathrm{ppm}$.

HRMS (EI): $\mathrm{m} / \mathrm{z}(\mathrm{M}+\mathrm{H})^{+}=$calcd. for $\mathrm{C}_{11} \mathrm{H}_{16} \mathrm{BNO}_{3}: 223.0944$, found: 223.0956 .

4ca. Methyl 1-methyl-5-(4,4,5,5-tetramethyl-1,3,2-dioxaborolan-2-yl)-1H-pyrrole-2carboxylate<smiles></smiles>

The compound (CAS 2377607-43-5) was prepared according to the general procedure using methyl 5-bromo-1-methyl-1Hpyrrole-2-carboxylate $(4.4 \mathrm{mg}, 20 \mu \mathrm{mol}, 1.0$ equiv.), bis(pinacolato)diboron (50.6 mg, $200 \mu \mathrm{mol}, 10.0$ equiv.), dodecanenitrile ( $4.4 \mu \mathrm{L}, 20 \mu \mathrm{mol}, 1.0$ equiv.) as internal standard and DIPEA (4.2 $\mu \mathrm{L}, 24$ umol, 1.2 equiv.) and $\mathrm{G} 1(10 \mathrm{~g} / \mathrm{L})$. The reaction mixture was irradiated for 18 hours, obtaining $69 \%$ product yield according to GC-FID analysis (55\% isolated yield as yellowpale oil).

${ }^{1} \mathrm{H}$ NMR $\left(400 \mathrm{MHz}, \mathrm{CDCl}_{3}\right) \delta 6.91(\mathrm{~d}, \mathrm{~J}=4.0 \mathrm{~Hz}, 1 \mathrm{H}), 6.70(\mathrm{~d}, \mathrm{~J}=4.0 \mathrm{~Hz}, 1 \mathrm{H}), 4.13(\mathrm{~s}$, $3 \mathrm{H}), 3.81(\mathrm{~s}, 3 \mathrm{H}), 1.32(\mathrm{~s}, 12 \mathrm{H}) \mathrm{ppm}$.

${ }^{13} \mathrm{C}$ NMR $\left(101 \mathrm{MHz}, \mathrm{CDCl}_{3}\right) \delta 161.91(\mathrm{C}), 127.49(\mathrm{C}), 120.41(\mathrm{CH}), 117.29(\mathrm{CH}), 83.85$ (C), $51.27\left(\mathrm{O}-\mathrm{CH}_{3}\right), 35.92\left(\mathrm{CH}_{3}\right), 24.92\left(\mathrm{CH}_{3}\right) \mathrm{ppm}$.

HRMS (El): $\mathrm{m} / \mathrm{z}(\mathrm{M}+\mathrm{H})^{+}=$calcd. for $\mathrm{C}_{13} \mathrm{H}_{21} \mathrm{BNO}_{4}: 266.1559$, found: 266.1564.

4cb. Methyl 1-methyl-5-(4,4,6-trimethyl-1,3,2-dioxaborinan-2-yl)-1H-pyrrole-2carboxylate<smiles>COC(=O)c1ccc(B2OC(C)CC(C)(C)O2)n1C</smiles>

The compound was prepared according to the general procedure using methyl 5-bromo-1-methyl-1H-pyrrole-2carboxylate $(4.4 \mathrm{mg}, \quad 80 \mu \mathrm{mol}, \quad 1.0$ equiv.), bis(hexyleneglycolato)diboron $(53.0 \mathrm{mg}, 200 \mu \mathrm{mol}, 10.0$ equiv.), dodecanenitrile (4.4 $\mu \mathrm{L}, 20 \mu \mathrm{mol}, 1.0$ equiv.) as internal standard and DIPEA (4.2 $\mu \mathrm{L}, 24 \mu \mathrm{mol}, 1.2$ equiv.) and $\mathrm{G} 1$ (10g/L). The reaction mixture was irradiated for 19 hours, obtaining $76 \%$ product yield according to GC-FID analysis (62\% isolated yield as yellow-pale oil).

${ }^{1} \mathrm{H}$ NMR $\left(400 \mathrm{MHz}, \mathrm{CDCl}_{3}\right) \delta 6.89$ (d, J = 3.9 Hz, 1H), 6.64 (d, J = 3.9 Hz, 1H), 4.32 (ddd, $\mathrm{J}=11.7,6.0,3.0 \mathrm{~Hz}, 1 \mathrm{H}), 4.12(\mathrm{~s}, 3 \mathrm{H}), 3.80(\mathrm{~s}, 3 \mathrm{H}), 1.85(\mathrm{dd}, \mathrm{J}=13.9,2.9 \mathrm{~Hz}, 1 \mathrm{H}), 1.63$ $-1.52(\mathrm{~m}, 1 \mathrm{H}), 1.36(\mathrm{~s}, 6 \mathrm{H}), 1.32(\mathrm{~d}, \mathrm{~J}=6.2 \mathrm{~Hz}, 3 \mathrm{H}) \mathrm{ppm}$.

${ }^{13} \mathrm{C}$ NMR (101 MHz, $\left.\mathrm{CDCl}_{3}\right) \delta 162.1(\mathrm{C}), 126.7(\mathrm{C}), 119.1(\mathrm{CH}), 117.1(\mathrm{CH}), 71.6(\mathrm{C})$, $65.4(\mathrm{CH}), 51.1\left(\mathrm{O}-\mathrm{CH}_{3}\right), 46.1\left(\mathrm{CH}_{2}\right), 35.5\left(\mathrm{CH}_{3}\right), 31.4\left(\mathrm{CH}_{3}\right), 28.3\left(\mathrm{CH}_{3}\right), 23.3\left(\mathrm{CH}_{3}\right) \mathrm{ppm}$. GC-MS (El): m/z (relative intensity): 265.1 (60) [M+•], 233.9 (30), 206.8 (20), 166.0 (50), 152.0 (20), 134.1 (40), 108.0 (30), 72.1 (20), 43.1 (100). 
4cc. Methyl 1-methyl-5-(4,4,6,6-tetramethyl-1,3,2-dioxaborinan-2-yl)-1H-pyrrole-2carboxylate<smiles></smiles>

The compound was prepared according to the general procedure using methyl 5-bromo-1-methyl-1H-pyrrole-2carboxylate (4.4 mg, 80 Hmol, 1.0 equiv.), 4,4,4',4',6,6,6',6'octamethyl-2,2'-bi(1,3,2-dioxaborinane) (56.4 mg, $200 \mu \mathrm{mol}$, 10.0 equiv.), dodecanenitrile $(4.4 \mu \mathrm{L}, 20 \mu \mathrm{mol}, 1.0$ equiv.) as internal standard and DIPEA (4.2 $\mu \mathrm{L}, 24 \mu \mathrm{mol}, 1.2$ equiv.) and $\mathrm{G} 1$ (10g/L). The reaction mixture was irradiated for 15 hours, obtaining $77 \%$ product yield according to GC-FID analysis (63\% isolated yield as yellow-pale oil).

${ }^{1} \mathrm{H}$ NMR $\left(400 \mathrm{MHz}, \mathrm{CDCl}_{3}\right) \delta 6.89(\mathrm{~d}, \mathrm{~J}=3.9 \mathrm{~Hz}, 1 \mathrm{H}), 6.65(\mathrm{~d}, \mathrm{~J}=3.9 \mathrm{~Hz}, 1 \mathrm{H}), 4.14(\mathrm{~s}$, $3 \mathrm{H}), 3.80(\mathrm{~s}, 3 \mathrm{H}), 1.91(\mathrm{~s}, 2 \mathrm{H}), 1.41(\mathrm{~s}, 12 \mathrm{H}) \mathrm{ppm}$.

${ }^{13} \mathrm{C}$ NMR $\left(101 \mathrm{MHz}, \mathrm{CDCl}_{3}\right) \delta 162.2(\mathrm{C}), 120.0(\mathrm{C}), 119.1(\mathrm{CH}), 117.1(\mathrm{CH}), 71.4(\mathrm{C})$, $51.1\left(\mathrm{O}-\mathrm{CH}_{3}\right), 49.1\left(\mathrm{CH}_{2}\right), 35.6\left(\mathrm{CH}_{3}\right), 31.9\left(\mathrm{CH}_{3}\right) \mathrm{ppm}$.

HRMS (El): $\mathrm{m} / \mathrm{z}(\mathrm{M}+\mathrm{H})^{+}=$calcd. for $\mathrm{C}_{14} \mathrm{H}_{22} \mathrm{BNO}_{4}: 280.1715$, found: 280.1710 .

4cd. Methyl 5-(5,5-dimethyl-1,3,2-dioxaborinan-2-yl)-1-methyl-1H-pyrrole-2carboxylate<smiles></smiles>

The compound was prepared according to the general procedure using methyl 5-bromo-1-methyl-1H-pyrrole-2carboxylate $(4.4 \mathrm{mg}, \quad 80 \mu \mathrm{mol}, 1.0 \quad$ equiv. $)$, bis(neopentylglycoltao)diboron $(45.2 \mathrm{mg}, 200 \mu \mathrm{mol}, 10.0$ equiv.), dodecanenitrile $(4.4 \mu \mathrm{L}, 20 \mu \mathrm{mol}, 1.0$ equiv.) as internal standard and DIPEA (4.2 $\mu \mathrm{L}, 24 \mu \mathrm{mol}, 1.2$ equiv.) and $\mathrm{G} 1$ (10g/L). The reaction mixture was irradiated for 19 hours, obtaining $44 \%$ product yield according to GC-FID analysis ( $36 \%$ isolated yield as yellow-pale oil).

${ }^{1} \mathrm{H}$ NMR $\left(400 \mathrm{MHz}, \mathrm{CDCl}_{3}\right) \delta 6.90(\mathrm{~d}, \mathrm{~J}=3.9 \mathrm{~Hz}, 1 \mathrm{H}), 6.65(\mathrm{~d}, \mathrm{~J}=3.9 \mathrm{~Hz}, 1 \mathrm{H}), 4.12(\mathrm{~s}$, $3 \mathrm{H}), 3.80(\mathrm{~s}, 3 \mathrm{H}), 3.74(\mathrm{~s}, 4 \mathrm{H}), 1.02(\mathrm{~s}, 6 \mathrm{H}) \mathrm{ppm}$.

${ }^{13} \mathrm{C}$ NMR (101 MHz, CDCl $)_{3} \delta 162.1(\mathrm{C}), 119.4(\mathrm{C}), 117.2(\mathrm{CH}), 111.8(\mathrm{CH}), 73.1\left(\mathrm{CH}_{2}\right)$, $51.2\left(\mathrm{O}-\mathrm{CH}_{3}\right), 35.8\left(\mathrm{CH}_{3}\right), 32.1\left(\mathrm{CH}_{3}\right), 21.6\left(\mathrm{CH}_{3}\right) \mathrm{ppm}$.

HRMS (EI): $\mathrm{m} / \mathrm{z}(\mathrm{M}+\mathrm{H})^{+}=$calcd. for $\mathrm{C}_{12} \mathrm{H}_{19} \mathrm{BNO}_{4}: 252.1402$, found: 252.1406.

\section{SELENOPHENES}

5aa. 1-(5-(4,4,5,5-tetramethyl-1,3,2-dioxaborolan-2-yl)selenophen-2-yl)ethenone<smiles>CC(=O)c1ccc(B2OC(C)(C)C(C)(C)O2)s1</smiles>

The compound was prepared according to the general procedure using 1-(5-bromoselenophen-2-yl)ethanone $(5.0 \mathrm{mg}, 20 \mu \mathrm{mol}$, 1.0 equiv.), bis(pinacolato)diboron $(50.6 \mathrm{mg}, 200 \mu \mathrm{mol}, 10.0$ equiv.), dodecanenitrile ( $4.4 \mu \mathrm{L}, 20 \mu \mathrm{mol}, 1.0$ equiv.) as internal standard and DIPEA (4.2 $\mu \mathrm{L}, 24 \mu \mathrm{mol}, 1.2$ equiv.) and $\mathrm{G} 1(10 \mathrm{~g} / \mathrm{L})$. The reaction mixture was irradiated for 16 hours, obtaining $84 \%$ product yield according to GC-FID analysis ( $70 \%$ isolated yield as yellow-brown powder).

${ }^{1} \mathrm{H}$ NMR $\left(400 \mathrm{MHz}, \mathrm{CDCl}_{3}\right) \delta 7.94(\mathrm{~d}, \mathrm{~J}=3.9 \mathrm{~Hz}, 1 \mathrm{H}), 7.90(\mathrm{~d}, \mathrm{~J}=3.9 \mathrm{~Hz}, 1 \mathrm{H}), 2.58$ (s, $3 \mathrm{H}), 1.34(\mathrm{~s}, 12 \mathrm{H}) \mathrm{ppm}$.

${ }^{13} \mathrm{C}$ NMR (101 MHz, CDCl $\left.{ }_{3}\right) \delta 192.2(\mathrm{C}), 156.8(\mathrm{C}), 140.0(\mathrm{CH}), 135.6(\mathrm{CH}), 84.8(\mathrm{C})$, $26.9\left(\mathrm{CH}_{3}\right), 24.9\left(\mathrm{CH}_{3}\right)$ ppm.

HRMS (El): $\mathrm{m} / \mathrm{z}(\mathrm{M}+\mathrm{H})^{+}=$calcd. for $\mathrm{C}_{12} \mathrm{H}_{17} \mathrm{BO}_{3} \mathrm{Se}: 301.0509$, found: 301.0515 . 
5ab. 1-(5-(4,4,6-trimethyl-1,3,2-dioxaborinan-2-yl)selenophen-2-yl)ethanone<smiles>CC(=O)c1ccc(B2OC(C)CC(C)(C)O2)[se]1</smiles>

The compound was prepared according to the general procedure using 1-(5-bromoselenophen-2-yl)ethanone (5.0 mg, $20 \mu \mathrm{mol}, 1.0$ equiv.), bis(pinacolato)diboron (50.6 mg, $200 \mu \mathrm{mol}, 10.0$ equiv.), dodecanenitrile $(4.4 \mu \mathrm{L}, 20 \mu \mathrm{mol}, 1.0$ equiv.) as internal standard and DIPEA (4.2 $\mu \mathrm{L}, 24 \mu \mathrm{mol}, 1.2$ equiv.) and $\mathrm{G} 1$ (10g/L). The reaction mixture was irradiated for 16 hours, obtaining $86 \%$ product yield according to GC-FID analysis (73\% isolated yield as yellow oil).

${ }^{1} \mathrm{H}$ NMR $\left(400 \mathrm{MHz}, \mathrm{CDCl}_{3}\right) \delta 7.91(\mathrm{~d}, \mathrm{~J}=3.8 \mathrm{~Hz}, 1 \mathrm{H}), 7.81$ (d, J = $\left.3.9 \mathrm{~Hz}, 1 \mathrm{H}\right), 4.34$ (dtt, $\mathrm{J}=12.2,6.1,3.1 \mathrm{~Hz}, 1 \mathrm{H}), 2.56(\mathrm{~s}, 3 \mathrm{H}), 1.87(\mathrm{dd}, \mathrm{J}=14.0,2.9 \mathrm{~Hz}, 1 \mathrm{H}), 1.67-1.56(\mathrm{~m}$, $1 \mathrm{H}), 1.36(\mathrm{~s}, 6 \mathrm{H}), 1.33(\mathrm{~d}, \mathrm{~J}=6.2 \mathrm{~Hz}, 3 \mathrm{H}) \mathrm{ppm}$.

${ }^{13} \mathrm{C}$ NMR (101 MHz, CDCl ${ }_{3}$ ) ठ $192.3(\mathrm{C}), 155.1(\mathrm{C}), 137.9(\mathrm{CH}), 135.7(\mathrm{CH}), 72.1(\mathrm{C})$, $65.8(\mathrm{CH}), 46.1\left(\mathrm{CH}_{2}\right), 31.2\left(\mathrm{CH}_{3}\right), 28.2\left(\mathrm{CH}_{3}\right), 26.8\left(\mathrm{CH}_{3}\right), 23.2\left(\mathrm{CH}_{3}\right) \mathrm{ppm}$.

HRMS (El): $\mathrm{m} / \mathrm{z}(\mathrm{M}+\mathrm{H})^{+}=$calcd. for $\mathrm{C}_{12} \mathrm{H}_{17} \mathrm{BO}_{3} \mathrm{Se}: 301.0509$, found: 301.0502 .

\section{5ac. 1-(5-(4,4,6,6-tetramethyl-1,3,2-dioxaborinan-2-yl)selenophen-2-yl)ethanone}<smiles>CC(=O)c1ccc(B2OC(C)(C)CC(C)(C)O2)s1</smiles>

The compound was prepared according to the general procedure using 1-(5-bromoselenophen-2-yl)ethanone $(5.0 \mathrm{mg}, 20 \mu \mathrm{mol}, 1.0$ equiv.), bis(pinacolato)diboron (50.6 mg, $200 \mu \mathrm{mol}, 10.0$ equiv.), dodecanenitrile $(4.4 \mu \mathrm{L}, 20 \mu \mathrm{mol}, 1.0$ equiv.) as internal standard and DIPEA (4.2 $\mu \mathrm{L}, 24 \mu \mathrm{mol}, 1.2$ equiv.) and $\mathrm{G} 1$ (10g/L). The reaction mixture was irradiated for 16 hours, obtaining $88 \%$ product yield according to GC-FID analysis (69\% isolated yield as yellow oil).

${ }^{1} \mathrm{H}$ NMR $\left(400 \mathrm{MHz}, \mathrm{CDCl}_{3}\right) \delta 7.92(\mathrm{~d}, \mathrm{~J}=3.9 \mathrm{~Hz}, 1 \mathrm{H}), 7.83(\mathrm{~d}, \mathrm{~J}=3.9 \mathrm{~Hz}, 1 \mathrm{H}), 2.57$ (s, $3 \mathrm{H}), 1.92(\mathrm{~s}, 2 \mathrm{H}), 1.42(\mathrm{~s}, 12 \mathrm{H}) \mathrm{ppm}$.

${ }^{13} \mathrm{C}$ NMR (101 MHz, CDCl $\left.{ }_{3}\right) \delta 192.3(\mathrm{C}), 155.0(\mathrm{C}), 137.8(\mathrm{CH}), 135.7(\mathrm{CH}), 71.9(\mathrm{C})$, $49.1\left(\mathrm{CH}_{2}\right), 31.8\left(\mathrm{CH}_{3}\right), 26.8\left(\mathrm{CH}_{3}\right) \mathrm{ppm}$.

HRMS (EI): $\mathrm{m} / \mathrm{z}(\mathrm{M}+\mathrm{H})^{+}=$calcd. for $\mathrm{C}_{13} \mathrm{H}_{19} \mathrm{BO}_{3} \mathrm{Se}: 315.0665$, found: 315.0658 .

\section{5ad. 1-(5-(5,5-dimethyl-1,3,2-dioxaborinan-2-yl)selenophen-2-yl)ethanone}<smiles>CC(=O)c1ccc(B2OCC(C)(C)CO2)[se]1</smiles>

The compound was prepared according to the general procedure using 1-(5-bromoselenophen-2-yl)ethanone (5.0 mg, $20 \mu \mathrm{mol}, 1.0$ equiv.), bis(pinacolato)diboron (50.6 mg, $200 \mu \mathrm{mol}$, 10.0 equiv.), dodecanenitrile ( $4.4 \mu \mathrm{L}, 20 \mu \mathrm{mol}, 1.0$ equiv.) as internal standard and DIPEA (4.2 $\mu \mathrm{L}, 24 \mu \mathrm{mol}, 1.2$ equiv.) and $\mathrm{G} 1(10 \mathrm{~g} / \mathrm{L})$. The reaction mixture was irradiated for 22 hours, obtaining $68 \%$ product yield according to GC-FID analysis (51\% isolated yield as yellow oil).

${ }^{1} \mathrm{H}$ NMR $\left(400 \mathrm{MHz}, \mathrm{CDCl}_{3}\right) \delta 7.93(\mathrm{~d}, \mathrm{~J}=3.9 \mathrm{~Hz}, 1 \mathrm{H}), 7.84(\mathrm{~d}, \mathrm{~J}=3.9 \mathrm{~Hz}, 1 \mathrm{H}), 3.76(\mathrm{~s}$, $4 \mathrm{H}), 2.58(\mathrm{~s}, 3 \mathrm{H}), 1.03(\mathrm{~s}, 6 \mathrm{H}) \mathrm{ppm}$.

${ }^{13} \mathrm{C}$ NMR $\left(101 \mathrm{MHz}, \mathrm{CDCl}_{3}\right) \delta 207.1(\mathrm{C}), 155.1(\mathrm{C}), 138.4(\mathrm{CH}), 135.8(\mathrm{CH}), 72.7(\mathrm{C})$, $31.1\left(\mathrm{CH}_{2}\right), 26.8\left(\mathrm{CH}_{3}\right), 22.0\left(\mathrm{CH}_{3}\right) \mathrm{ppm}$.

HRMS (EI): $\mathrm{m} / \mathrm{z}(\mathrm{M}+\mathrm{H})^{+}=$calcd. for $\mathrm{C}_{11} \mathrm{H}_{15} \mathrm{BO}_{3} \mathrm{Se}: 285.0060$, found: 285.2277 . 


\section{Diboron esters}

\section{NMR Spectra}

${ }^{1} \mathrm{H} 400 \mathrm{MHz}, \mathrm{ACN}-\mathrm{d} 3$

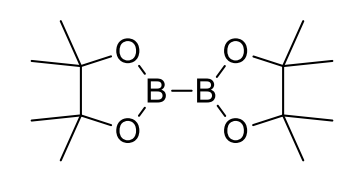
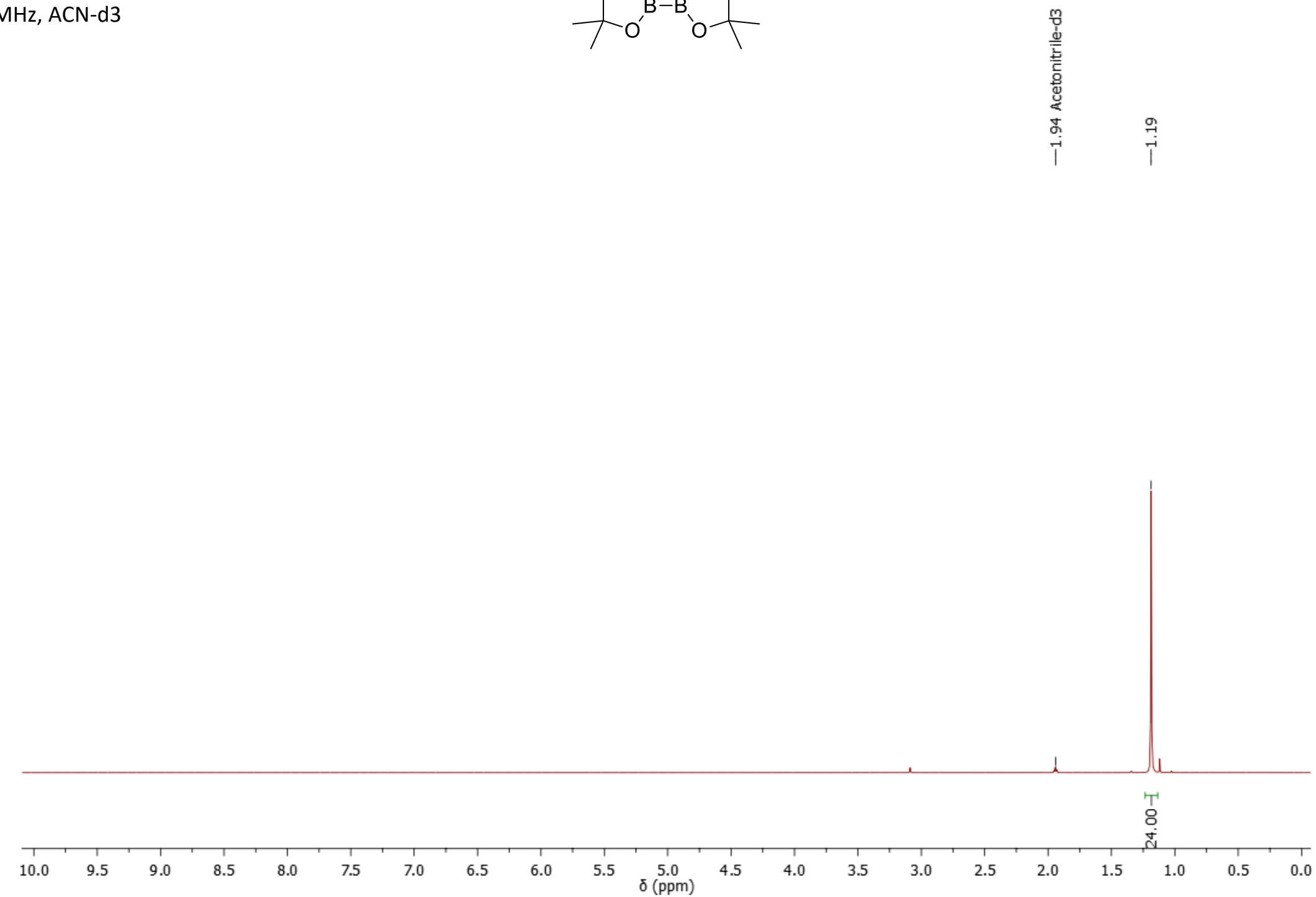


\section{Diboron esters}

${ }^{13} \mathrm{C} 101 \mathrm{MHz}$

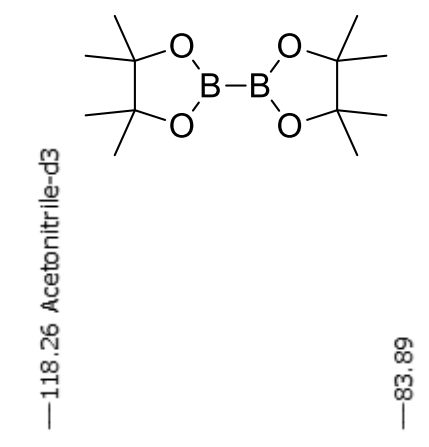

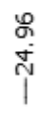

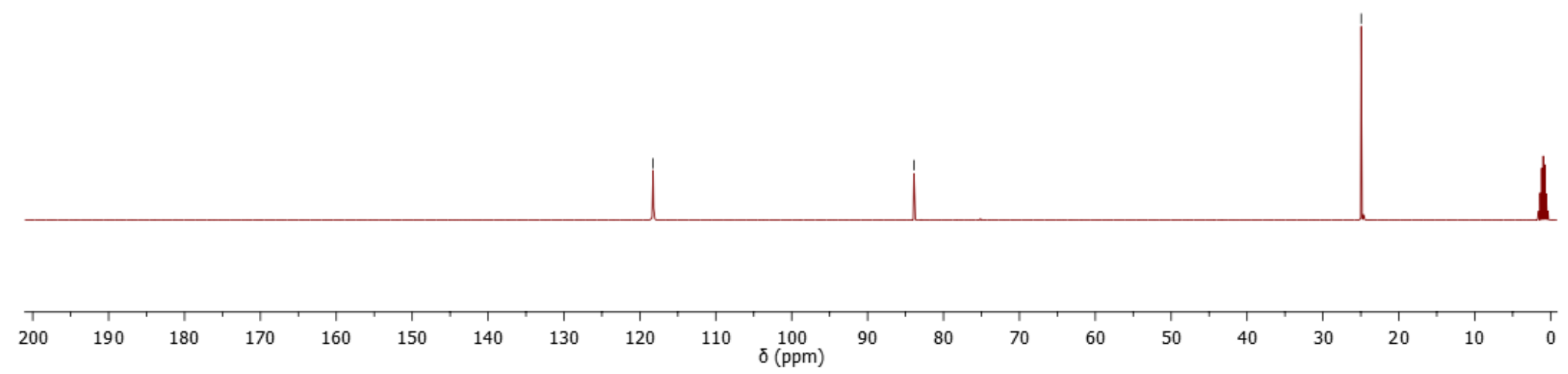


Diboron esters

${ }^{1} \mathrm{H} 400 \mathrm{MHz}, \mathrm{CDCl}_{3}$

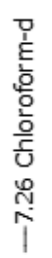

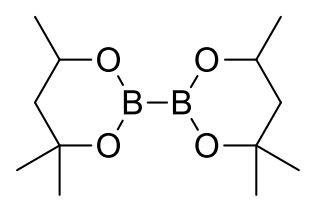

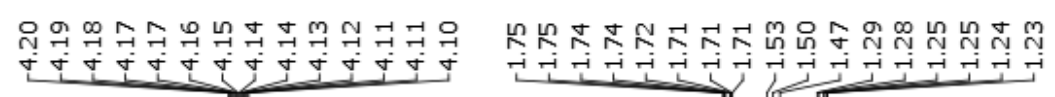

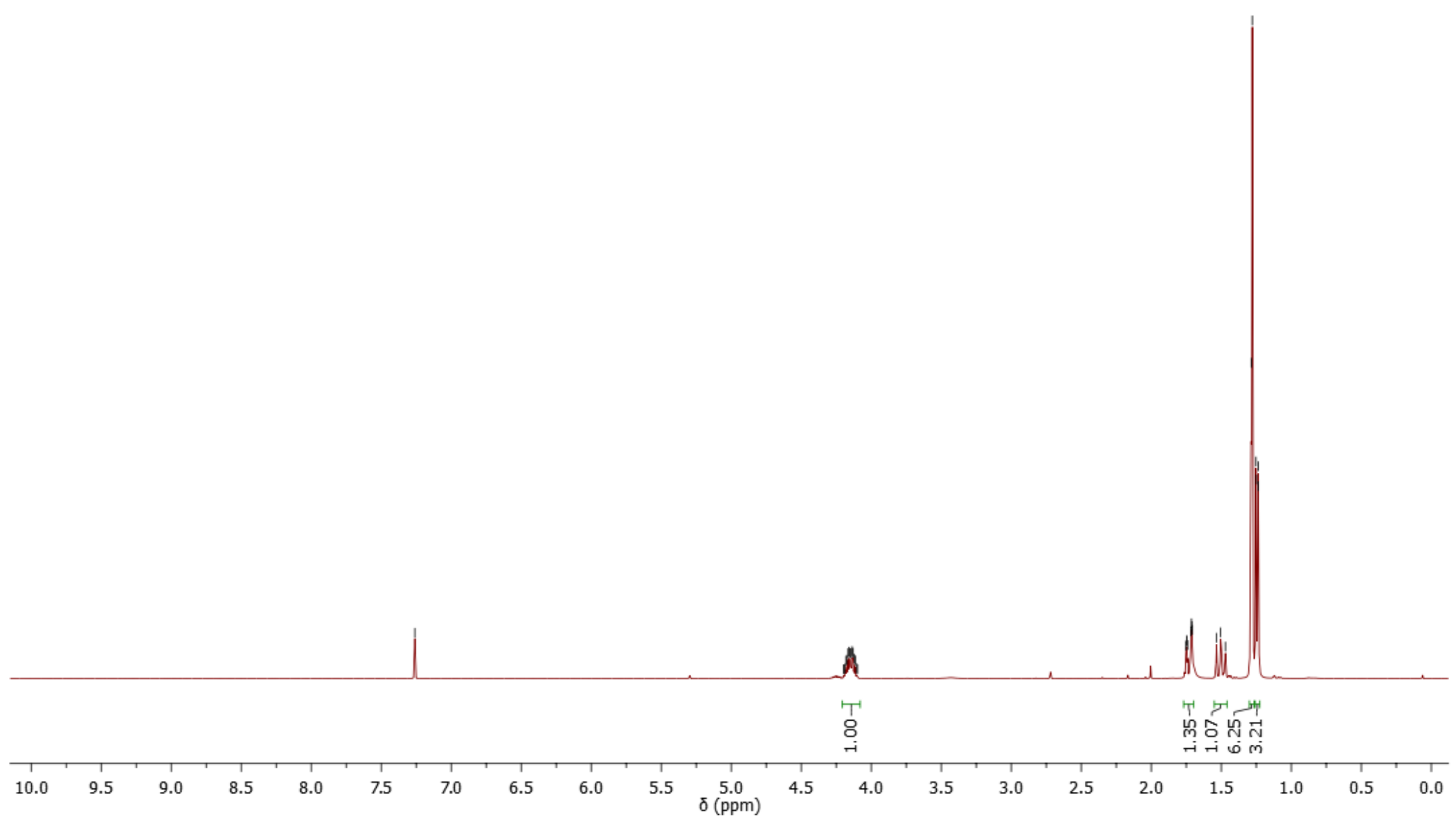


Diboron esters

${ }^{13} \mathrm{C} 101 \mathrm{MHz}$

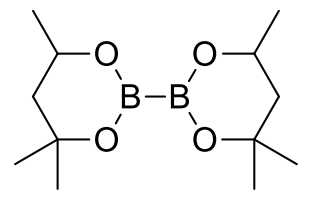

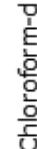

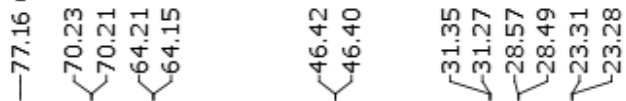

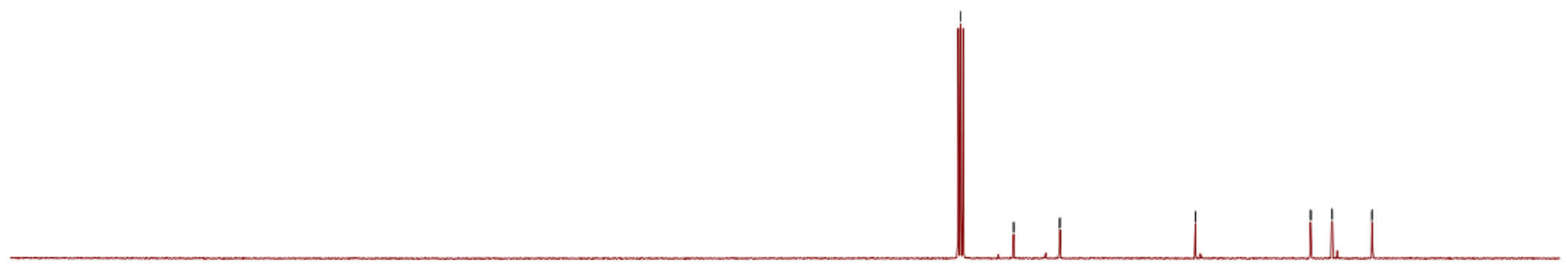




\section{Diboron esters}

${ }^{1} \mathrm{H} 400 \mathrm{MHz}, \mathrm{CDCl}_{3}$

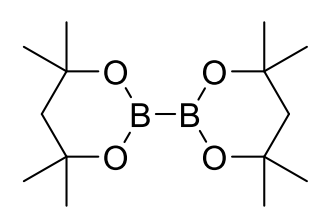

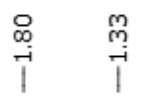

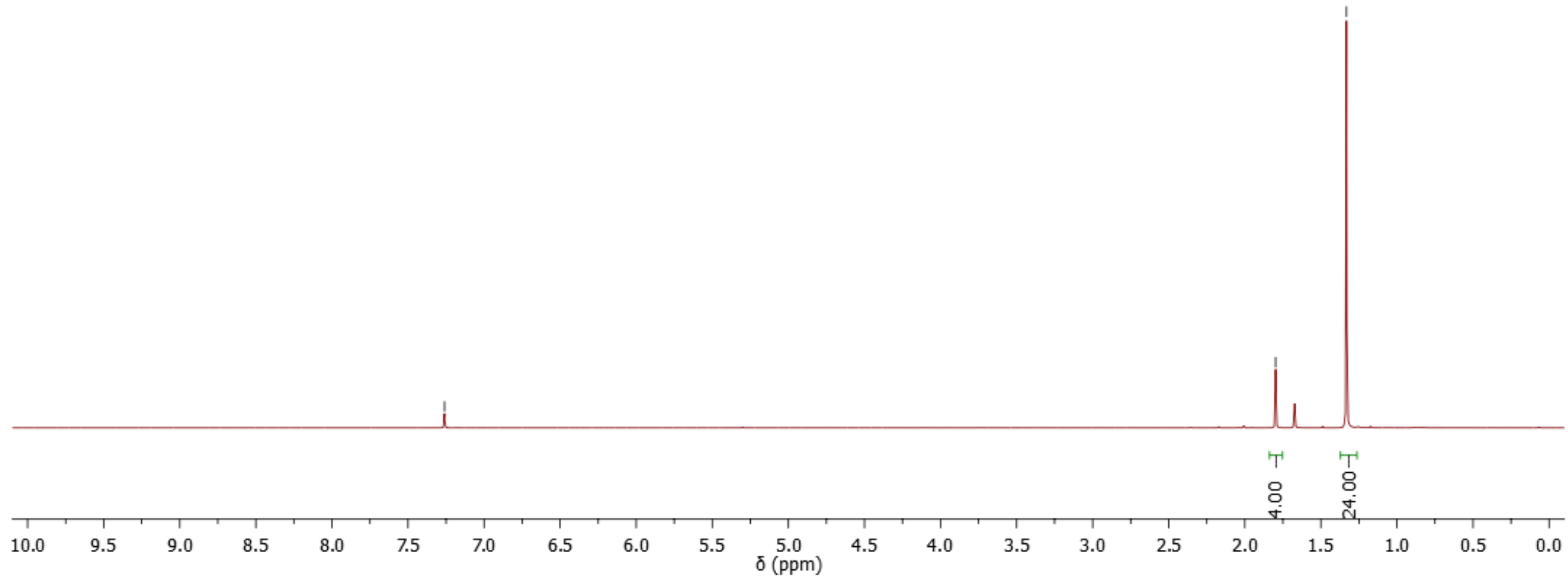




\section{Diboron esters}

${ }^{13} \mathrm{C} 101 \mathrm{MHz}$
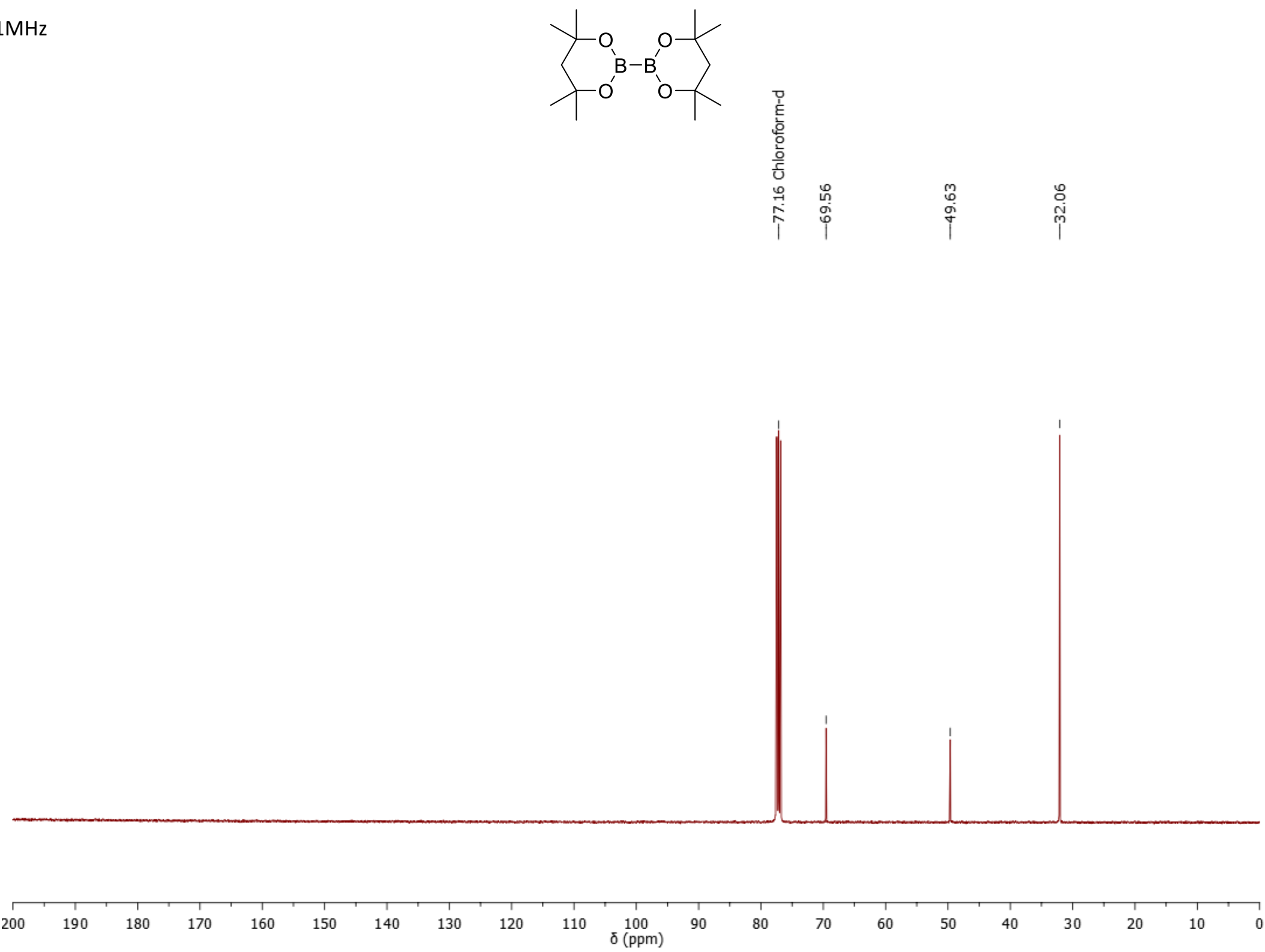


\section{Diboron esters}

${ }^{1} \mathrm{H} 400 \mathrm{MHz}, \mathrm{CDCl}_{3}$
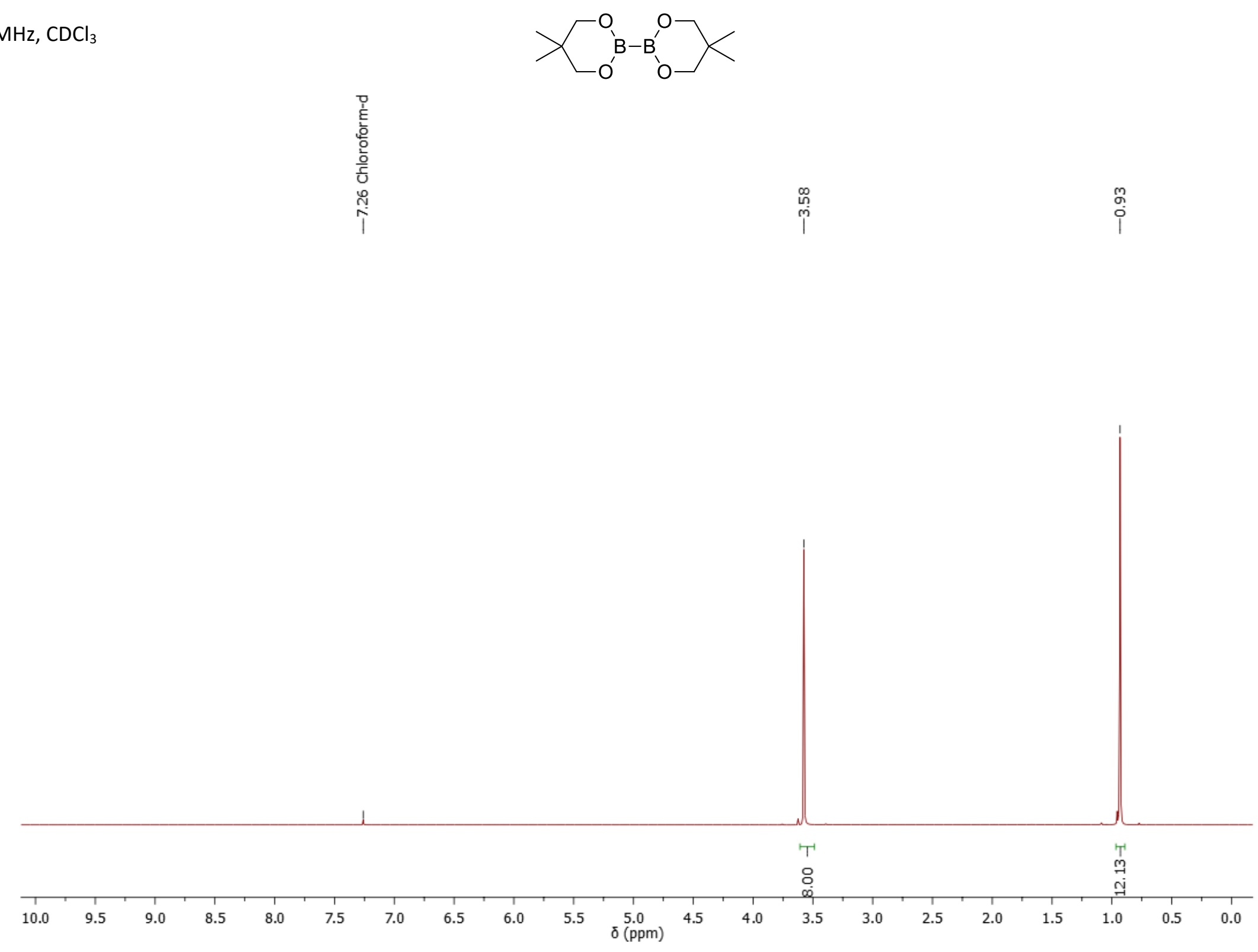


\section{Diboron esters}

${ }^{13} \mathrm{C} 101 \mathrm{MHz}$

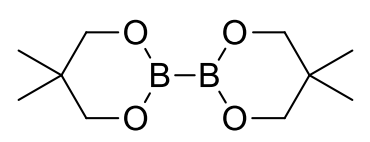

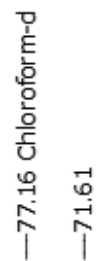

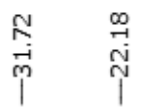
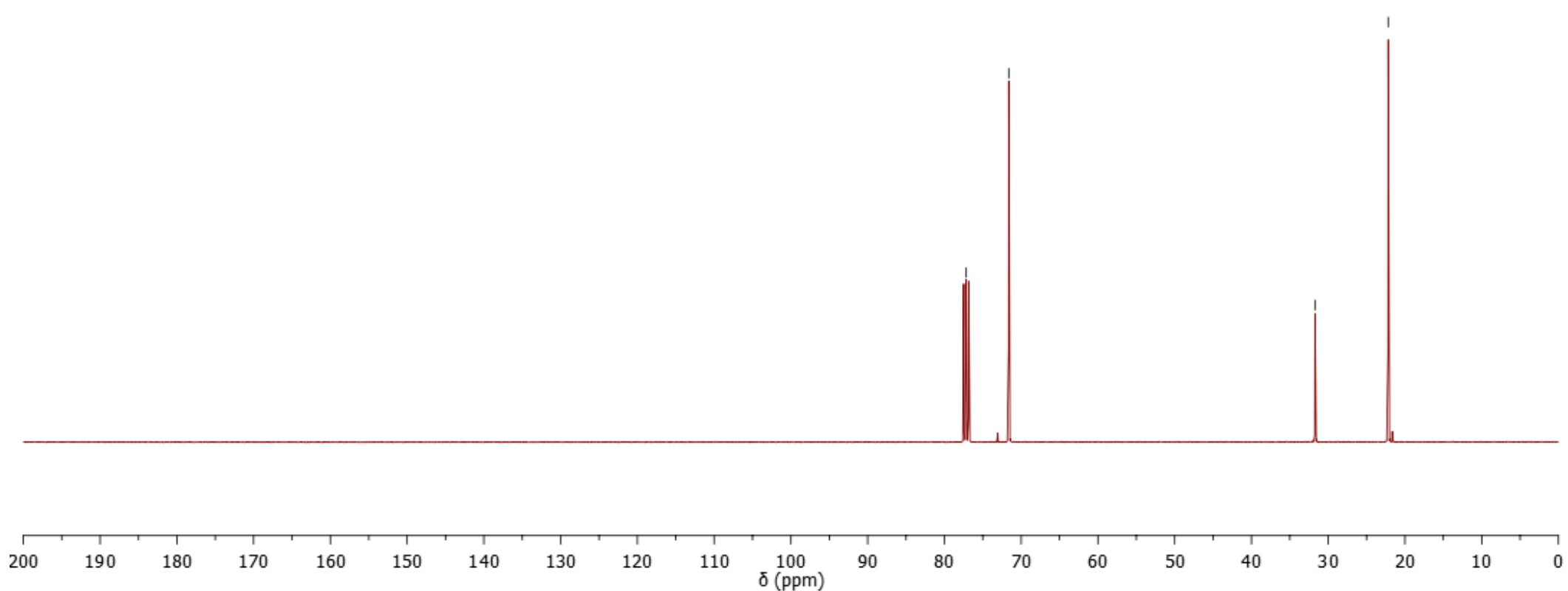
$\underline{\text { Thiophene boronate esters }}$

${ }^{1} \mathrm{H} 400 \mathrm{MHz}, \mathrm{CDCl}_{3}$

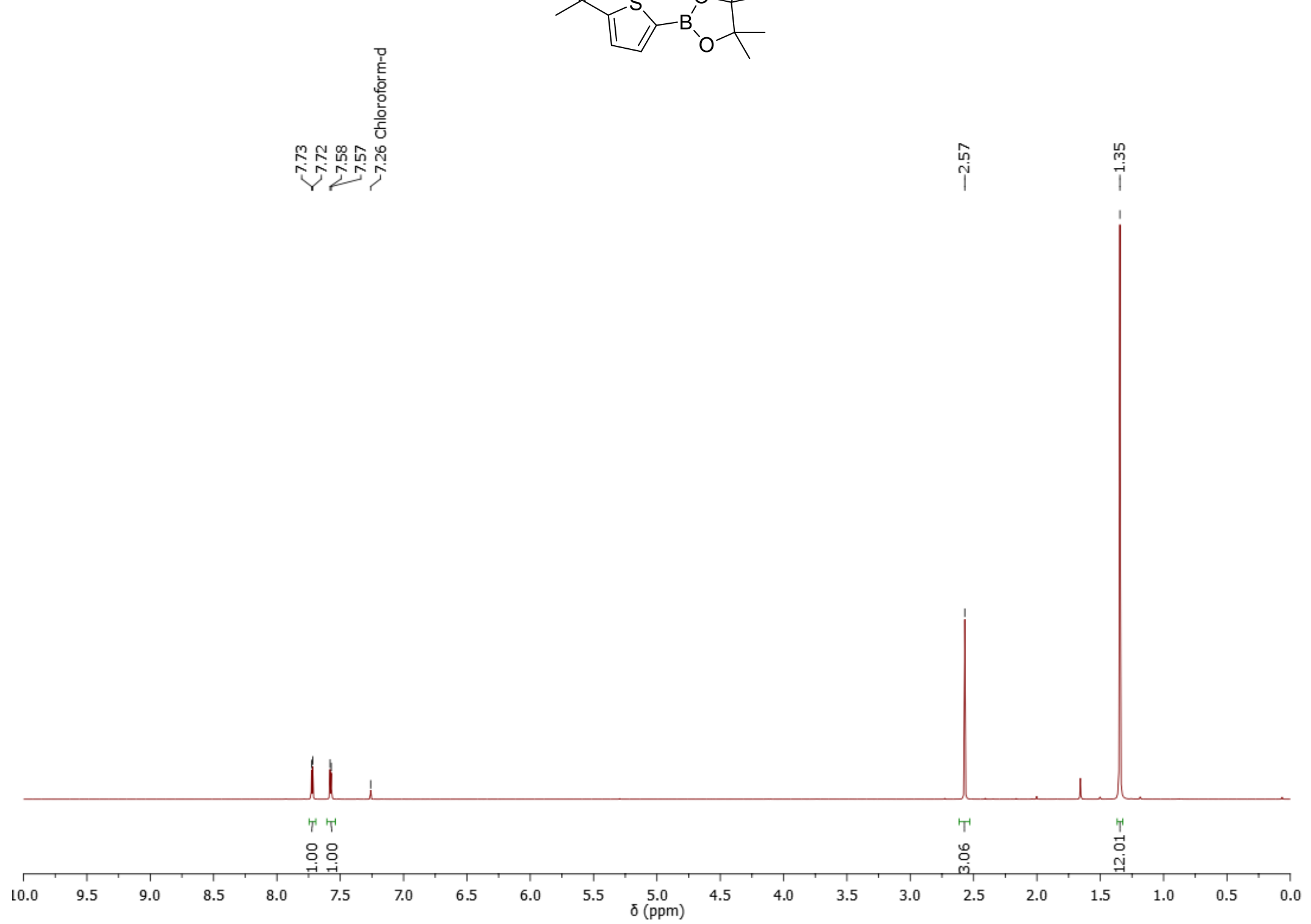


$\underline{\text { Thiophene boronate esters }}$

${ }^{13} \mathrm{C} 101 \mathrm{MHz}$

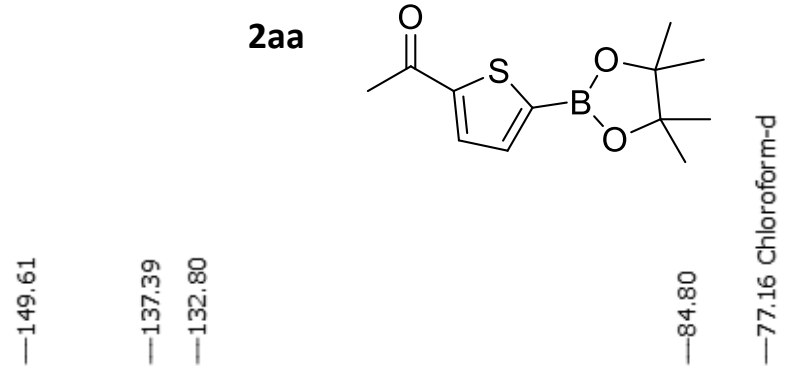

ஸ̃

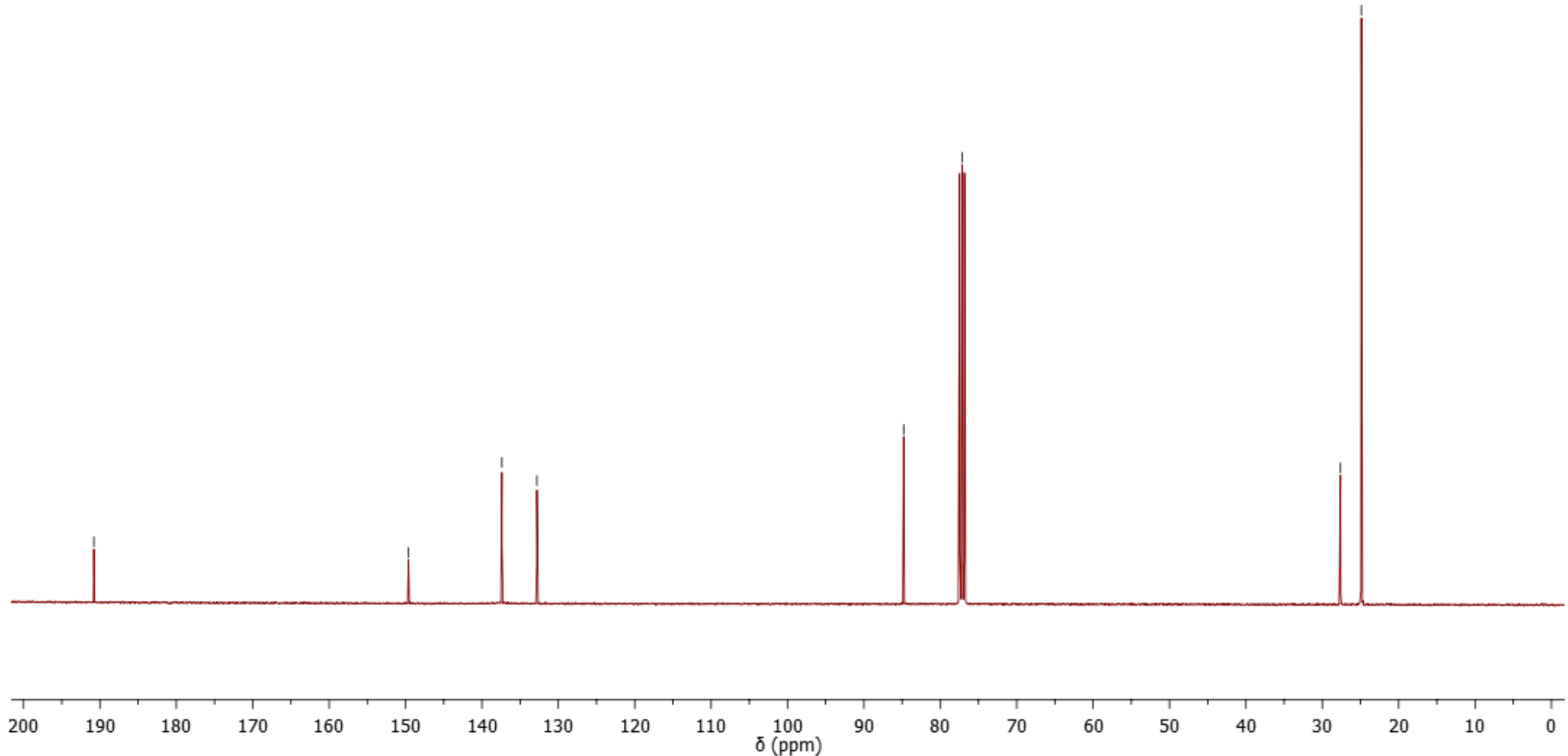


Thiophene boronate esters

${ }^{1} \mathrm{H} 400 \mathrm{MHz}, \mathrm{CDCl}_{3}$

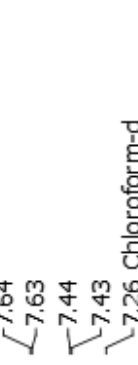

$2 a b$

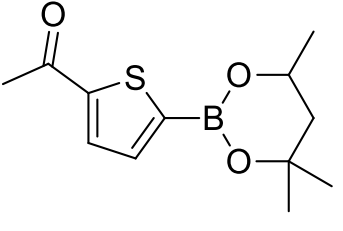

mै

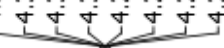

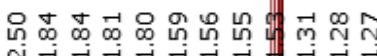
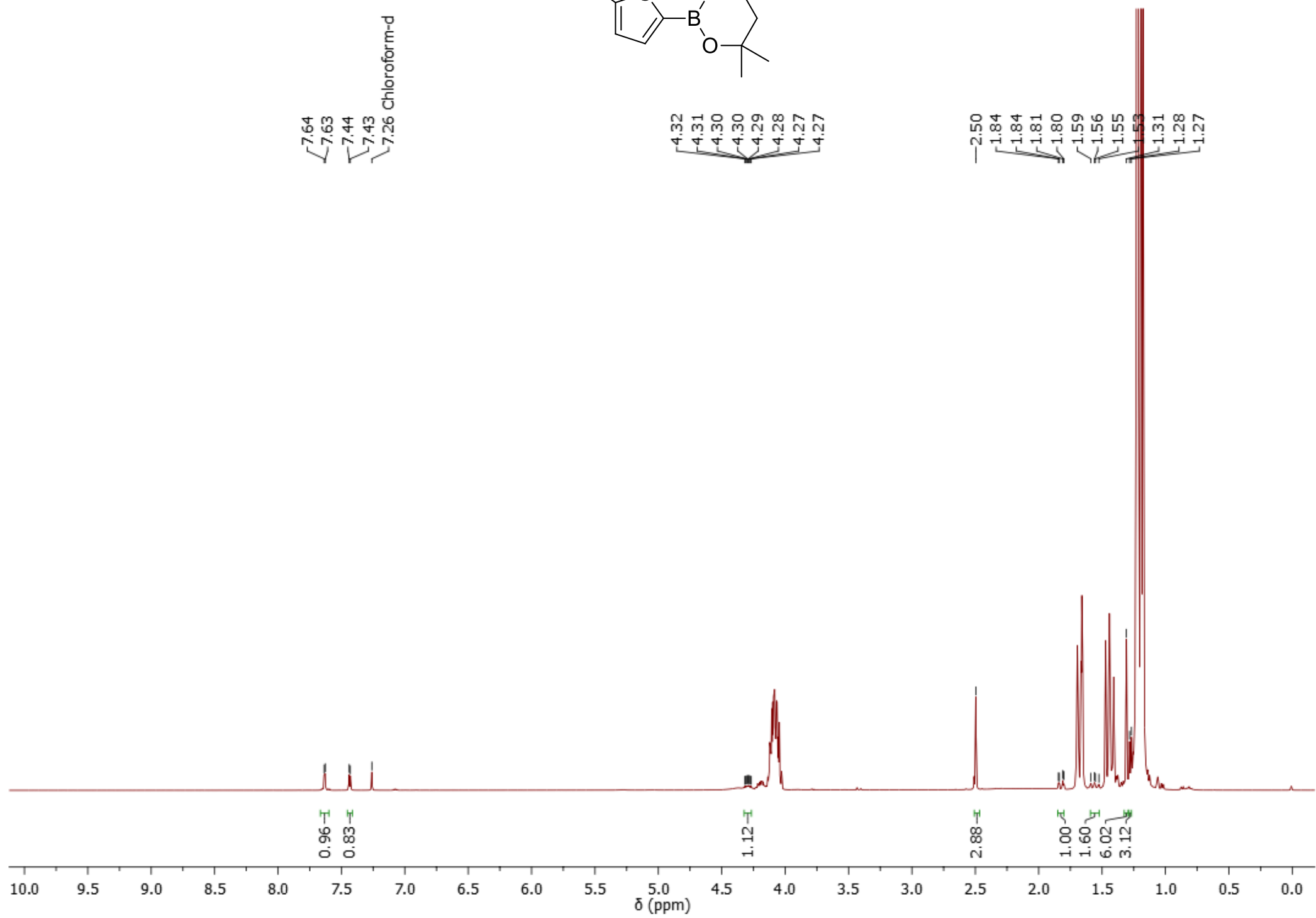
$\underline{\text { Thiophene boronate esters }}$

${ }^{13} \mathrm{C} 101 \mathrm{MHz}$

$2 a b$

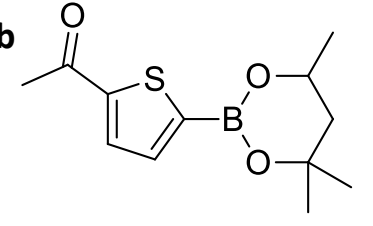

할

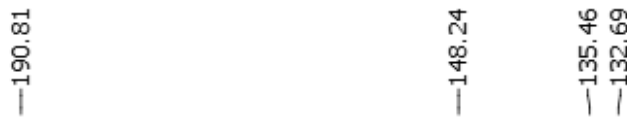

눙요

กำ

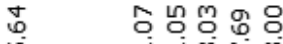

ए min

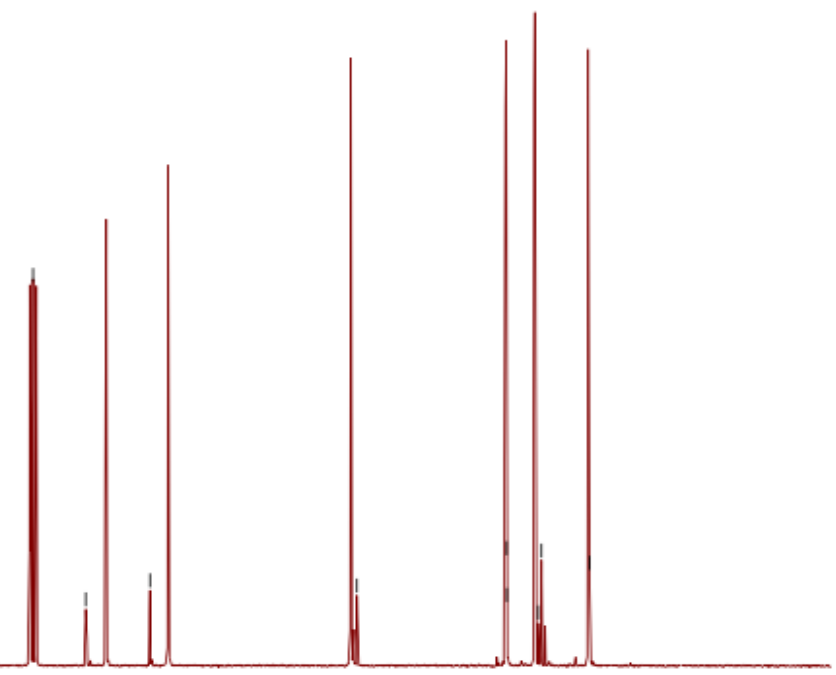


$\underline{\text { Thiophene boronate esters }}$

${ }^{1} \mathrm{H} 400 \mathrm{MHz}, \mathrm{CDCl}_{3}$

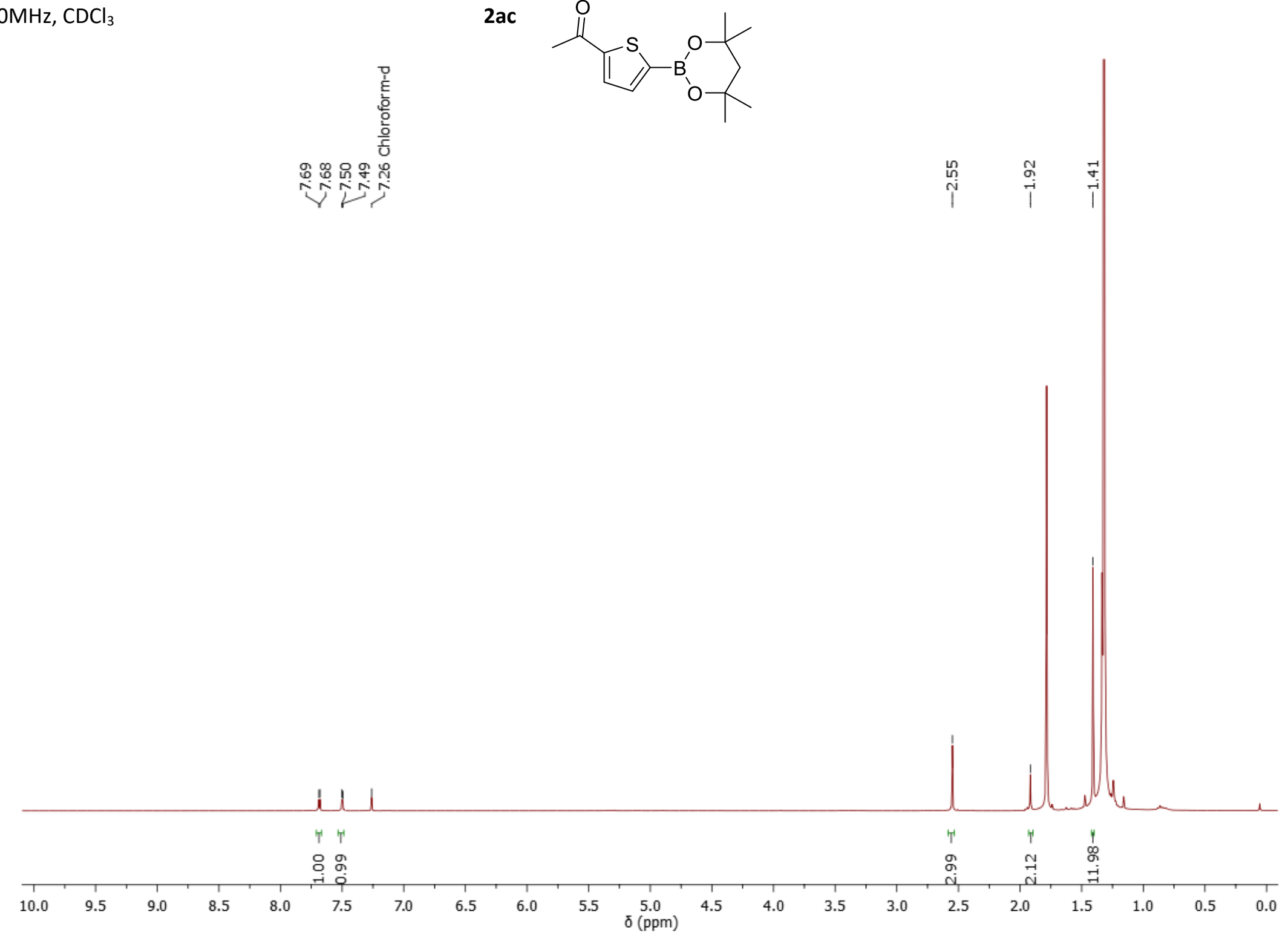


$\underline{\text { Thiophene boronate esters }}$

${ }^{13} \mathrm{C} 101 \mathrm{MHz}$

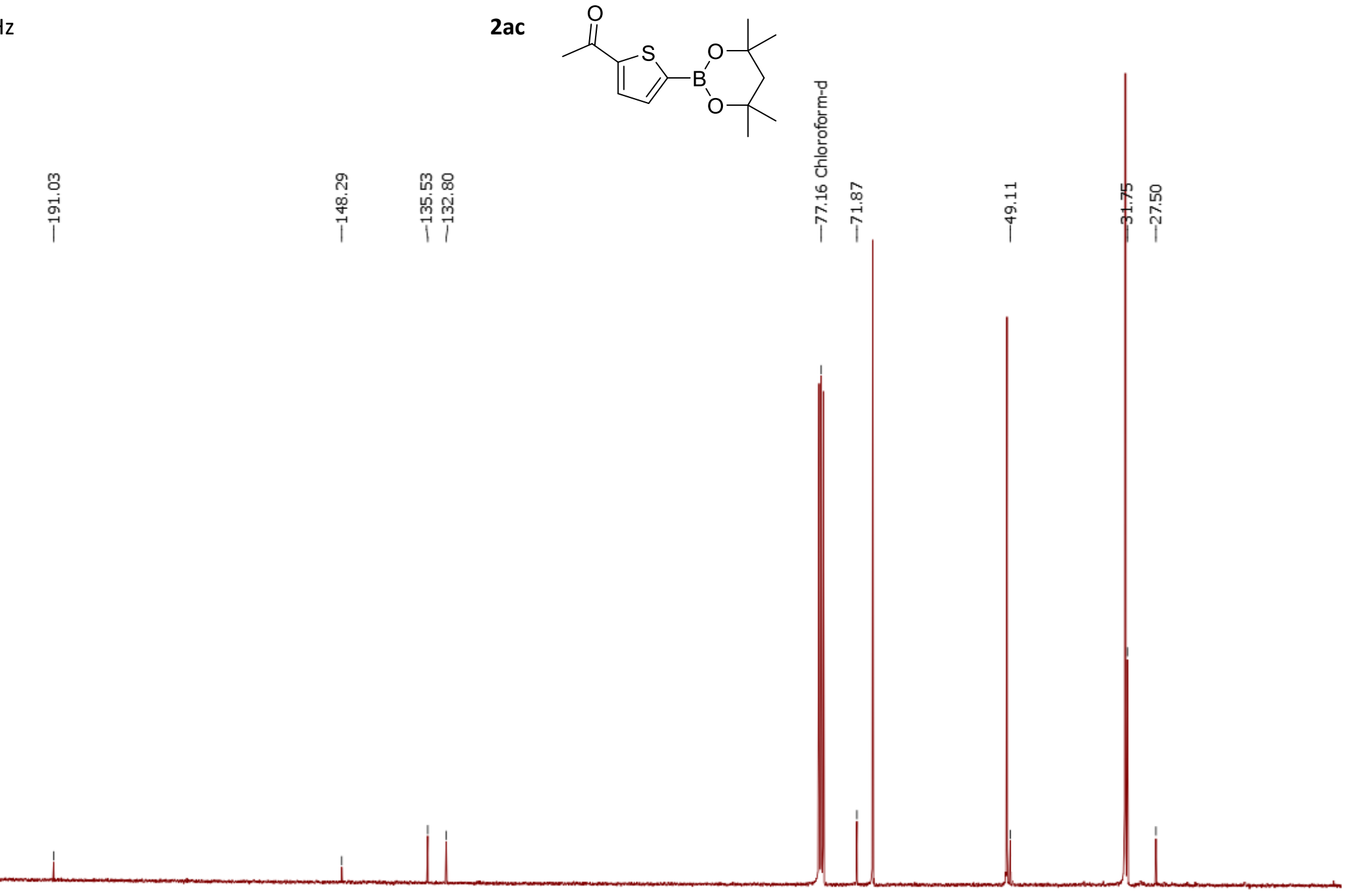$$
200
$$

$190 \quad 180 \quad 170 \quad 160$

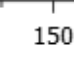

140

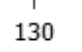

120

$10 \quad \begin{aligned} & 100 \\ & \delta(\mathrm{ppm})\end{aligned}$
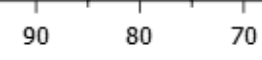

60

50
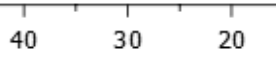
$\underline{\text { Thiophene boronate esters }}$

${ }^{1} \mathrm{H} 400 \mathrm{MHz}, \mathrm{CDCl}_{3}$

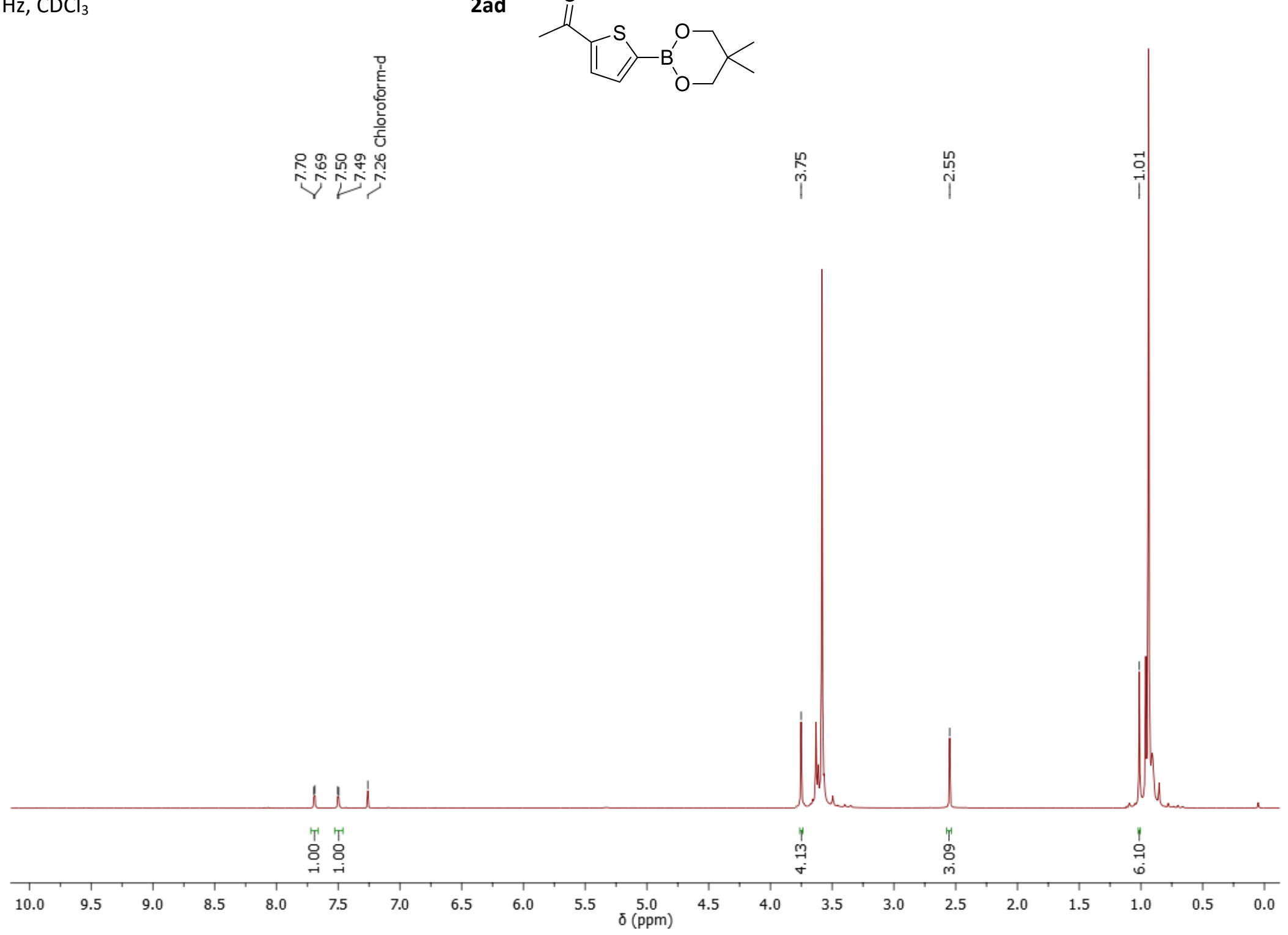


$\underline{\text { Thiophene boronate esters }}$

${ }^{13} \mathrm{C} 101 \mathrm{MHz}$
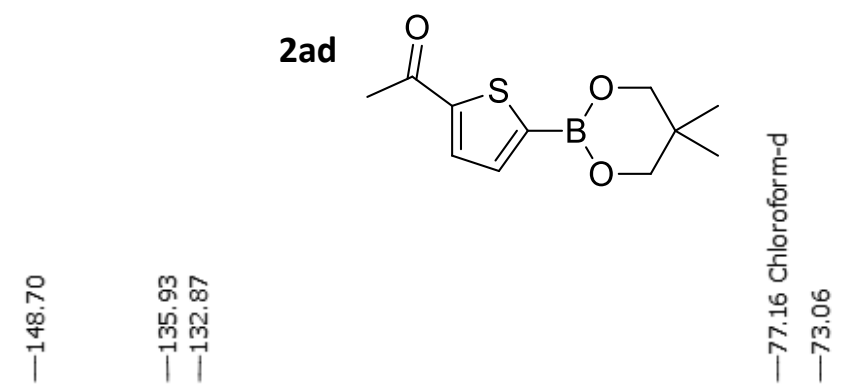

$\stackrel{m}{g} g$

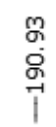

।

गंग
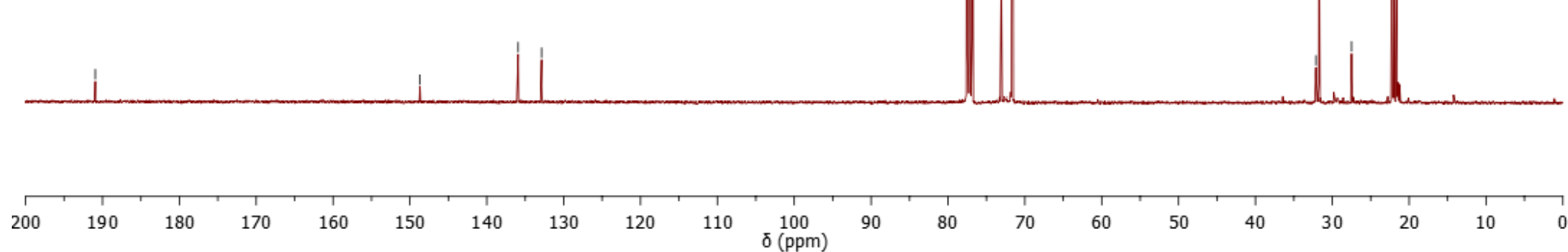
$\underline{\text { Thiophene boronate esters }}$

${ }^{1} \mathrm{H} 400 \mathrm{MHz}, \mathrm{CDCl}_{3}$

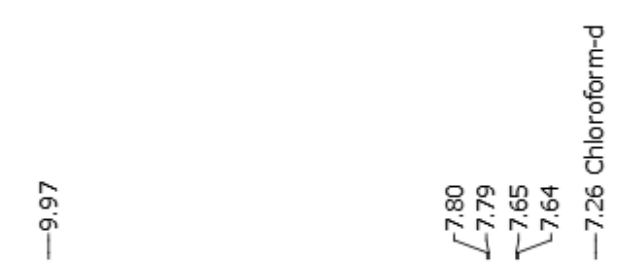
㓡点
2 ba

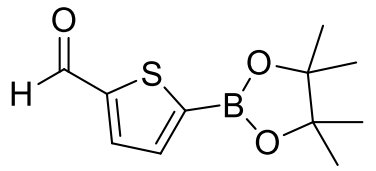

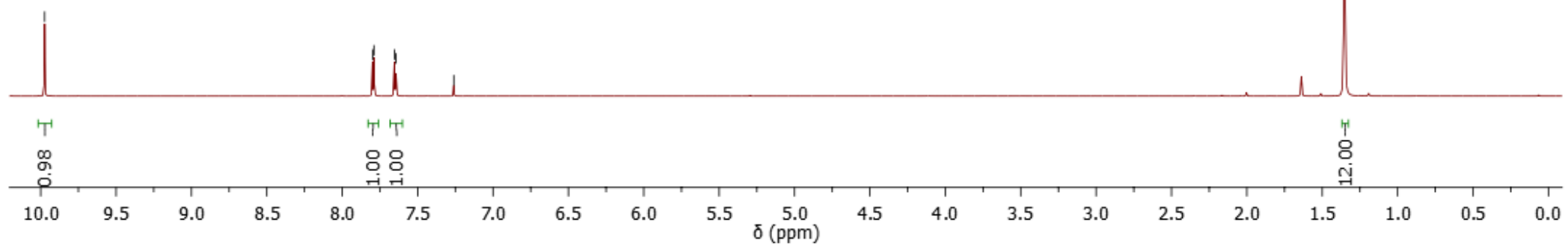


$\underline{\text { Thiophene boronate esters }}$

${ }^{13} \mathrm{C} 101 \mathrm{MHz}$

$2 \mathrm{ba}$

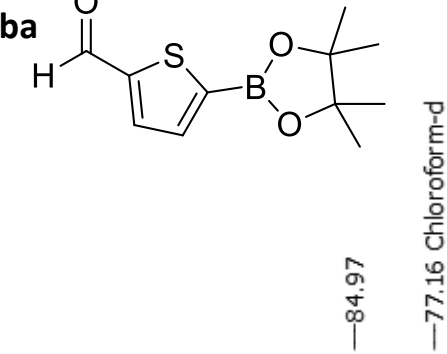

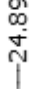

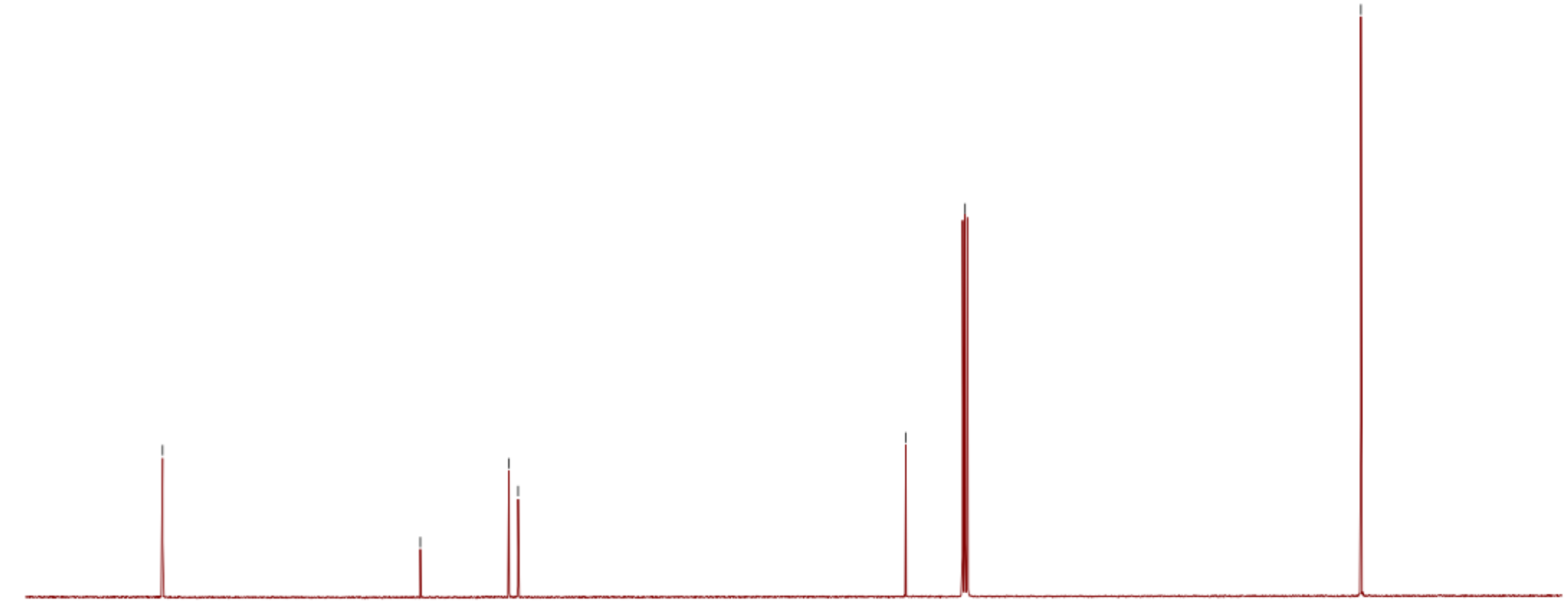

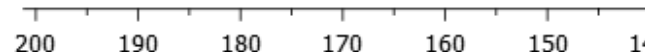

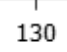

120

$\begin{array}{cc}110 & 100 \\ \delta(\mathrm{ppm})\end{array}$

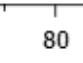

70 
$\underline{\text { Thiophene boronate esters }}$

${ }^{1} \mathrm{H} 400 \mathrm{MHz}, \mathrm{CDCl}_{3}$

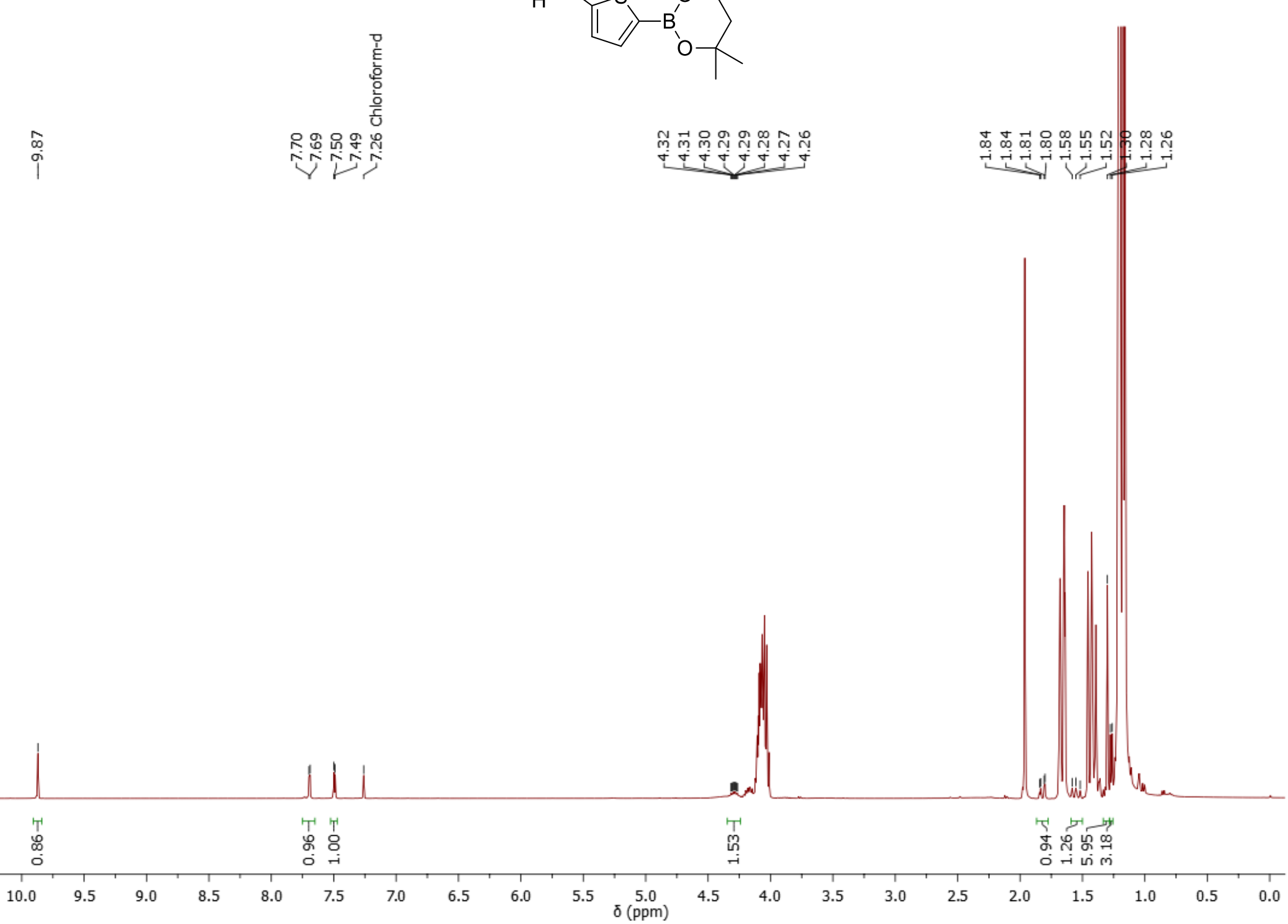


$\underline{\text { Thiophene boronate esters }}$

${ }^{13} \mathrm{C} 101 \mathrm{MHz}$

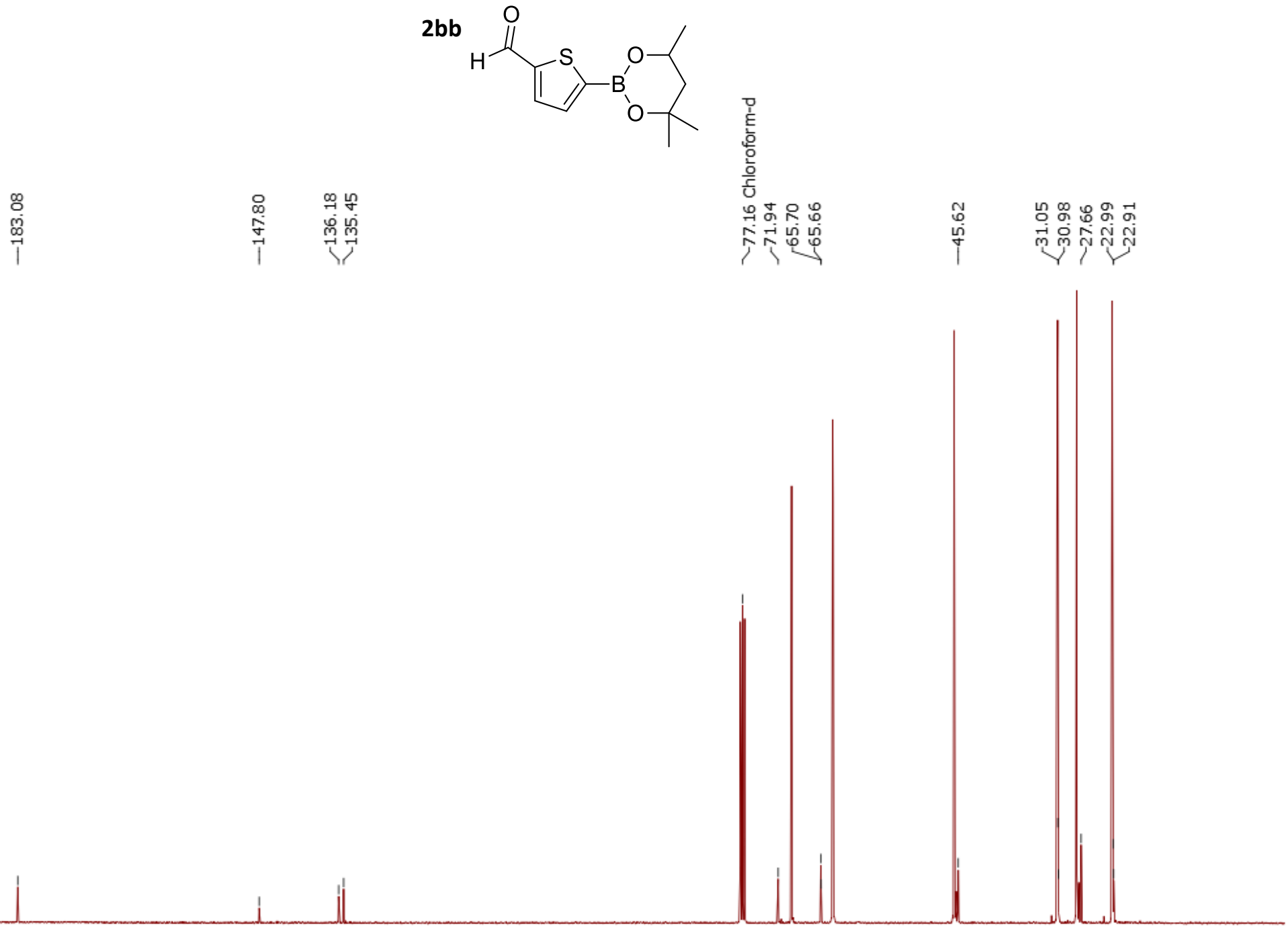


$\underline{\text { Thiophene boronate esters }}$

${ }^{1} \mathrm{H} 400 \mathrm{MHz}, \mathrm{CDCl}_{3}$

每

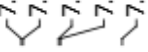

$2 b c$

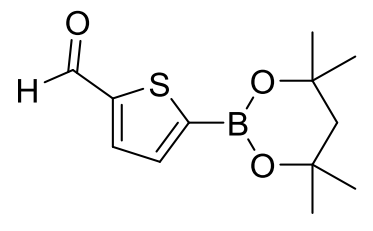

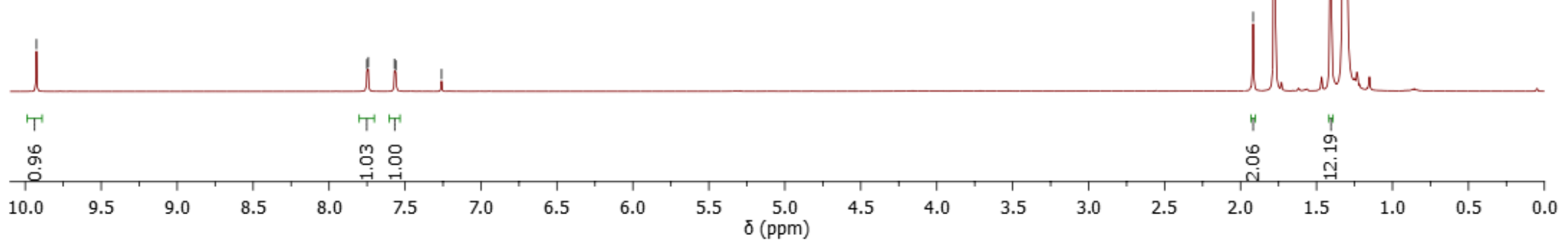


$\underline{\text { Thiophene boronate esters }}$

${ }^{13} \mathrm{C} 101 \mathrm{MHz}$

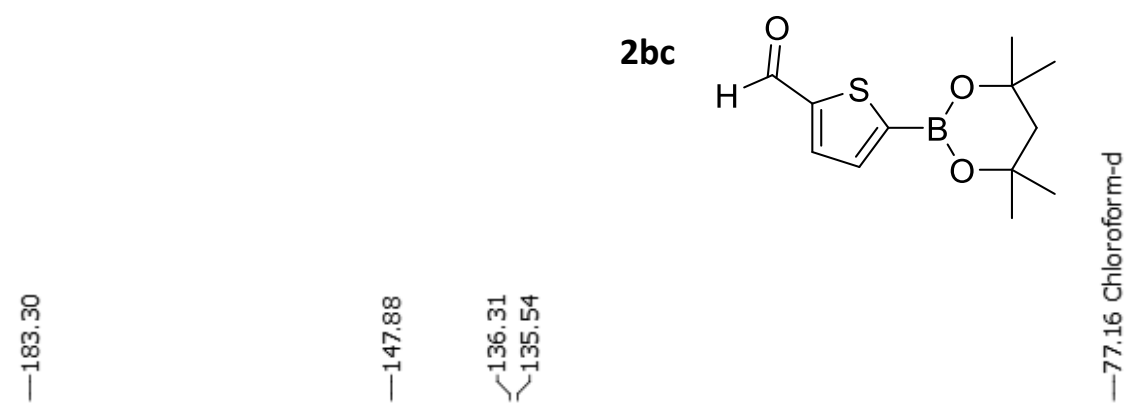

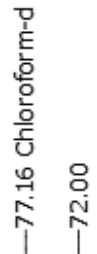

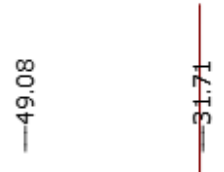

$200 \quad 190$ 
$\underline{\text { Thiophene boronate esters }}$

${ }^{1} \mathrm{H} 400 \mathrm{MHz}, \mathrm{CDCl}_{3}$

i

$\stackrel{\infty}{\stackrel{\infty}{p}}$

$\stackrel{m}{\text { I }}$

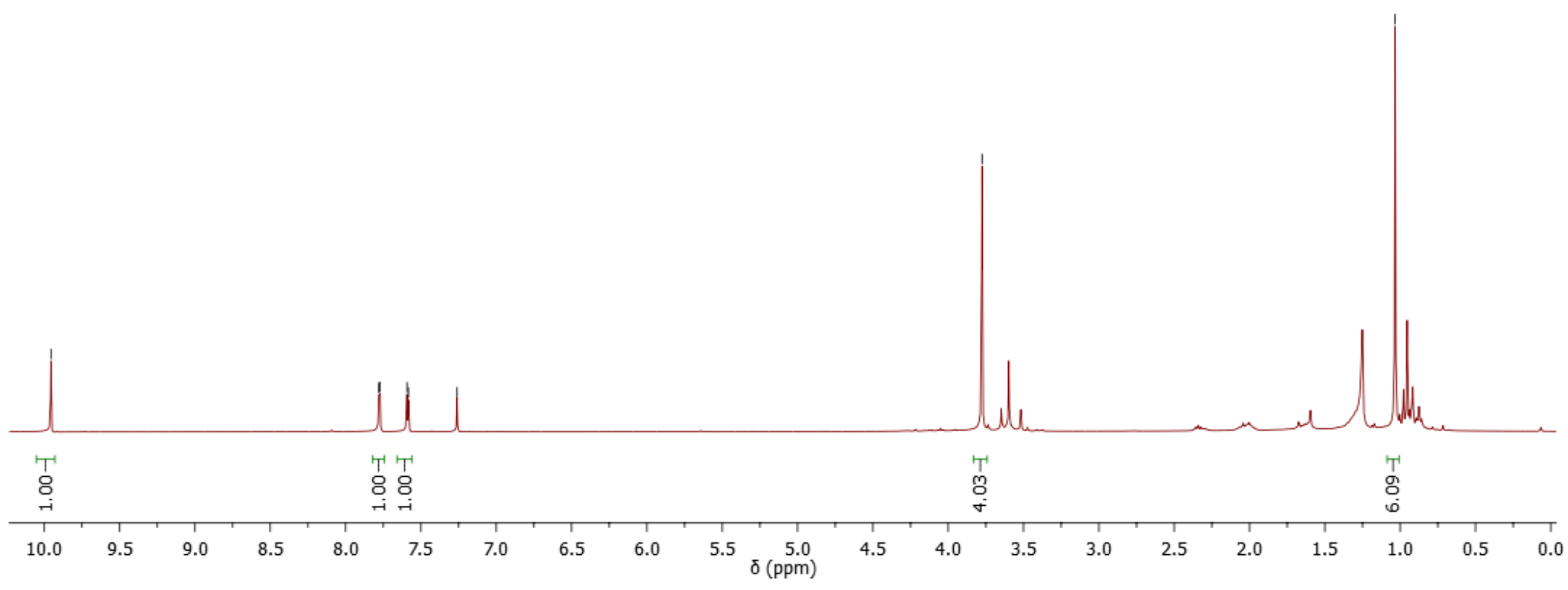


$\underline{\text { Thiophene boronate esters }}$

${ }^{13} \mathrm{C} 101 \mathrm{MHz}$

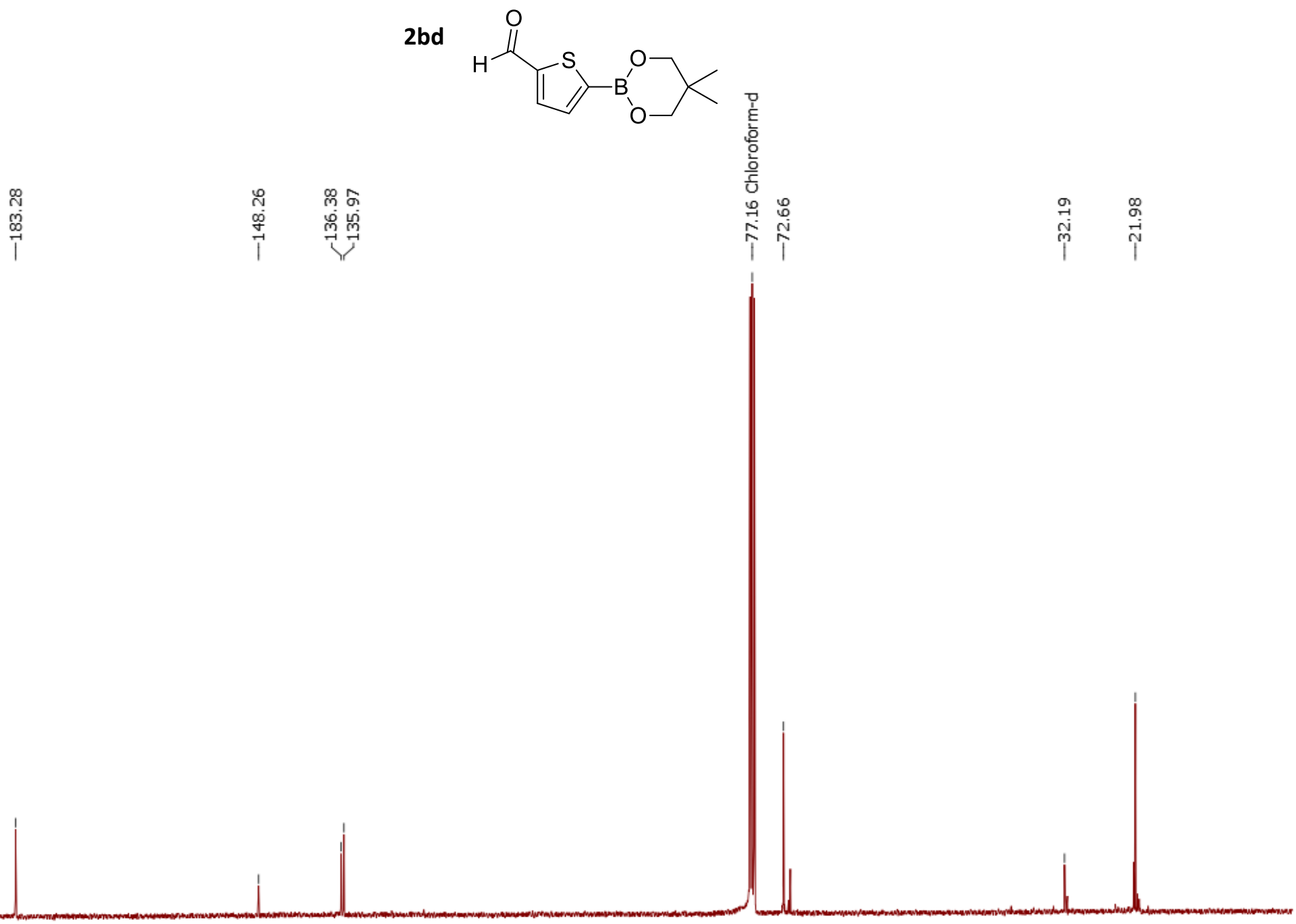


$\underline{\text { Thiophene boronate esters }}$

${ }^{1} \mathrm{H} 400 \mathrm{MHz}, \mathrm{CDCl}_{3}$

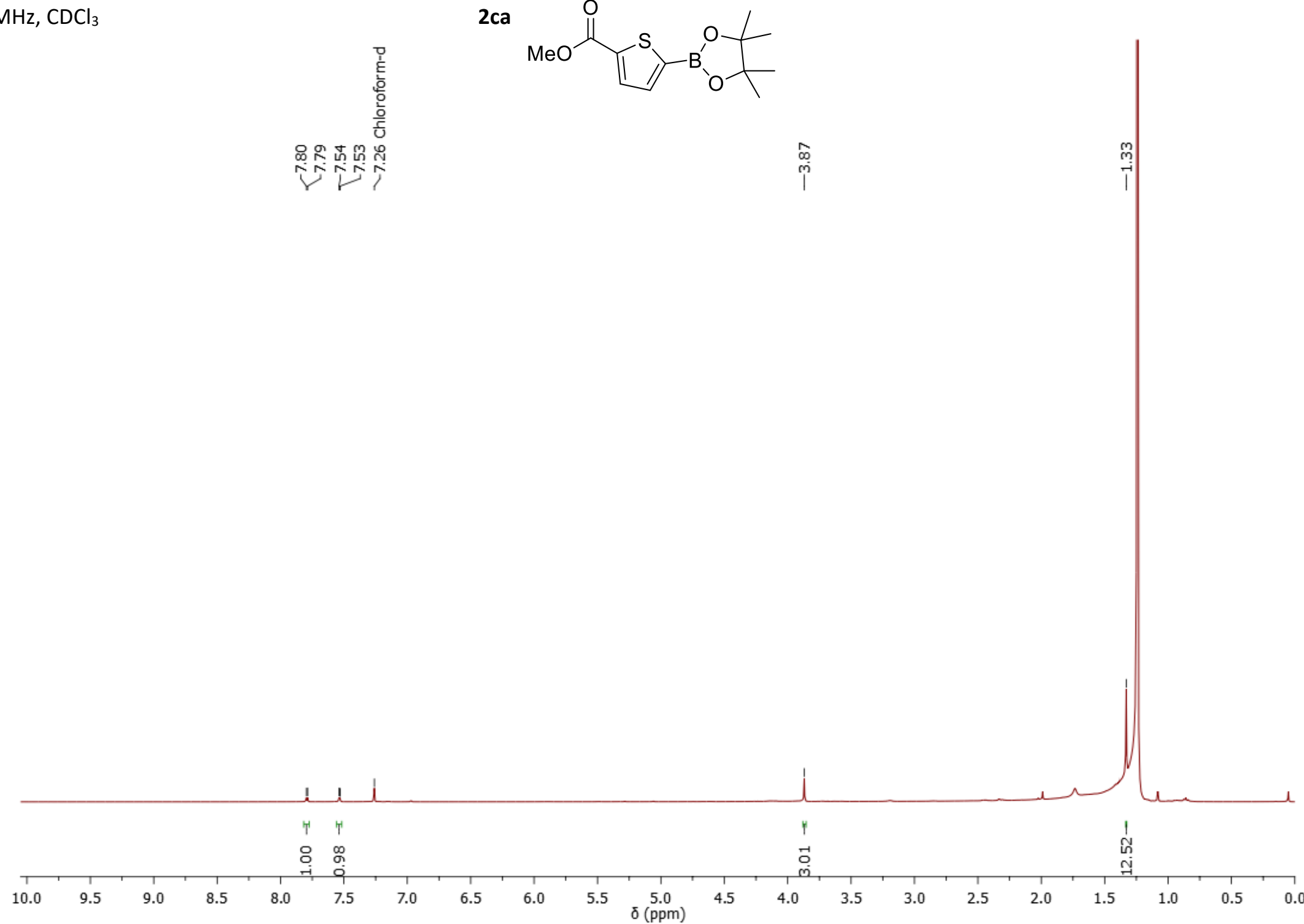


$\underline{\text { Thiophene boronate esters }}$

${ }^{13} \mathrm{C} 101 \mathrm{MHz}, \mathrm{CDCl}_{3}$
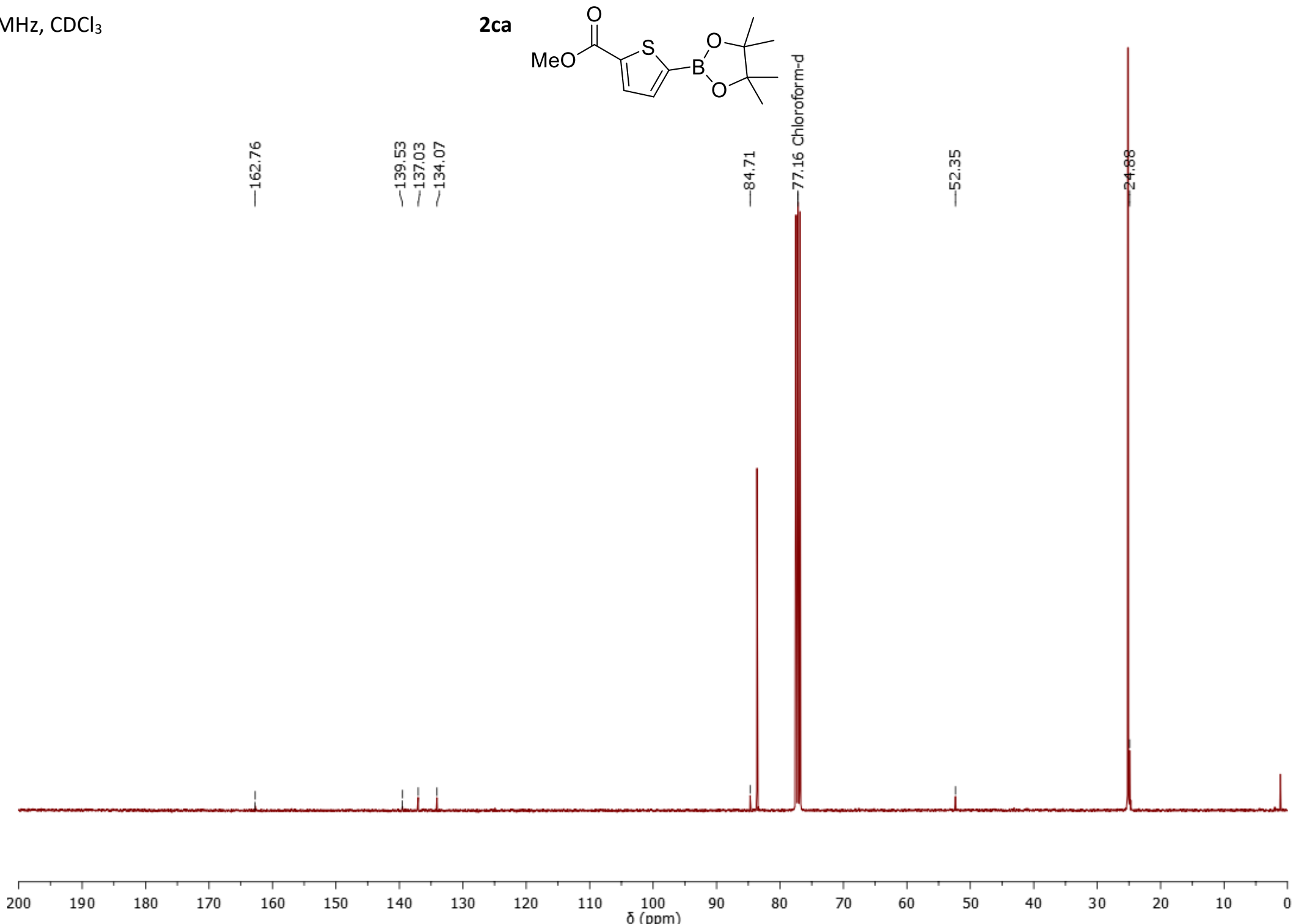
$\underline{\text { Thiophene boronate esters }}$

${ }^{1} \mathrm{H} 400 \mathrm{MHz}, \mathrm{CDCl}_{3}$

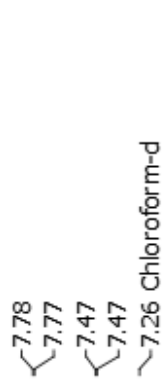

$2 c b$

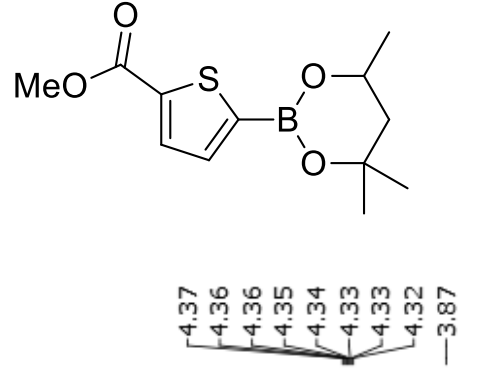

我

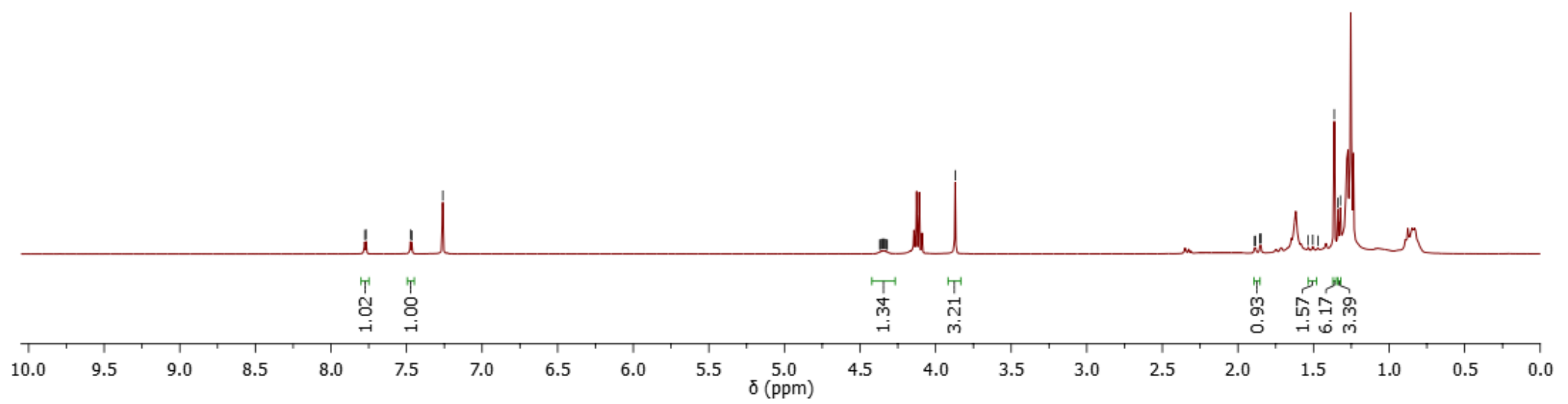


Thiophene boronate esters

${ }^{13} \mathrm{C} 101 \mathrm{MHz}$
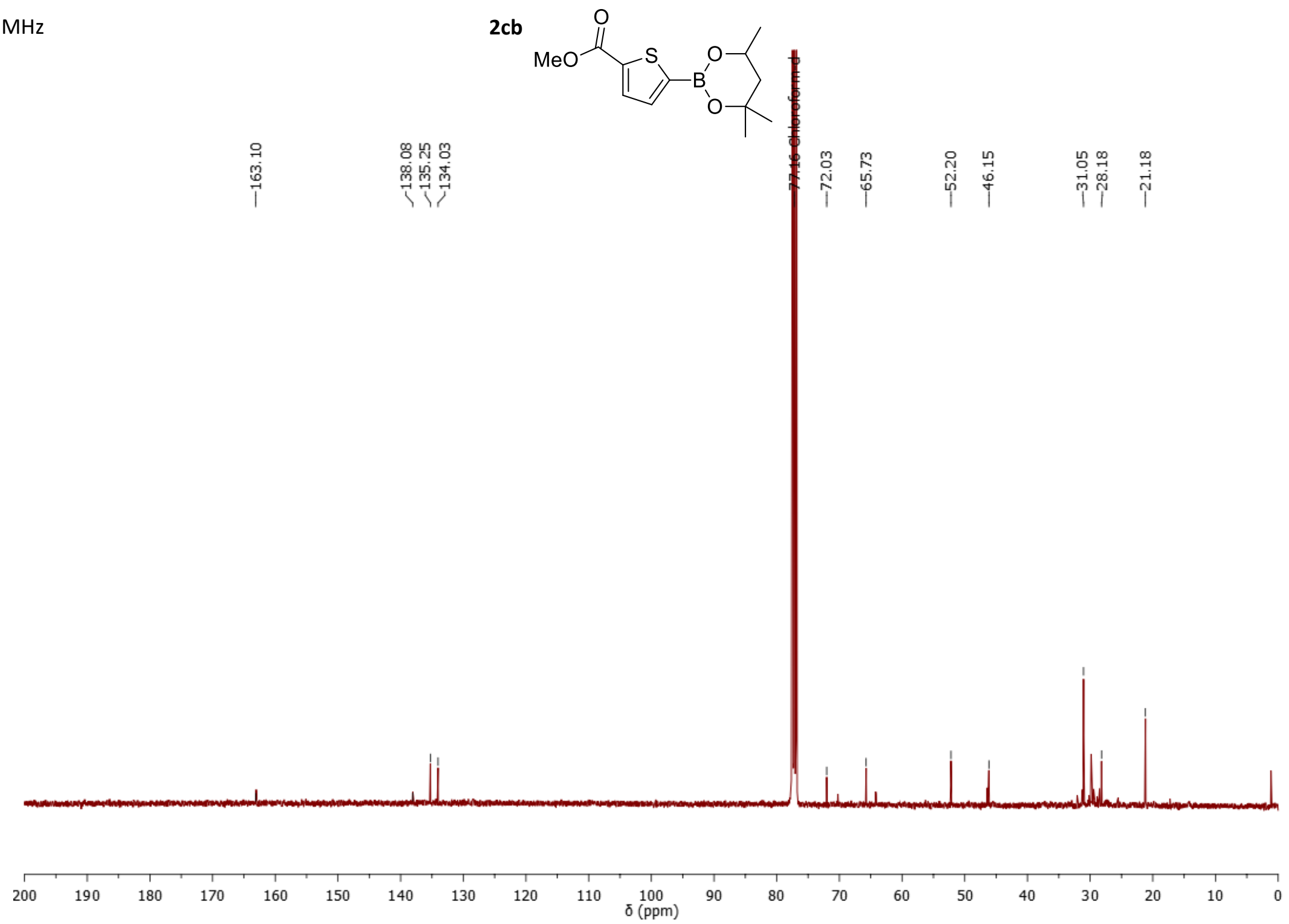

S72 
$\underline{\text { Thiophene boronate esters }}$

${ }^{1} \mathrm{H} 400 \mathrm{MHz}, \mathrm{CDCl}_{3}$

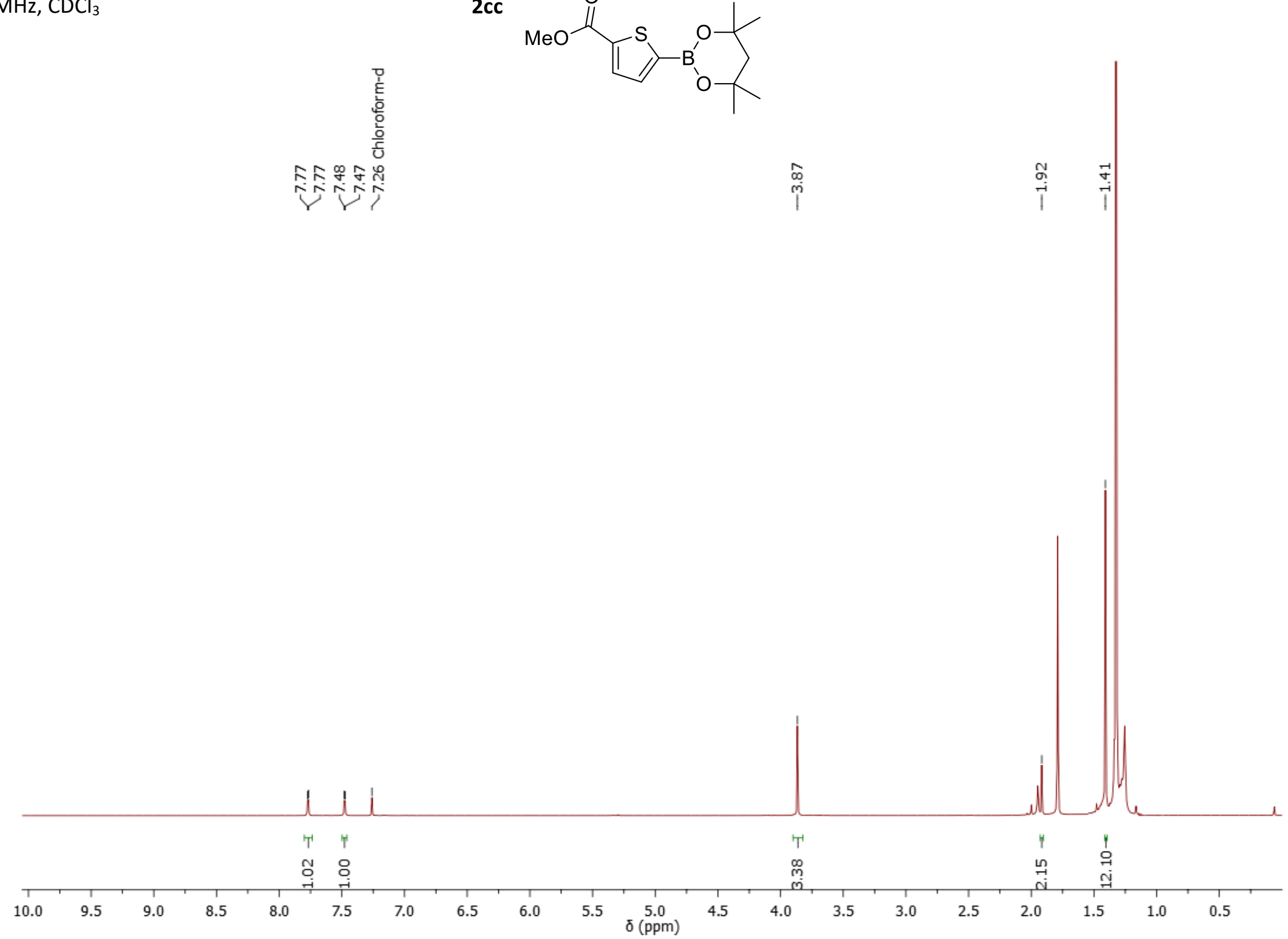


$\underline{\text { Thiophene boronate esters }}$

${ }^{13} \mathrm{C} 101 \mathrm{MHz}$
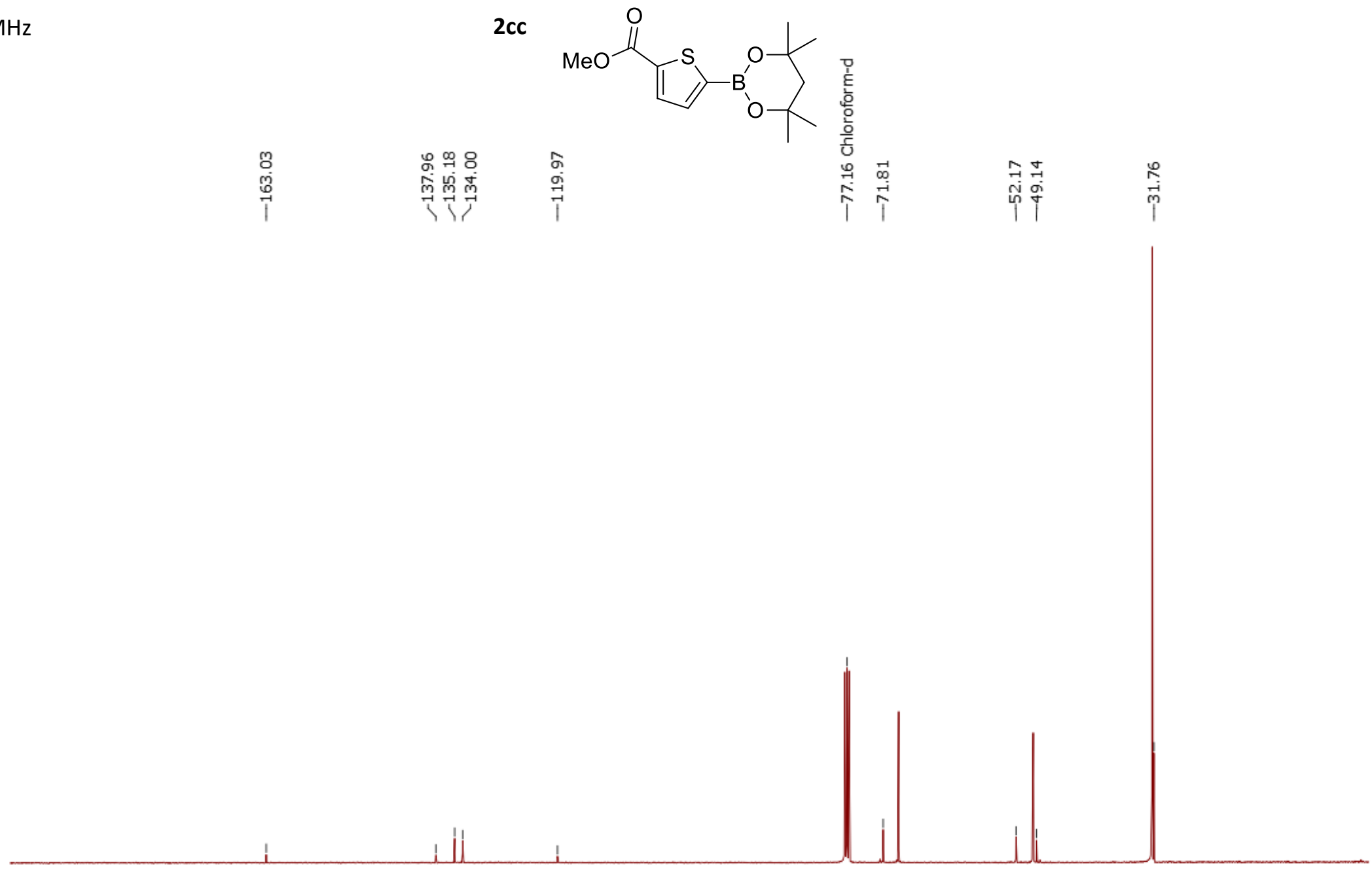
$\underline{\text { Thiophene boronate esters }}$

${ }^{1} \mathrm{H} 400 \mathrm{MHz}, \mathrm{CDCl}_{3}$

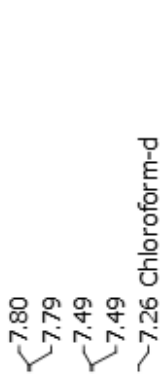

2cd

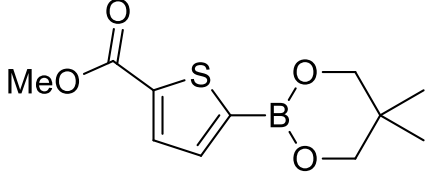

$\stackrel{\infty}{\infty}$

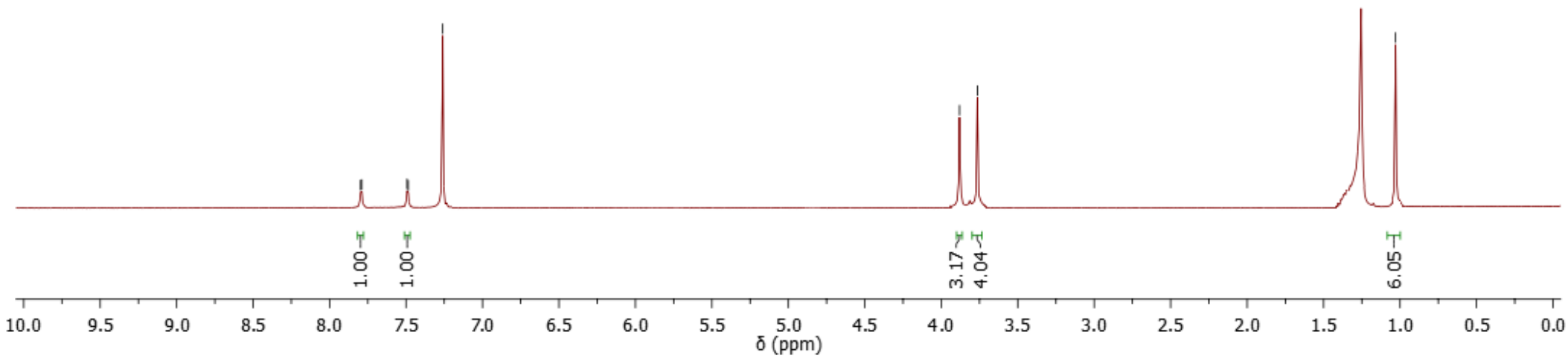


$\underline{\text { Thiophene boronate esters }}$

${ }^{13} \mathrm{C} 101 \mathrm{MHz}$

$$
\text { 2cd }
$$

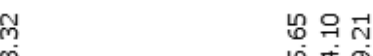

ஸे

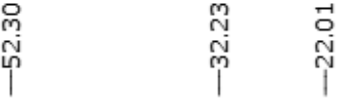

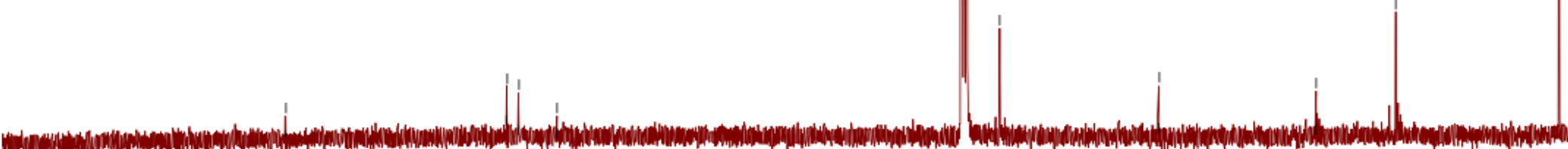$$
200
$$

190

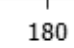

170

160

140

130

120

110

$\delta \stackrel{100}{\delta(\mathrm{ppm})}$

1
$90 \quad 80$

60

50

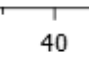

30

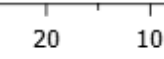


$\underline{\text { Thiophene boronate esters }}$

${ }^{1} \mathrm{H} 400 \mathrm{MHz}, \mathrm{CDCl}_{3}$

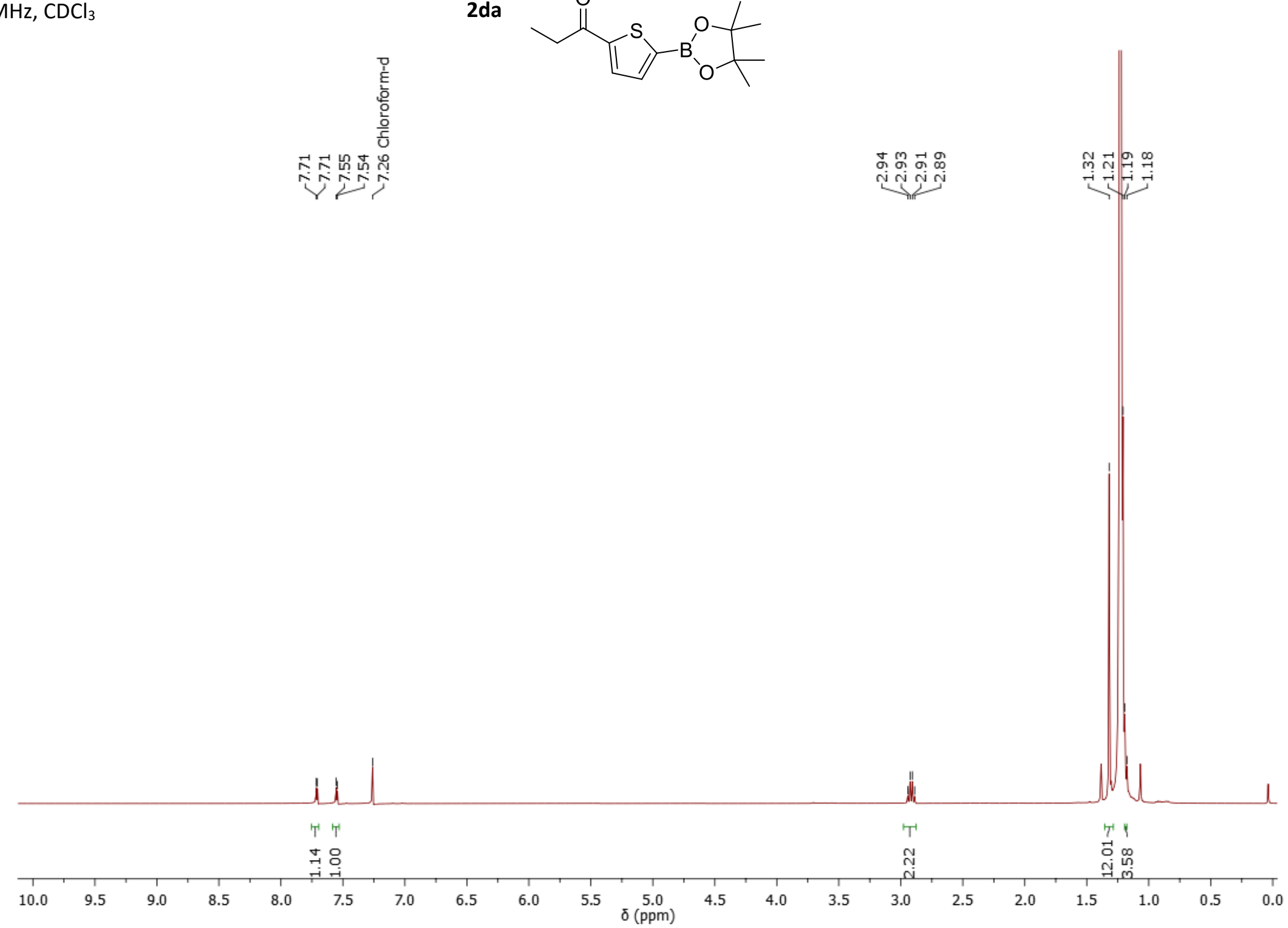


$\underline{\text { Thiophene boronate esters }}$

${ }^{13} \mathrm{C} 101 \mathrm{MHz}$

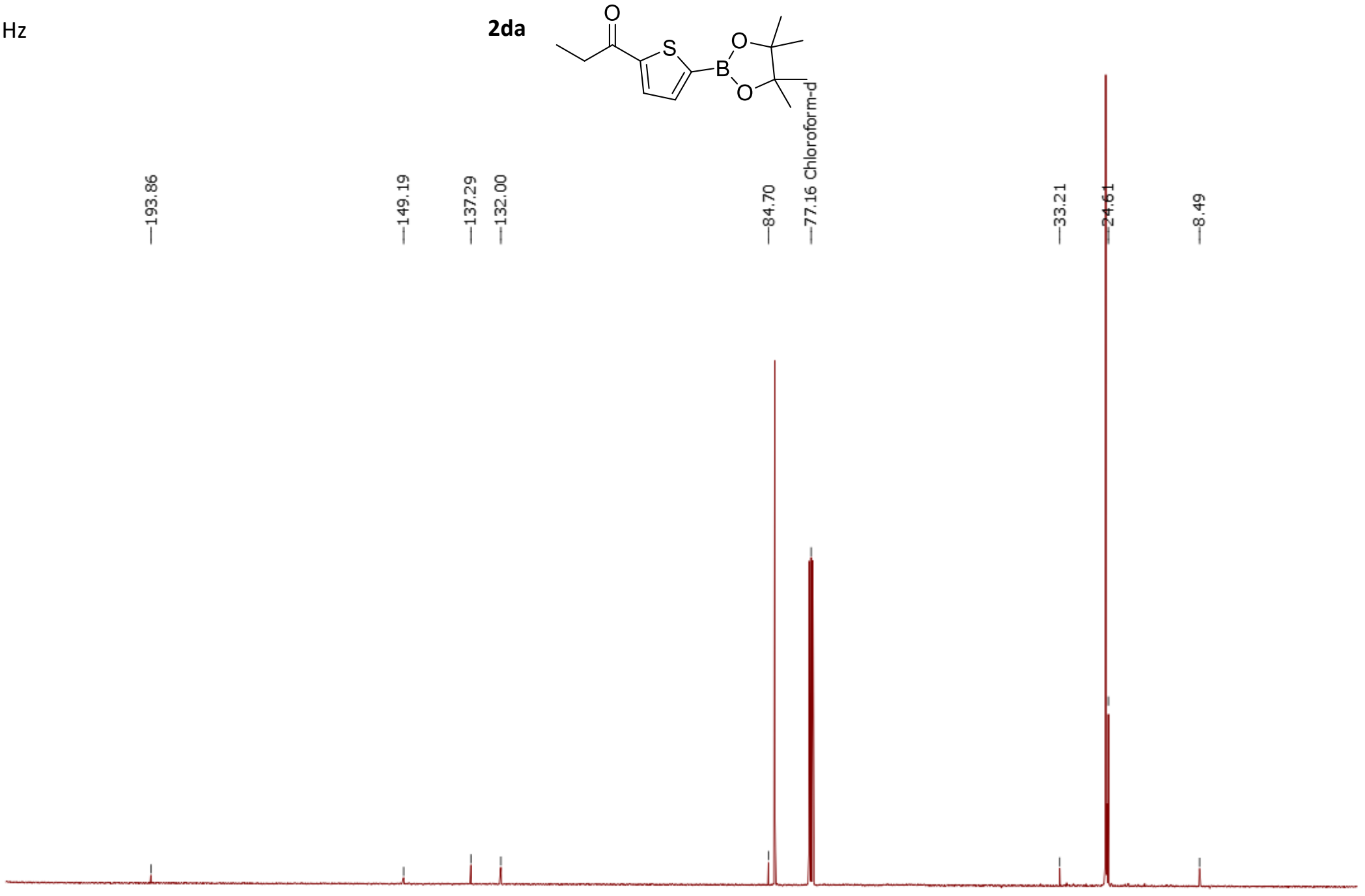

$\begin{array}{llllllllllll}210 & 200 & 190 & 180 & 170 & 160 & 150 & 140 & 130 & 120 & 110 & 100\end{array}$ $\delta(\mathrm{ppm})$ 
$\underline{\text { Thiophene boronate esters }}$

${ }^{1} \mathrm{H} 400 \mathrm{MHz}, \mathrm{CDCl}_{3}$

$2 \mathrm{db}$

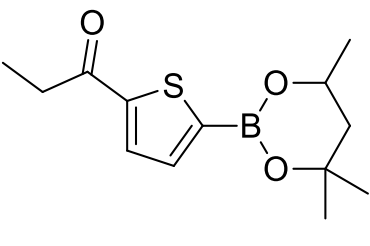

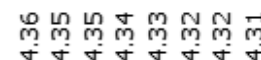

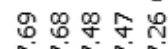

तivi

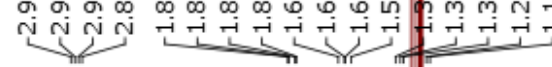

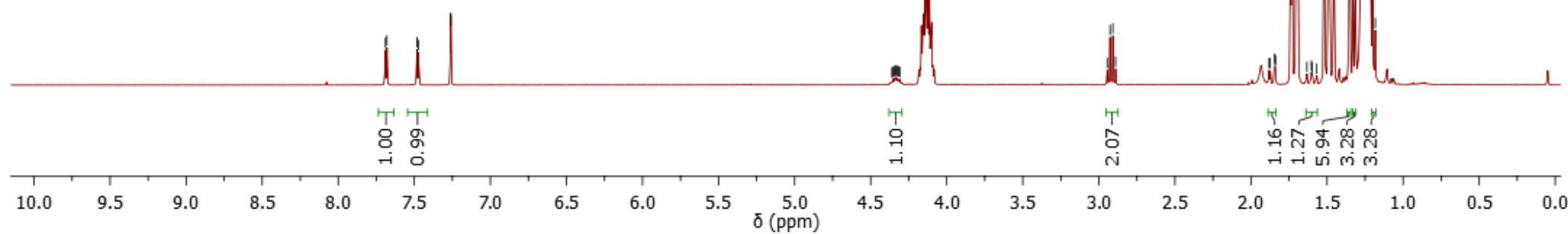


$\underline{\text { Thiophene boronate esters }}$

${ }^{13} \mathrm{C} 101 \mathrm{MHz}$

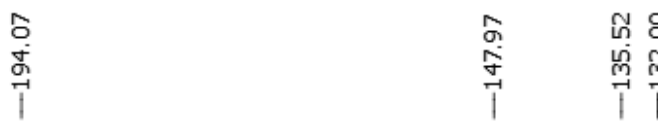

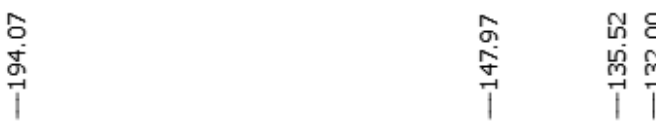

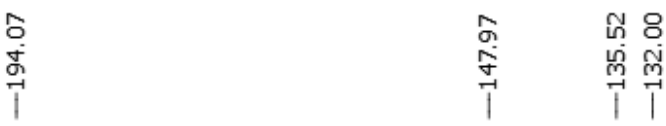

$2 d b$

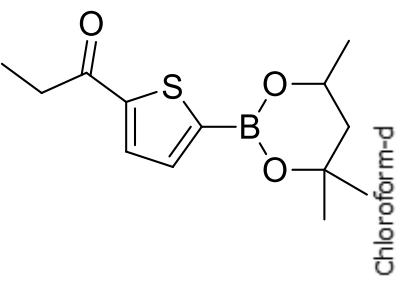

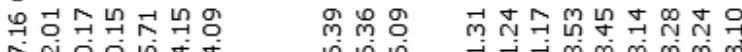

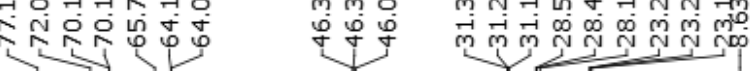

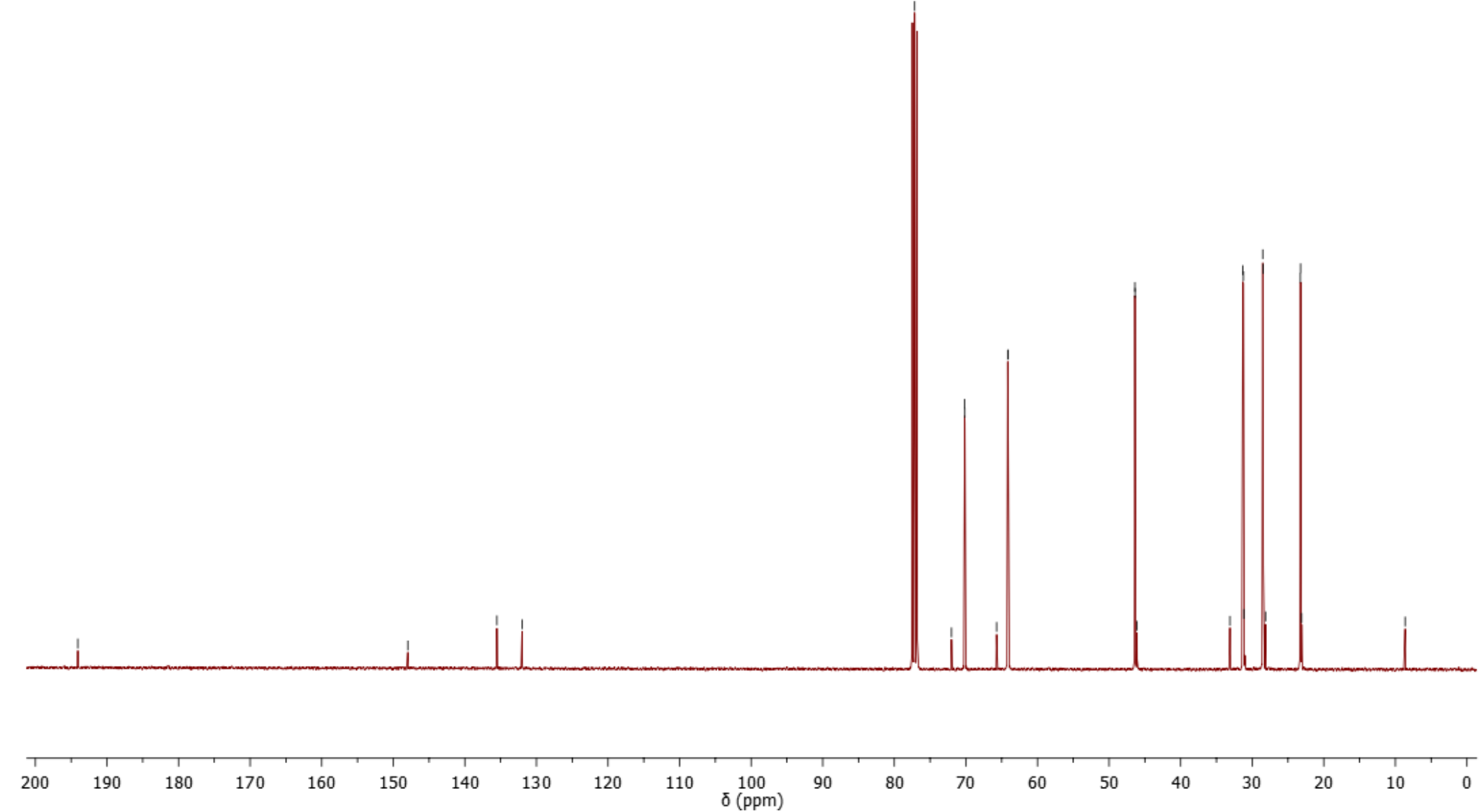


$\underline{\text { Thiophene boronate esters }}$

${ }^{1} \mathrm{H} 400 \mathrm{MHz}, \mathrm{CDCl}_{3}$

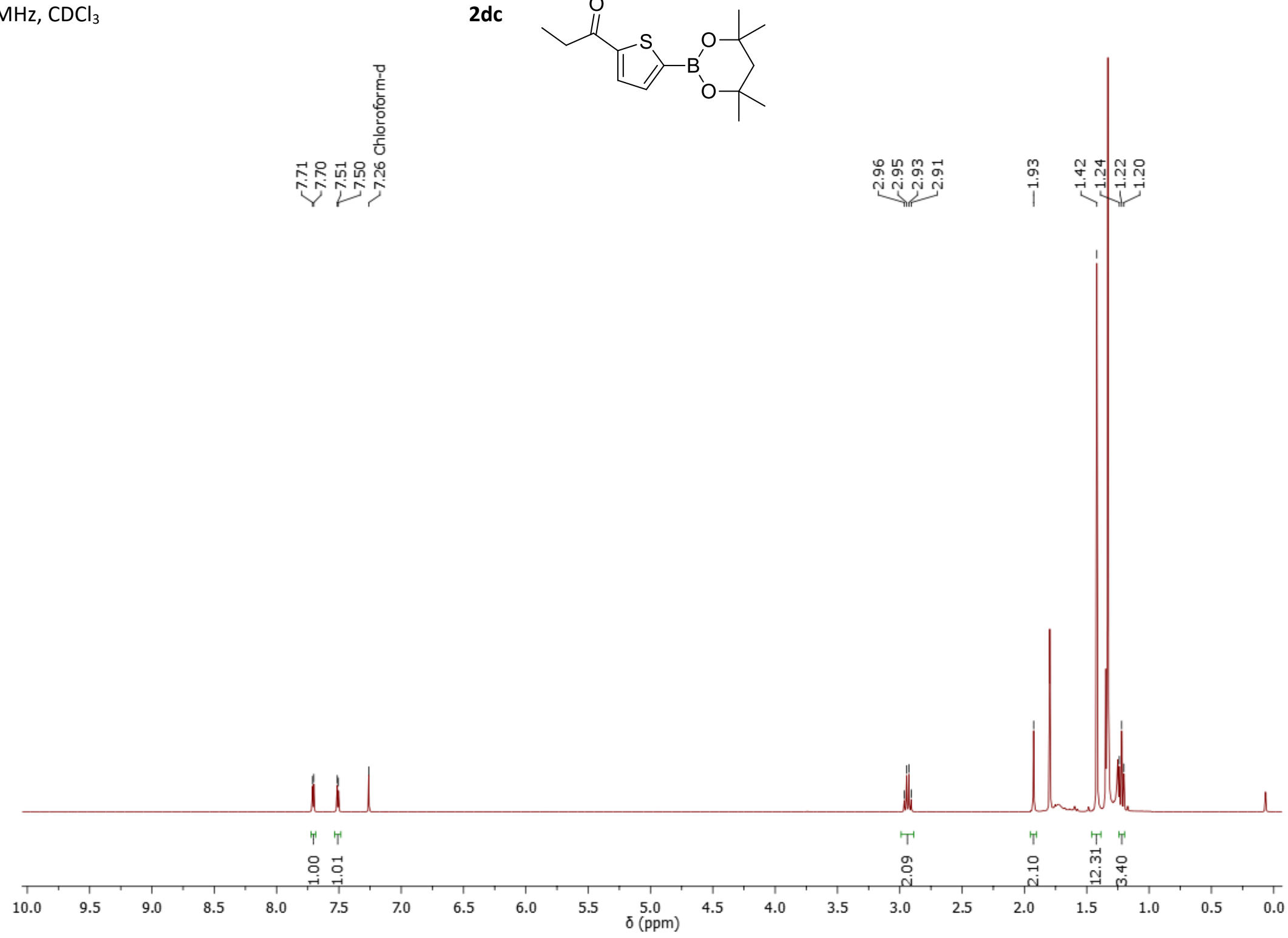


$\underline{\text { Thiophene boronate esters }}$

${ }^{13} \mathrm{C} 101 \mathrm{MHz}$

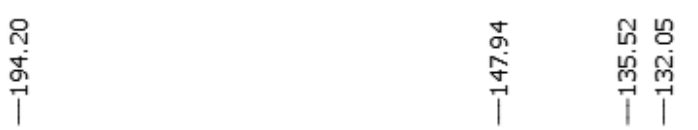

2dc

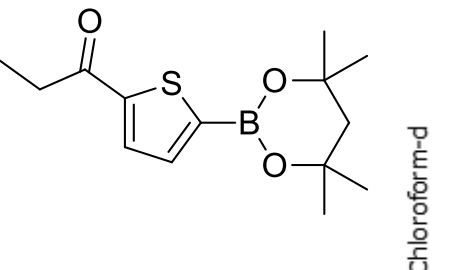

$\frac{1}{5}$
흔
흔

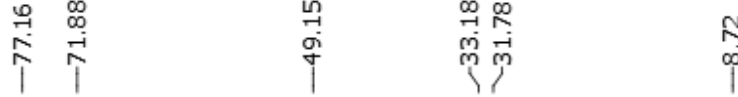

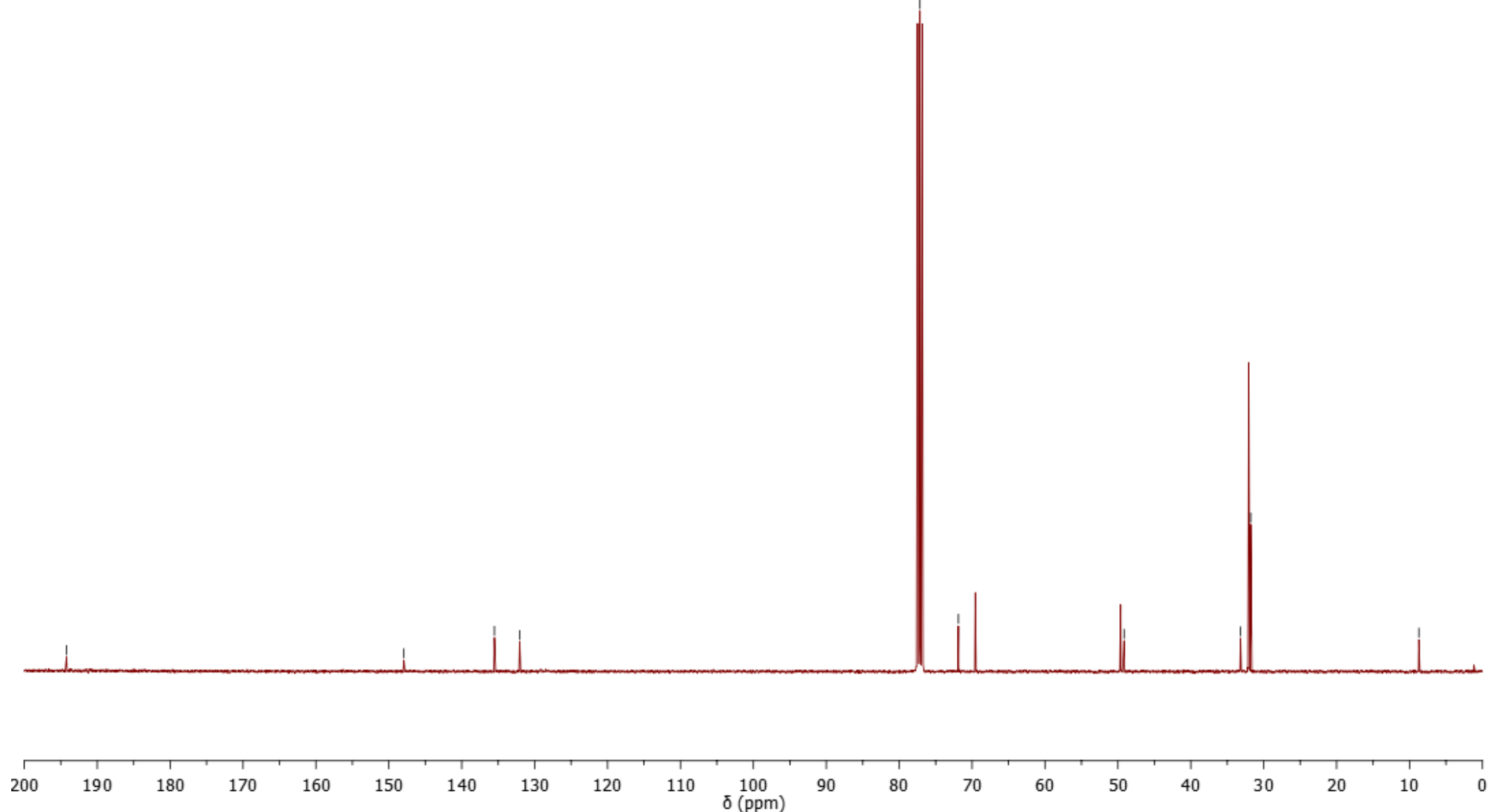


Thiophene boronate esters

${ }^{1} \mathrm{H} 400 \mathrm{MHz}, \mathrm{CDCl}_{3}$

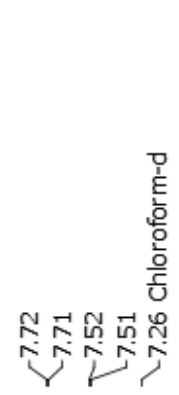

2dd

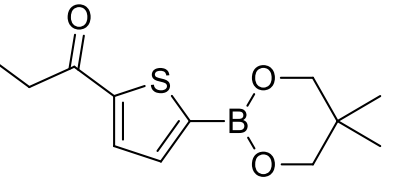

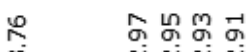

ำ ำ ำ

i ving

ij

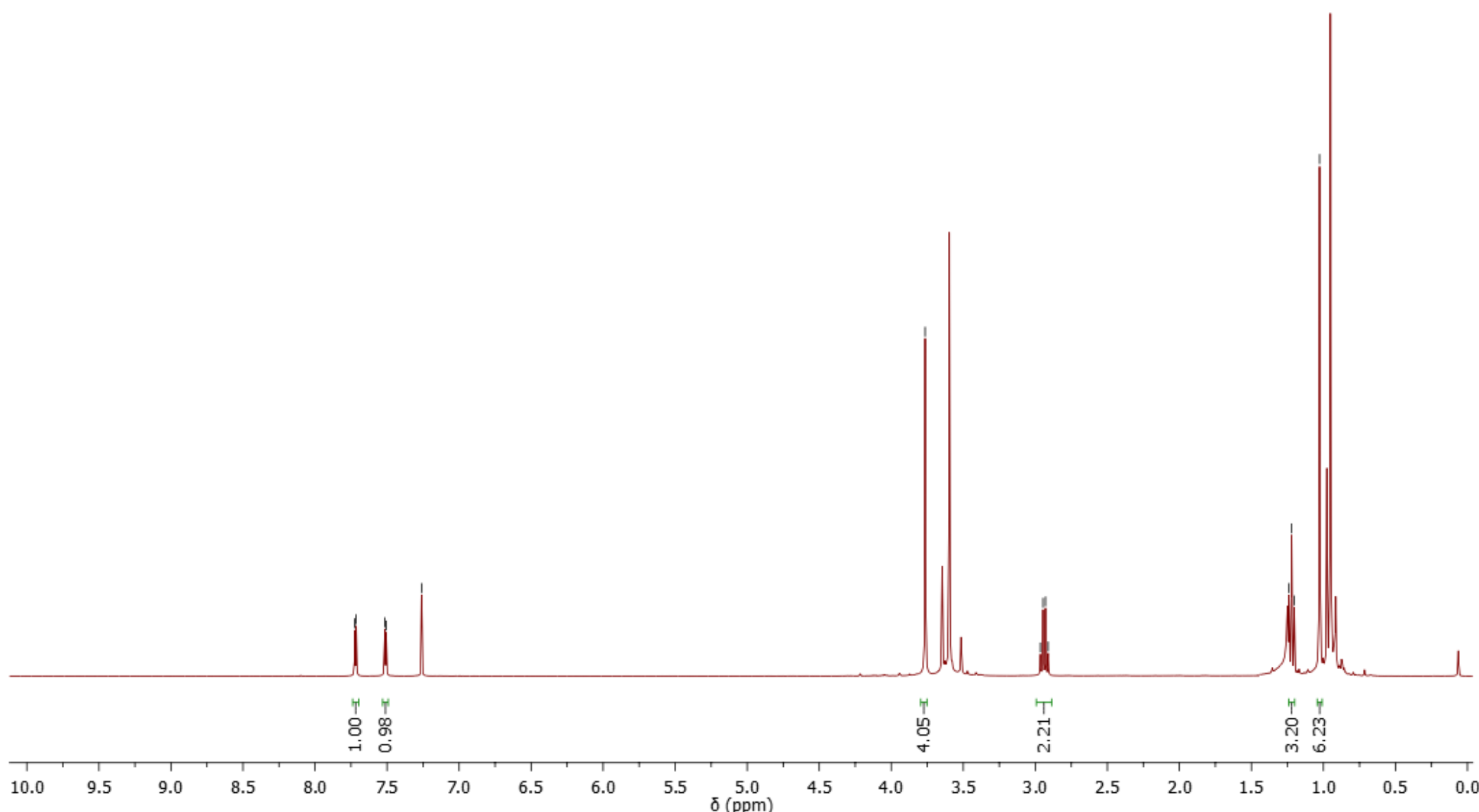


$\underline{\text { Thiophene boronate esters }}$

${ }^{13} \mathrm{C} 101 \mathrm{MHz}$

अं

ले ने

$\stackrel{\infty}{+}$

î

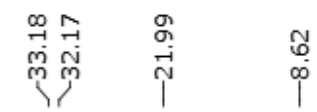

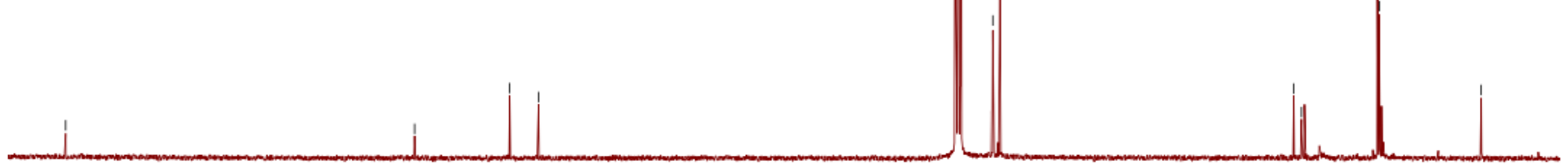


$\underline{\text { Thiophene boronate esters }}$

${ }^{1} \mathrm{H} 400 \mathrm{MHz}, \mathrm{CDCl}_{3}$
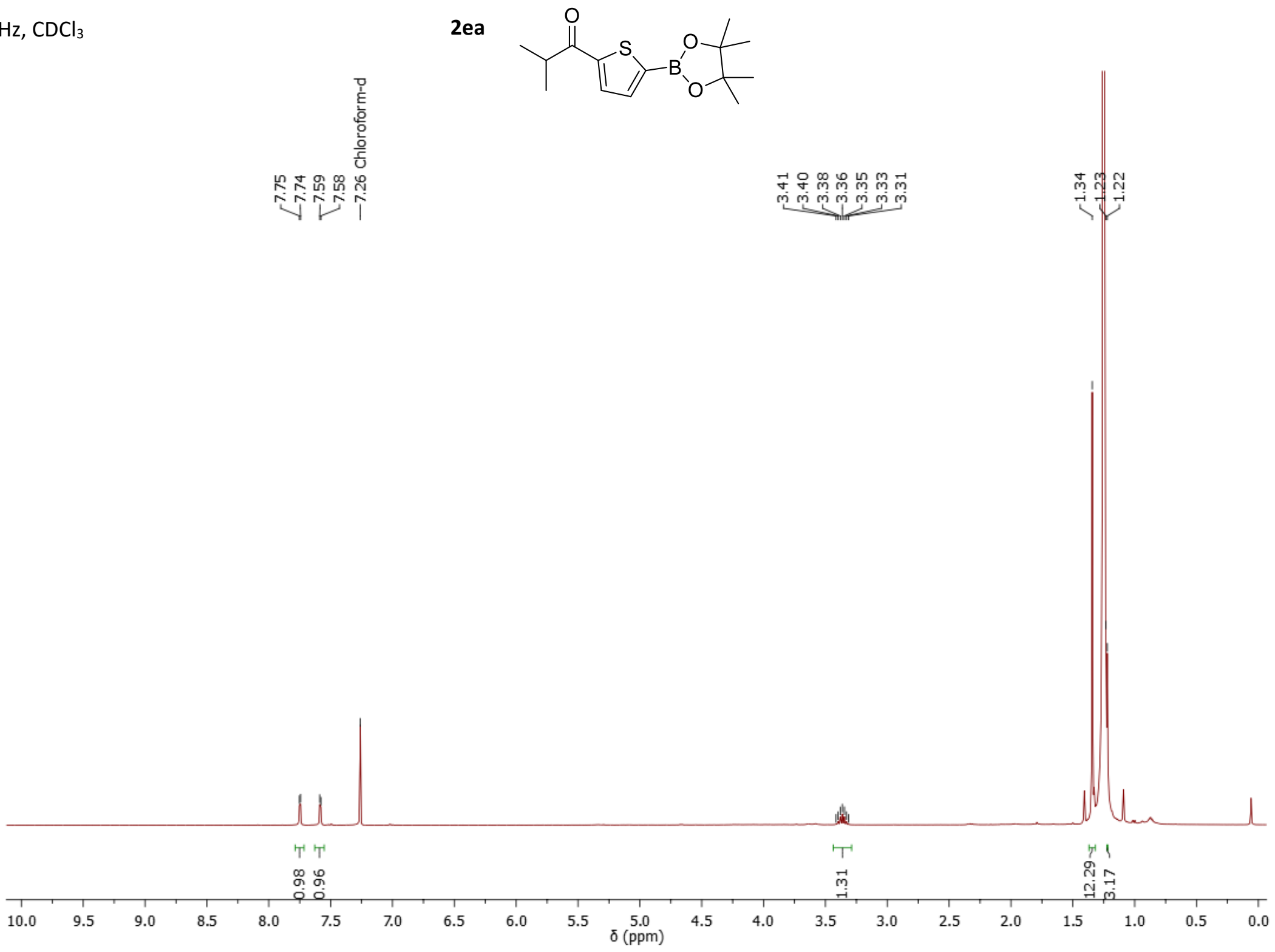
Thiophene boronate esters

${ }^{13} \mathrm{C} 101 \mathrm{MHz}$

商 2ea

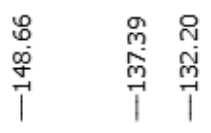
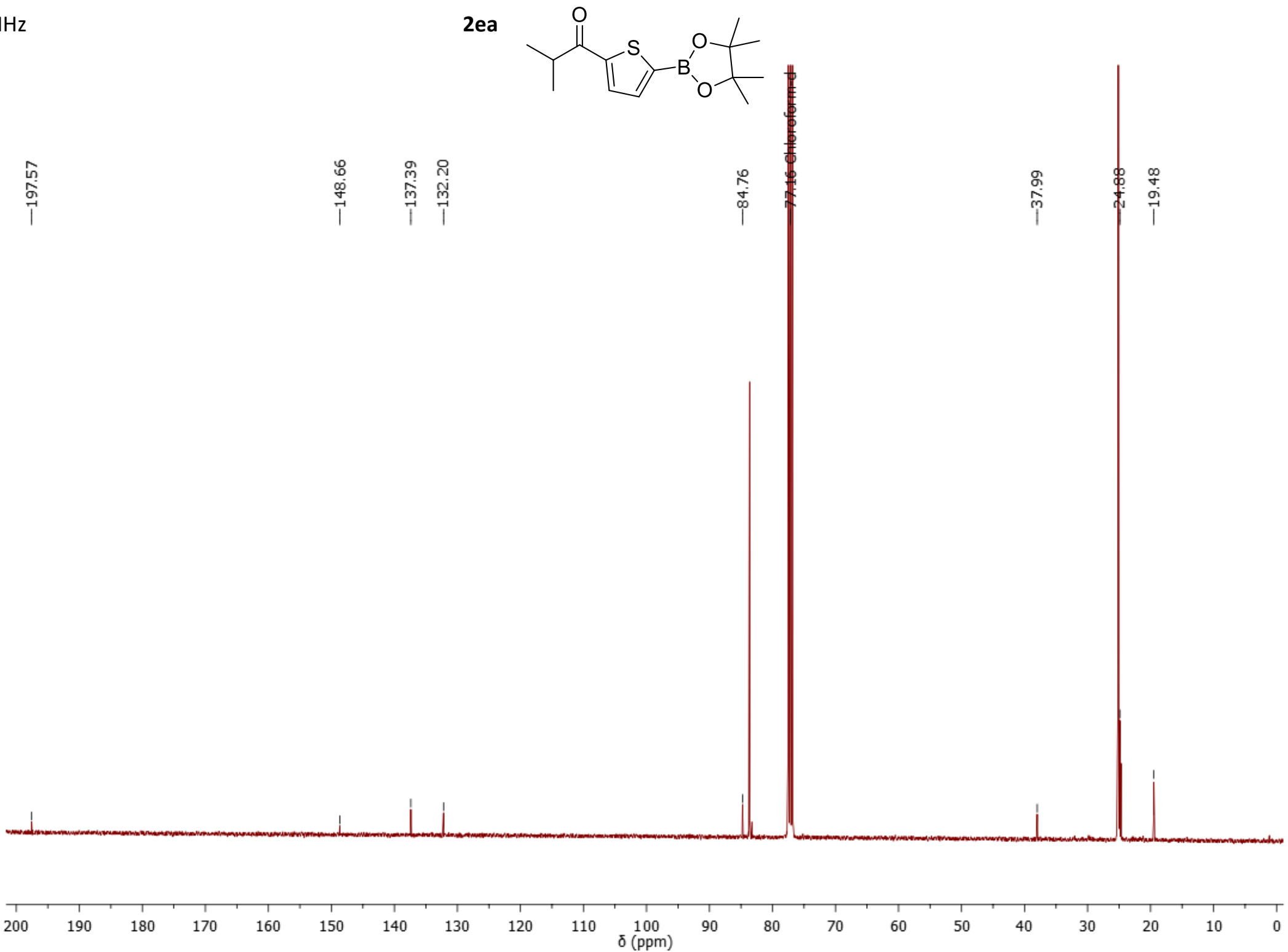

S86 
$\underline{\text { Thiophene boronate esters }}$

${ }^{1} \mathrm{H} 400 \mathrm{MHz}, \mathrm{CDCl}_{3}$

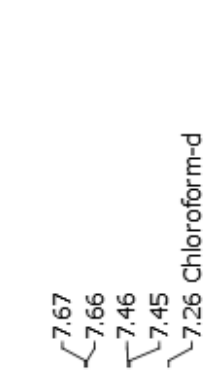

2eb

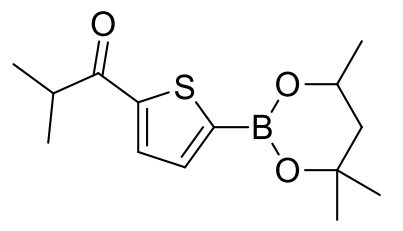

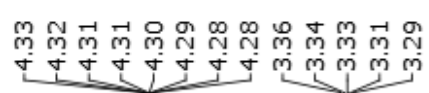

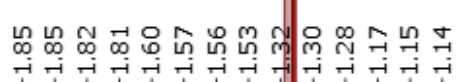

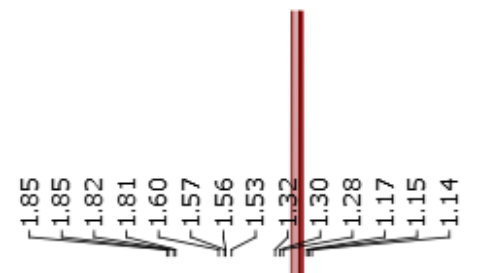


$\underline{\text { Thiophene boronate esters }}$

${ }^{13} \mathrm{C} 101 \mathrm{MHz}$
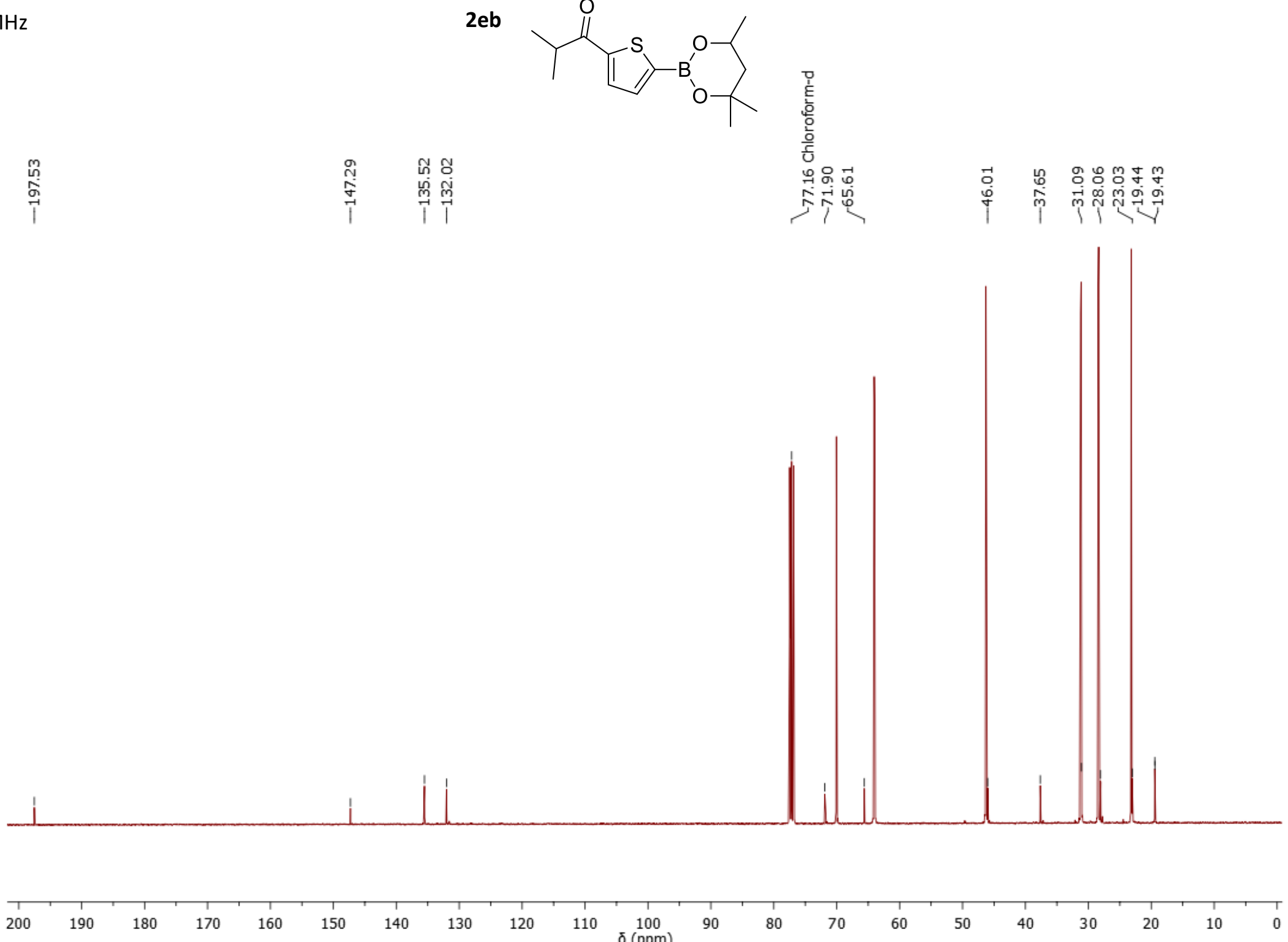
$\underline{\text { Thiophene boronate esters }}$

${ }^{1} \mathrm{H} 400 \mathrm{MHz}, \mathrm{CDCl}_{3}$

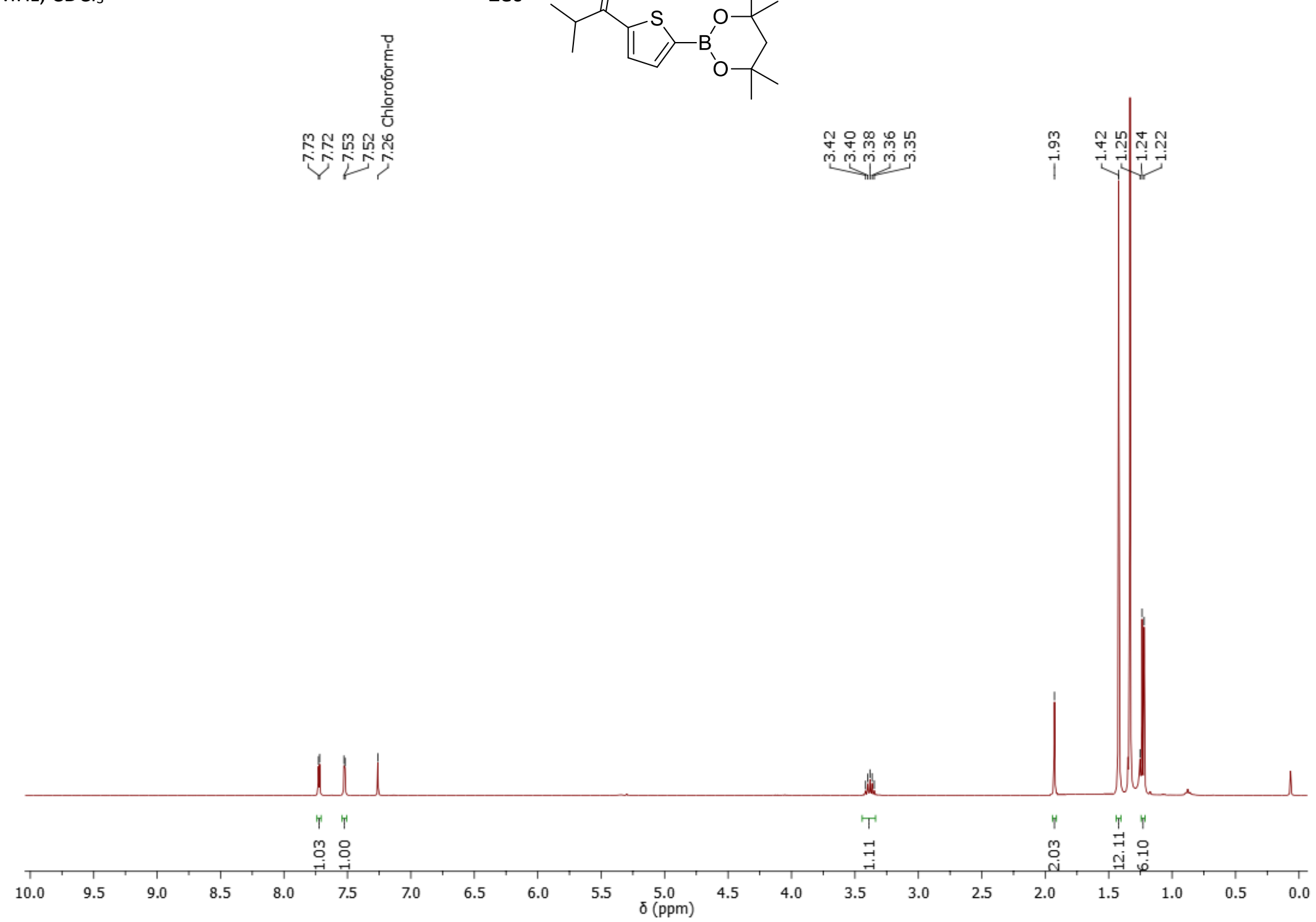


$\underline{\text { Thiophene boronate esters }}$

${ }^{13} \mathrm{C} 101 \mathrm{MHz}$

$\stackrel{\substack{\infty \\ \stackrel{\infty}{\sigma}}}{\stackrel{1}{+}}$

†

i

$2 e c$

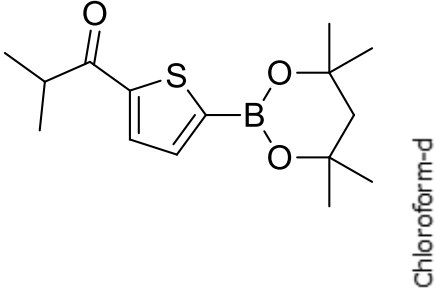

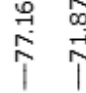

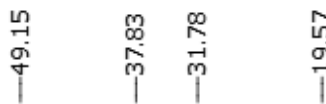


$\underline{\text { Thiophene boronate esters }}$

${ }^{1} \mathrm{H} 400 \mathrm{MHz}, \mathrm{CDCl}_{3}$

2ed

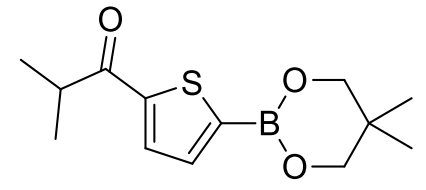

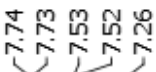

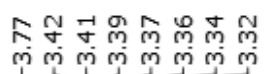

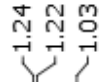

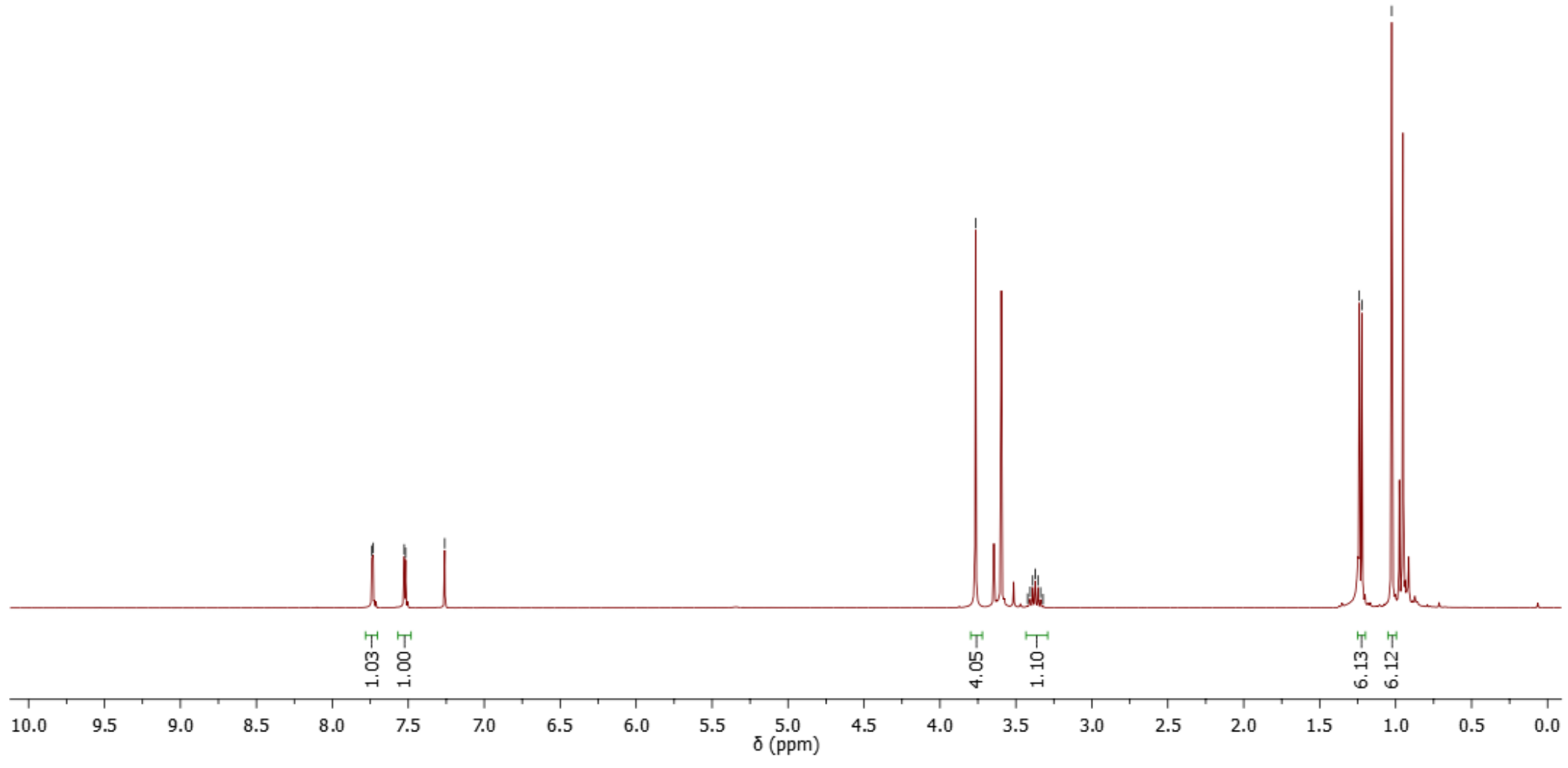


$\underline{\text { Thiophene boronate esters }}$

${ }^{13} \mathrm{C} 101 \mathrm{MHz}$

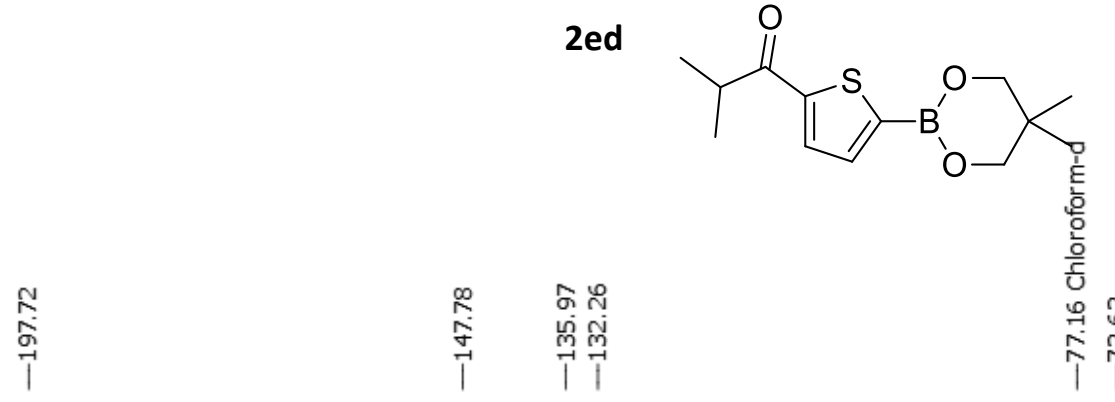

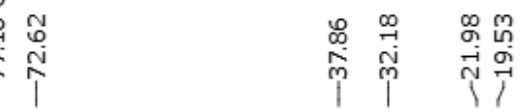

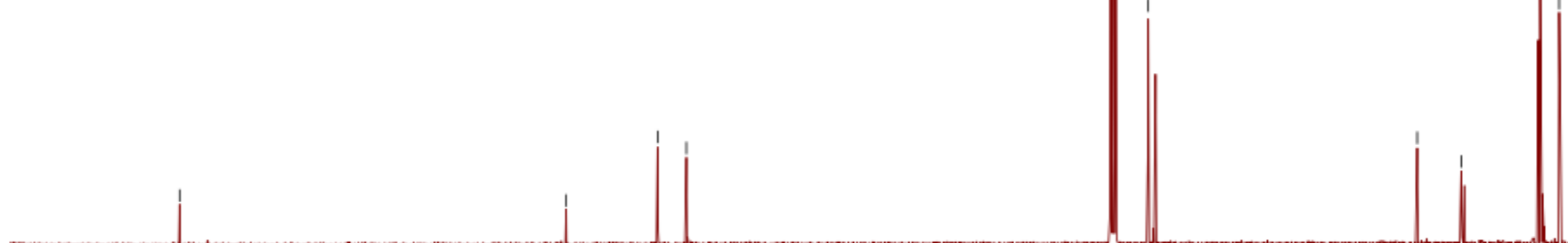

$\begin{array}{llllllllllll}210 & 200 & 190 & 180 & 170 & 160 & 150 & 140 & 130 & 120 & 110 & 100 \\ & & & & & & & & & & \end{array}$ 
$\underline{\text { Thiophene boronate esters }}$

${ }^{1} \mathrm{H} 400 \mathrm{MHz}, \mathrm{CDCl}_{3}$

$2 f a$

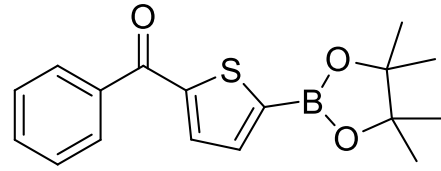

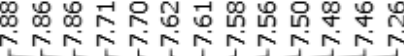

紊

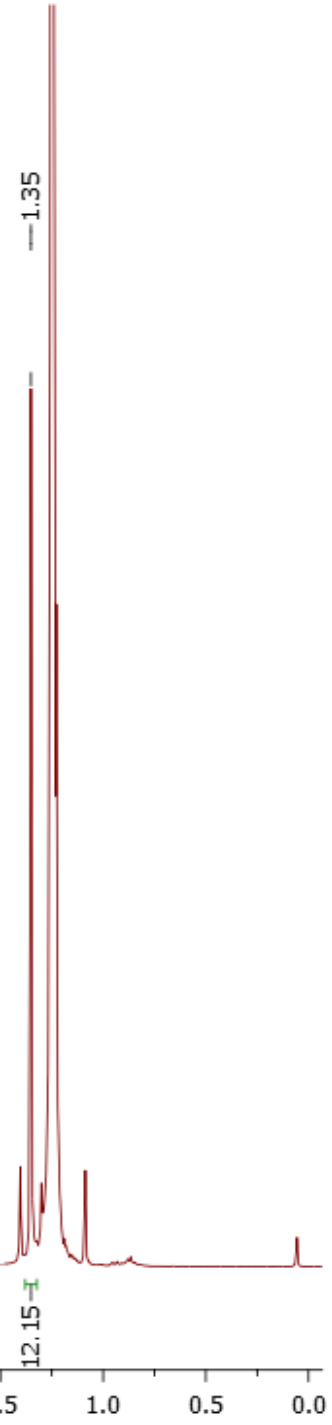


Thiophene boronate esters

${ }^{13} \mathrm{C} 101 \mathrm{MHz}$

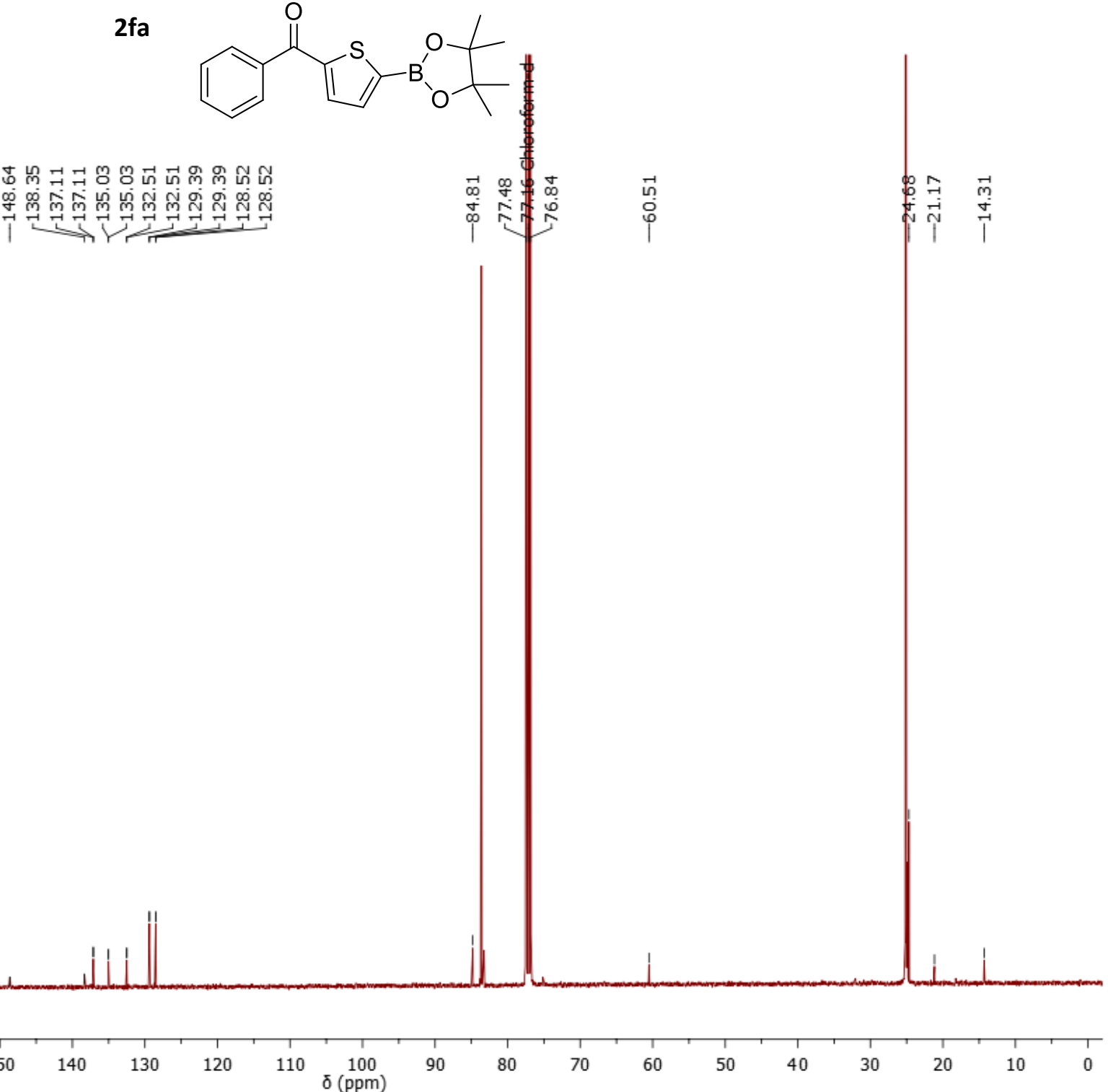


$\underline{\text { Thiophene boronate esters }}$

${ }^{1} \mathrm{H} 400 \mathrm{MHz}, \mathrm{CDCl}_{3}$

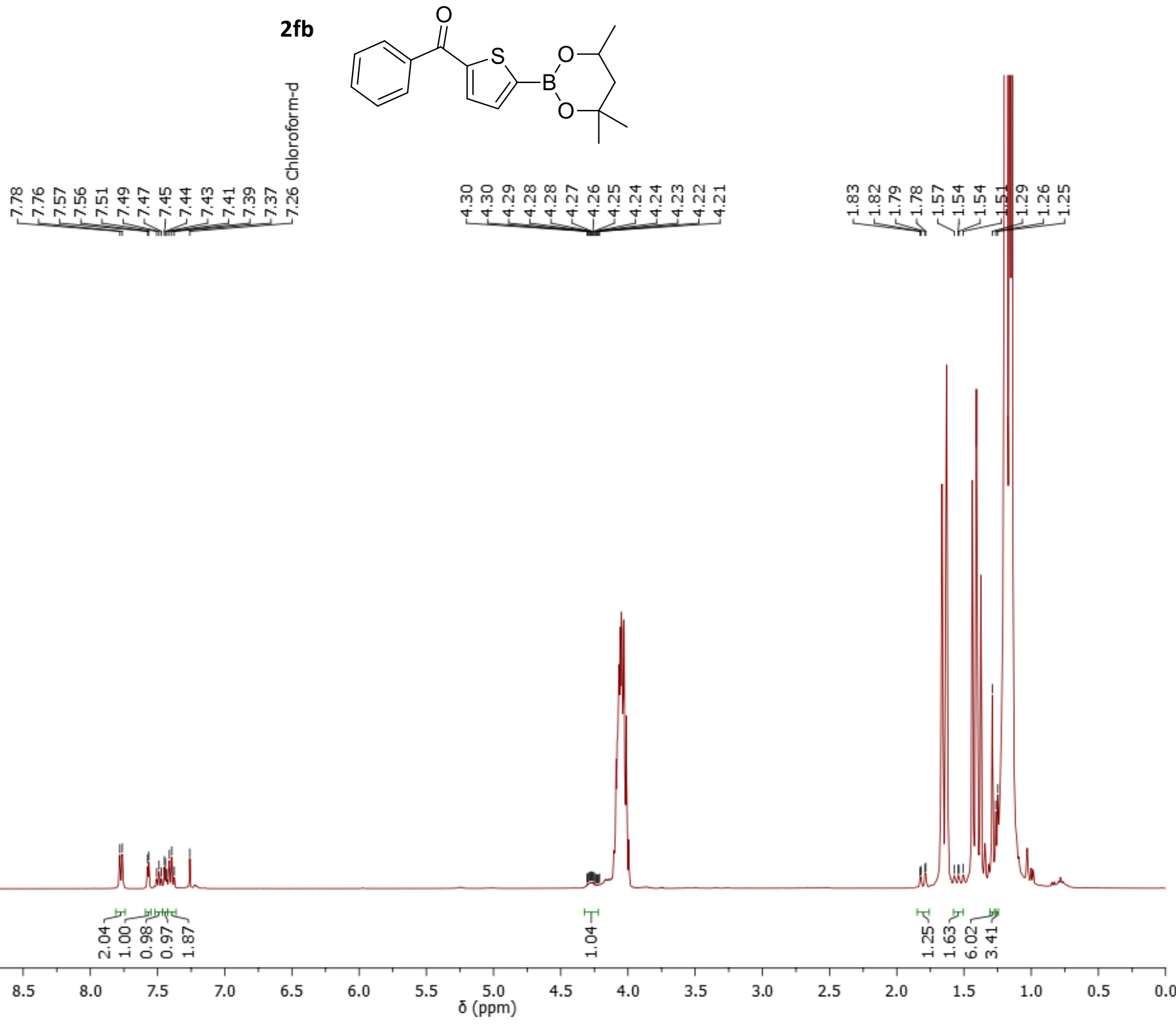


$\underline{\text { Thiophene boronate esters }}$

${ }^{13} \mathrm{C} 101 \mathrm{MHz}$

$2 \mathrm{fb}$

\begin{tabular}{l}
0 \\
$\infty$ \\
$\infty$ \\
\hline
\end{tabular}

․ ल굥ㅇํㅇ

察

눈

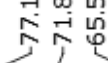

ம0 के

अ गัNญ

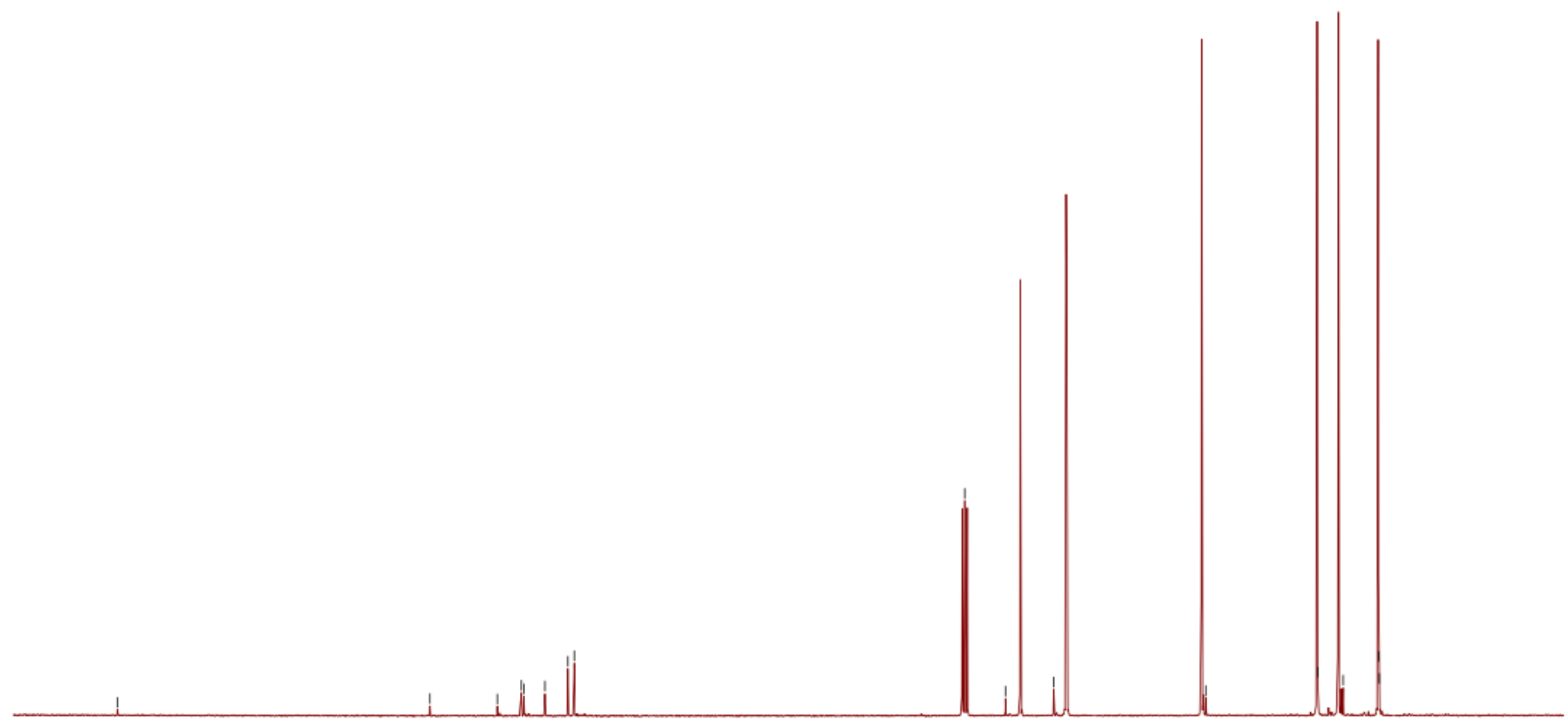


$\underline{\text { Thiophene boronate esters }}$

${ }^{1} \mathrm{H} 400 \mathrm{MHz}, \mathrm{CDCl}_{3}$

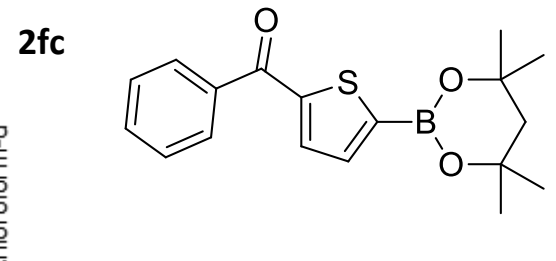

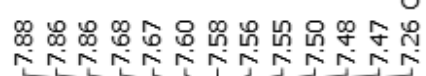

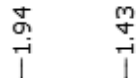

Uit
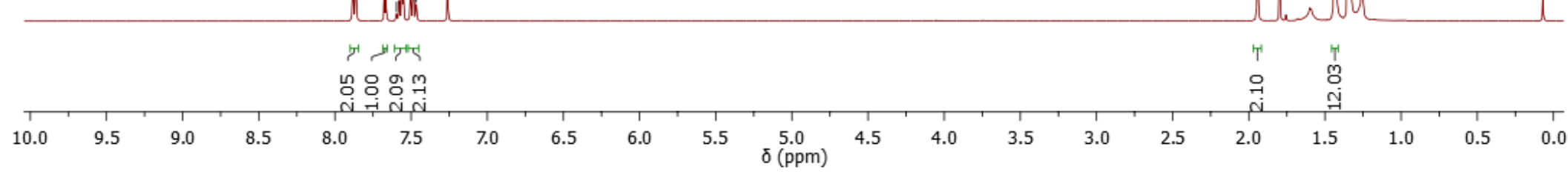
$\underline{\text { Thiophene boronate esters }}$

${ }^{13} \mathrm{C} 101 \mathrm{MHz}$
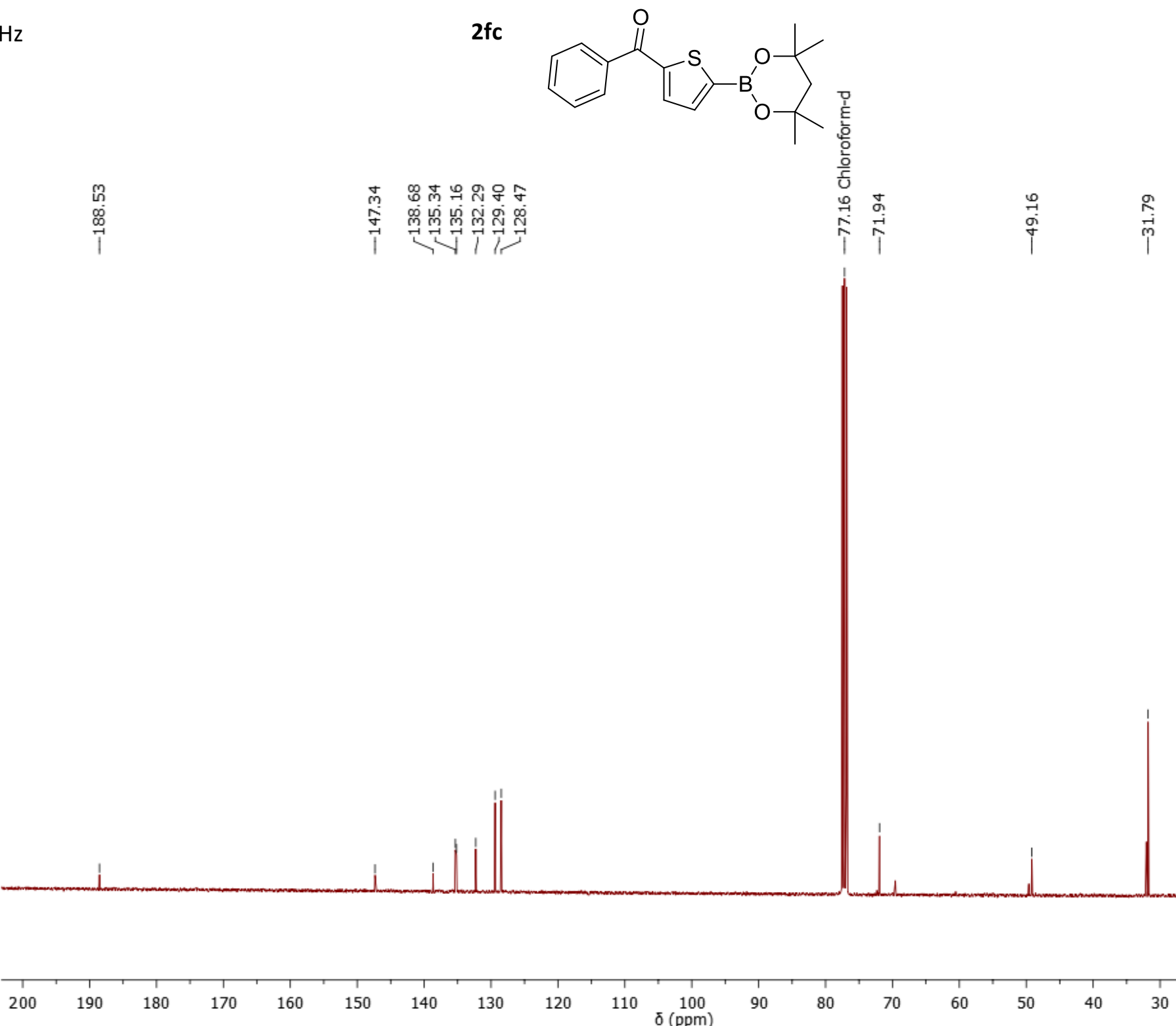
$\underline{\text { Thiophene boronate esters }}$

${ }^{1} \mathrm{H} 400 \mathrm{MHz}, \mathrm{CDCl}_{3}$

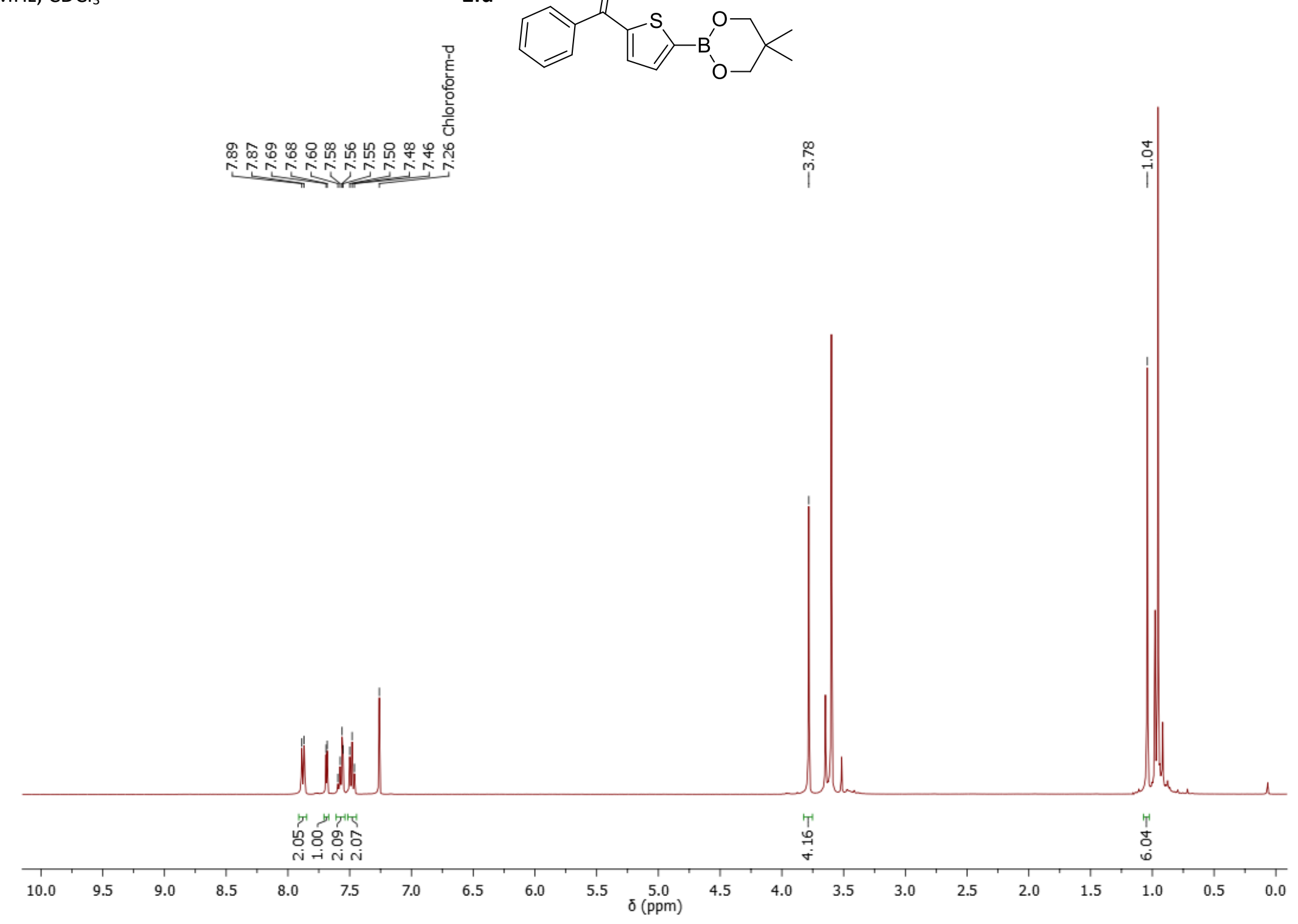


$\underline{\text { Thiophene boronate esters }}$

${ }^{13} \mathrm{C} 101 \mathrm{MHz}$

$2 \mathrm{fd}$

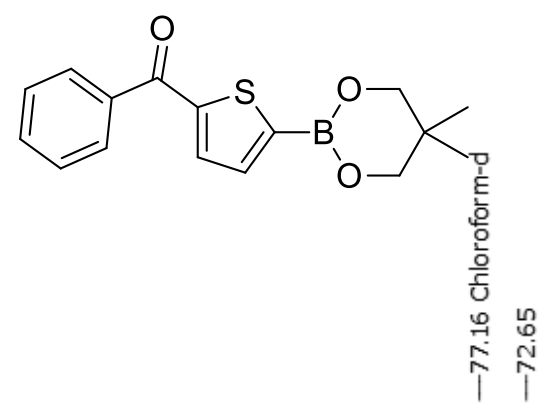

$\begin{array}{ll}\stackrel{9}{N} & 8 \\ i & N \\ i & N\end{array}$

$200 \quad 190$

$70 \quad 160$

150

$140 \quad 130$

120

$\begin{array}{cc}110 & 100 \\ \delta(\mathrm{ppm}) & \end{array}$

90

$\begin{array}{ll}1 & 1 \\ 80 & 70\end{array}$

1
$60 \quad 50$

40

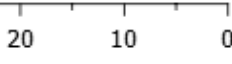




\section{Furan boronate esters}

${ }^{1} \mathrm{H} 400 \mathrm{MHz}, \mathrm{CDCl}_{3}$

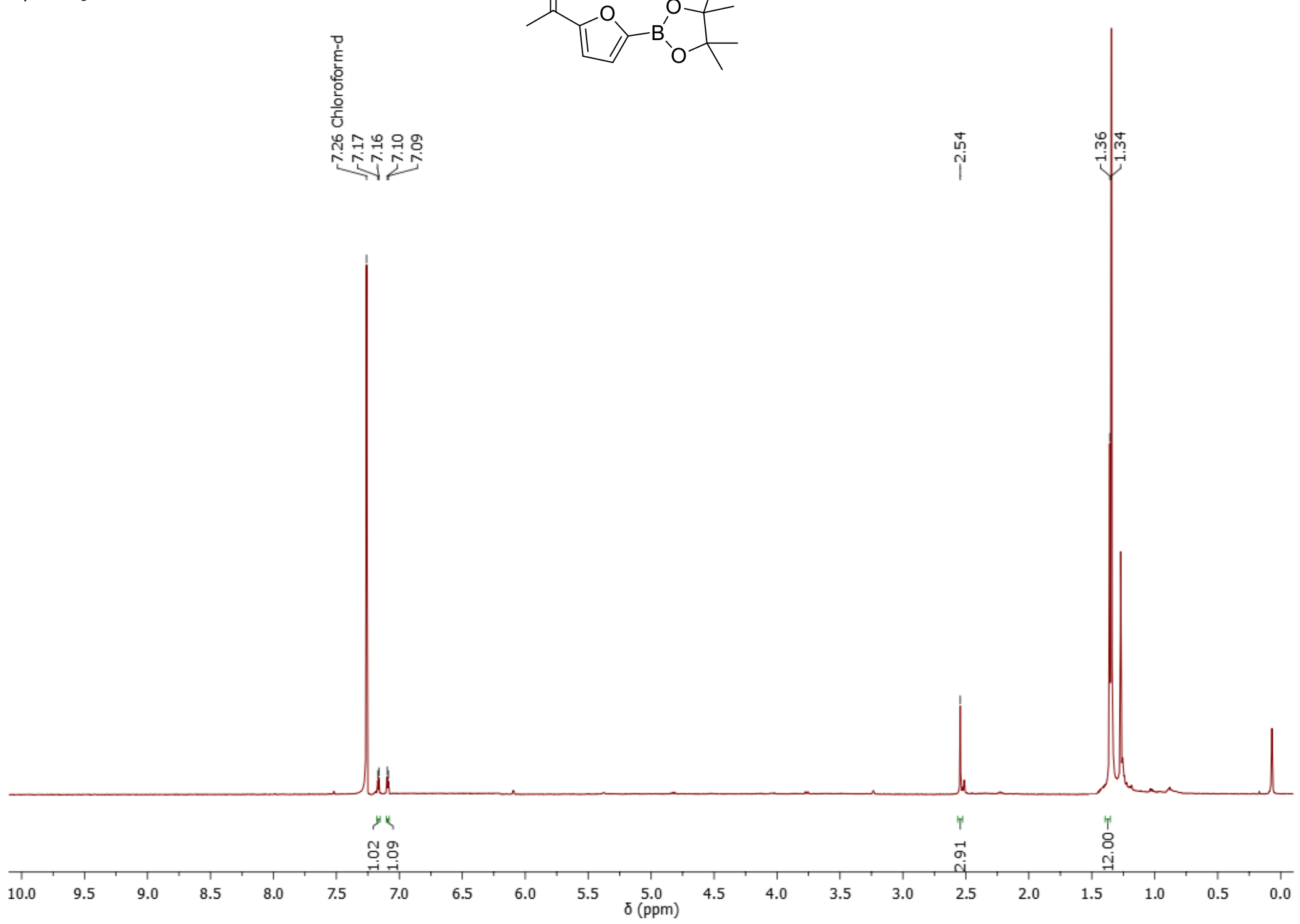




$$
1
$$


Furan boronate esters

${ }^{1} \mathrm{H} 400 \mathrm{MHz}, \mathrm{CDCl}_{3}$

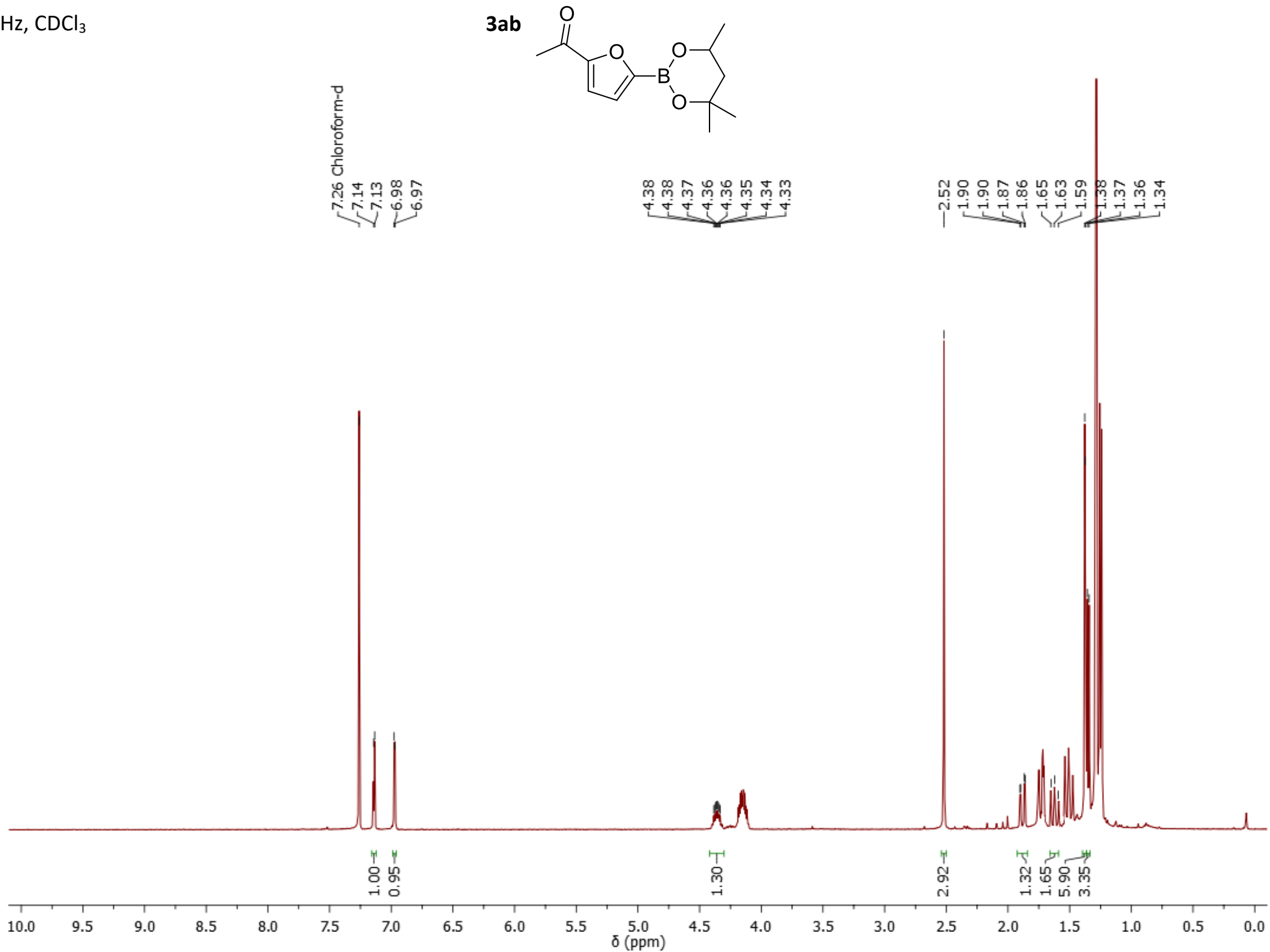

$3 a b$

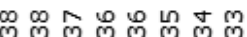

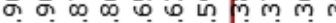


Furan boronate esters

${ }^{13} \mathrm{C} 101 \mathrm{MHz}$

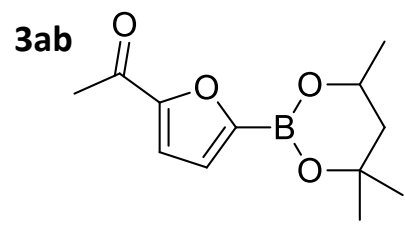

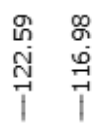

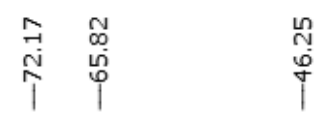

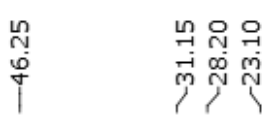

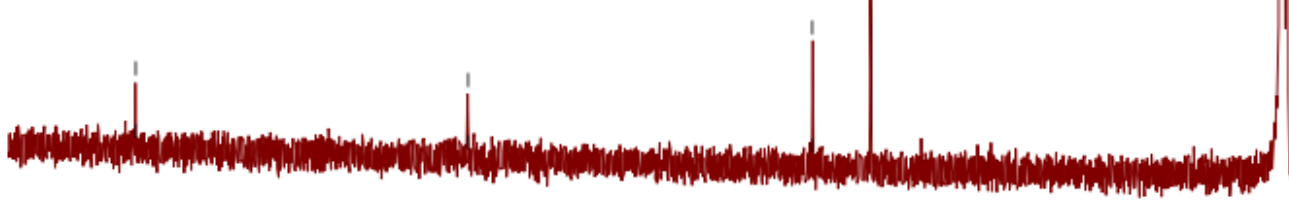
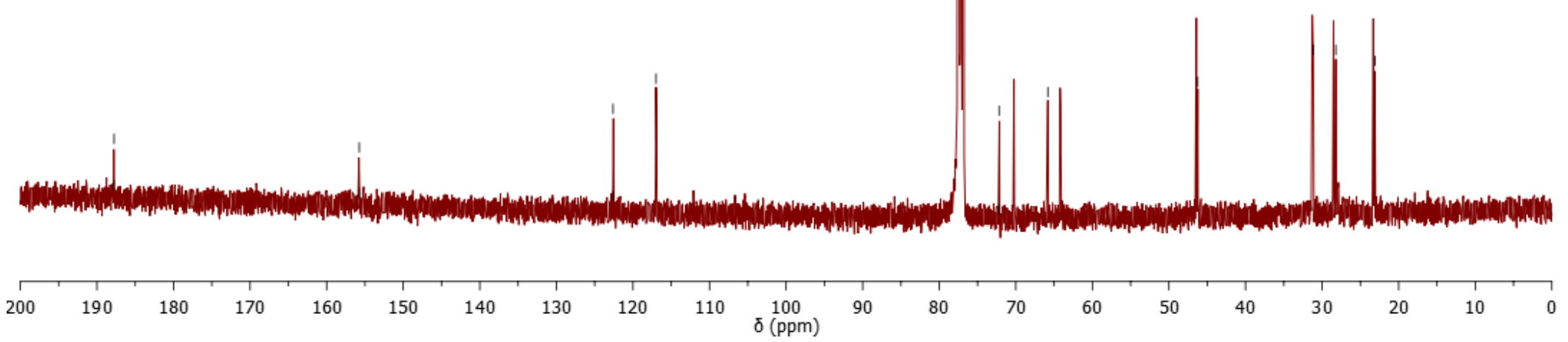

S104 


\section{Furan boronate esters}

${ }^{1} \mathrm{H} 400 \mathrm{MHz}, \mathrm{CDCl}_{3}$

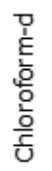

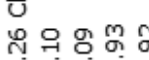

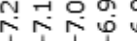

$3 a c$

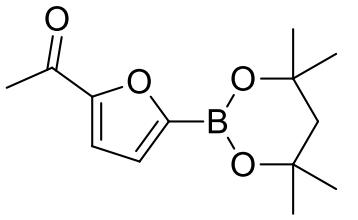

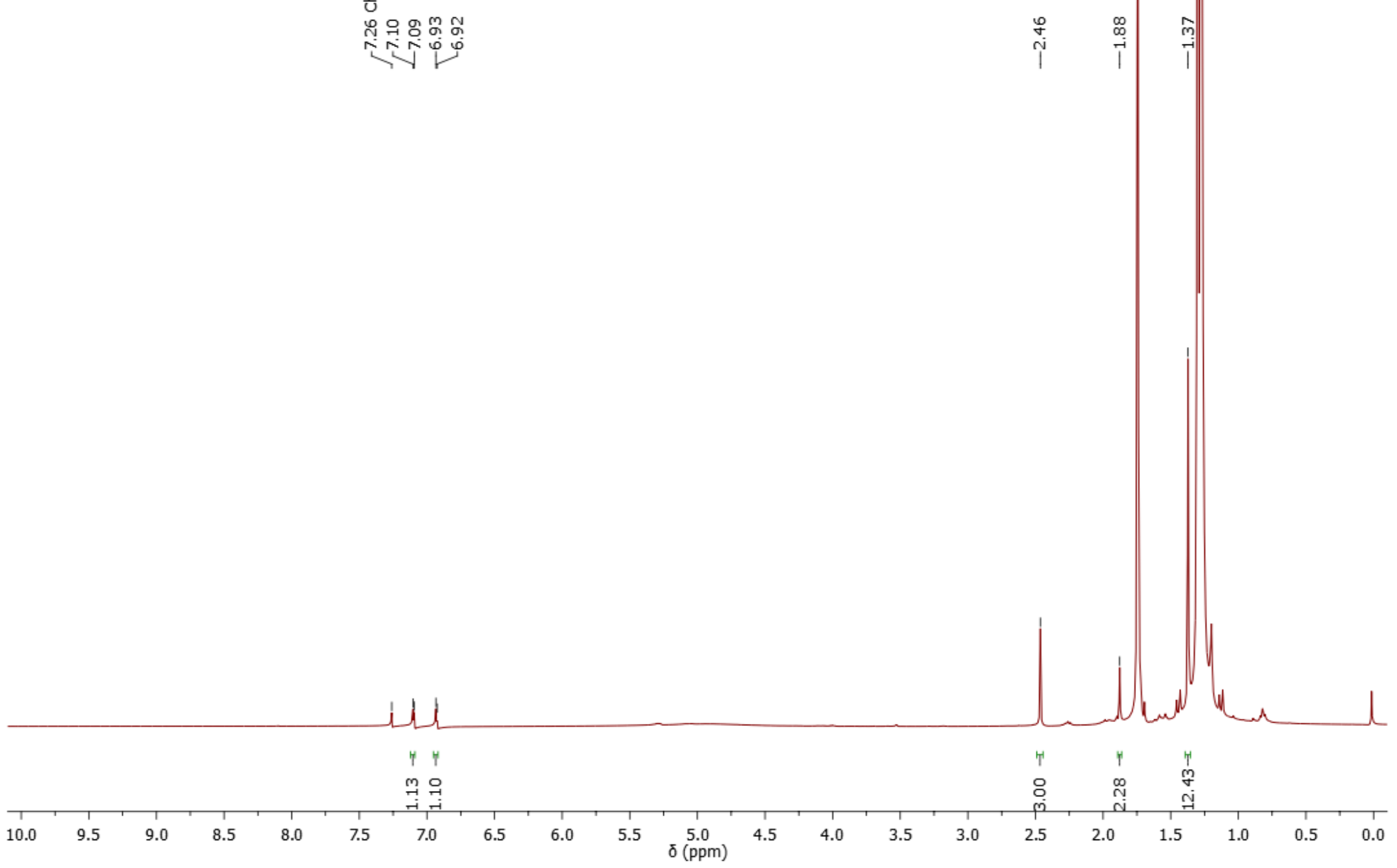


Furan boronate esters

${ }^{13} \mathrm{C} 101 \mathrm{MHz}$
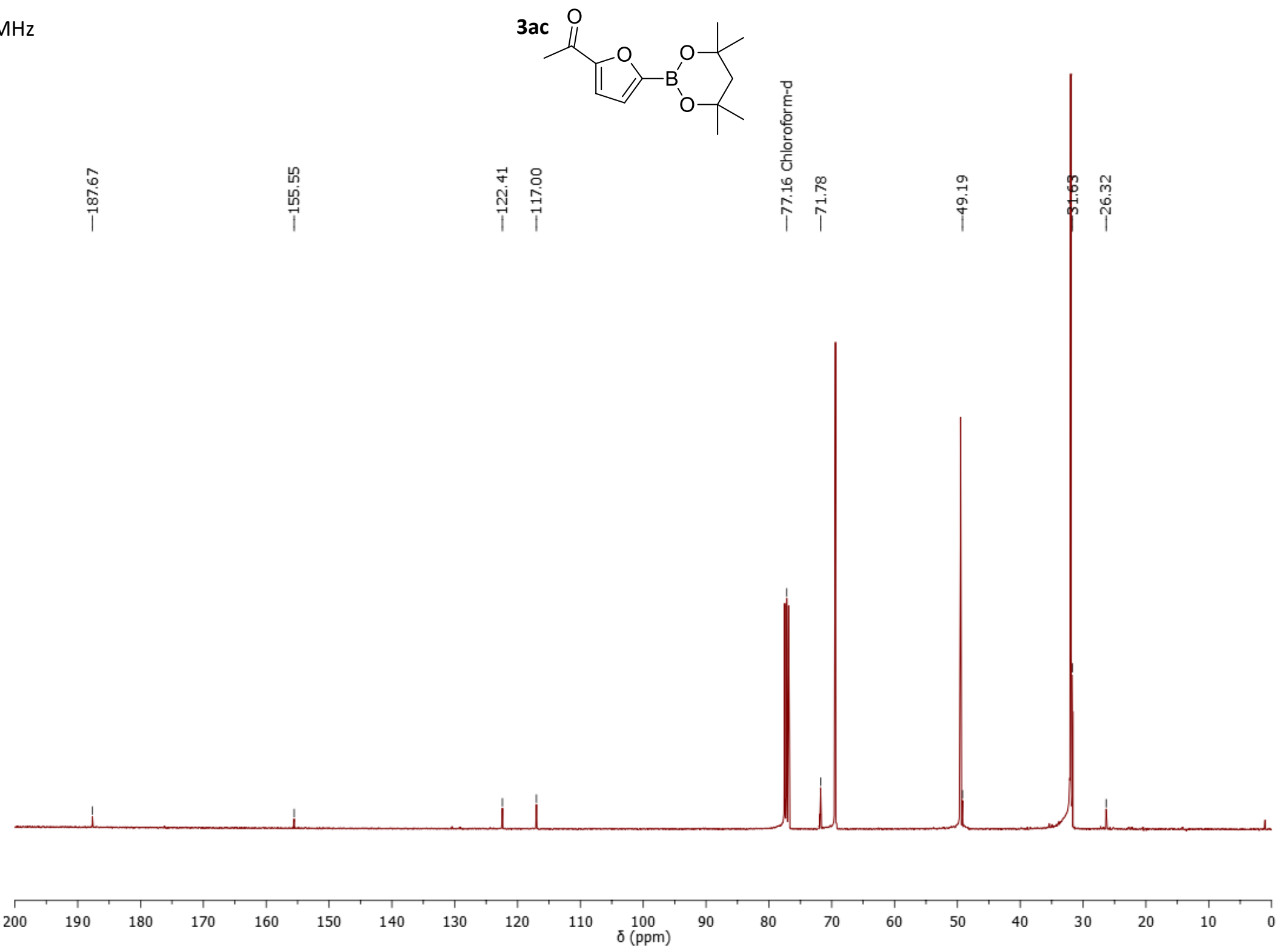

S106 


\section{Furan boronate esters}

${ }^{1} \mathrm{H} 400 \mathrm{MHz}, \mathrm{CDCl}_{3}$

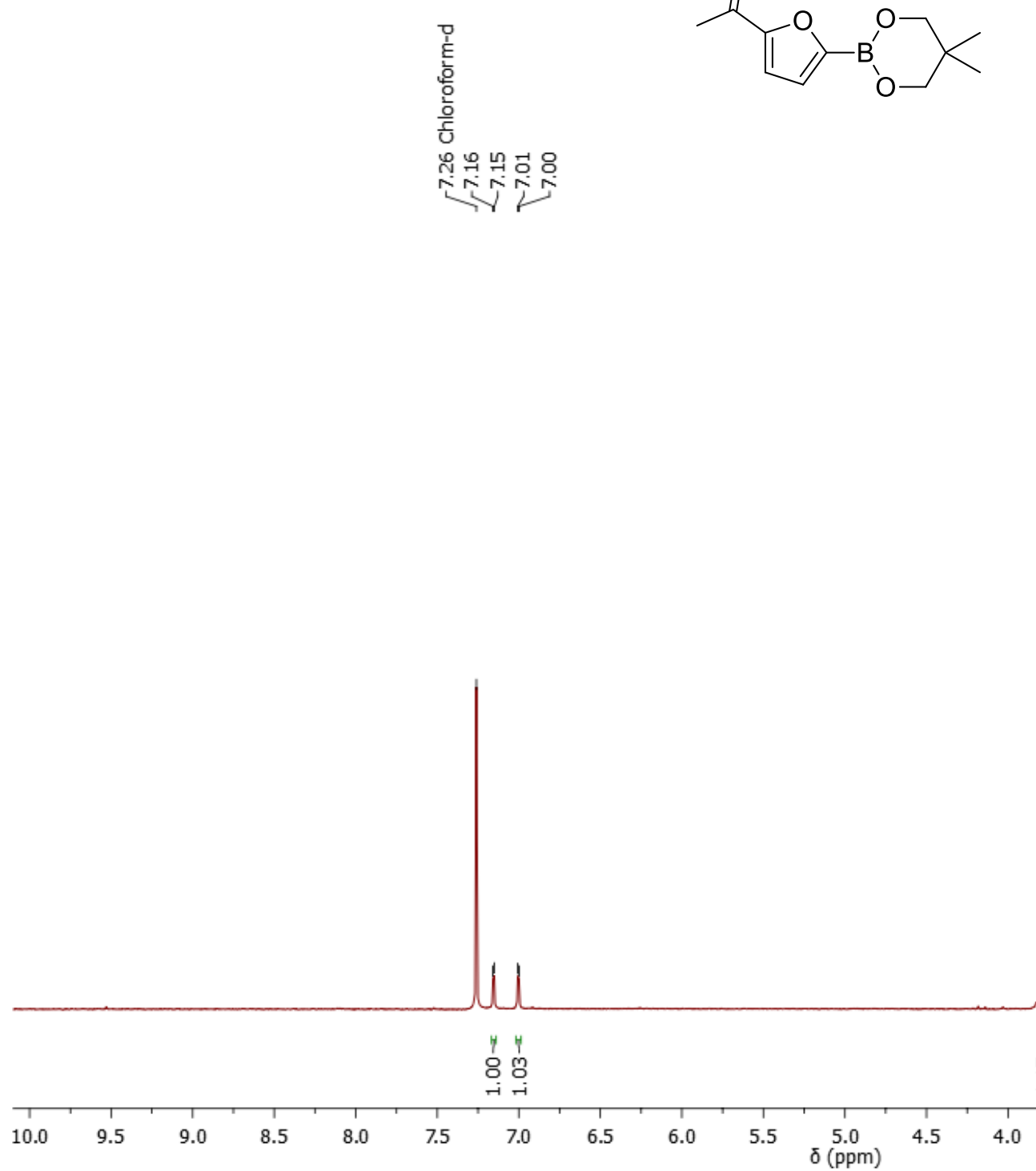

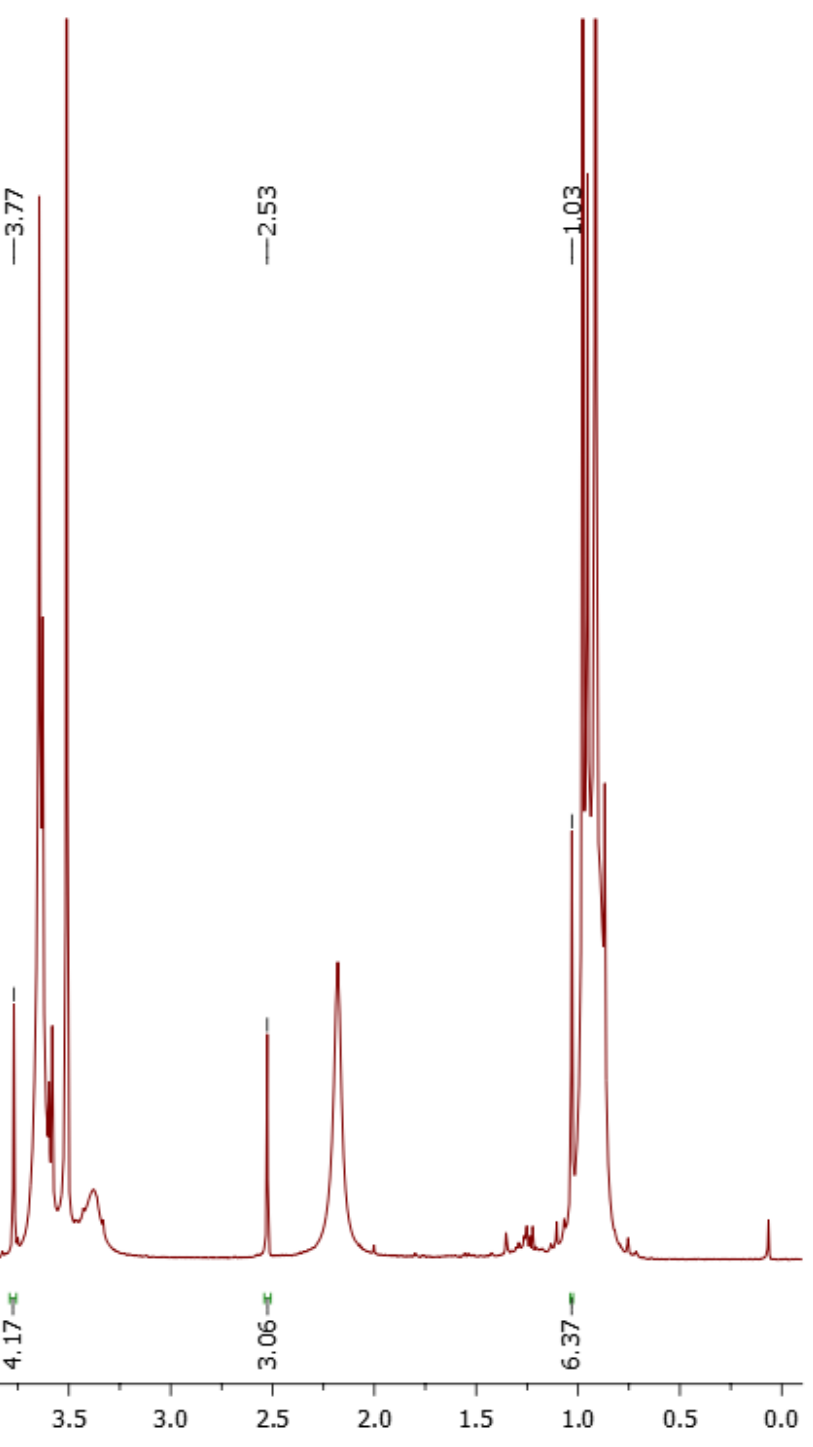


Furan boronate esters

${ }^{13} \mathrm{C} 101 \mathrm{MHz}$

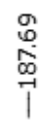

|

3ad

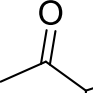

H

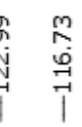

군

M

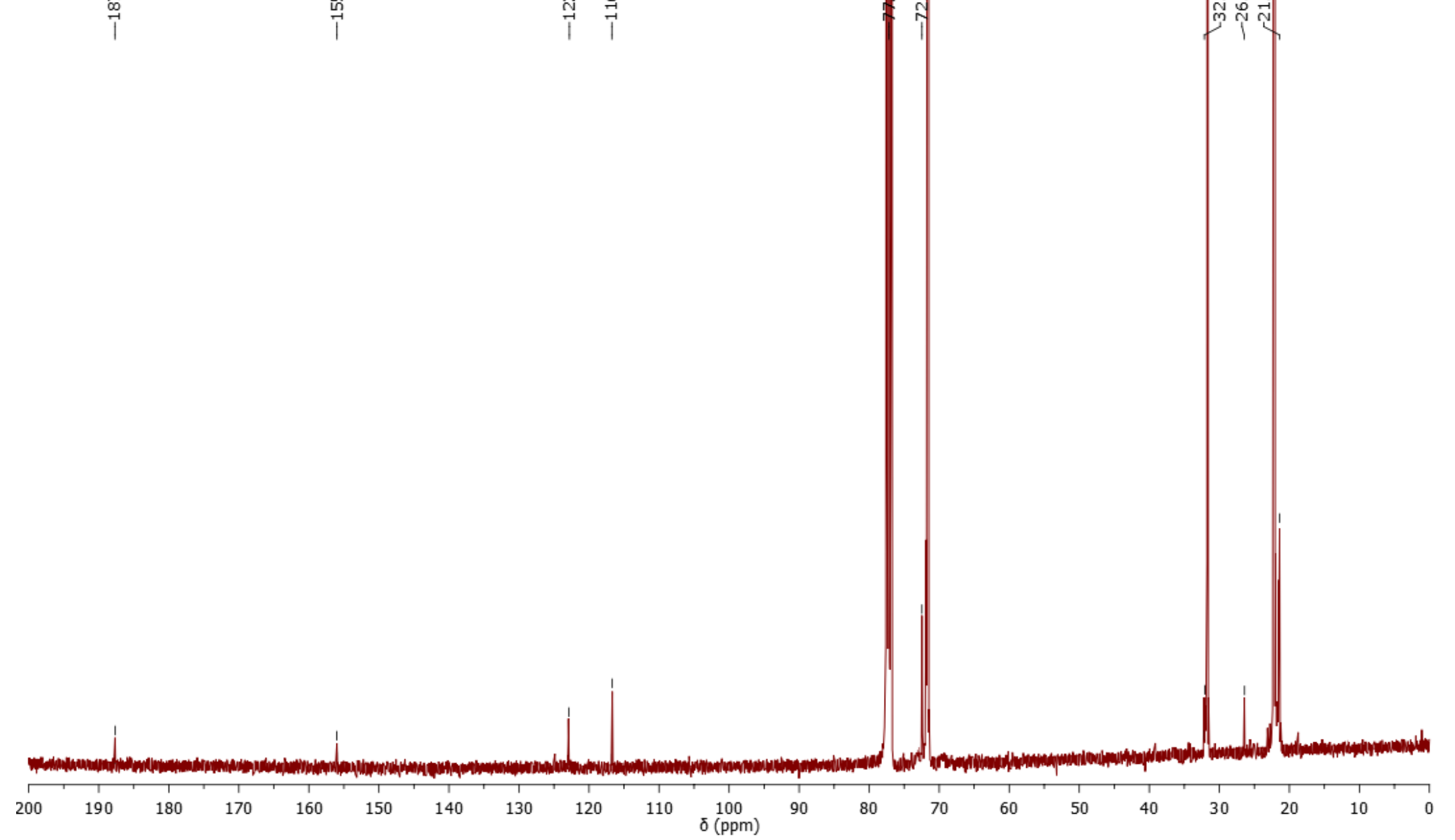


Furan boronate esters

${ }^{1} \mathrm{H} 400 \mathrm{MHz}, \mathrm{CDCl}_{3}$

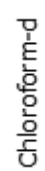

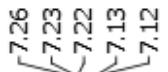

is
$3 b a$

II

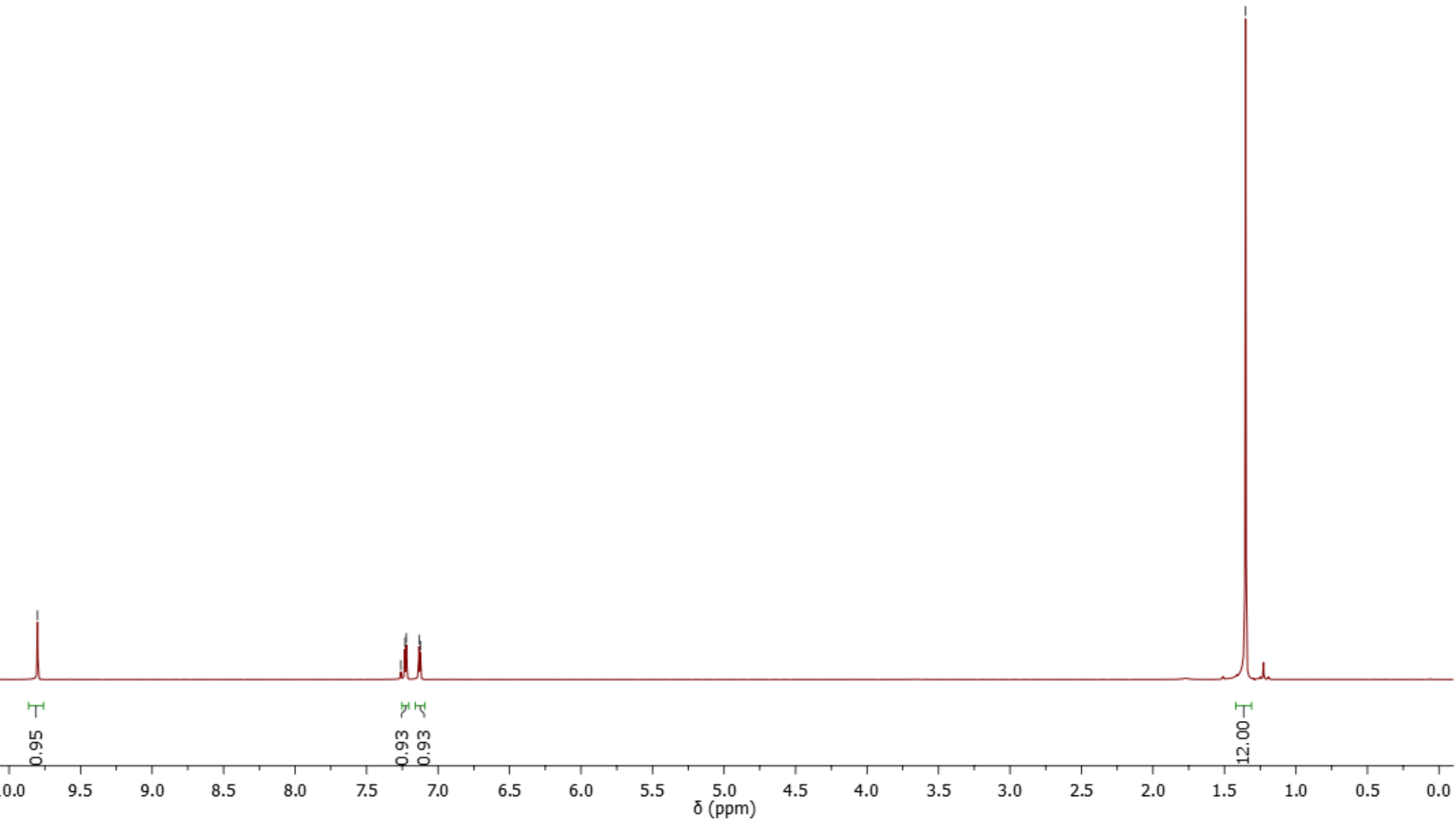


Furan boronate esters

${ }^{13} \mathrm{C} 101 \mathrm{MHz}, \mathrm{CDCl}_{3}$

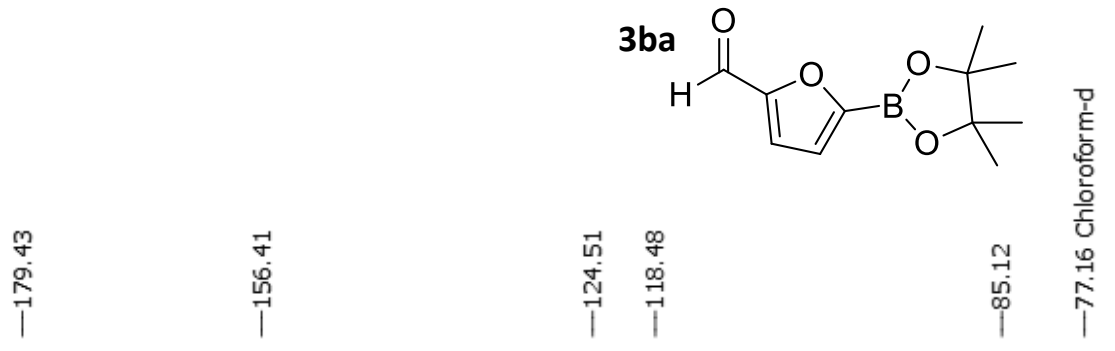

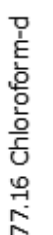

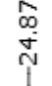

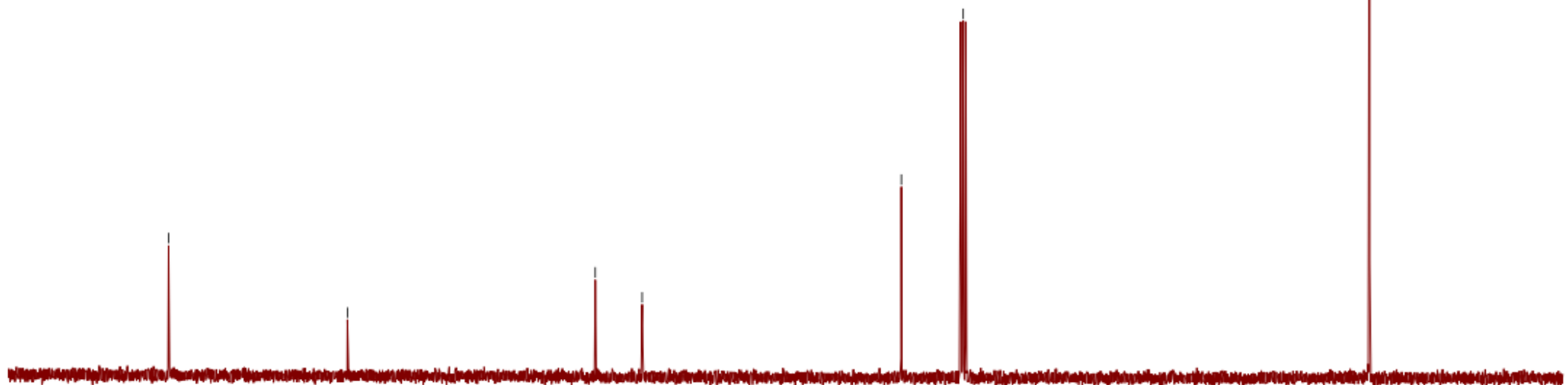

200

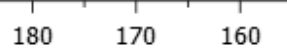

140

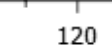

$110 \quad \begin{aligned} & 100 \\ & \delta(\mathrm{ppm})\end{aligned}$
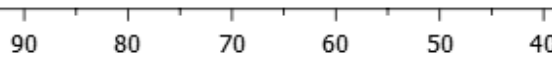

30 
Furan boronate esters

${ }^{1} \mathrm{H} 400 \mathrm{MHz}, \mathrm{CDCl}_{3}$

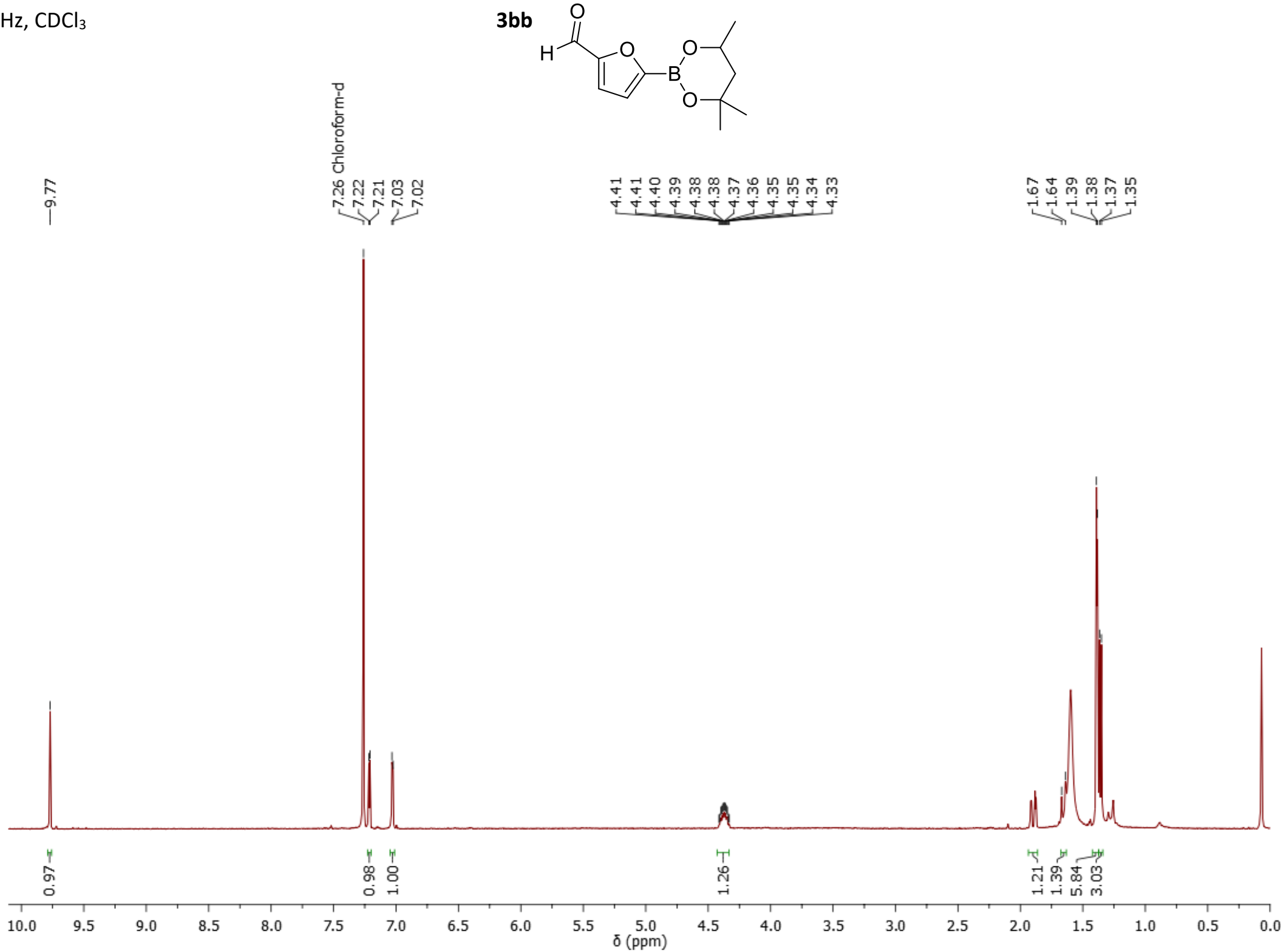

$3 b b$

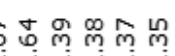

垈记 
Furan boronate esters

${ }^{13} \mathrm{C} 101 \mathrm{MHz}$

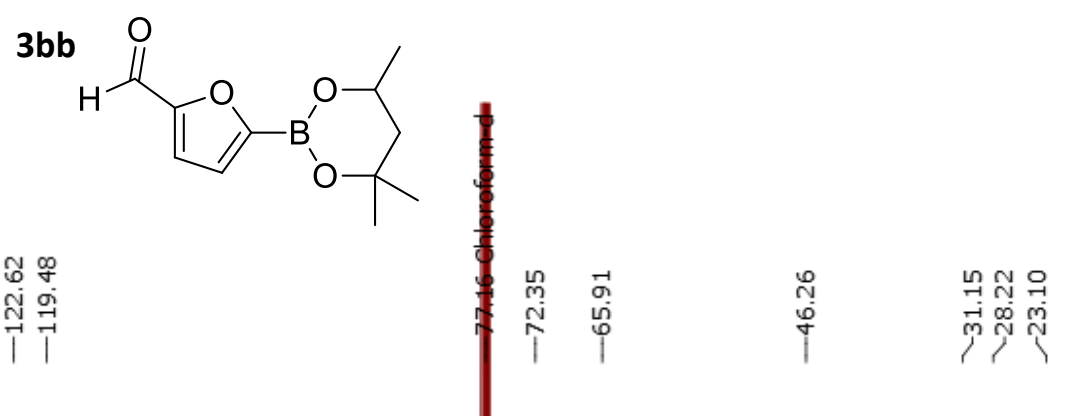

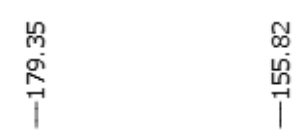

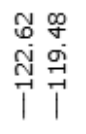

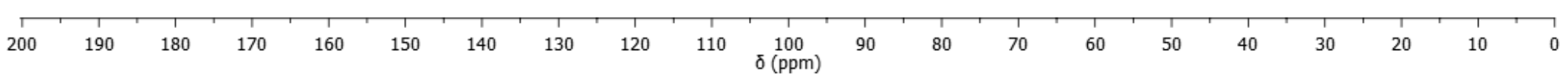

S112 
Furan boronate esters

${ }^{1} \mathrm{H} 400 \mathrm{MHz}, \mathrm{CDCl}_{3}$

$\underset{i}{\stackrel{R}{i}}$

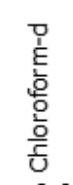

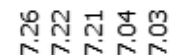

$3 b c$

$$
\text { ill }
$$

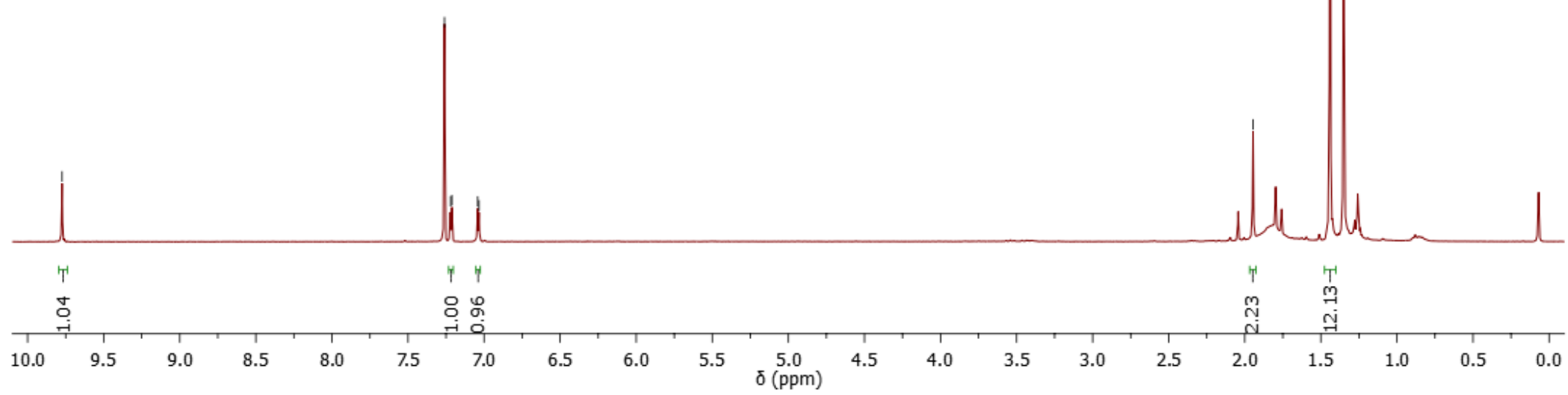


Furan boronate esters

${ }^{13} \mathrm{C} 101 \mathrm{MHz}$

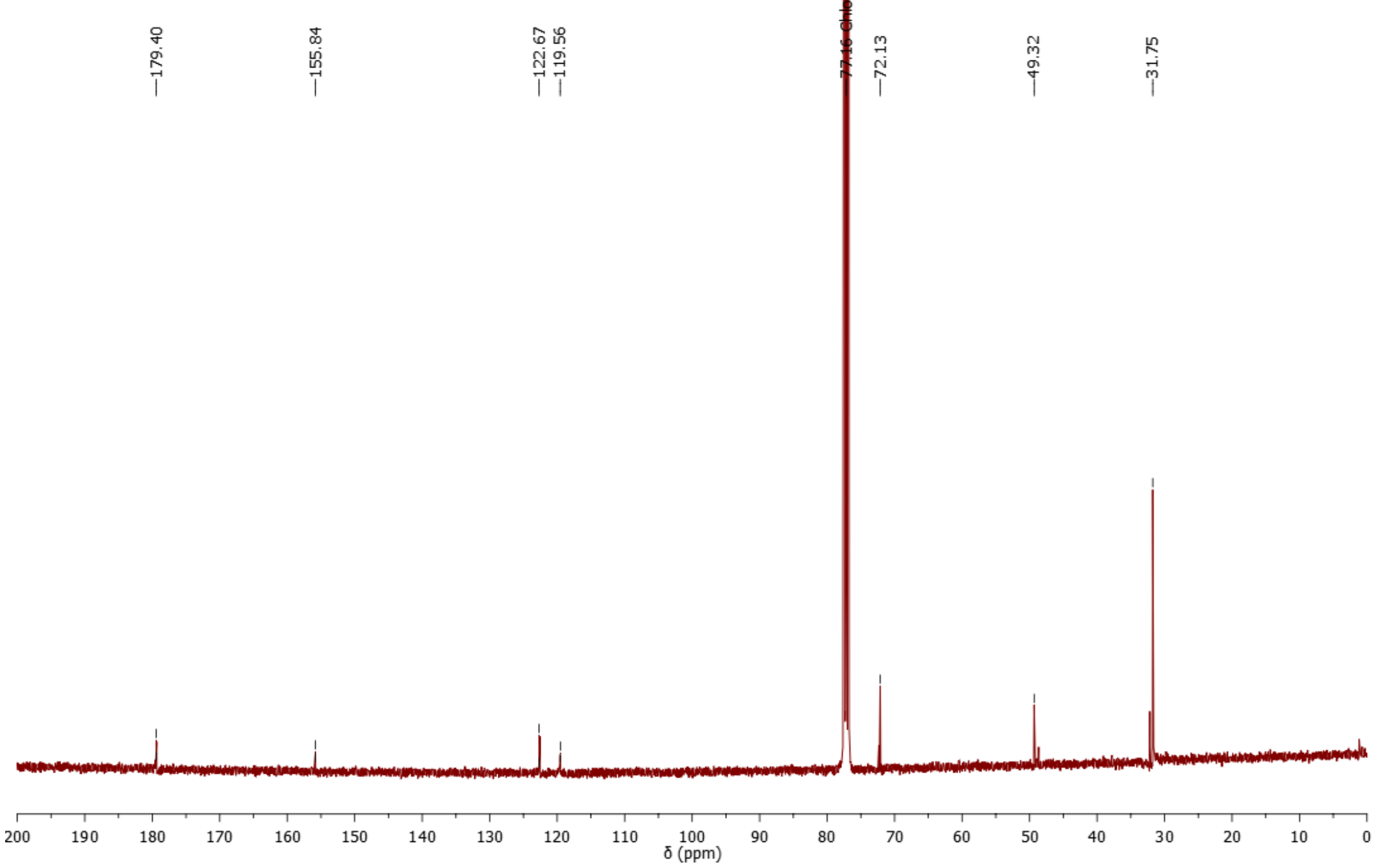

S114 
Furan boronate esters

${ }^{1} \mathrm{H} 400 \mathrm{MHz}, \mathrm{CDCl}_{3}$

3bd

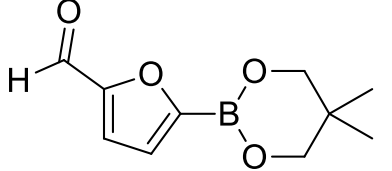

$\stackrel{\infty}{i}$

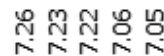

$\stackrel{\infty}{\stackrel{\infty}{p}}$

$\stackrel{\text { m }}{\stackrel{\text { i }}{i}}$

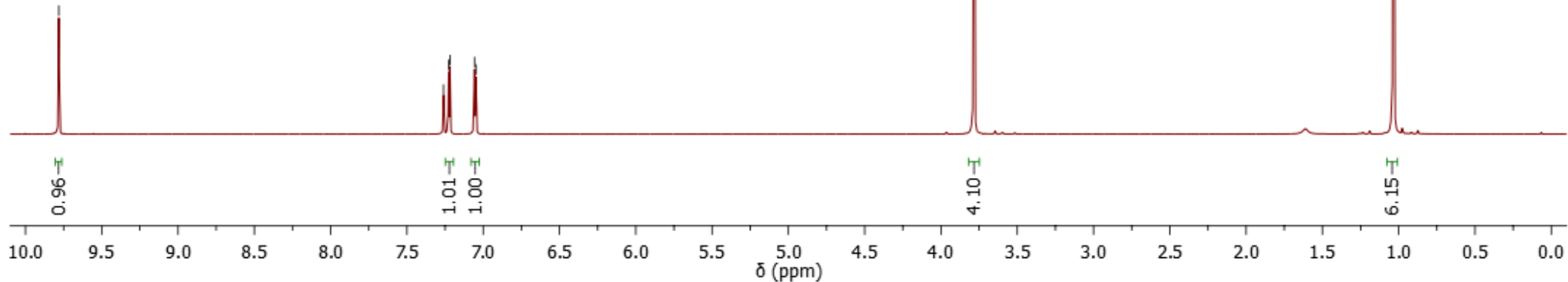


Furan boronate esters

${ }^{13} \mathrm{C} 101 \mathrm{MHz}$

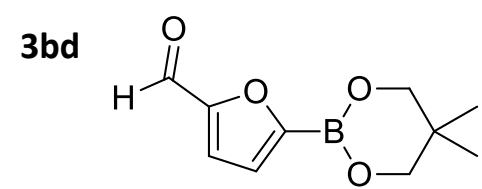

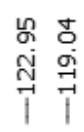

$\stackrel{\circ}{\stackrel{0}{i}}$
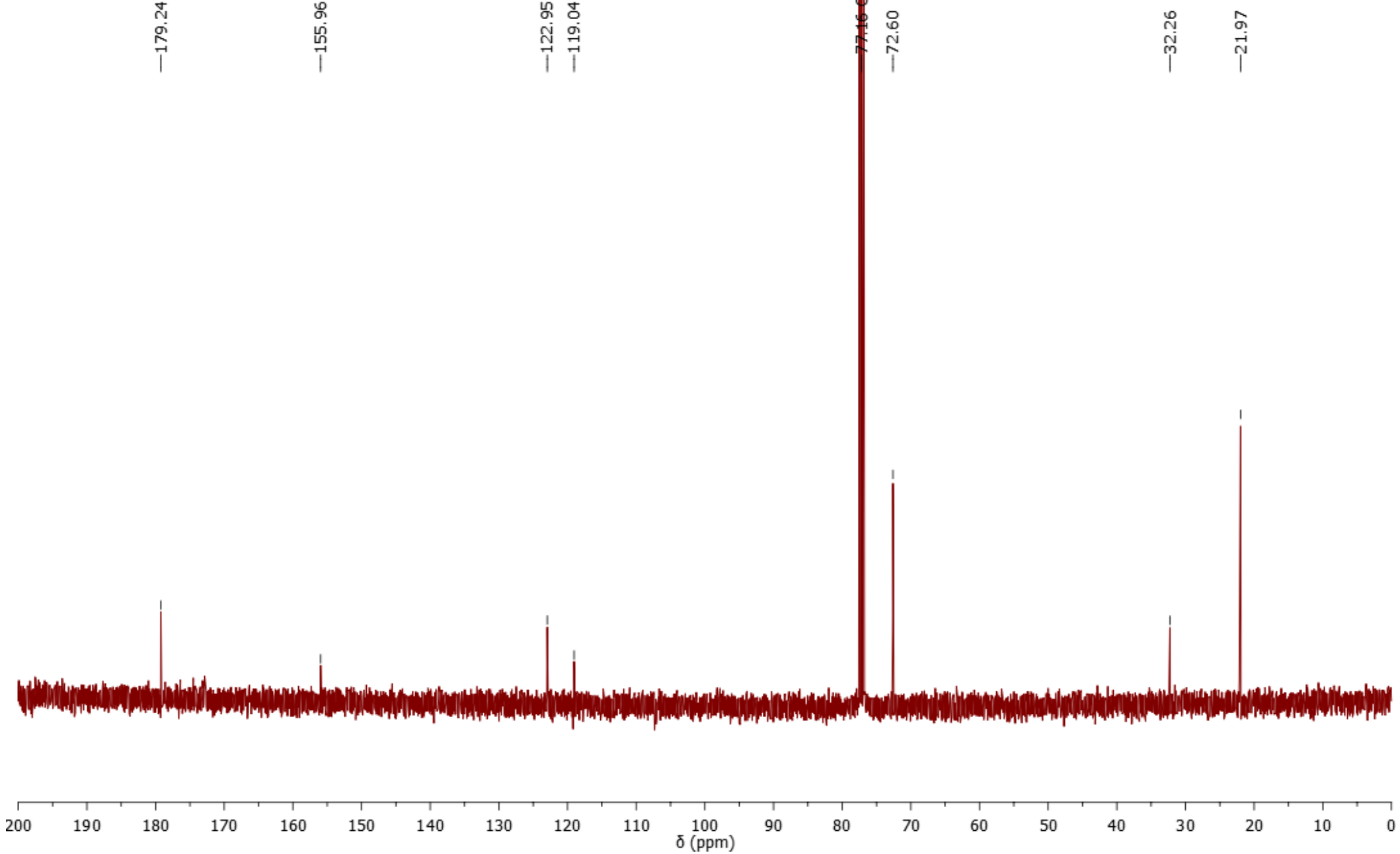

S116 
Furan boronate esters

${ }^{1} \mathrm{H} 400 \mathrm{MHz}, \mathrm{CDCl}_{3}$
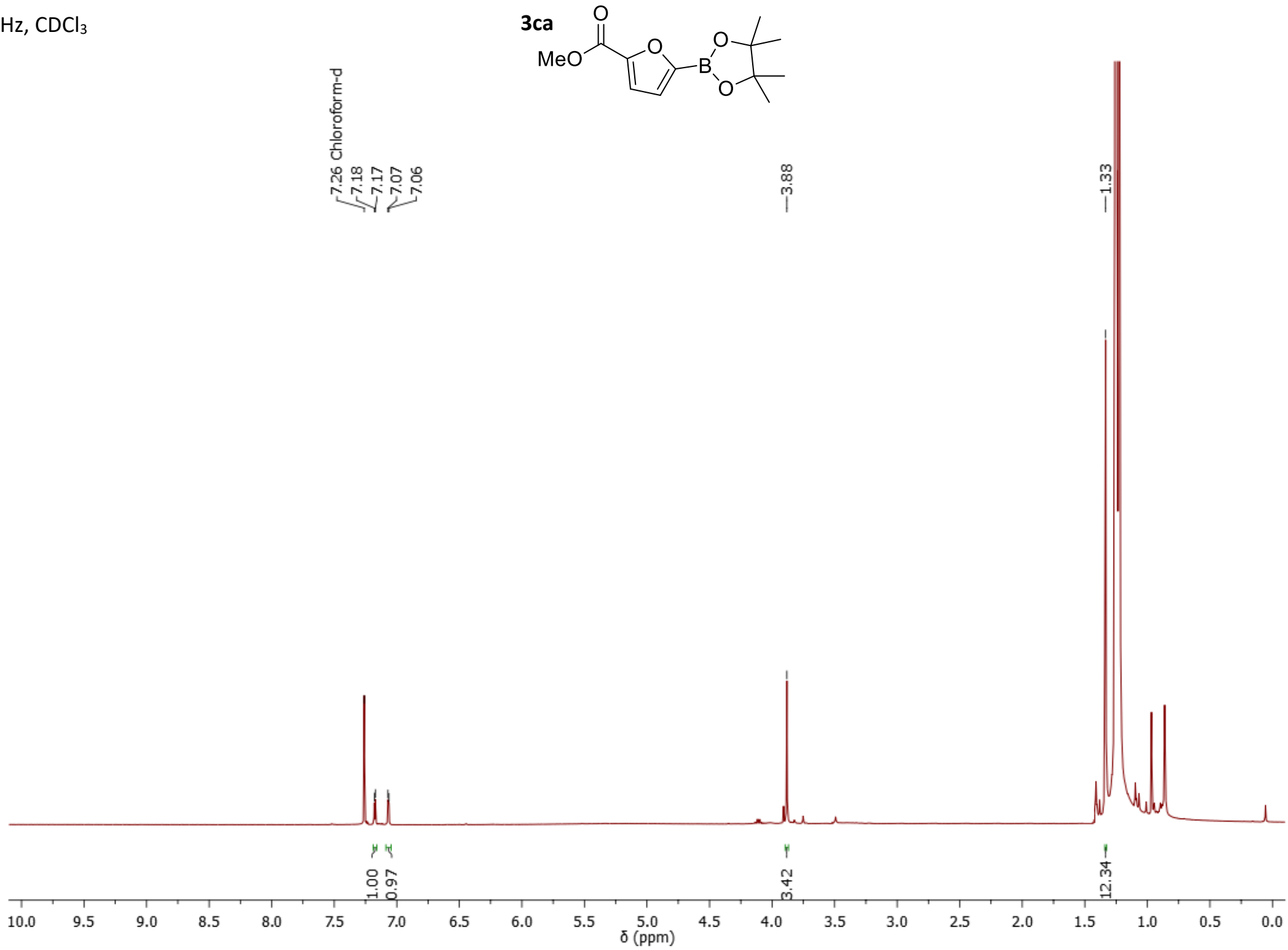
Furan boronate esters

${ }^{13} \mathrm{C} 101 \mathrm{MHz}, \mathrm{CDCl}_{3}$
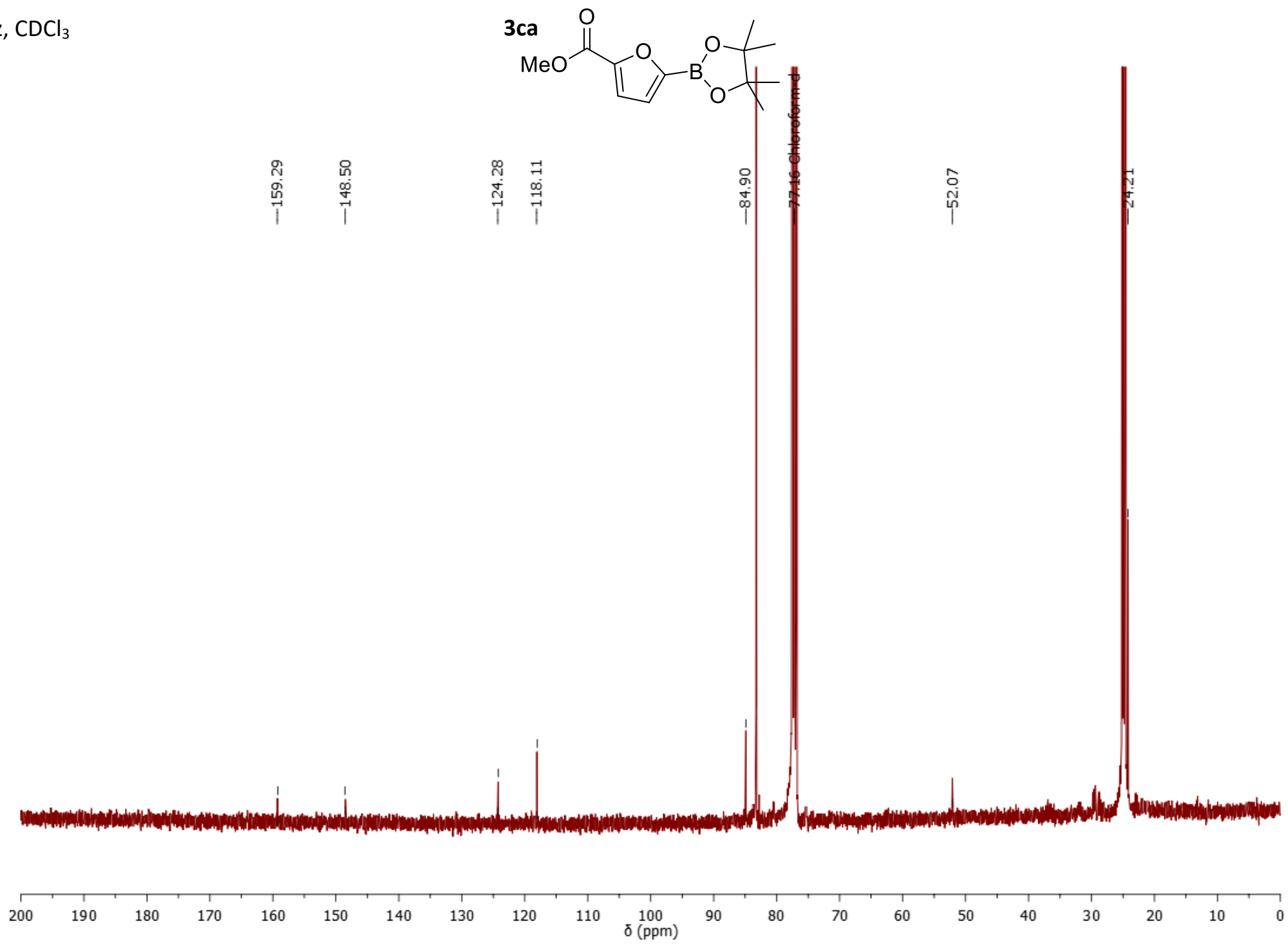

S118 
Furan boronate esters

${ }^{1} \mathrm{H} 400 \mathrm{MHz}, \mathrm{CDCl}_{3}$

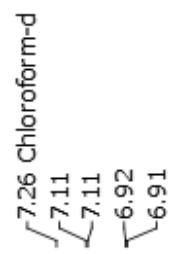

$3 c b$

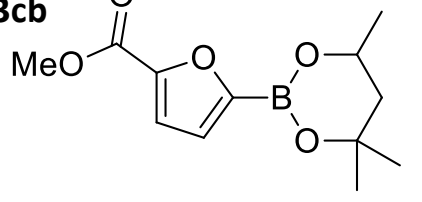

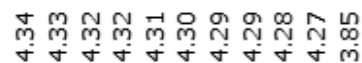

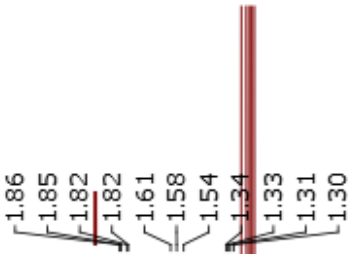

.

过
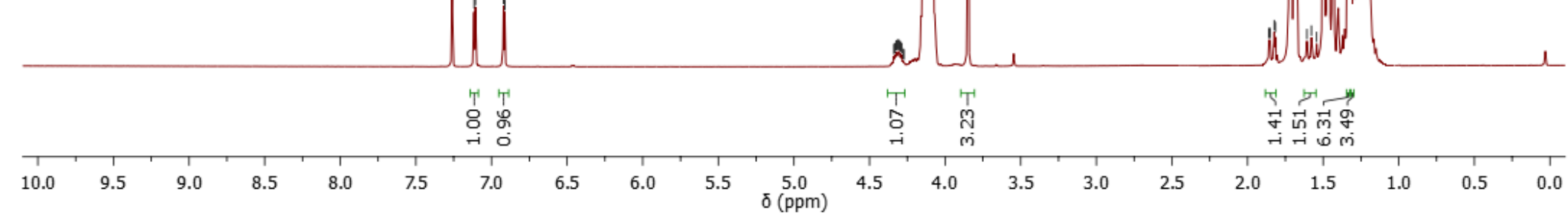


\section{Furan boronate esters}

${ }^{13} \mathrm{C} 101 \mathrm{MHz}$
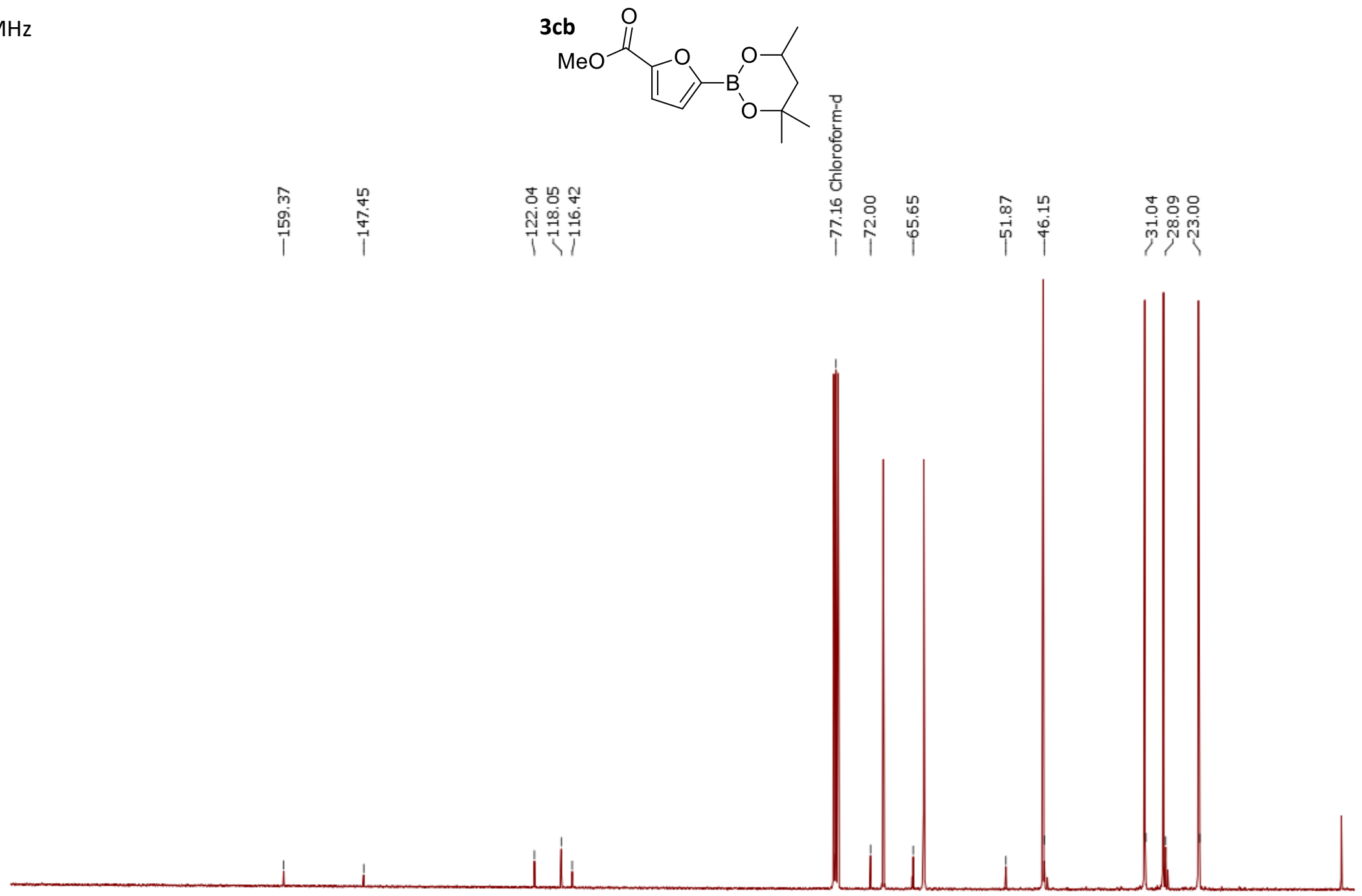

20
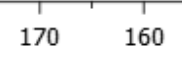

150

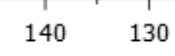

120
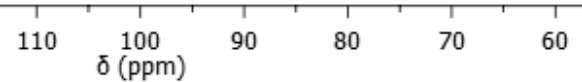


\section{Furan boronate esters}

${ }^{1} \mathrm{H} 400 \mathrm{MHz}, \mathrm{CDCl}_{3}$

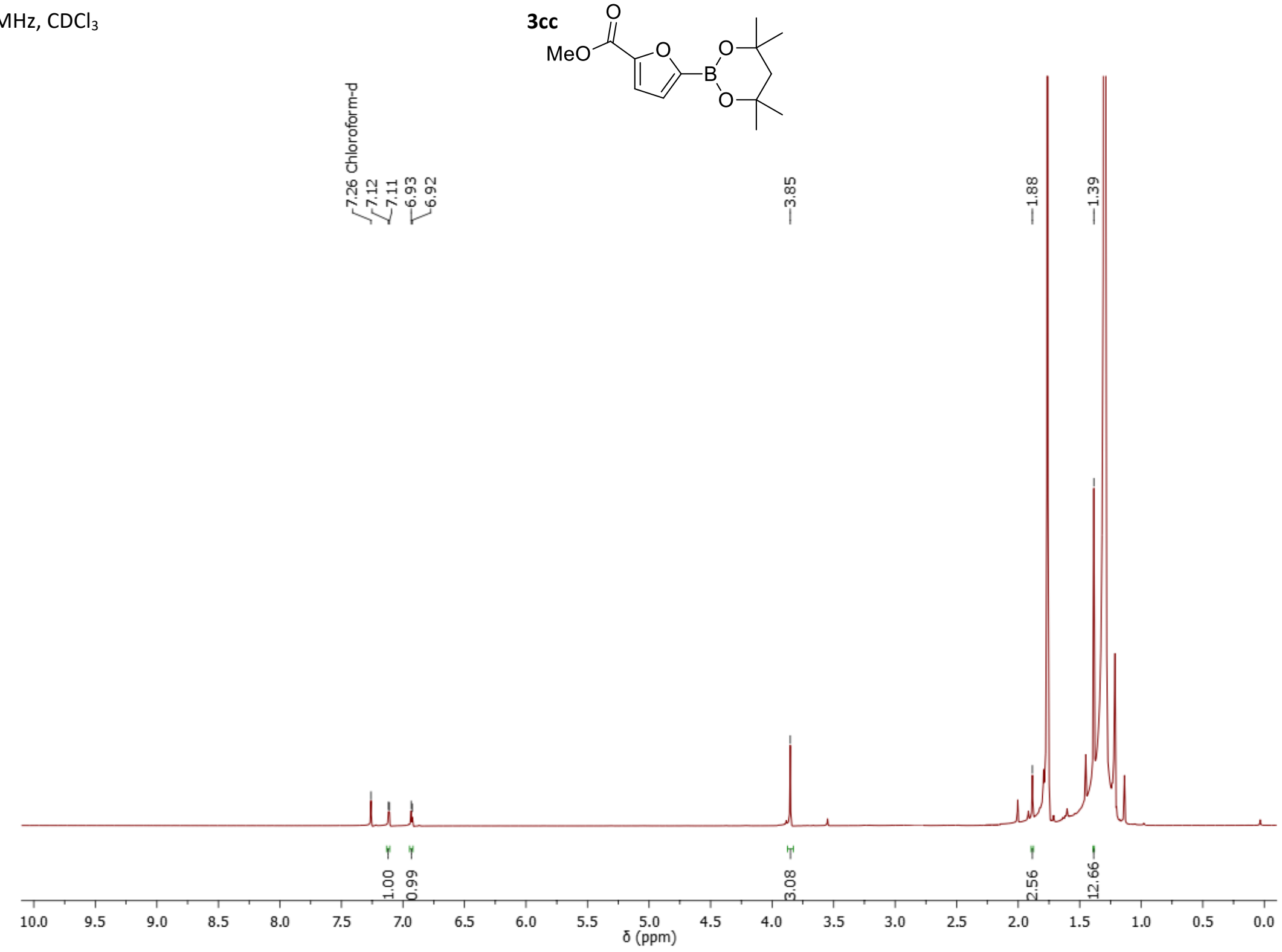


Furan boronate esters

${ }^{13} \mathrm{C} 101 \mathrm{MHz}$

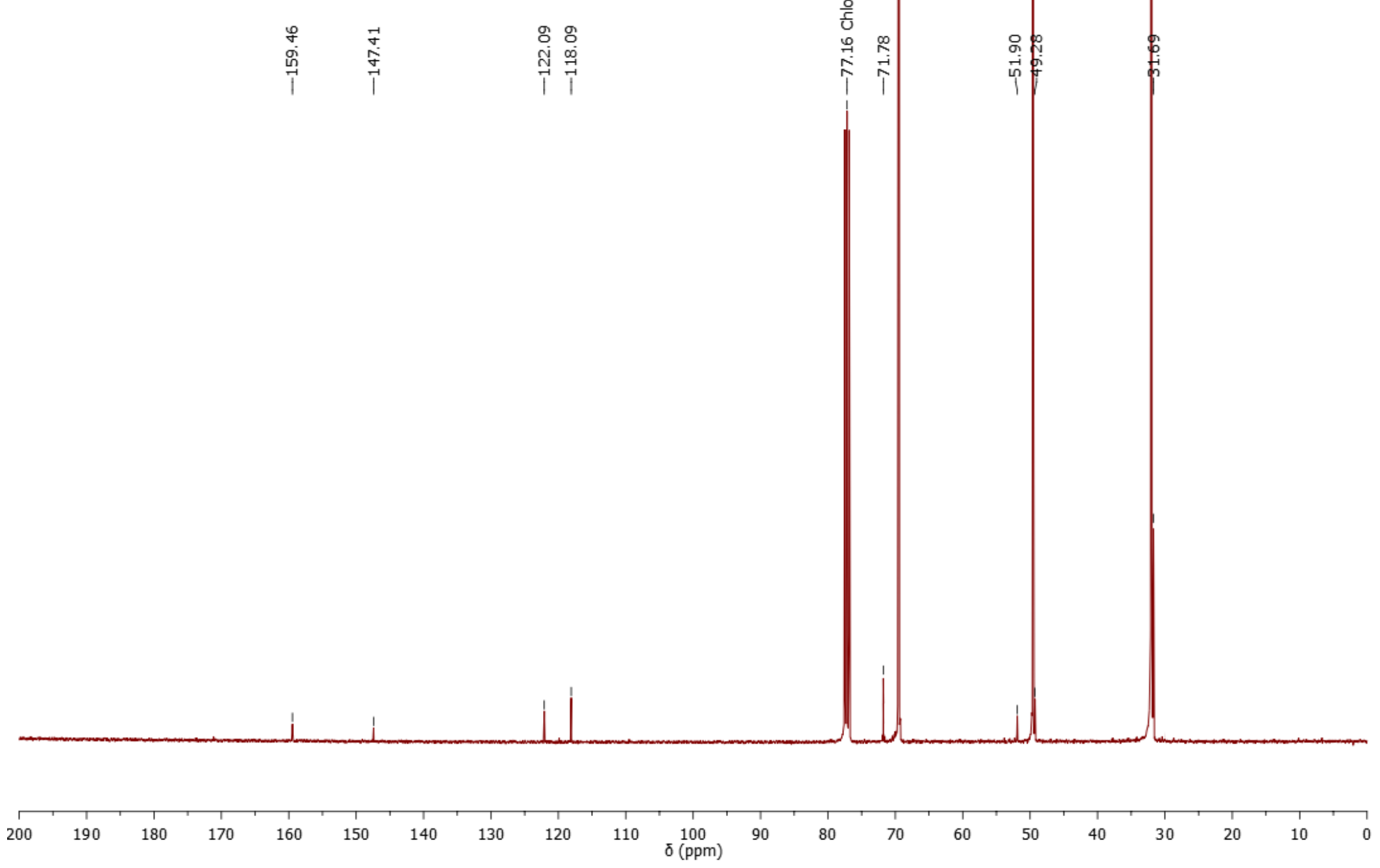

S122 


\section{Furan boronate esters}

${ }^{1} \mathrm{H} 400 \mathrm{MHz}, \mathrm{CDCl}_{3}$

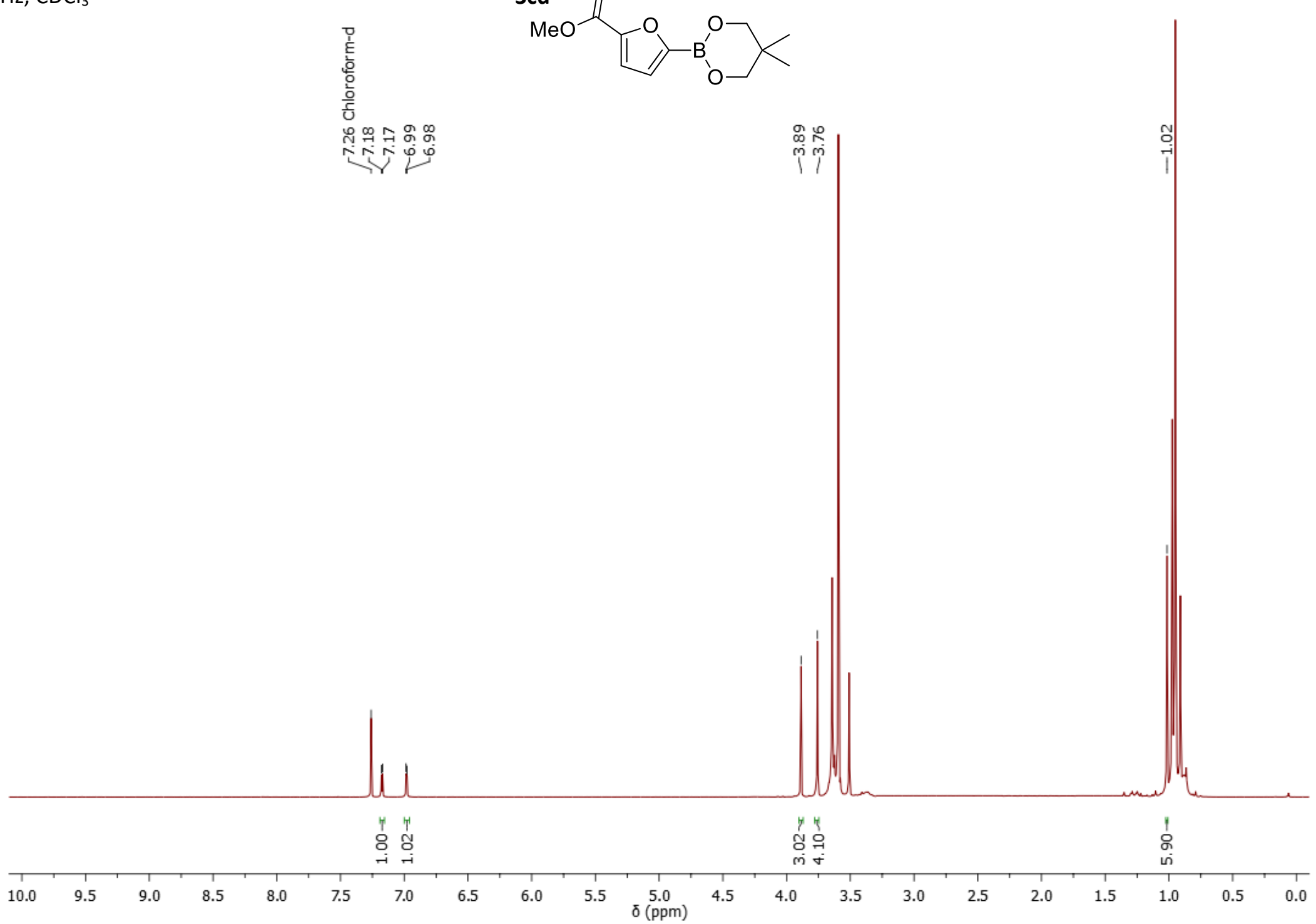


Furan boronate esters

${ }^{13} \mathrm{C} 101 \mathrm{MHz}$

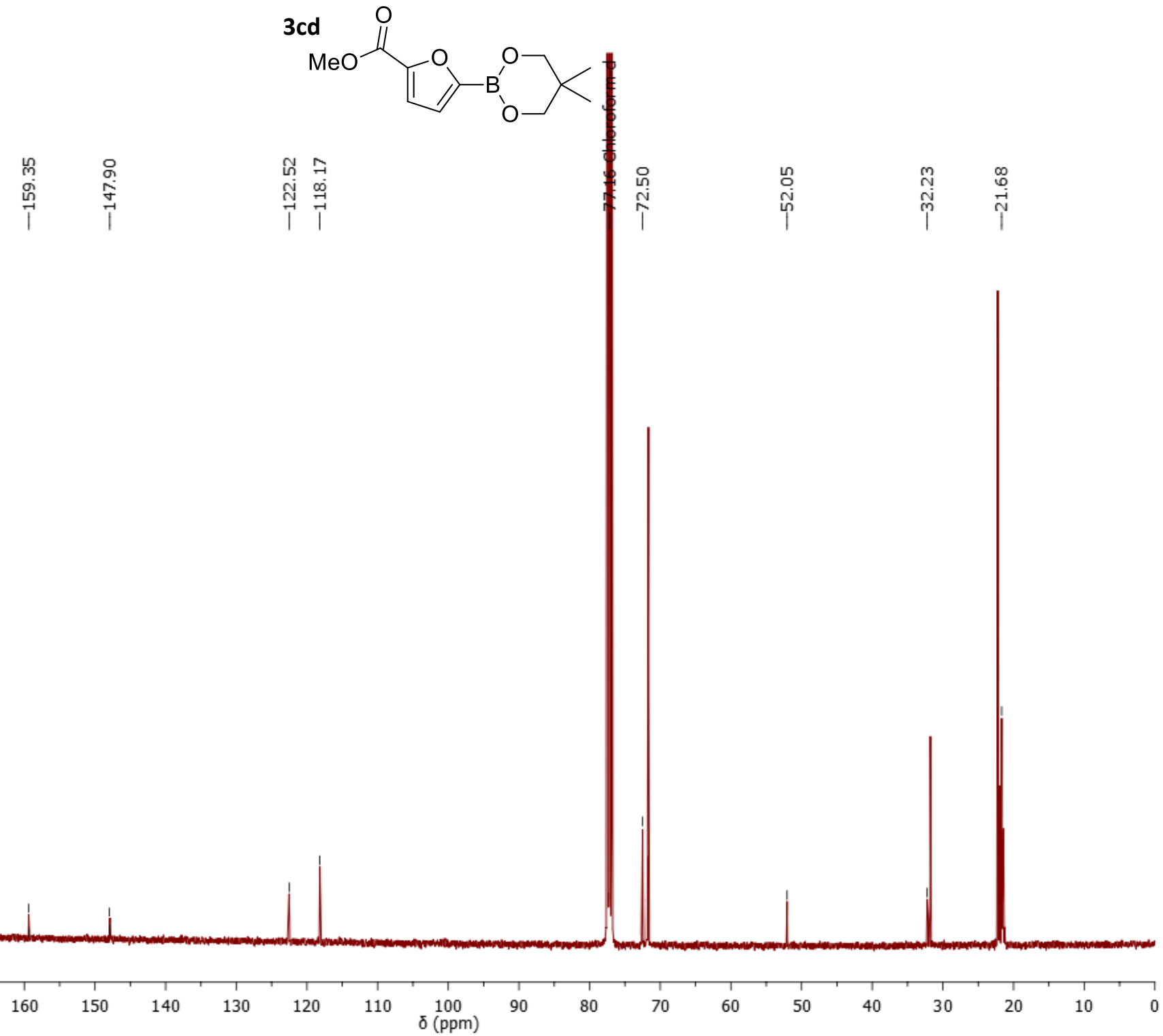


$\underline{\text { Selenophene boronate esters }}$

${ }^{1} \mathrm{H} 400 \mathrm{MHz}, \mathrm{CDCl}_{3}$

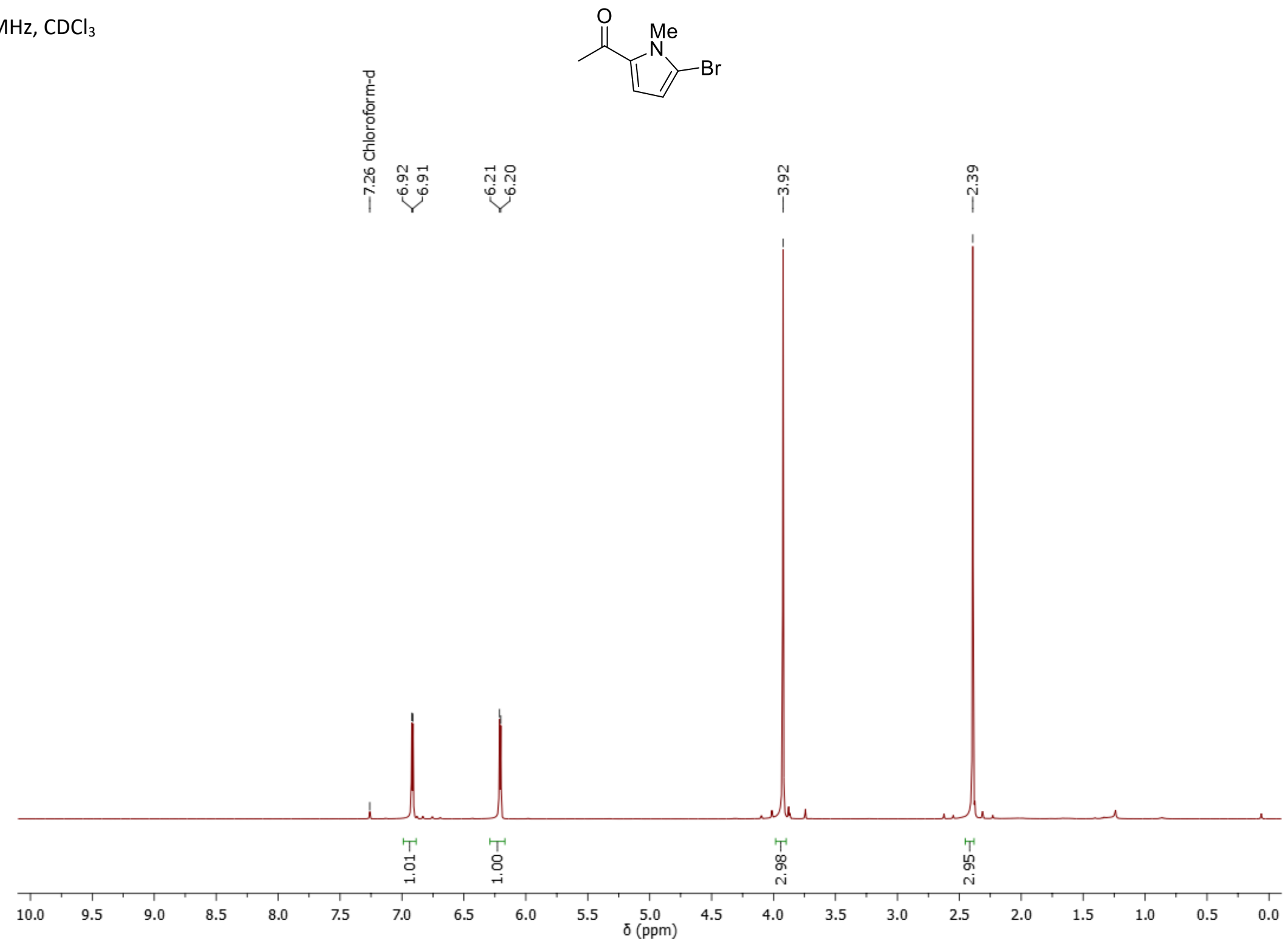


$\underline{\text { Selenophene boronate esters }}$

${ }^{13} \mathrm{C} 101 \mathrm{MHz}, \mathrm{CDCl}_{3}$
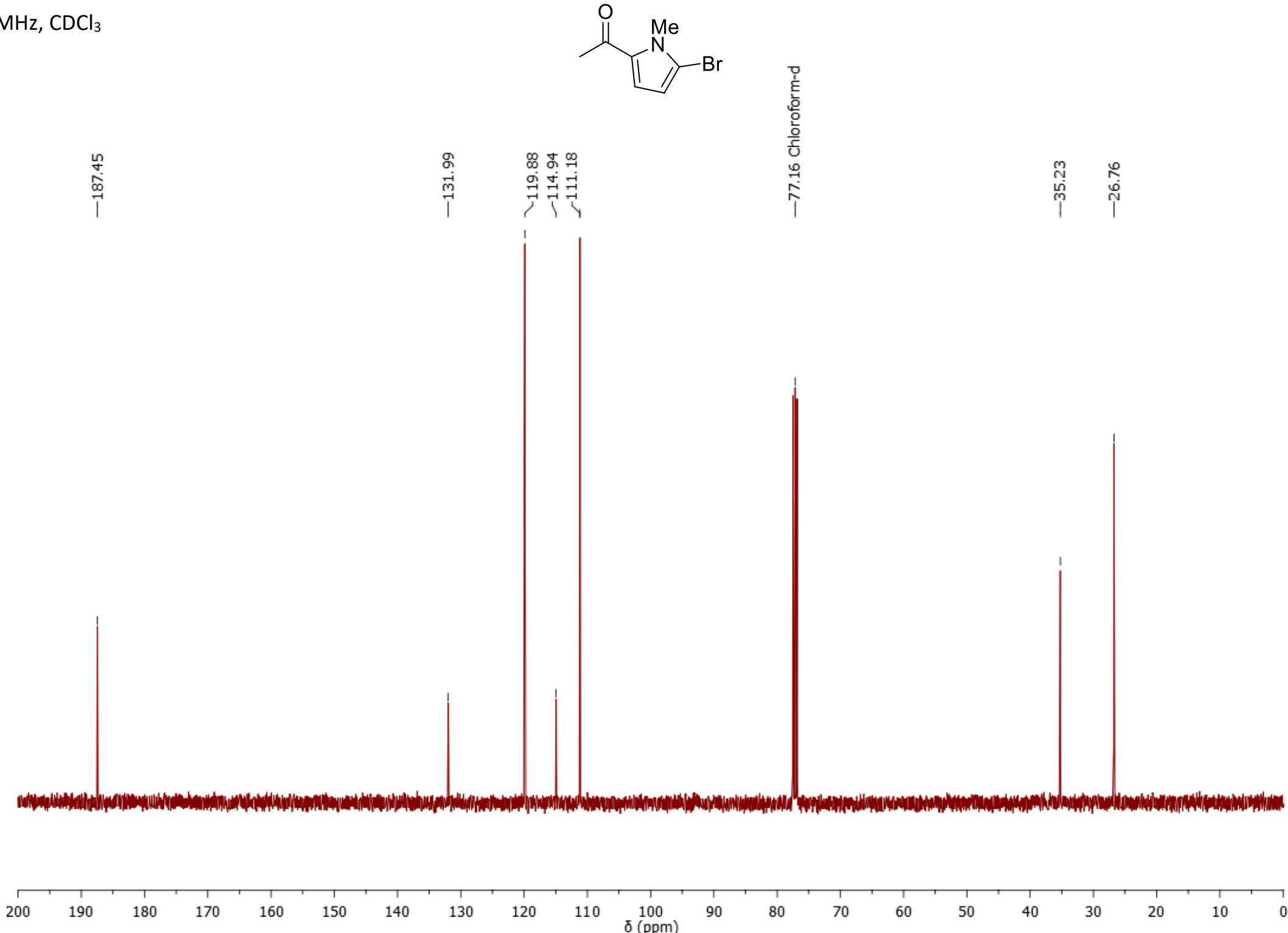
$\underline{\text { Selenophene boronate esters }}$

${ }^{1} \mathrm{H} 400 \mathrm{MHz}, \mathrm{CDCl}_{3}$
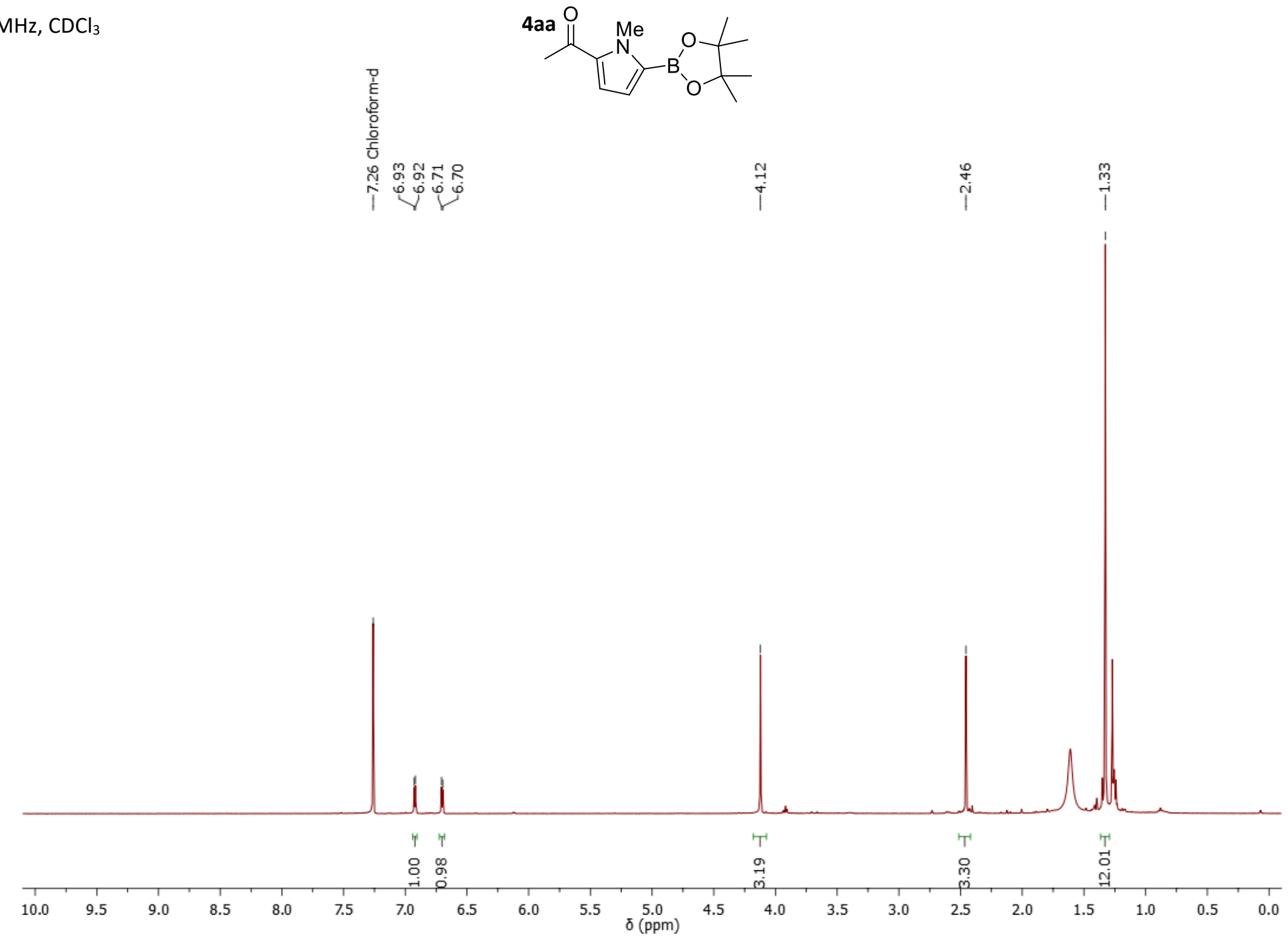
$\underline{\text { Selenophene boronate esters }}$

${ }^{13} \mathrm{C} 101 \mathrm{MHz}, \mathrm{CDCl}_{3}$

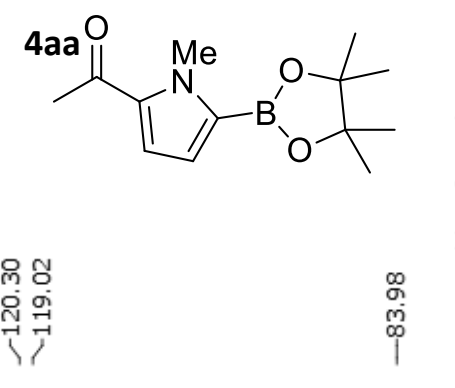

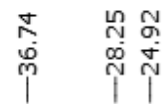

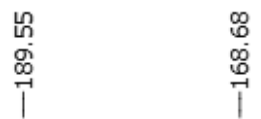

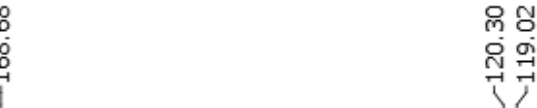

,

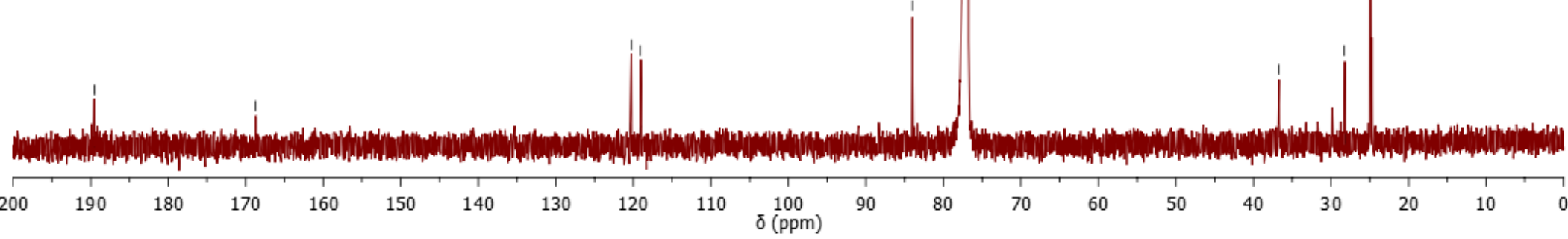


${ }^{1} \mathrm{H} 400 \mathrm{MHz}, \mathrm{CDCl}_{3}$
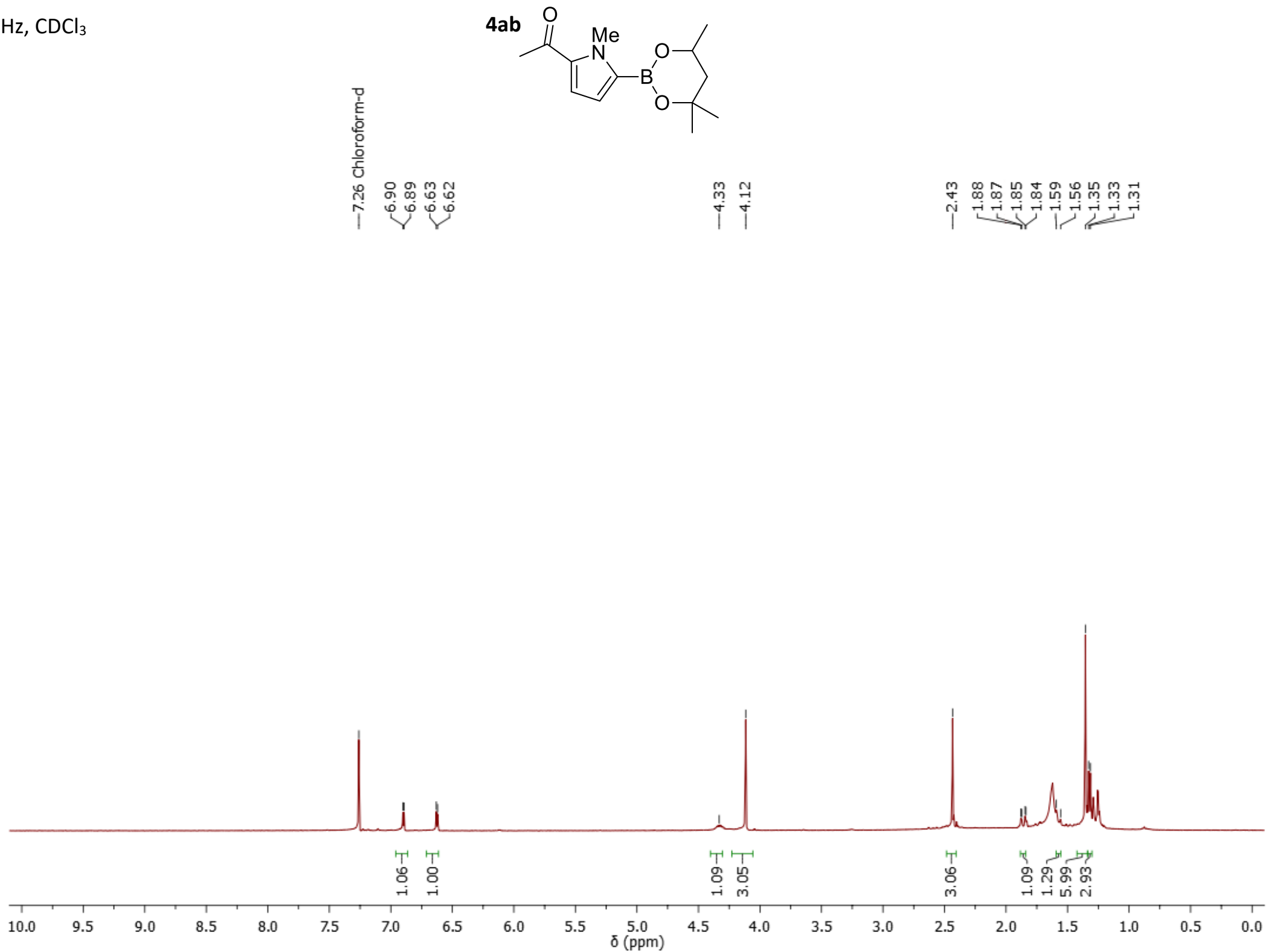
Selenophene boronate esters

${ }^{13} \mathrm{C} 101 \mathrm{MHz}$

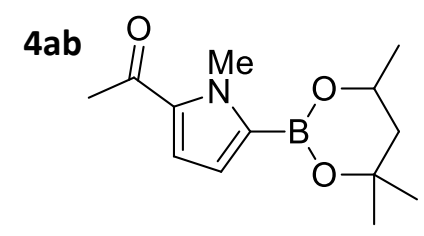

90
9.9
90
$7=0$
$\gamma$

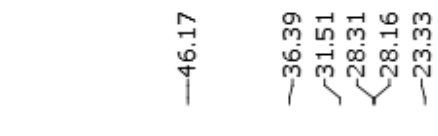

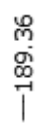

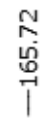

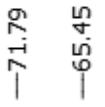
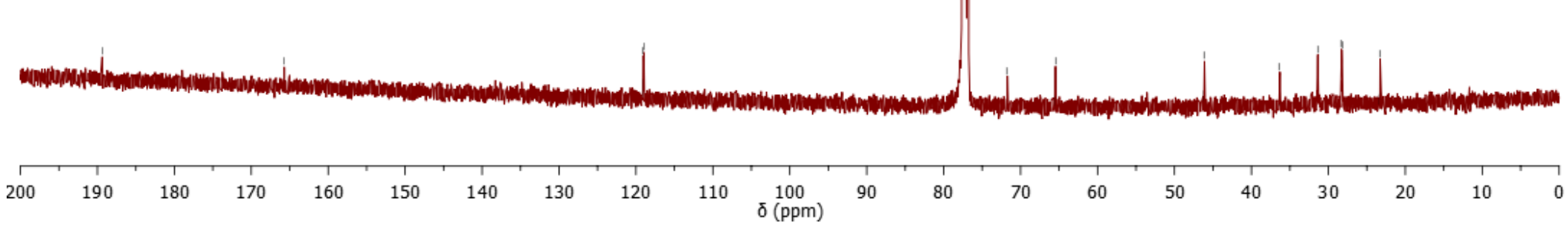

S130 
${ }^{1} \mathrm{H} 400 \mathrm{MHz}, \mathrm{CDCl}_{3}$

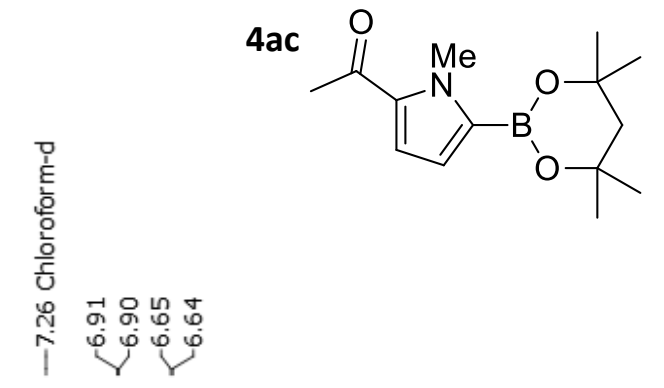

$\frac{1}{d_{1}^{2}}$

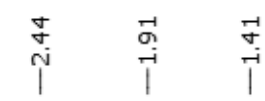

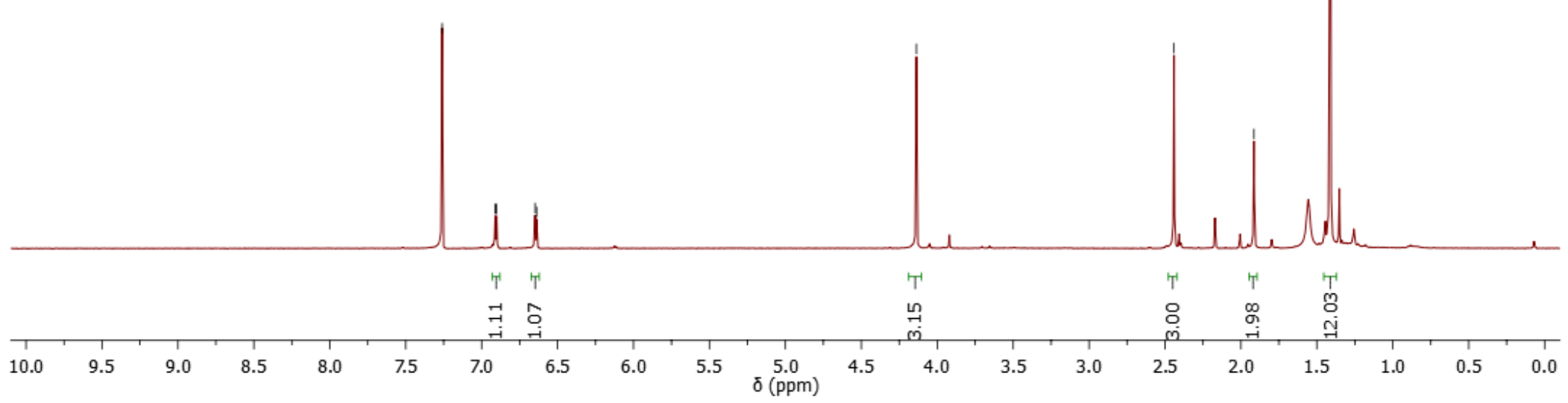


Selenophene boronate esters

${ }^{13} \mathrm{C} 101 \mathrm{MHz}$

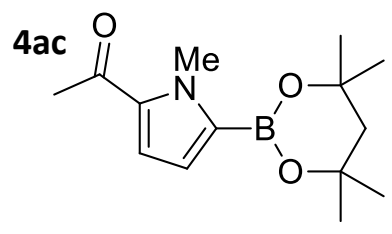

ํㅣㄹ

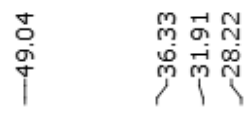

S132 
$\underline{\text { Selenophene boronate esters }}$

${ }^{1} \mathrm{H} 400 \mathrm{MHz}, \mathrm{CDCl}_{3}$

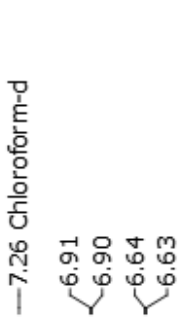

4ad
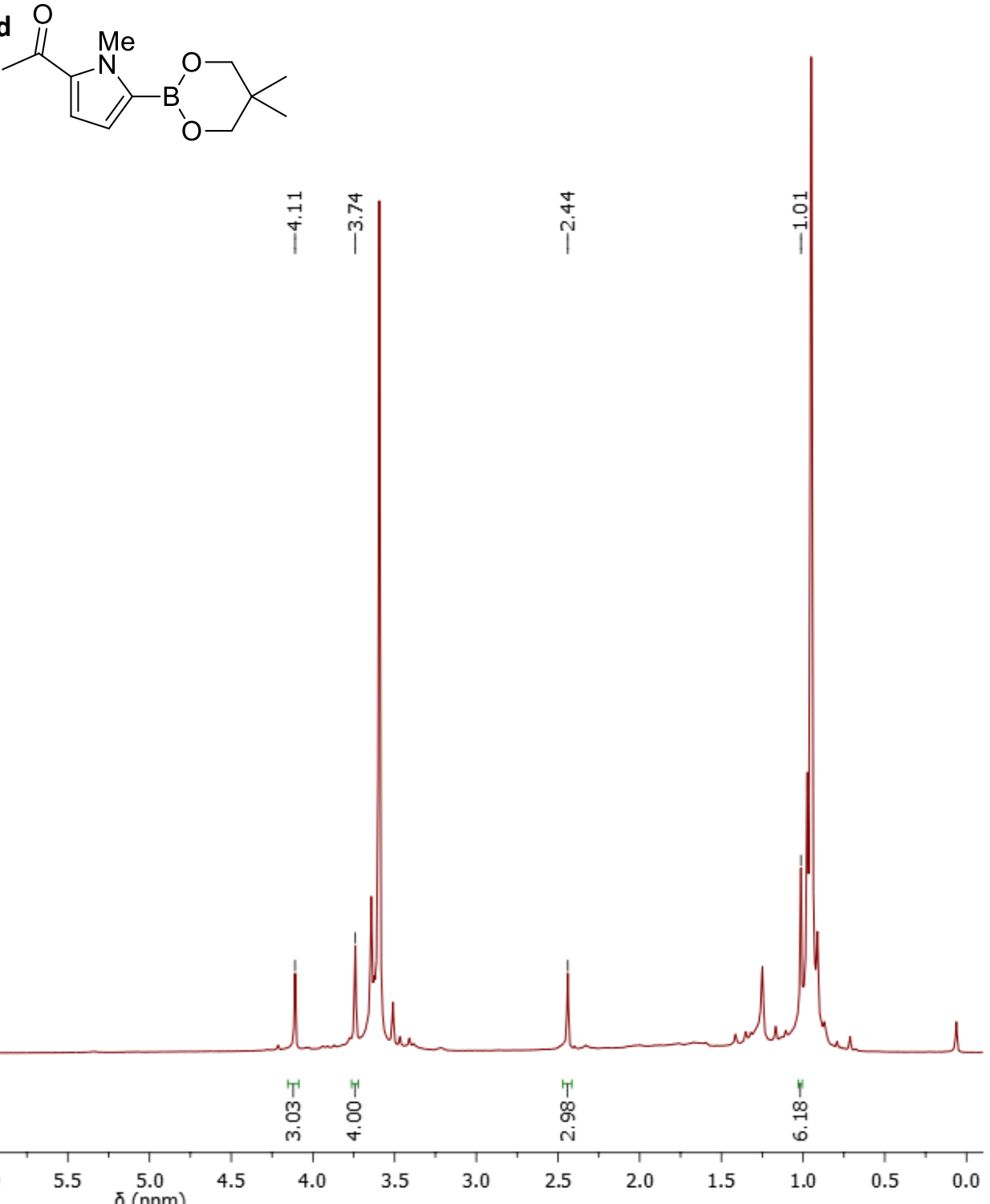
$\underline{\text { Selenophene boronate esters }}$

${ }^{13} \mathrm{C} 101 \mathrm{MHz}$

4ad

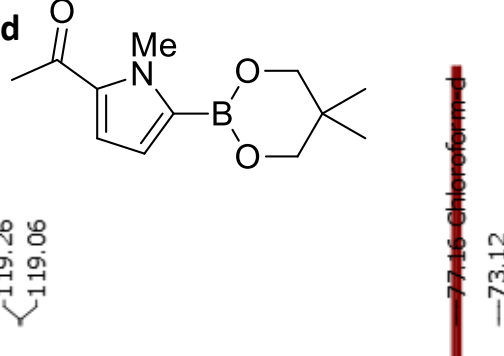

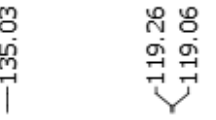

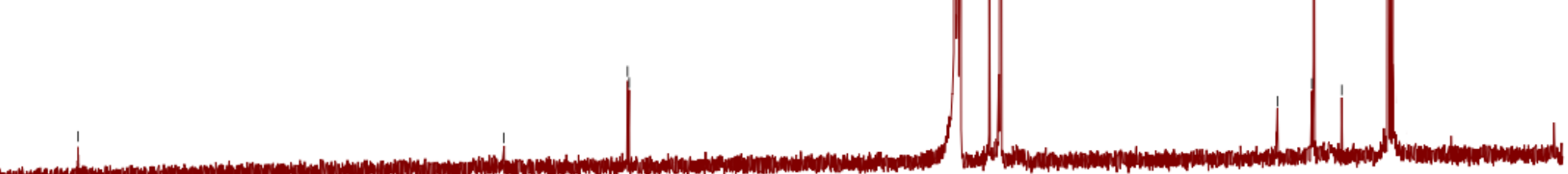


$\underline{\text { Selenophene boronate esters }}$

${ }^{1} \mathrm{H} 400 \mathrm{MHz}, \mathrm{CDCl}_{3}$
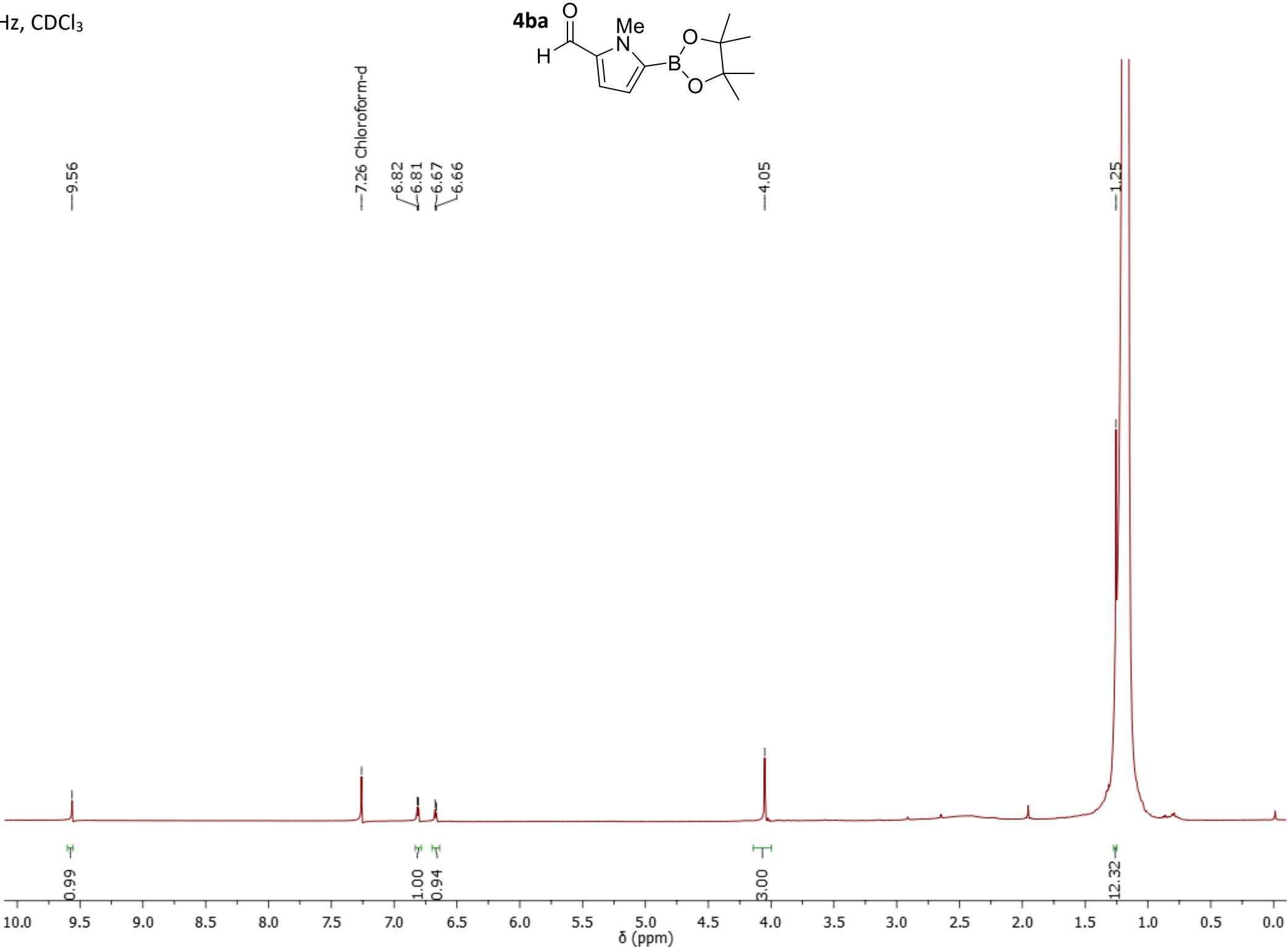
$\underline{\text { Selenophene boronate esters }}$

${ }^{13} \mathrm{C} 101 \mathrm{MHz}, \mathrm{CDCl}_{3}$

4ba

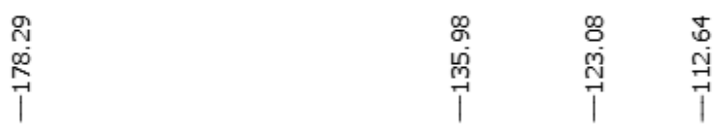

200

$190 \quad 180$

170

160

$150 \quad 140$

130

120

100
$\delta(\mathrm{ppm})$

80

70

60

50

$40 \quad 3$

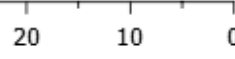


${ }^{1} \mathrm{H} 400 \mathrm{MHz}, \mathrm{CDCl}_{3}$

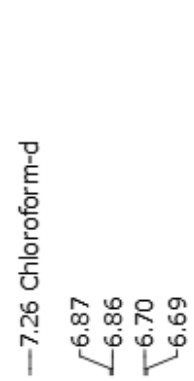

4bb

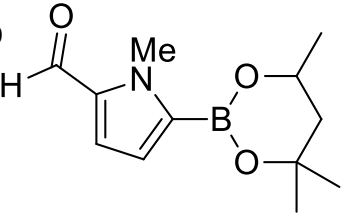

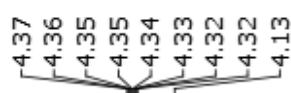

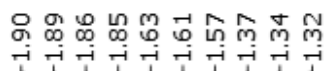

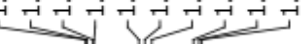

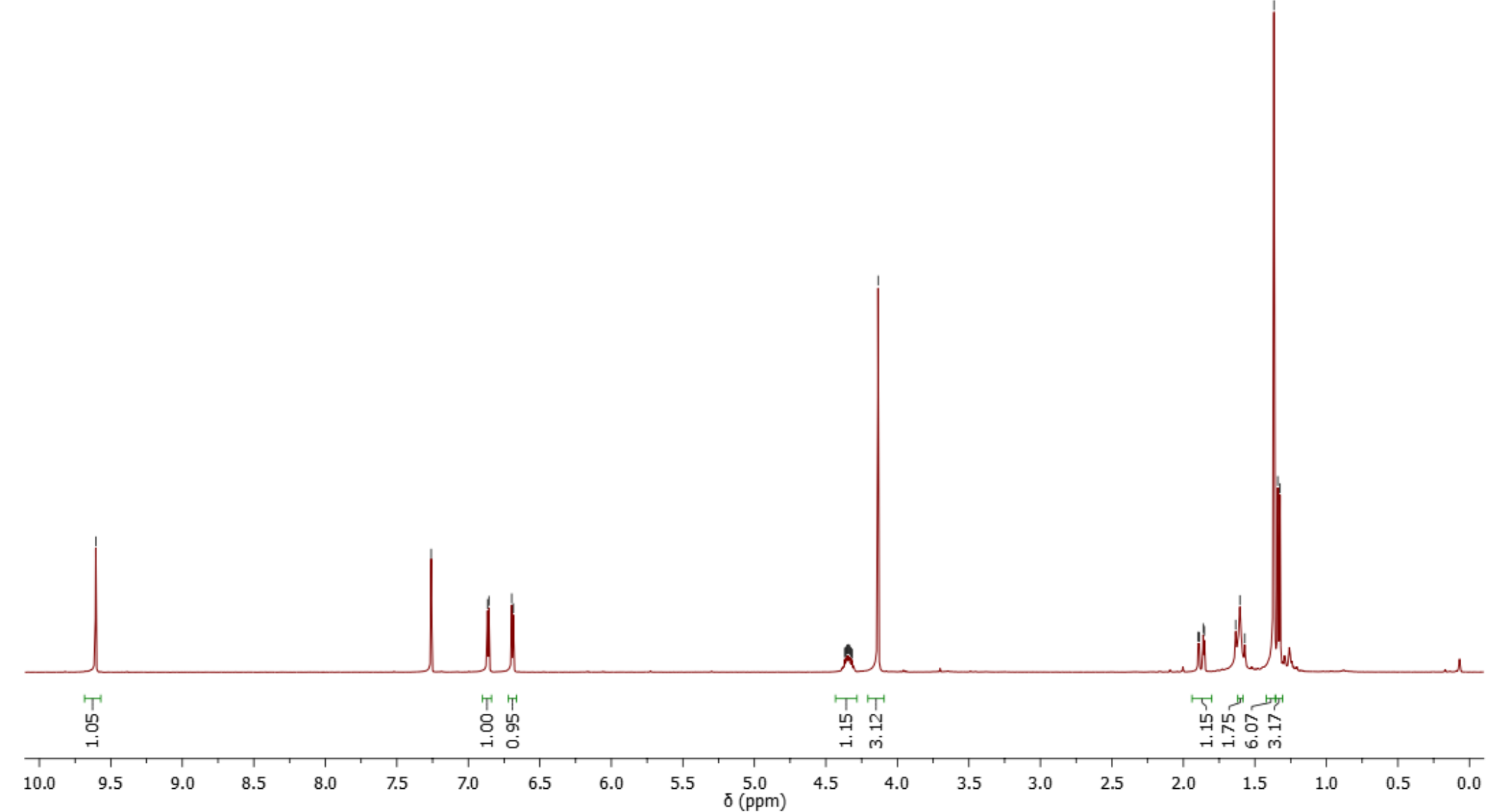


Selenophene boronate esters

${ }^{13} \mathrm{C} 101 \mathrm{MHz}$

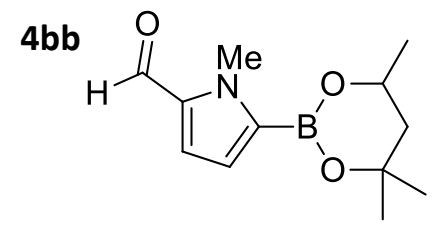

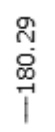

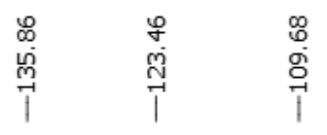
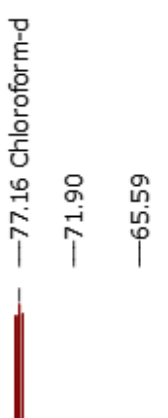

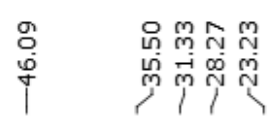

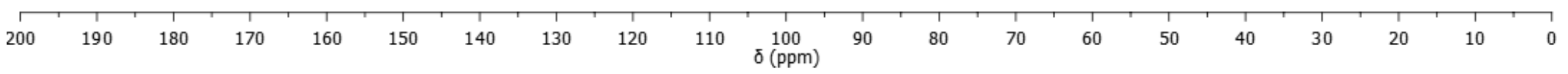

S138 
$\underline{\text { Selenophene boronate esters }}$

${ }^{1} \mathrm{H} 400 \mathrm{MHz}, \mathrm{CDCl}_{3}$

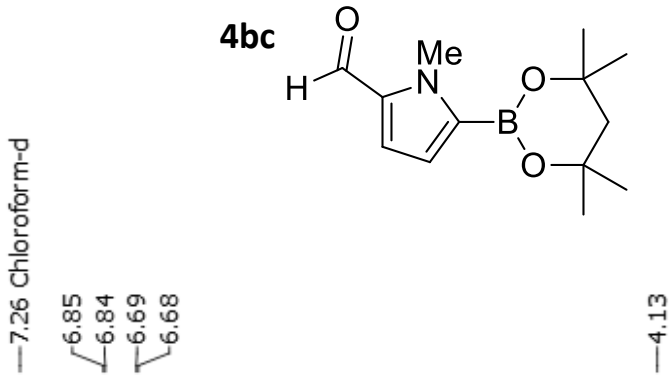

$\stackrel{m}{\underset{j}{\dagger}}$

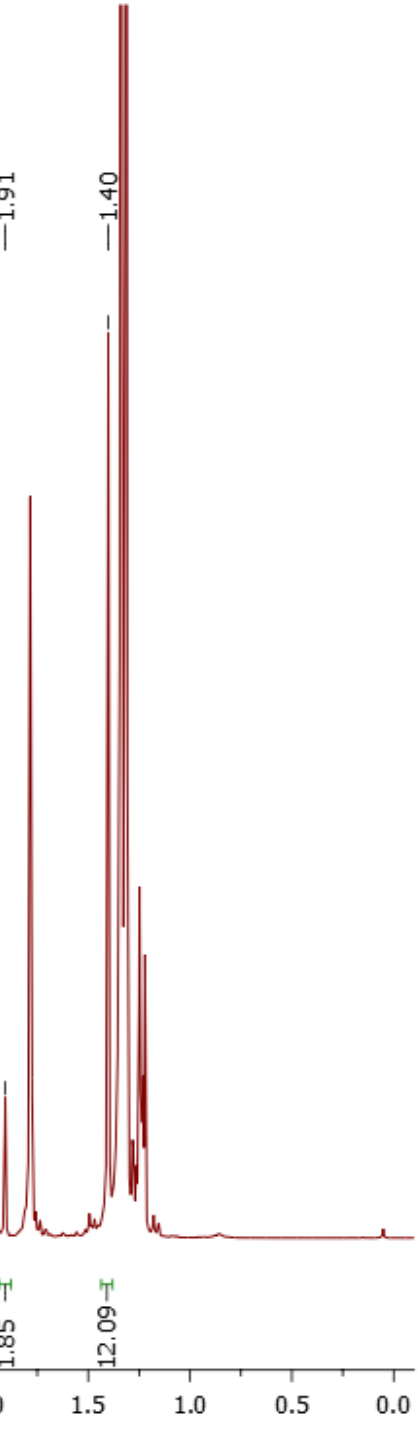


$\underline{\text { Selenophene boronate esters }}$

${ }^{13} \mathrm{C} 101 \mathrm{MHz}$

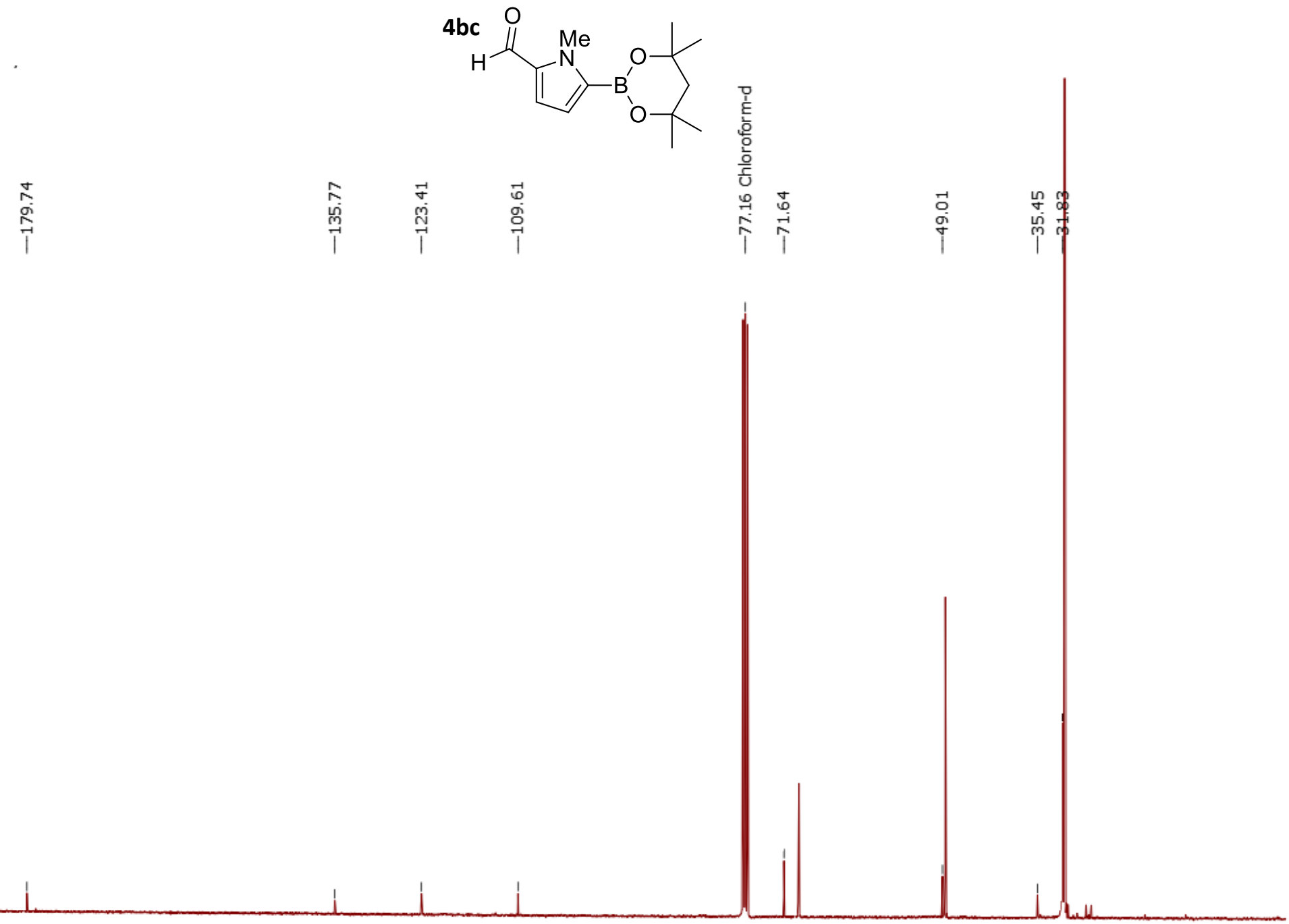

$200 \quad 190 \quad 180$

160

$50 \quad 140$

$130 \quad 120$

100

90
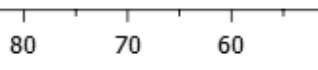

$40 \quad 30$

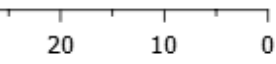


$\underline{\text { Selenophene boronate esters }}$

${ }^{1} \mathrm{H} 400 \mathrm{MHz}, \mathrm{CDCl}_{3}$
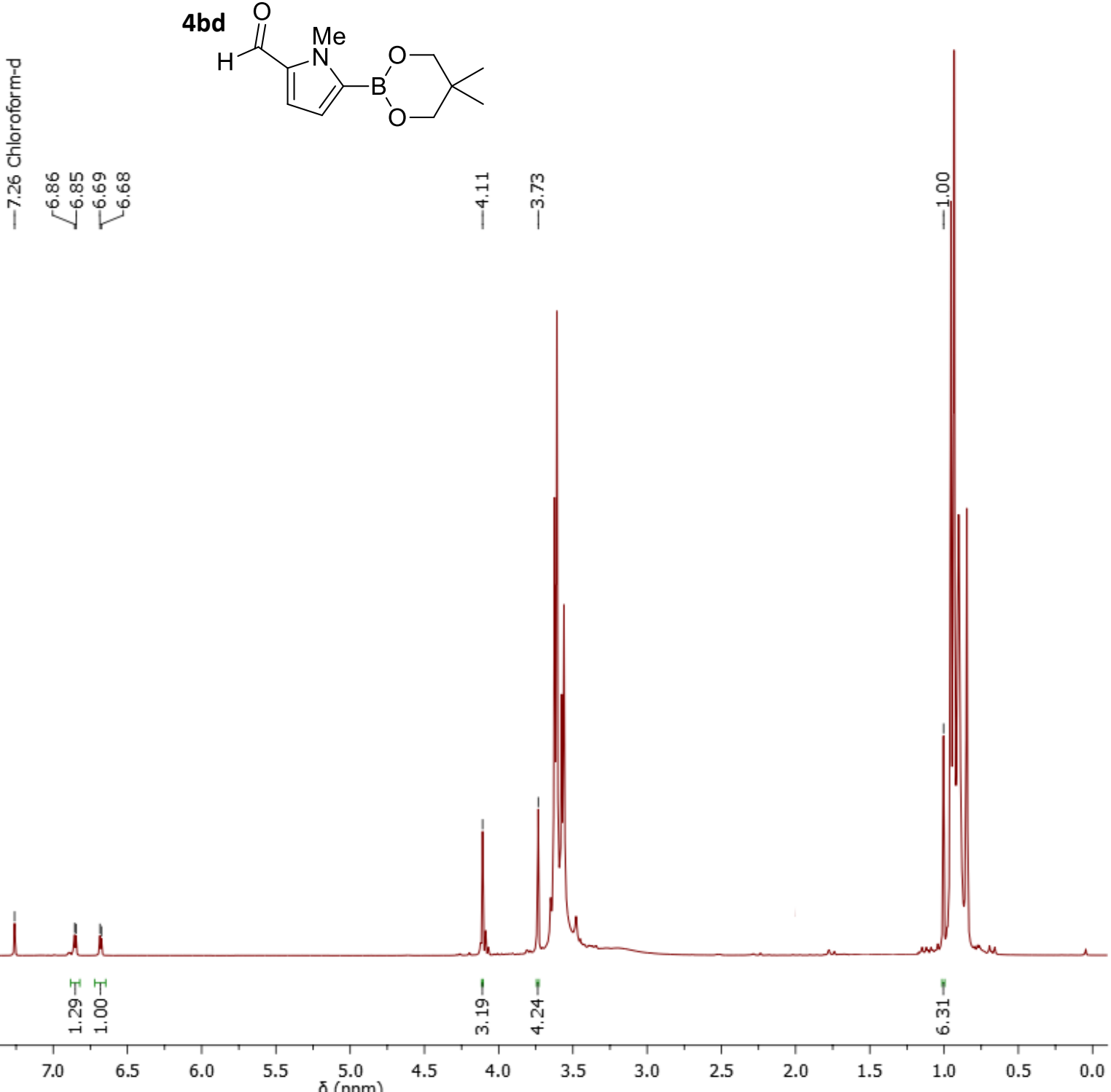
$\underline{\text { Selenophene boronate esters }}$

${ }^{13} \mathrm{C} 101 \mathrm{MHz}$

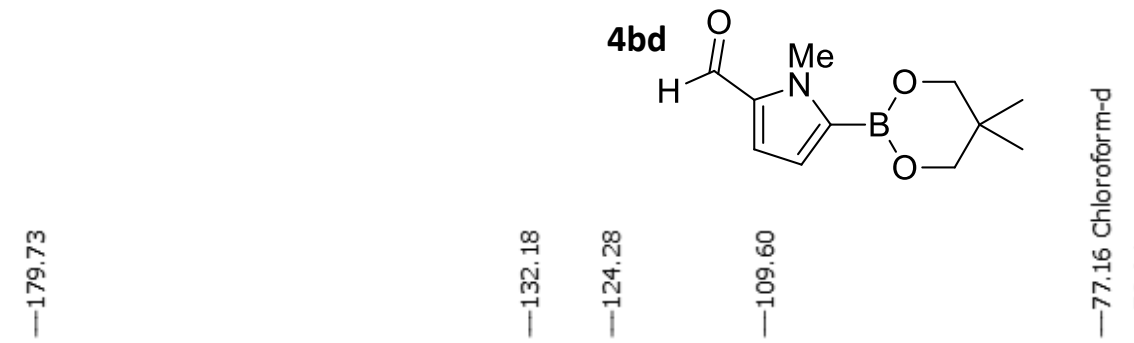

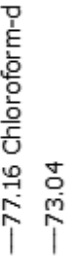

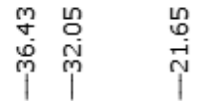

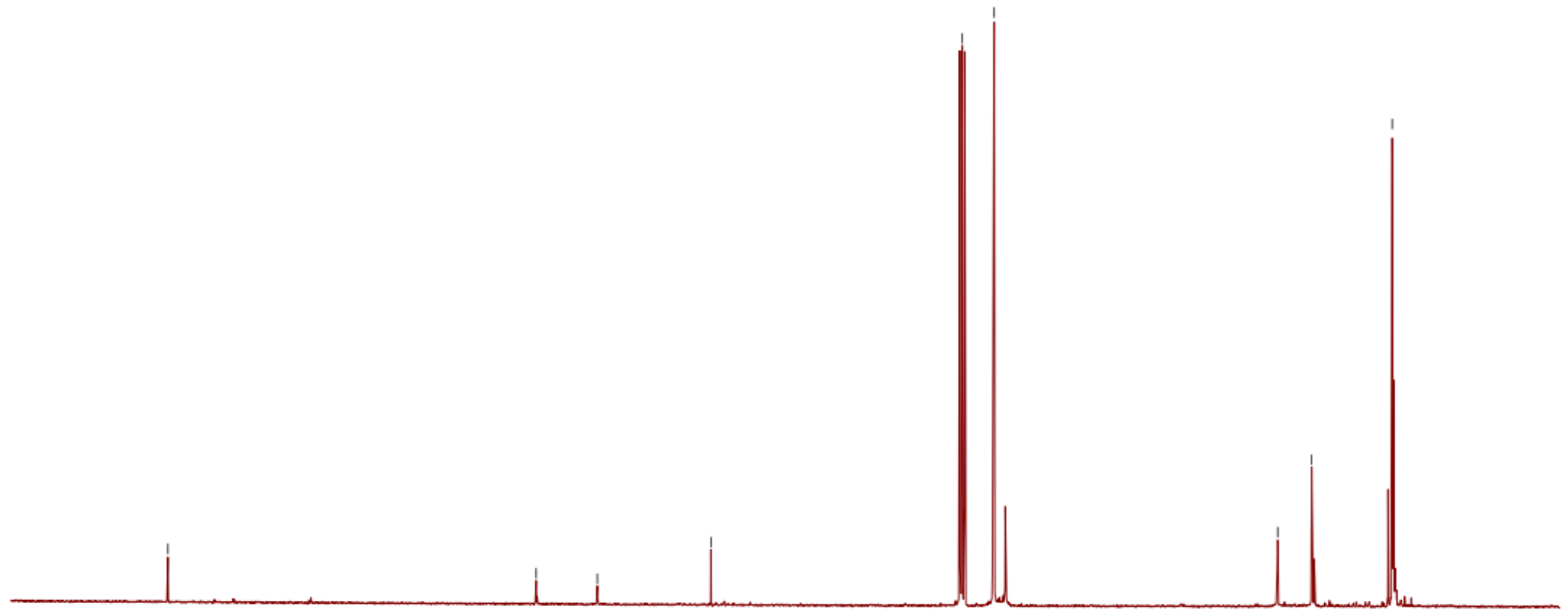

$$
200
$$


$\underline{\text { Selenophene boronate esters }}$

${ }^{1} \mathrm{H} 400 \mathrm{MHz}, \mathrm{CDCl}_{3}$
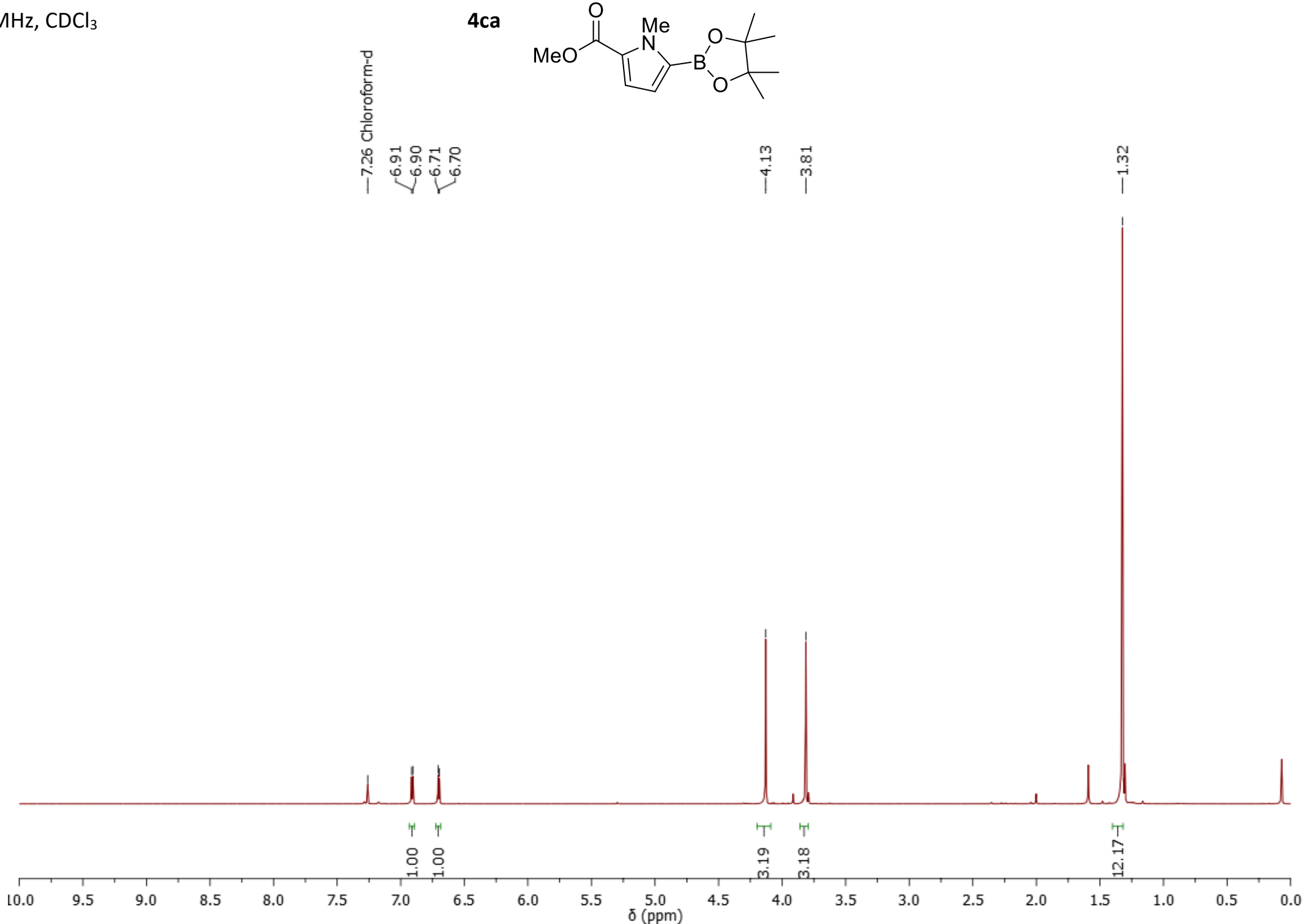
$\underline{\text { Selenophene boronate esters }}$

${ }^{13} \mathrm{C} 101 \mathrm{MHz}, \mathrm{CDCl}_{3}$

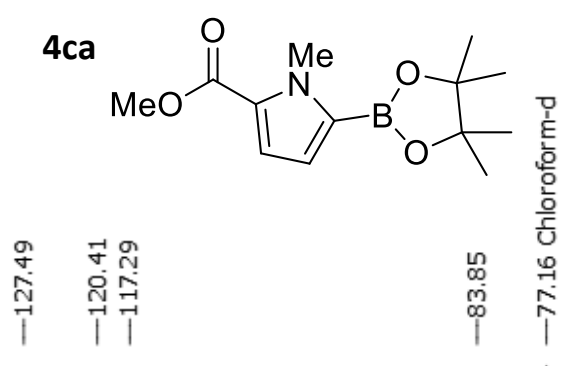

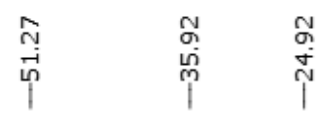

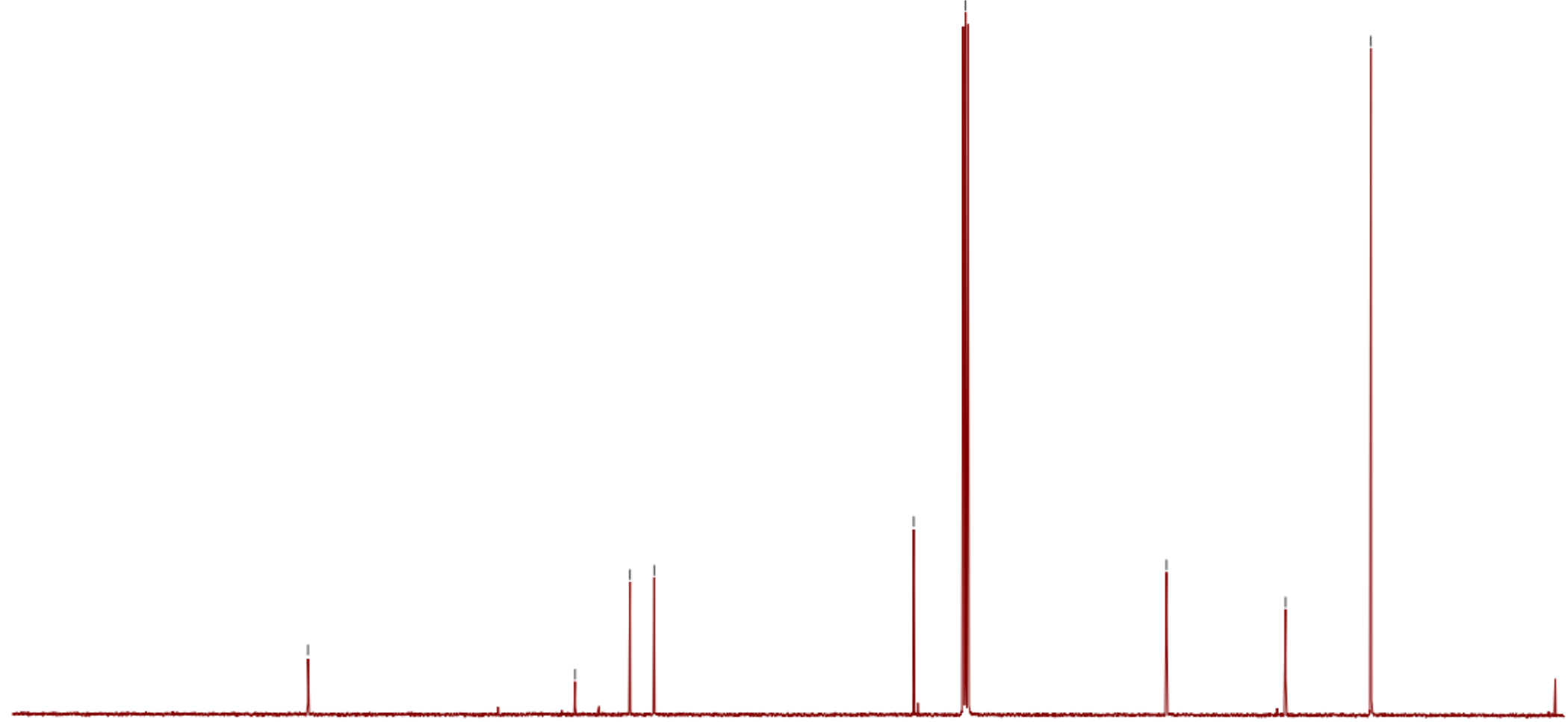

$$
200
$$

190

180

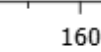

150

140

130

120 $110 \quad \begin{aligned} & 100 \\ & \delta(\mathrm{ppm})\end{aligned}$

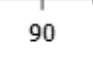

80

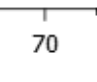

60

50 
$\underline{\text { Selenophene boronate esters }}$

${ }^{1} \mathrm{H} 400 \mathrm{MHz}, \mathrm{CDCl}_{3}$

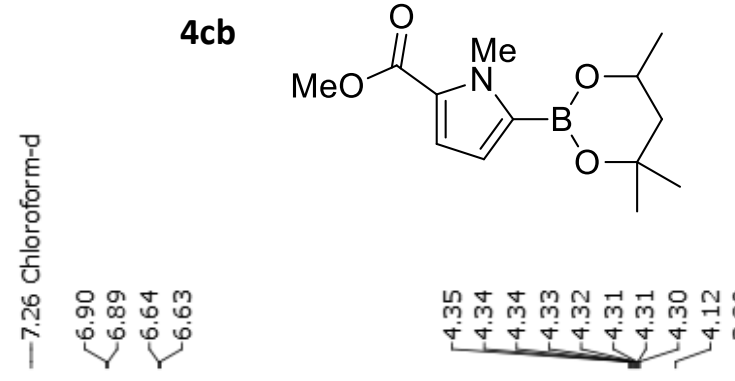

مِ

는

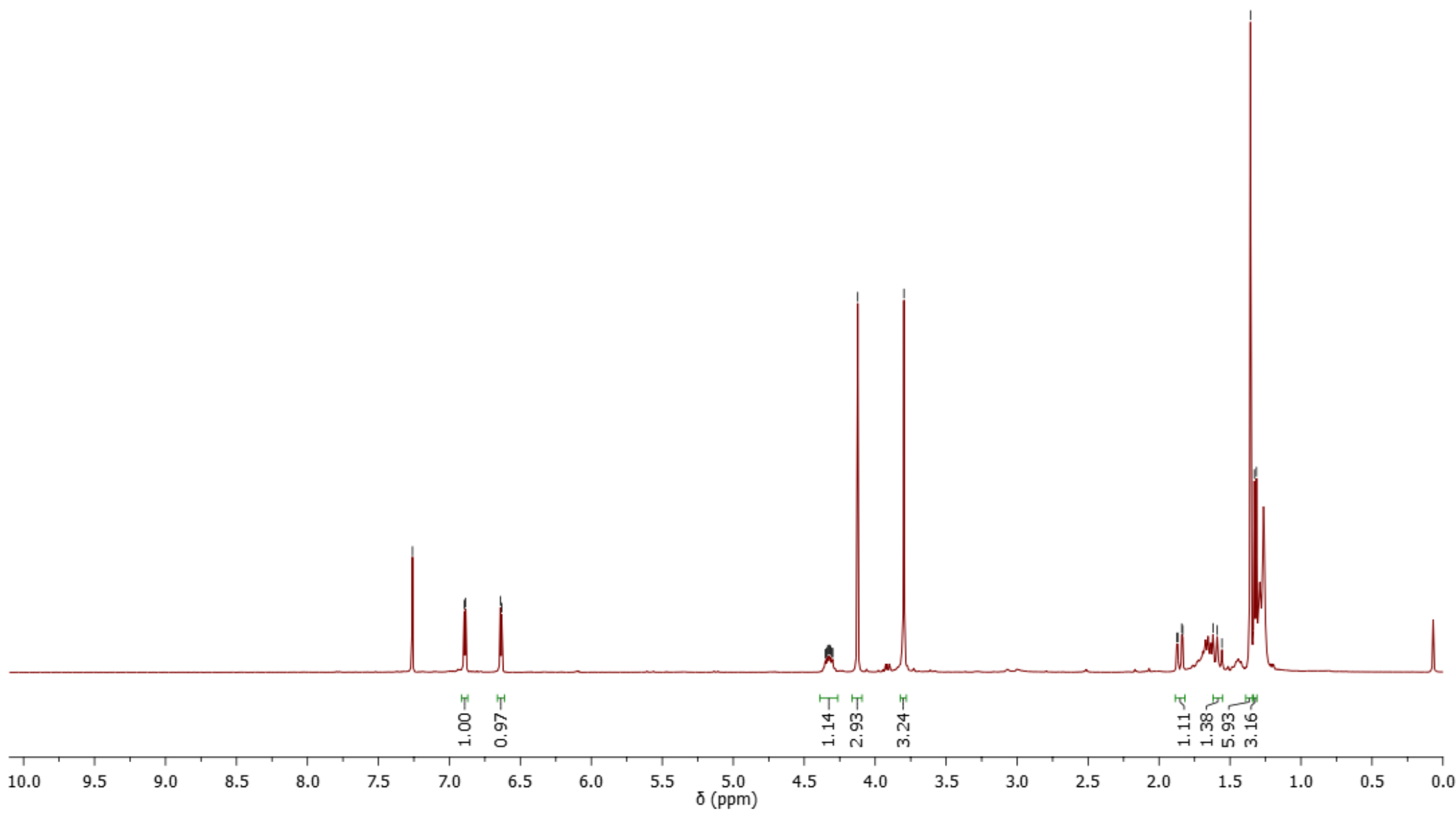


$\underline{\text { Selenophene boronate esters }}$

${ }^{13} \mathrm{C} 101 \mathrm{MHz}$

$4 c b$

我

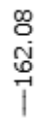

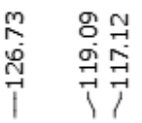

$$
\text { 항 }
$$

남

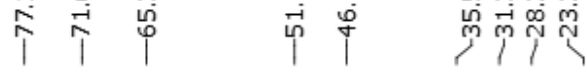

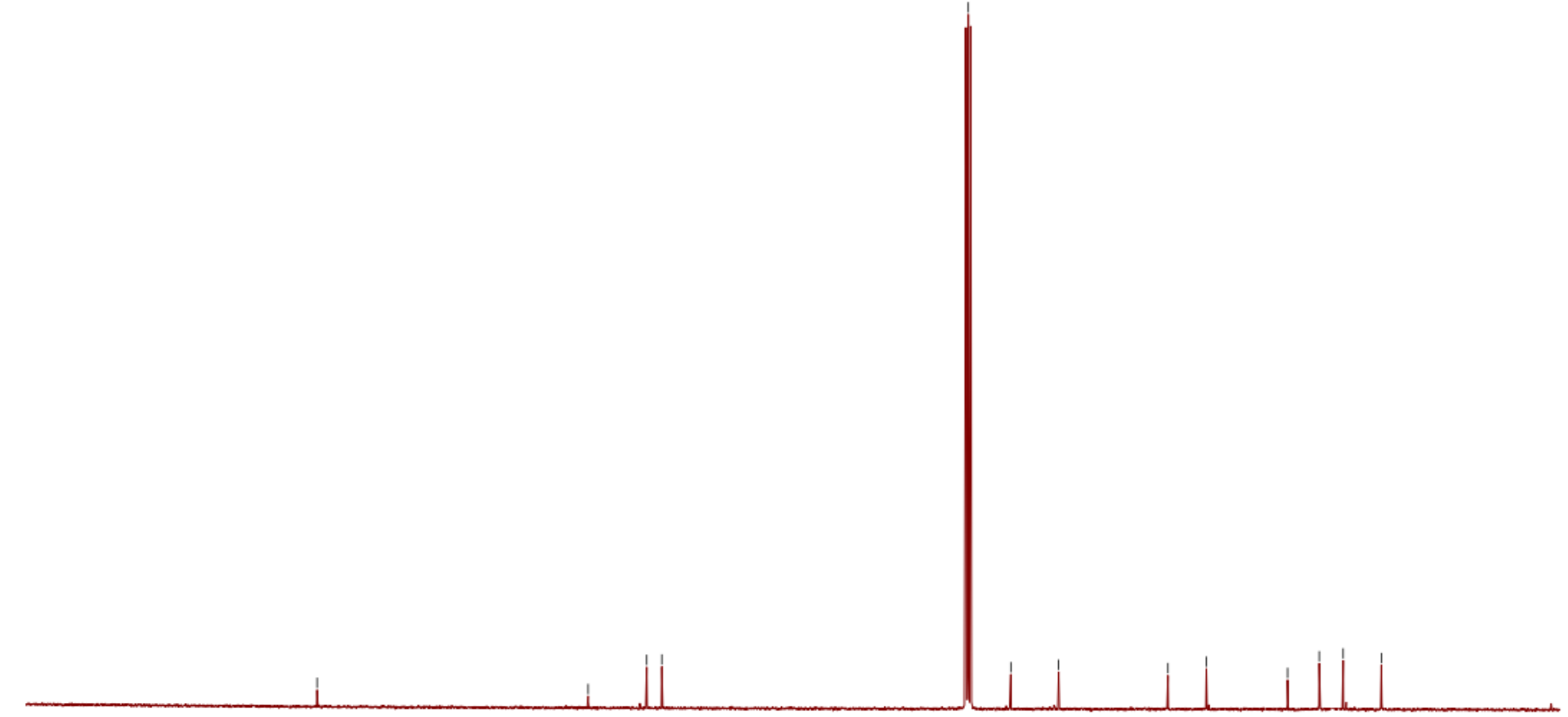

200

190

180

170

160

150

$140 \quad 130$

${ }_{120}^{1}$

$\begin{array}{ll}110 & 100 \\ & \delta(\mathrm{ppm})\end{array}$

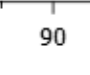

80

70

60

50

40

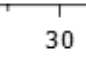

$20 \quad 10 \quad 0$ 
$\underline{\text { Selenophene boronate esters }}$

${ }^{1} \mathrm{H} 400 \mathrm{MHz}, \mathrm{CDCl}_{3}$
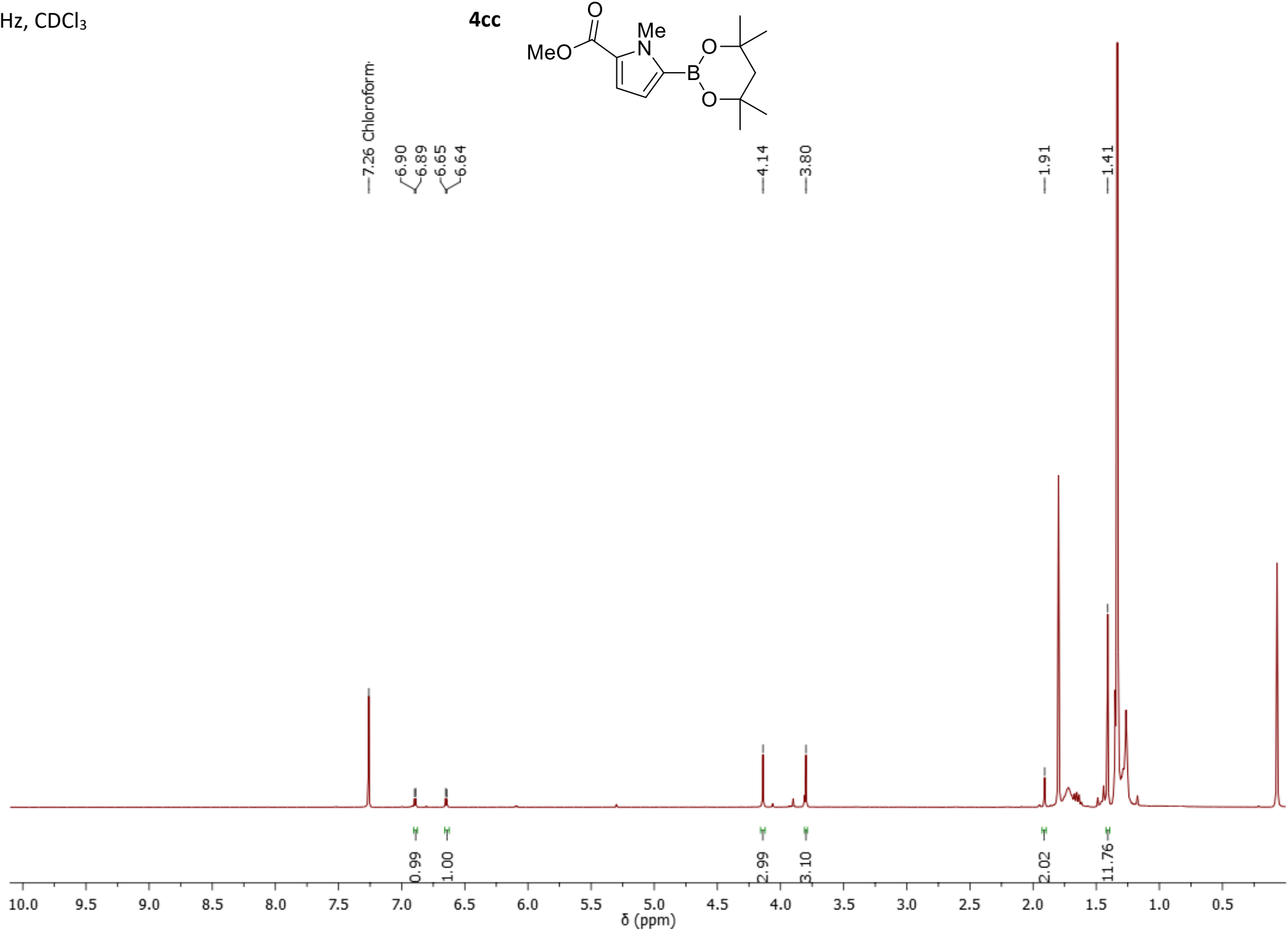
$\underline{\text { Selenophene boronate esters }}$

${ }^{13} \mathrm{C} 101 \mathrm{MHz}$

4cc
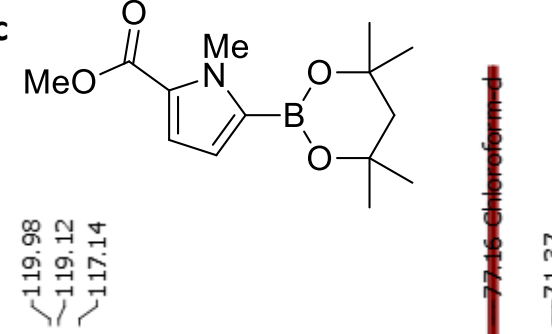

节产品事

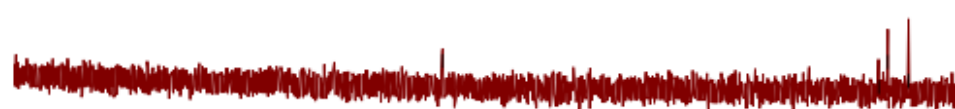


$\underline{\text { Selenophene boronate esters }}$

${ }^{1} \mathrm{H} 400 \mathrm{MHz}, \mathrm{CDCl}_{3}$
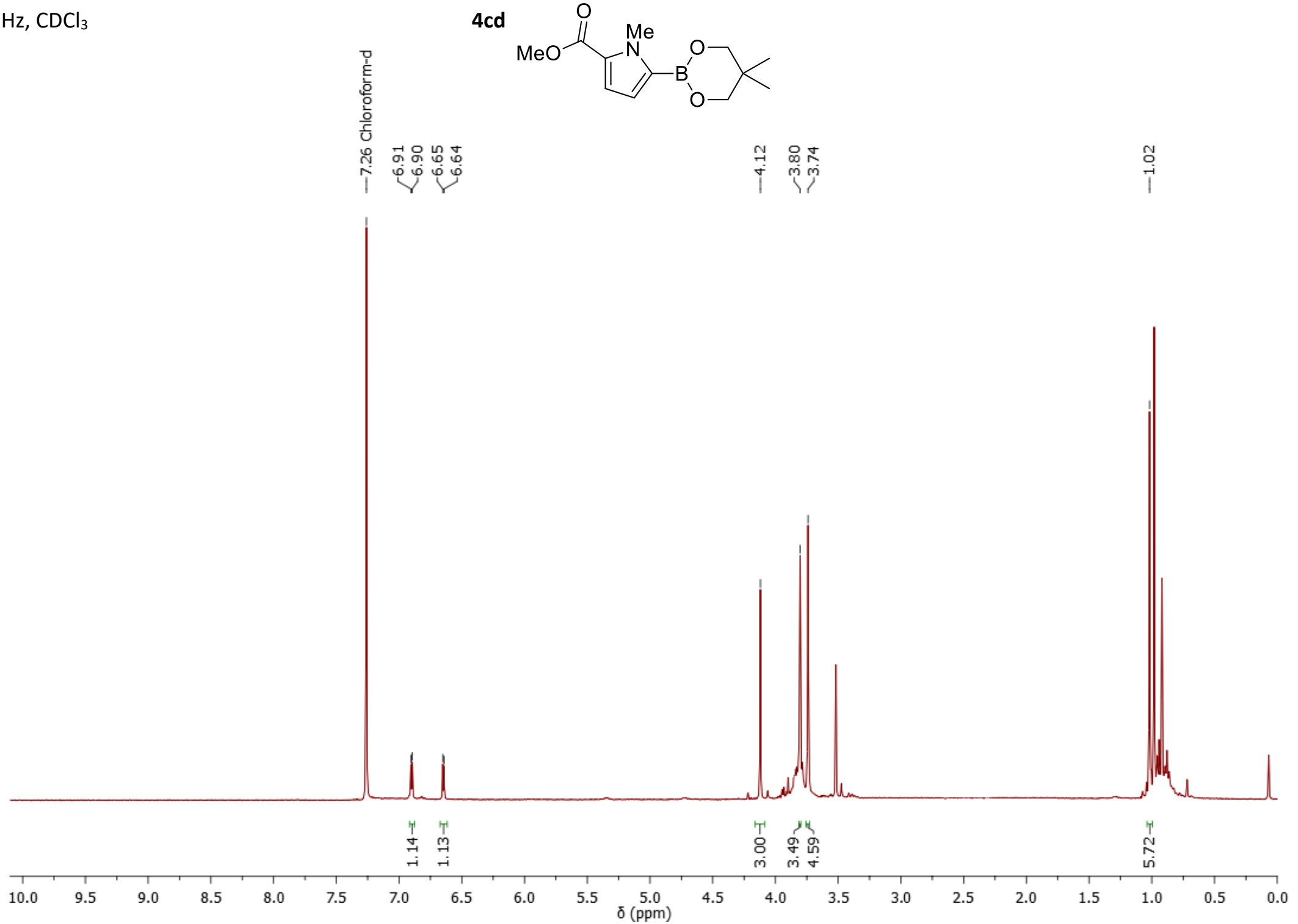
$\underline{\text { Selenophene boronate esters }}$

${ }^{13} \mathrm{C} 101 \mathrm{MHz}$

4cd

$$
\text { MeO }
$$$$
\text { n }
$$ 
$\underline{\text { Selenophene boronate esters }}$

${ }^{1} \mathrm{H} 400 \mathrm{MHz}, \mathrm{CDCl}_{3}$

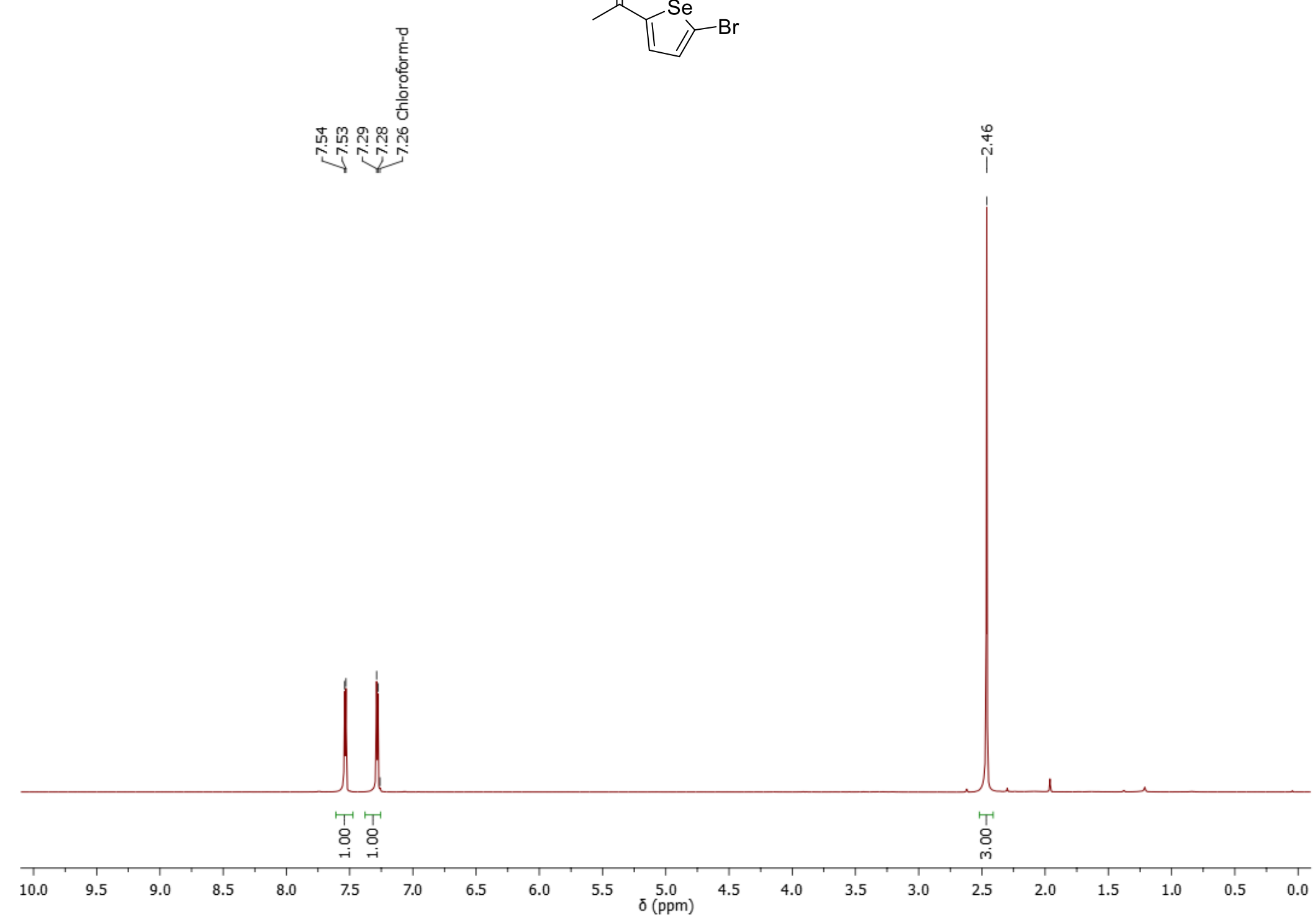


$\underline{\text { Selenophene boronate esters }}$

${ }^{13} \mathrm{C} 101 \mathrm{MHz}, \mathrm{CDCl}_{3}$

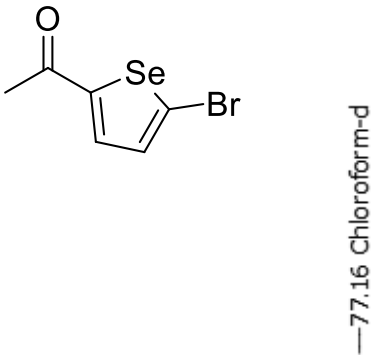

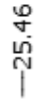

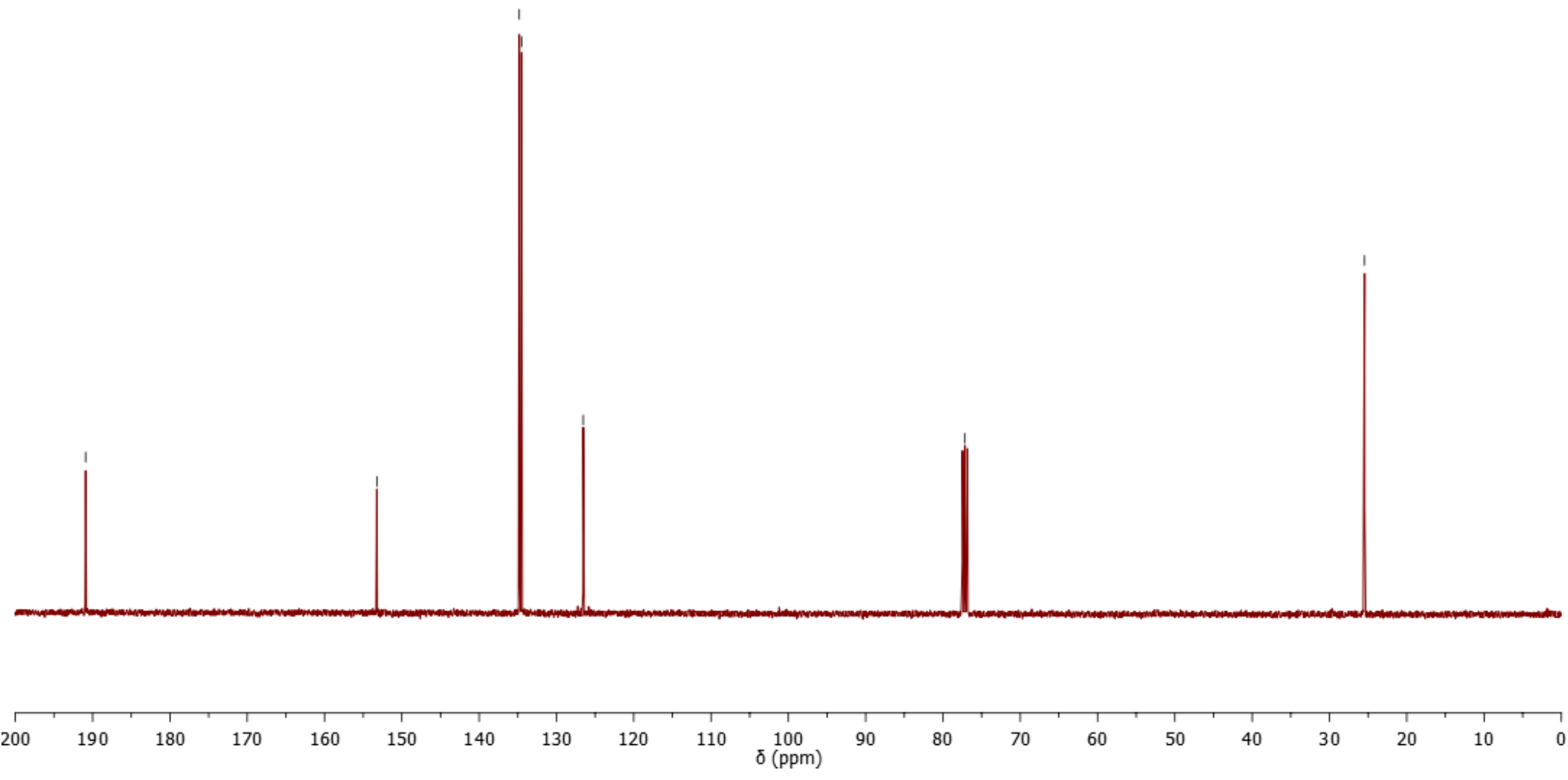


$\underline{\text { Selenophene boronate esters }}$

${ }^{1} \mathrm{H} 400 \mathrm{MHz}, \mathrm{CDCl}_{3}$

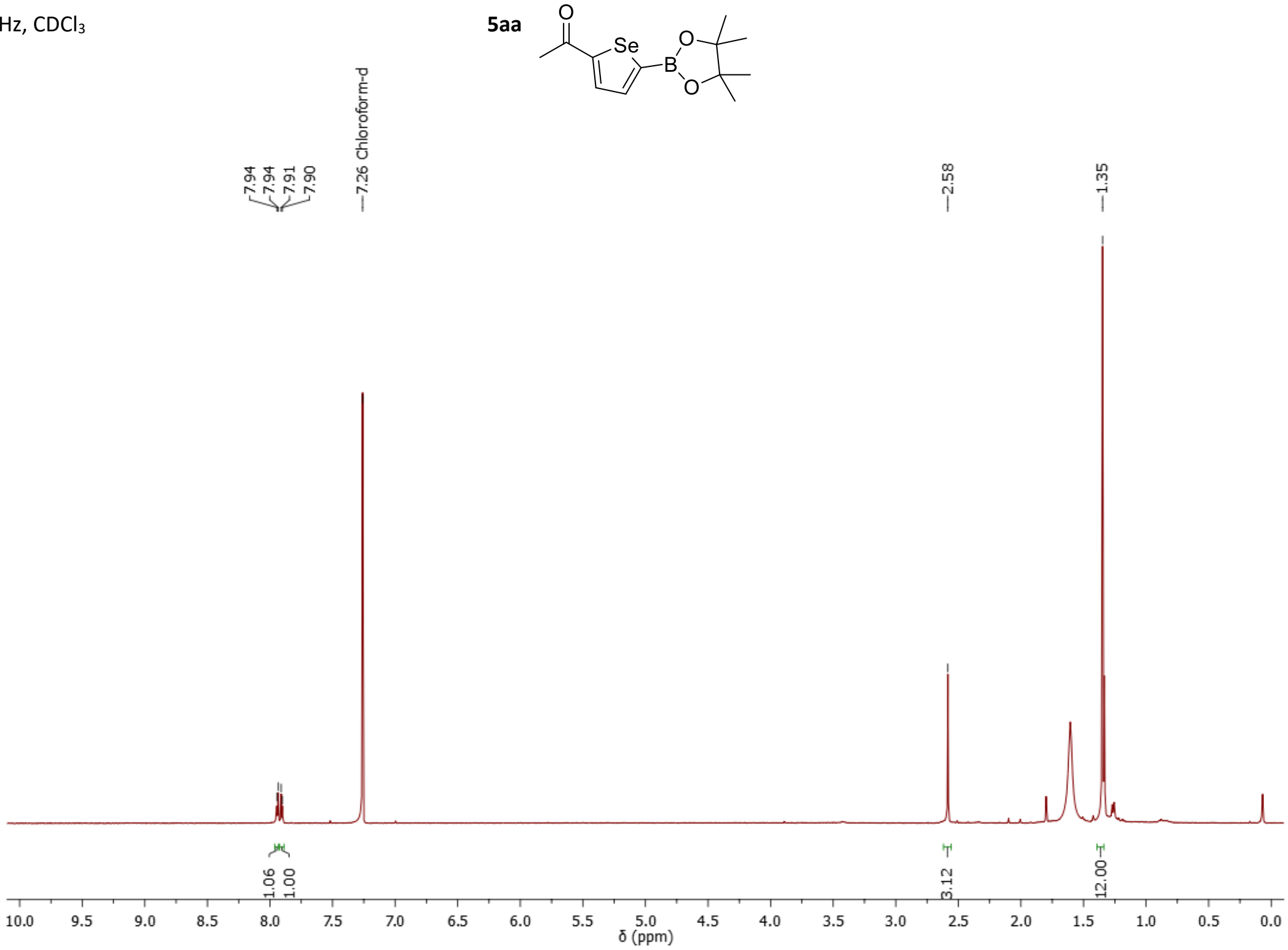

5 aa 
Selenophene boronate esters

${ }^{13} \mathrm{C} 101 \mathrm{MHz}, \mathrm{CDCl}_{3}$

$\stackrel{8}{N}$
N
$\stackrel{1}{1}$
$5 a a$

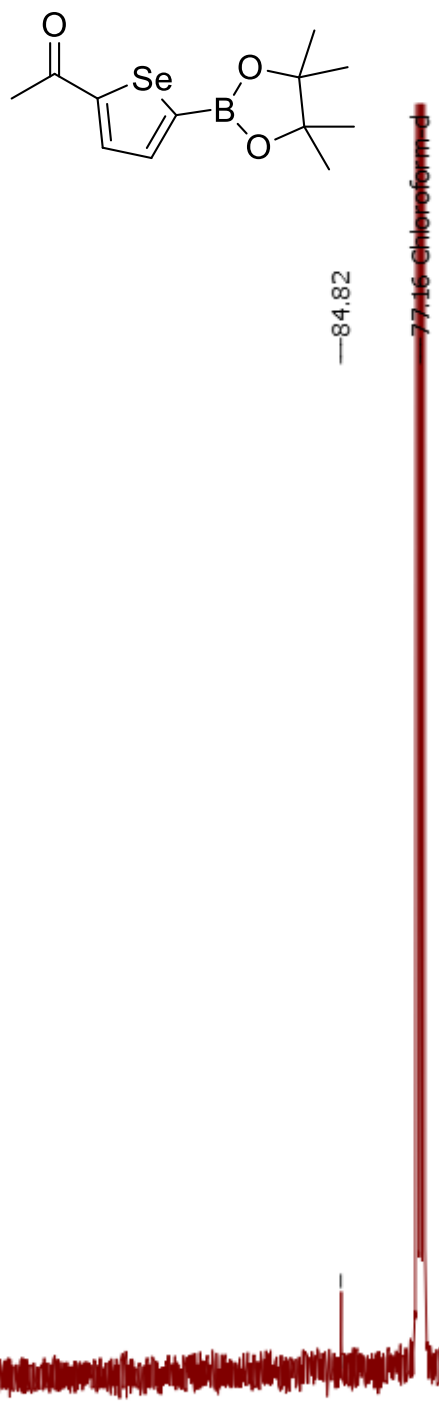

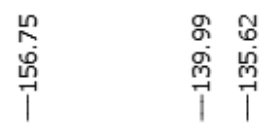

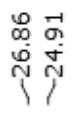

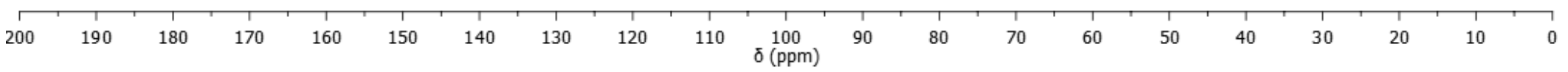

S154 
$\underline{\text { Selenophene boronate esters }}$

${ }^{1} \mathrm{H} 400 \mathrm{MHz}, \mathrm{CDCl}_{3}$

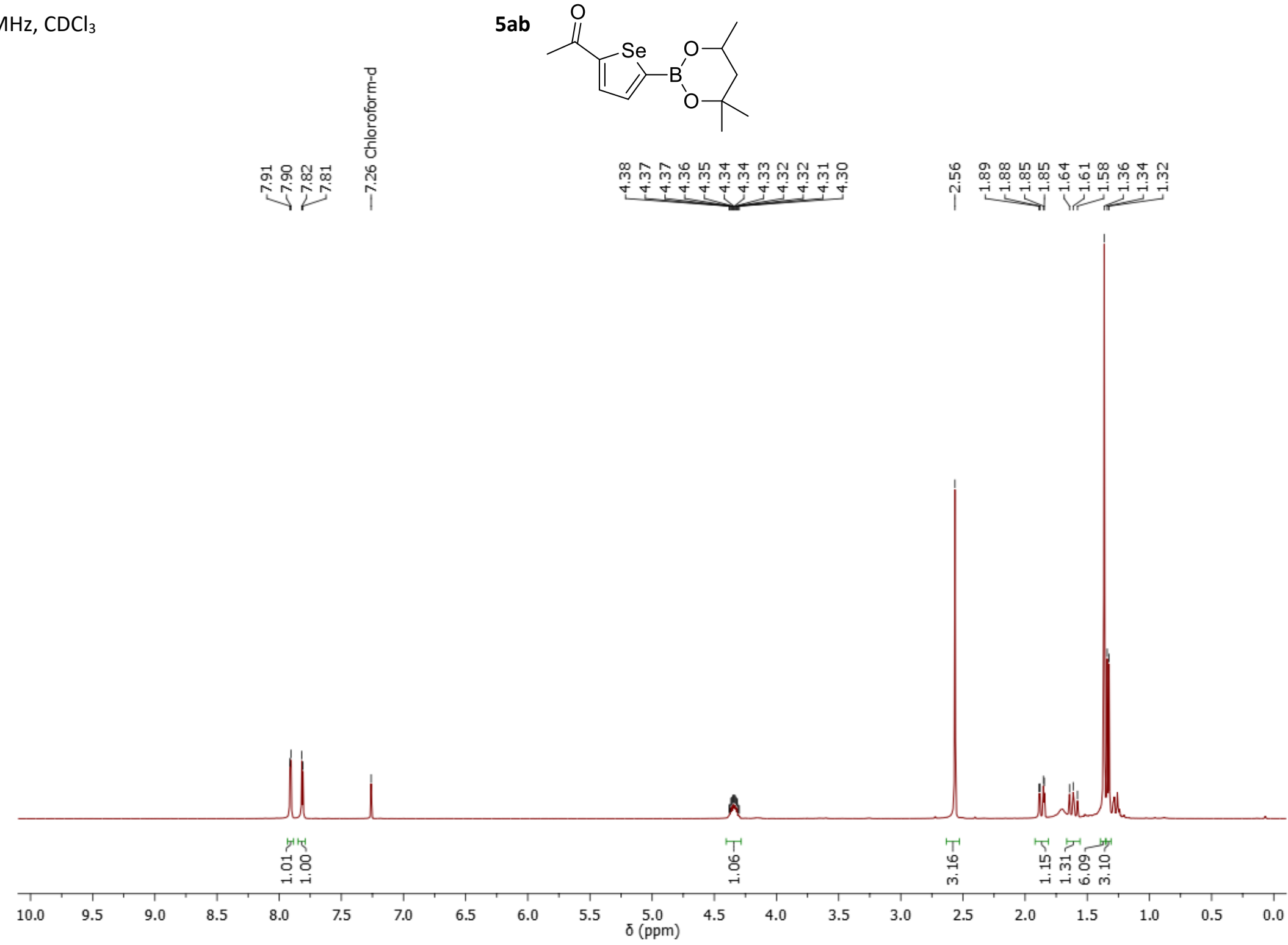

$5 a b$

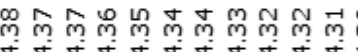


$\underline{\text { Selenophene boronate esters }}$

${ }^{13} \mathrm{C} 101 \mathrm{MHz}, \mathrm{CDCl}_{3}$

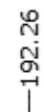

고ำ
$5 a b$

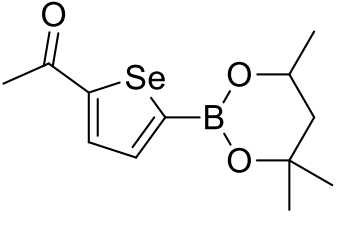

क्षे

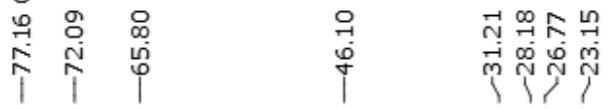

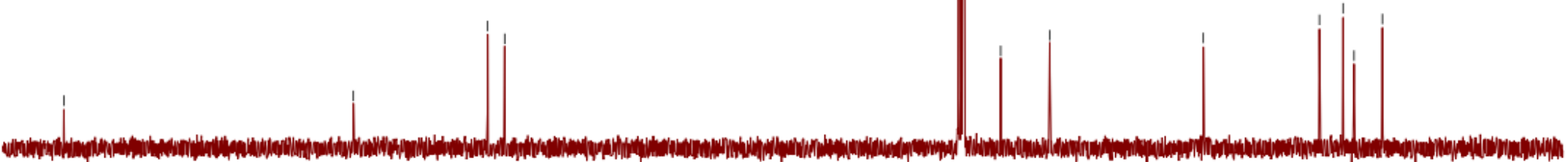

200 $190 \quad 180$ $170 \quad 160$ 150 140 130 120 100
$\delta(\mathrm{ppm})$ 80

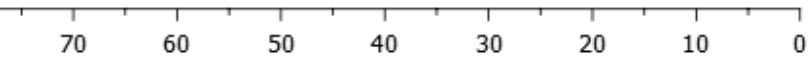


$\underline{\text { Selenophene boronate esters }}$

${ }^{1} \mathrm{H} 400 \mathrm{MHz}, \mathrm{CDCl}_{3}$

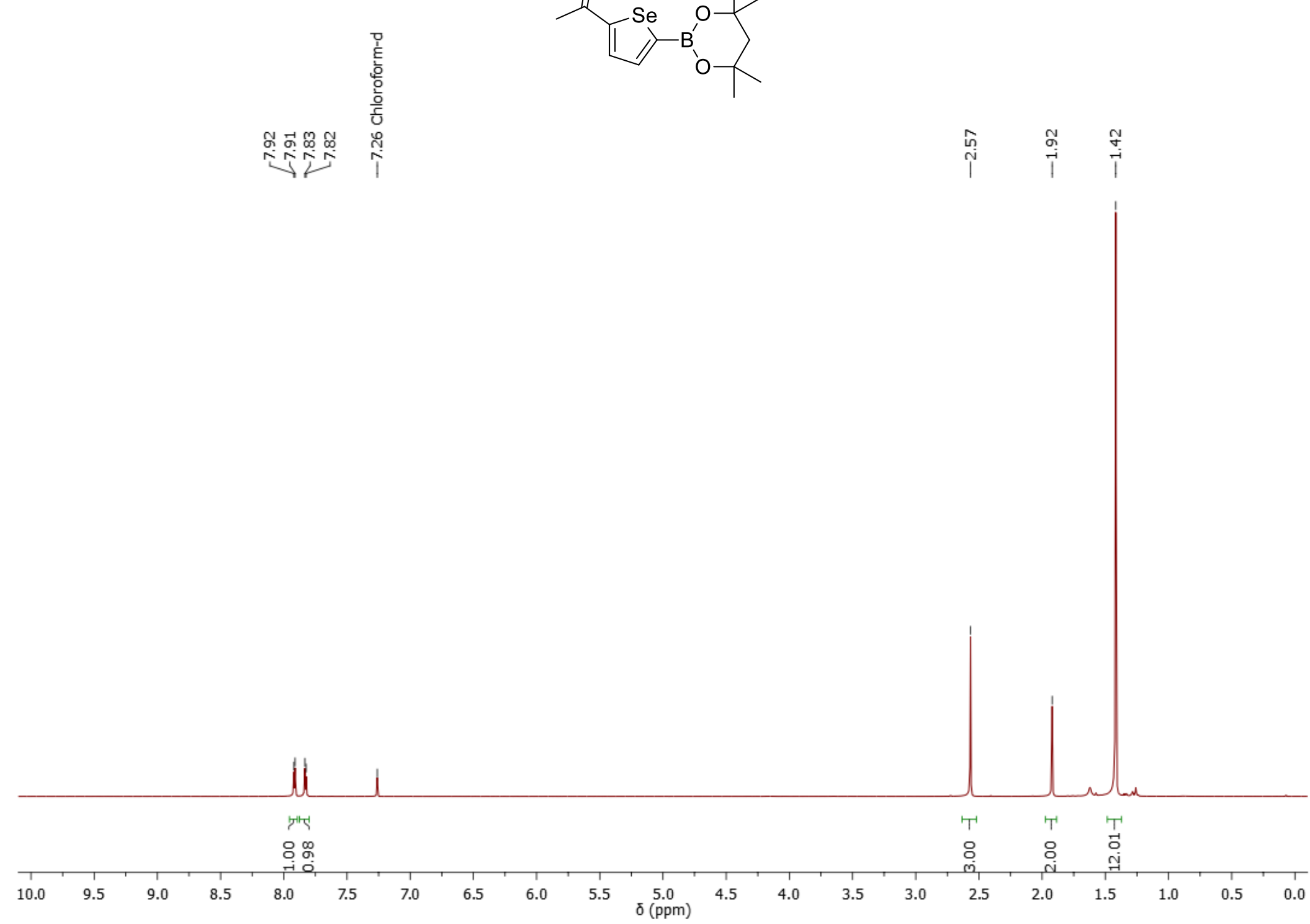


$\underline{\text { Selenophene boronate esters }}$

${ }^{13} \mathrm{C} 101 \mathrm{MHz}, \mathrm{CDCl}_{3}$

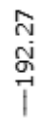

$5 a c$

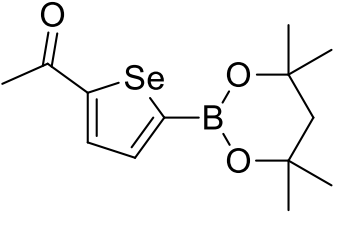

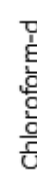

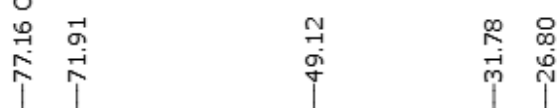

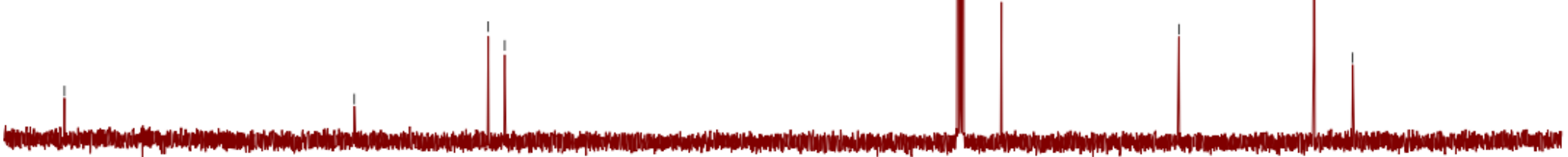

200 90 180 $170 \quad 160$ 150 $140 \quad 130$ 120 100
$\delta(\mathrm{ppm})$ 1
$90 \quad 80$ $\begin{array}{llllllll}70 & 60 & 50 & 40 & 30 & 20 & 10 & 0\end{array}$ 
$\underline{\text { Selenophene boronate esters }}$

${ }^{1} \mathrm{H} 400 \mathrm{MHz}, \mathrm{CDCl}_{3}$

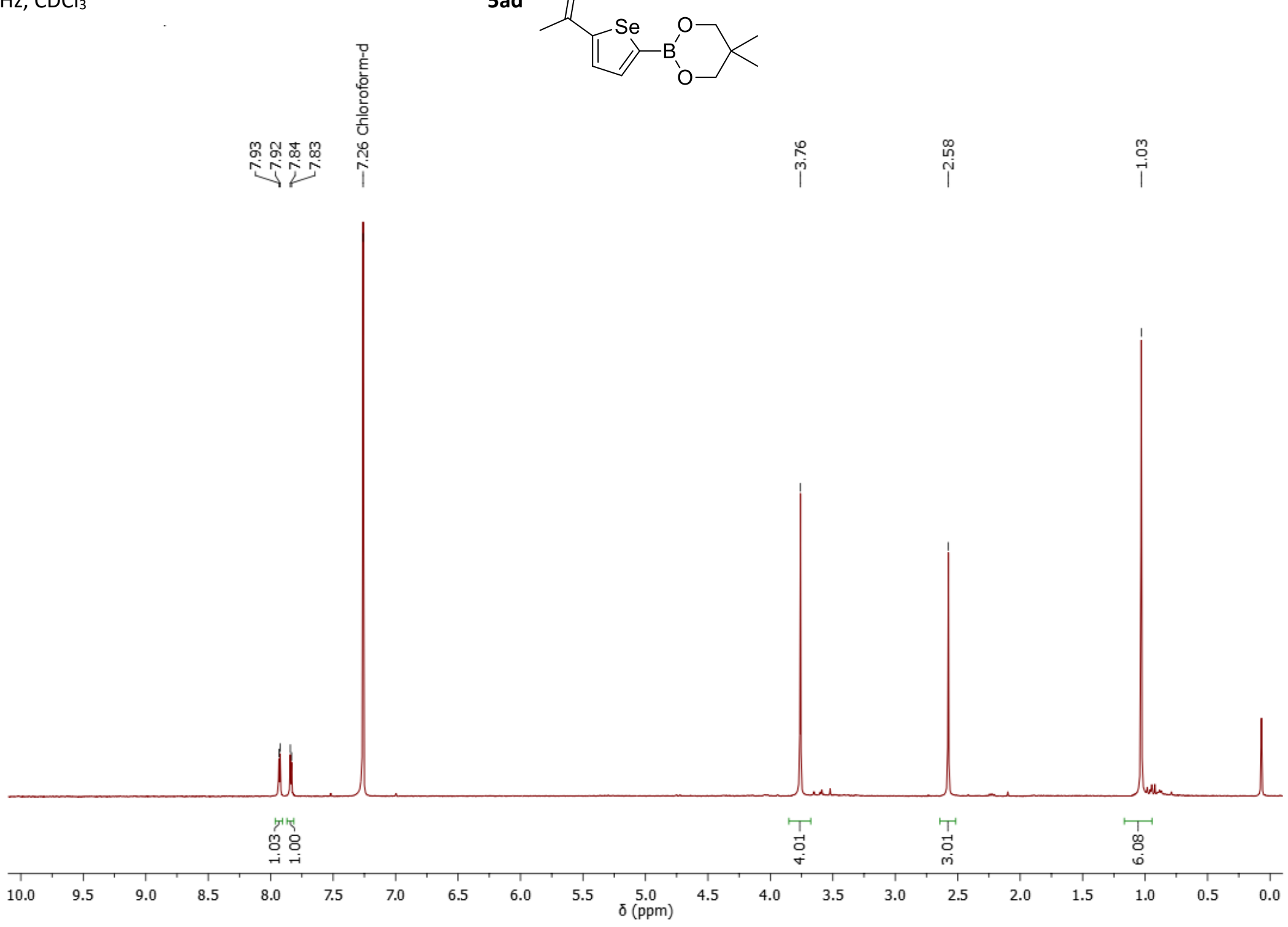


Selenophene boronate esters

${ }^{13} \mathrm{C} 101 \mathrm{MHz}, \mathrm{CDCl}_{3}$

5 ad

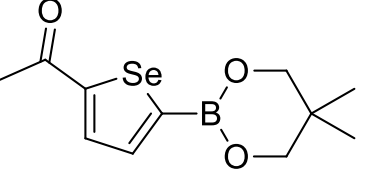

옹

능

离

일

mัN
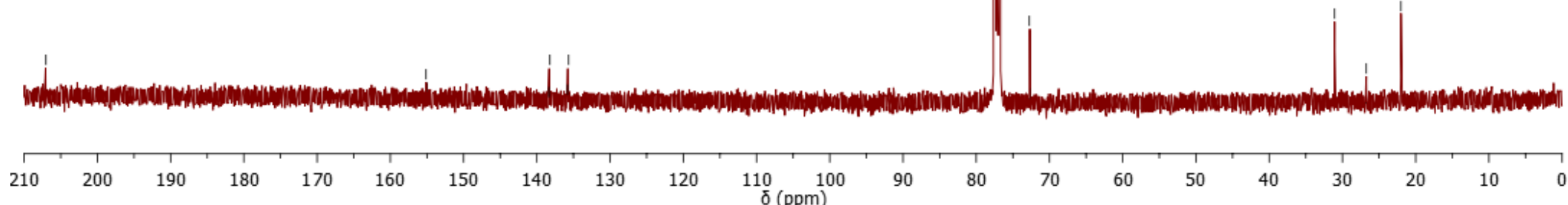


\section{Thiophene thioether}

${ }^{1} \mathrm{H} 400 \mathrm{MHz}, \mathrm{CDCl}_{3}$

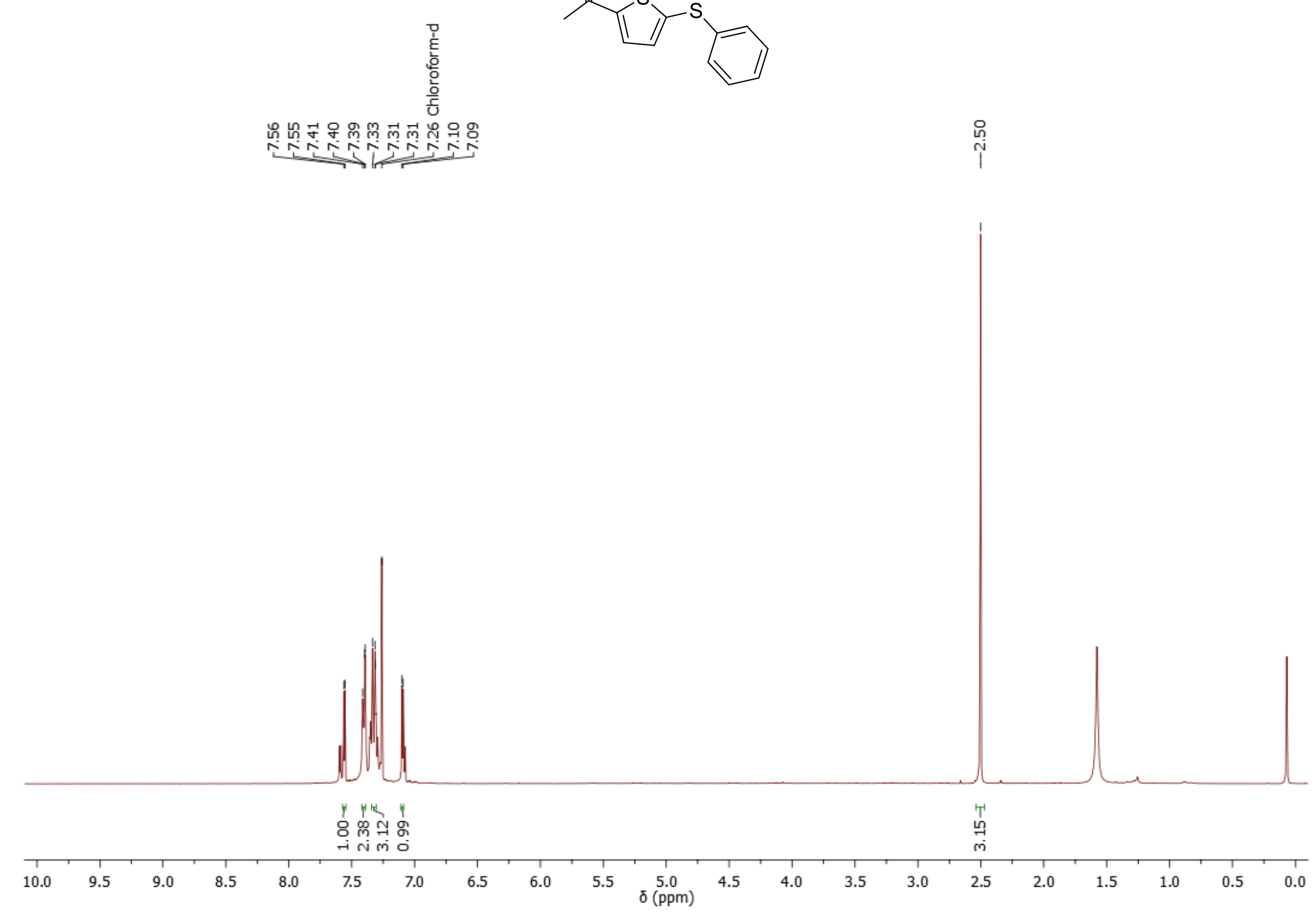




\section{Thiophene thioether}

${ }^{13} \mathrm{C} 101 \mathrm{MHz}$

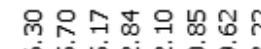

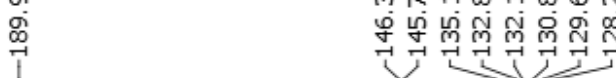

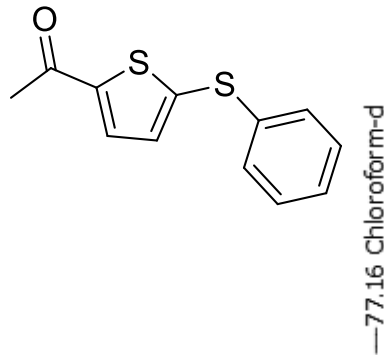

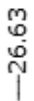

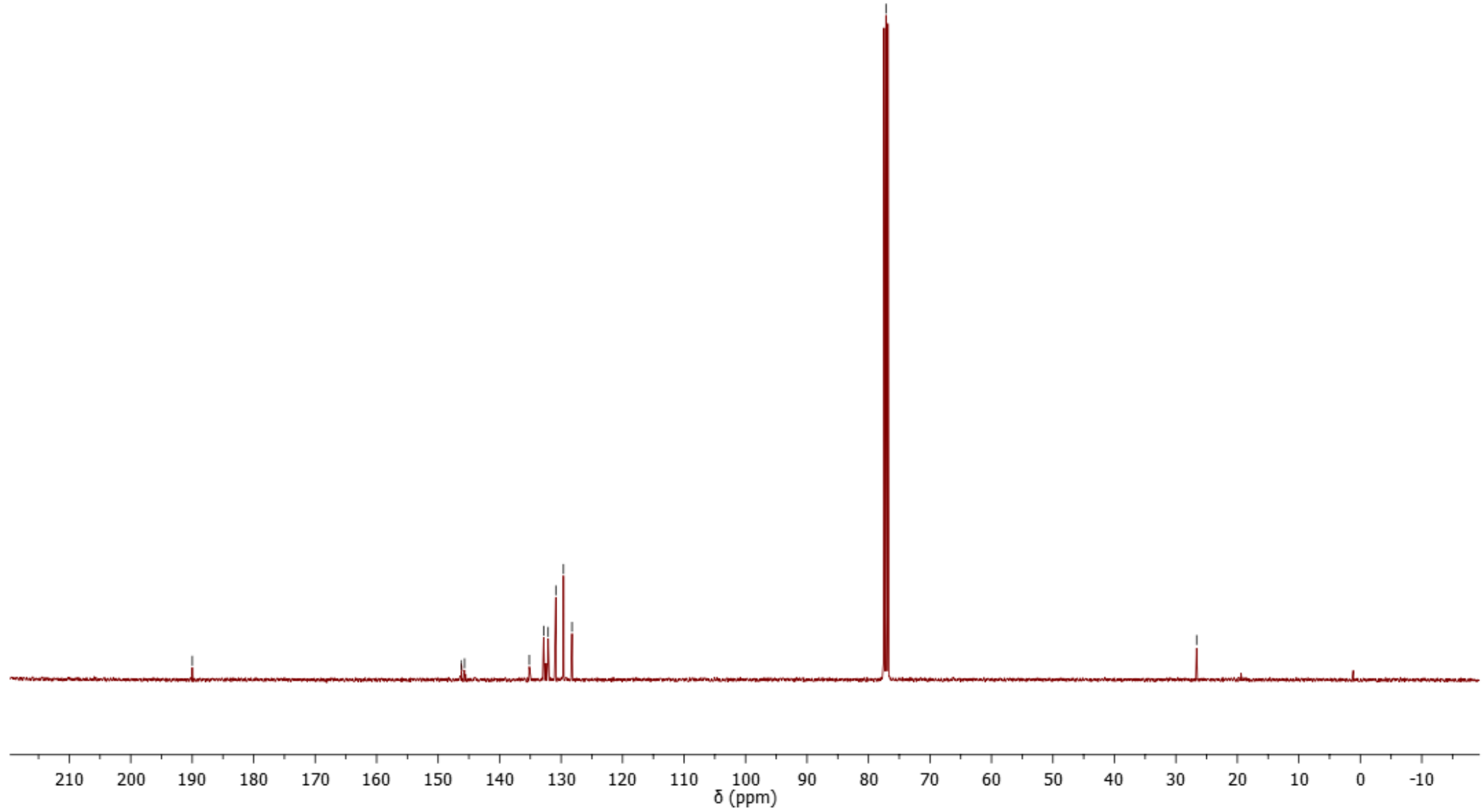




\section{Furan thioether}

${ }^{1} \mathrm{H} 400 \mathrm{MHz}, \mathrm{CDCl}_{3}$

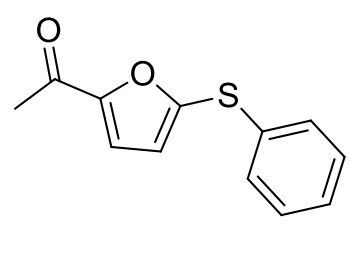

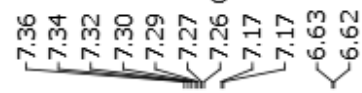

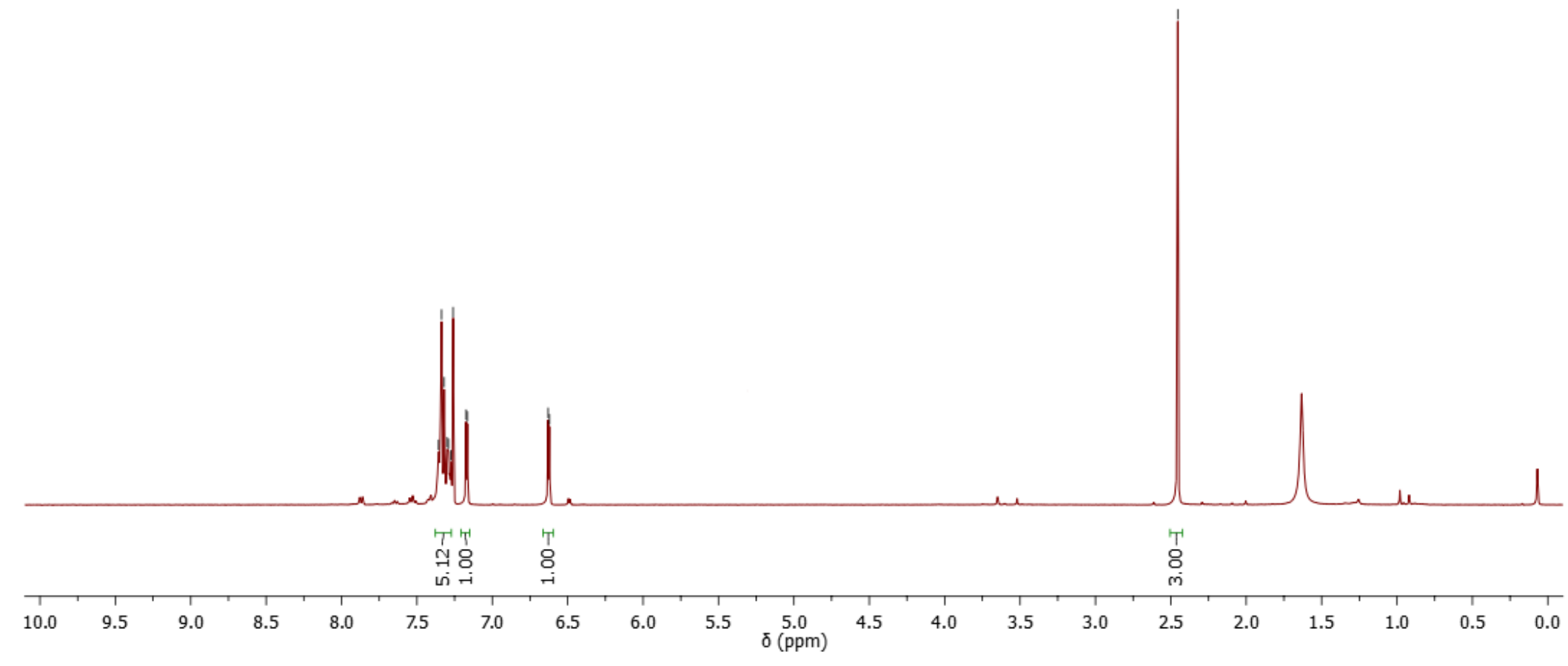




\section{Furan thioether}

${ }^{13} \mathrm{C} 101 \mathrm{MHz}$

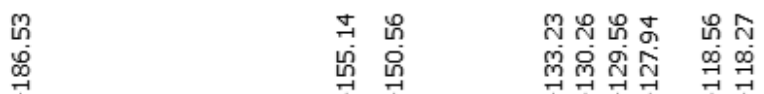



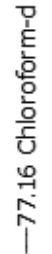

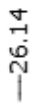

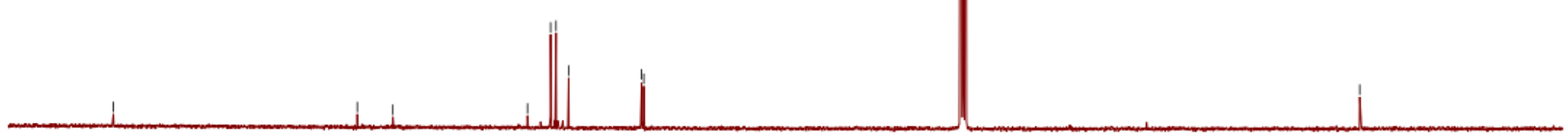

200

190

180

$170 \quad 160$

$150 \quad 140 \quad 130 \quad 120$

100
$\delta(\mathrm{ppm})$

$80 \quad 70$

1
60

$40 \quad 30$

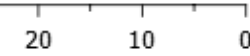

